\author{
Universidade de São Paulo \\ Instituto de Física
}

\title{
Teoria Inflacionária em Universos Anisotrópicos
}

\section{Thiago dos Santos Pereira}

Banca Examinadora:

Prof. Dr. Luis Raul Weber Abramo (IF/USP)

Prof. Dr. Victor de Oliveira Rivelles (IF/USP)

Prof. Dr. Fernando Tadeu Caldeira Brandt (IF/USP)

Prof. Dr. George Emanuel Avraam Matsas (IFT/UNESP)

Prof. Dr. Marcelo José Rebouças (CBPF) 



\section{Resumo}

Apresentamos neste trabalho uma generalização da teoria de perturbações cosmológicas para o caso de universos homogêneos e anisotrópicos, caracterizados por um espaço-tempo do tipo Bianchi I. Como aplicação da teoria, investigamos as conseqüências de uma fase inflacionária e anisotrópica do universo dos pontos de vista clássico e quântico. Após uma discussão da evolução do espaço-tempo de fundo nós quantizamos os modos perturbativos para, em seguida, construir o espectro de potências das perturbações de curvatura e de ondas gravitacionais do fim da inflação. Nossos resultados mostram que as principais características de uma fase anisotrópica primordial do universo são: (1) dependência direcional dos espectros de potências, (2) acoplamento entre as perturbações de curvatura e as ondas gravitacionais e (3) espectros distintos para as diferentes polarizações das ondas gravitacionais em grandes escalas cosmológicas. Todos esses efeitos são importantes apenas em grandes escalas cosmológicas e, localmente, recuperamos a teoria isotrópica de perturbações cosmológicas. Nossos resultados dependem de uma escala característica que pode, embora não seja estritamente necessário, ser ajustada a alguma escala observável. 



\begin{abstract}
In this work we generalize the standard theory of cosmological perturbations to the case of homogeneous and anisotropic universes described by a Bianchi I spacetime metric. As an application of this theory we investigate the predictions of an inflationary anisotropic phase, both at the classical and quantum level. After discussing the evolution of the background spacetime, we solve and quantize the perturbation equations in order to predict the power spectra of the curvature perturbations and gravity waves at the end of inflation. Our results show that the main features of an early anisotropic phase are: (1) a dependence of the spectra on the direction of the modes, (2) a coupling between curvature perturbations and gravity waves, and (3) the fact that the two gravity waves polarisations do not share the same spectrum on large scales. All these effects are significant only on large scales and die out on small scales where isotropy is recovered. Finally, our results depend on a characteristic scale that can, but a priori does not have to, be tuned to some observable scale.
\end{abstract}





\section{Estrutura da Tese}

A presente tese de doutorado consiste em um trabalho de generalização da teoria inflacionária isotrópica. Visando motivar essa generalização, dividimos a tese em duas partes. Na primeira delas apresentamos uma revisão da teoria isotrópica padrão, em seus aspectos exatos e linearmente perturbados. A segunda parte apresenta a generalização da teoria isotrópica, sendo que alguns resultados novos são obtidos para a teoria exata, e onde toda a teoria perturbativa é construída a partir de primeiros princípios. O leitor interessado apenas nos resultados originais dessa tese pode se ater, portanto, à segunda parte da mesma, bem como às referências [1, 2].

\section{Notações e Convenções}

Ao longo dessa tese usaremos unidades nas quais $c=\hbar=1$. A massa de Planck será definida como $M_{\mathrm{p}}=1 / \sqrt{8 \pi G}$ e a assinatura da métrica do espaço-tempo será $(-,+,+,+)$. Índices latinos representam coordenadas espaciais e índices gregos coordenadas espaço-temporais; índices repetidos subentendem soma. Pontos (". ") e linhas $("$, ") indicarão respectivamente derivadas com relação aos tempos físico $(t)$ e conforme $(\eta)$. Índice zero $(0)$ em quantidades físicas indica que as mesmas são calculadas no momento atual do universo. Todas essas convenções serão implicitamente adotadas, exceto em menção contrária. 



\section{Agradecimentos}

Em 2004, ano em que eu comecei meus estudos de doutoramento, meus interesses científicos apontavam para sub-áreas da cosmologia diferentes daquelas nas quais meu orientador, o Raul Abramo, consolidou-se como pesquisador. Tipicamente, a atitude do orientador nesse caso seria a de tentar redirecionar os interesses do aluno, ou mesmo a de sugerir um outro orientador para a continuidade do projeto. Felizmente, essas não foram as atitudes do meu orientador. Pelo contrário, o Raul viu nessa divergência de interesses uma manifestação, ainda que ingênua, da minha maturidade científica. Sua posição foi, desde o princípio, a de plena confiança e incentivo às minhas idéias. Nesse sentido, fui enormemente beneficiado pela sua experiência e, principalmente, pelo amplo conhecimento que o Raul tem dos vários aspectos da cosmologia moderna. No contexto científico atual, onde boa parte das pesquisas é conduzida por uma urgência má justificada por publicações, a atitude do Raul esteve acima de quaisquer vaidades e interesses pessoais, visando exclusivamente o desenvolvimento do meu conhecimento científico. É com muito respeito e admiração, portanto, que agradeço o Raul pela orientação e, sobretudo, pela amizade ao longo desses anos.

A realização deste trabalho não teria sido possível sem a participação do professor Jean-Philippe Uzan, a quem eu devo meus sinceros agradecimentos pela co-orientação, disposição e pela amizade. Também devo agradecimentos ao amigo Cyril Pitrou, o "Cirilo", pelas aulas de francês e pela amizade. A recepção calorosa que ele diz ter recebido no Brasil certamente não faz jus à que me foi oferecida na França. O "setor francês" dos agradecimentos deve ainda incluir o Institut d'Astrophysique de Paris (IAP), pela hospitalidade durante a realização deste trabalho.

Aos professores Sandro Fontanini e Luiz Roberto Evangelista, do Departamento de Física da Universidade Estadual de Maringá, pela minha formação como físico.

À Fapesp pelo apoio financeiro.

Às garotas da secretaria do Departamento de Física Matemática, Amélia e Simone, pela disponibilidade e pela paciência.

Aos amigos do Departamento de Física Matemática: Ivan Yasuda, Mário Baldiotti, João Assirati, Rodrigo Fresneda, Sérgio Jardino, Ronaldo Batista, Rafael Brandão, Carlos Molina e Milton Alexandre.

Aos amigos de Maringá e de sempre: Murilo de Cnop, Giovana Santos, Christian Fausto, Lígia Carreira e Junior Marcelo.

À minha família, pelo apoio e carinho.

À minha amiga, companheira, professora e esposa, Miriam Cristina, pelo carinho, pela paciência, por suportar minha ausência e, sobretudo, pelo amor incondicional... 

"What I am really interested in is whether God could have created the world in a different way..." Albert Einstein 

1 Modelo Cosmológico Padrão 15

1.1 Homogeneidade e Isotropia . . . . . . . . . . . . . . . . . . 15

1.2 Big Bang e a origem da $\mathrm{RCF} \ldots \ldots \ldots \ldots \ldots \ldots \ldots \ldots$

1.3 Alguns problemas . . . . . . . . . . . . . . 24

2 Inflação 28

2.1 Resolvendo os problemas do Big Bang . . . . . . . . . . . 29

2.2 O Inflaton . . . . . . . . . . . . . . . . . . . 32

2.3 Quantização de um modelo protótipo . . . . . . . . . . . . 38

3 Teoria Isotrópica de Pertubações Cosmológicas 42

3.1 Perturbações Lineares . . . . . . . . . . . . . . . . . . . . . . 42

3.2 Decomposição EVT . . . . . . . . . . . . . . . . . . . . 44

3.3 Invariância de Calibre . . . . . . . . . . . . . . 46

3.4 Equações Linearizadas . . . . . . . . . . . . . . . . . 50

4 Quantização das Perturbações $\quad 54$

4.1 Formalismo ADM . . . . . . . . . . . . . . . . 55

4.2 Procedimento Geral de Quantização . . . . . . . . . . . . . . . 57

4.3 Conclusões . . . . . . . . . . . . . . . . . . . . . . 62

\section{Teoria Anisotrópica $\quad 64$}

5 Dinâmica Homogênea 69

5.1 Forma geral da métrica . . . . . . . . . . . . . . . 69

5.2 Equações de Friedmann e Soluções Gerais . . . . . . . . . . . 70

5.3 Regime de Rolagem Lenta . . . . . . . . . . . . . . . . . . 76

5.4 Inflação Caótica: integração numérica . . . . . . . . . . . . . 77

5.5 Discussão . . . . . . . . . . . . . . . . . . . 82 
6 Dinâmica Perturbativa $\quad \mathbf{8 3}$

6.1 Decomposição dos modos perturbativos . . . . . . . . . . . . . 83

6.2 Variáveis invariantes de calibre . . . . . . . . . . . . . 87

6.3 Dinâmica das perturbações . . . . . . . . . . . . . . . . . . 90

6.4 Equações reduzidas e variáveis de Mukhanov-Sasaki . . . . . . . . . . . . . . . . . 94

6.5 Limite de pequenos comprimentos de onda . . . . . . . . . . . . . . 97

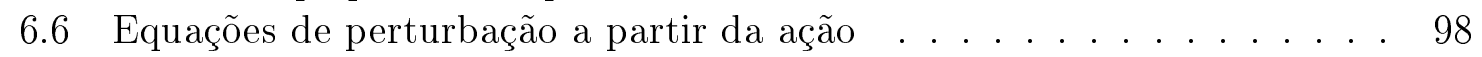

7 Quantização das Perturbações e Previsões $\quad 102$

7.1 Universos de FRW . . . . . . . . . . . . . . . . . . . . 102

7.2 Universos de Bianchi I . . . . . . . . . . . . . . 106

7.3 Prescrição para as condições iniciais . . . . . . . . . . . . . . . . 112

7.4 Liberdade da parametrização temporal . . . . . . . . . . . . . . . . . . . . . . . . . . . . . . . . . . . . . .

7.5 Discussão . . . . . . . . . . . . . . . . . . . 116

7.6 Espectros das Perturbações Primordiais . . . . . . . . . . . . . 117

7.7 Previsões . . . . . . . . . . . . . . . . . . . . 121

8 Conclusões Finais e Perspectivas $\quad 124$

$\begin{array}{ll}\text { Referências Bibliográficas } & 127\end{array}$

A Fórmulas Gerais

A.1 Funções de Hankel . . . . . . . . . . . . . . . . . . . . . . . . 132

A.2 Decomposição das ondas planas . . . . . . . . . . . . . . 132

B Detalhes dos Cálculos Algébricos 133

B.1 Equações anisotrópicas de fundo . . . . . . . . . . . . . . . . . . . 133

B.2 Propriedades da decomposição EVT . . . . . . . . . . . . . . . . . . . . . . . . . . . . . . . . 134

B.3 Detalhes dos cálculos perturbativos . . . . . . . . . . . 135

B.4 Derivada total na ação . . . . . . . . . . . . . . . . 136

C Detalhes dos Cálculos Numéricos $\quad 137$

C.1 Evolução dos componentes do tensor de cisalhamento . . . . . . . . . . . 137

C.2 Aproximação de Rolagem Lenta . . . . . . . . . . . . . . . . . . . . . . . 140

C.3 Caso Particular $\alpha=\pi / 2 \ldots \ldots \ldots \ldots$. . . . . . . . . . . . . . . . . . . . . . 


\section{Parte I}

\section{Teoria Isotrópica}





\section{Introdução}

A concepção atual de cosmologia como ramo de pesquisa científica difere drasticamente do que se acreditava ser a "ciência do universo" cem anos atrás. Essa transição conceitual fica evidente ao percorrermos a história da cosmologia ao longo do século XX. Nesses cem anos foram vários os acontecimentos que garantiram definitivamente à cosmologia o status de ciência física. Do lado teórico, os trabalhos seminais de Albert Einstein, particularmente a sua teoria da relatividade geral, estabeleceram as bases matemáticas da cosmologia moderna. Do lado observacional, a descoberta da expansão do universo pelo astrônomo Edwin Hubble em 1929 [3] e a detecção da Radiação Cósmica de Fundo (RCF) por Arnold A. Penzias e Robert W. Wilson em 1965 [4] apontavam para uma mudança drástica de paradigma onde o universo estático e finito de Einstein era substituído por uma estrutura dinâmica e muito mais rica do que até então se imaginava. Estabeleciam-se assim as bases conceituais da cosmologia moderna. Foi contudo no final da década de 80 que - com o lançamento do satélite COBE [5] - a chamada era da Cosmologia de Precisão teve início, culminando com a concessão do Prêmio Nobel para os físicos John C. Mather e George F. Smoot. Desde então o crescimento vertiginoso das pesquisas teóricas e observacionais em cosmologia permitiu que os cosmólogos substituíssem a prudência dos argumentos especulativos em favor da prática do rigor científico.

Atualmente, a atividade de pesquisa em cosmologia é intensa. Os cinco primeiros anos de atuação do satélite WMAP $[6,7,8]$ foram decisivos para o estabelecimento do que hoje chamamos de modelo cosmológico padrão, ou seja, um universo homogêneo e isotrópico com cerca de 13,9 bilhões de anos, composto por $73 \%$ de energia escura, $23 \%$ de matéria escura, $4 \%$ de matéria comum (bariônica) e apenas $0.01 \%$ de radiação eletromagnética [9]. Não apenas as propriedades globais do universo, o modelo padrão contempla também as estruturas de grandes escalas tais como galáxias e seus aglomerados. Nesse contexto a teoria do universo primordial, ou teoria cosmológica inflacionária, desempenha um papel central.

Inicialmente formulada para resolver alguns problemas do modelo quente do universo (o Big Bang) [10], a teoria inflacionária tornou-se responsável por conciliar num mesmo arcabouço teórico as hipóteses de homogeneidade e isotropia do universo com as observações das estruturas em grandes escalas. A importância dessa característica da teoria fica evidenciada pelo seguinte (aparente) paradoxo: como é possível que, a 
partir de uma distribuição perfeitamente densa e homogênea de partículas - tal como prevista pelo modelo do Big Bang - possam surgir as estruturas astrofísicas que observamos? A resposta da teoria inflacionária a essa pergunta é a de que a existência de um período primordial de expansão acelerada, ou inflação, poderia varrer para fora do horizonte causal toda flutuação presente nos campos quânticos primordiais. Uma vez fora do horizonte causal essas flutuações permanecem "congeladas" até o instante em que a conseqüente evolução do universo, volta a englobá-las em seu domínio de causalidade. A partir daí essas flutuações primordiais atuam como as condições iniciais de um processo de instabilidade gravitacional do qual se originam as estruturas de grandes escalas que conhecemos, bem como as flutuações de temperatura da RCF. Como conseqüências observacionais, o modelo prevê que as distribuições de energia e temperatura do universo sejam de caráter gaussiano - em acordo com o teorema do limite central - e que estejam distribuídas de forma aproximadamente independente de escalas. Tais previsões têm sido sistematicamente confirmadas pelas medições das flutuações de temperatura da RCF. Em particular os dados do quinto ano de atuação do satélite WMAP confirmam que tais flutuações são gaussianas e com uma dependência de escala da ordem de $4 \%$ [9].

No que diz respeito à redução do espaço de parâmetros do modelo de concordância cósmica, portanto, a situação atual é extremamente satisfatória. O foco das pesquisas se direciona agora na compreensão de anomalias que, evidenciadas pelos dados atuais, não se enquadram no modelo cosmológico padrão. Especialmente anômalos são os dados das flutuações de temperatura das grandes escalas cosmológicas; são as chamadas anomalias de baixos multipolos da RCF. Dentre as mais graves destacamos o baixo valor das correlações em grandes escalas angulares, especialmente do quadrupolo $C_{2}[6,7,11,12,13]$, o alinhamento entre o quadrupolo e o octopolo [11, 14, 15, 16], a planaridade do octopolo [13] e a alta esfericidade do termo de hexadecupolo [16]. Todas essas anomalias são extremamente improváveis do ponto de vista teórico e, contudo, observadas com diferentes graus de confiança estatística, variando do razoável ao altamente significativo.

As explicações teóricas para esses fenômenos são as mais diversas possíveis, passando por deficiências intrínsecas de construtos estatísticos a posteriori [17], contaminações de origem galácticas e astrofísicas [16, 18, 19], campos magnéticos primordiais [20], topologias exóticas do universo [21, 22] e modelos do universo jovem [1, 2, 23, 24]. Todas têm em comum a crença generalizada de que as anomalias sejam uma manifestação de possíveis falhas das hipóteses subjacentes ao modelo padrão, especialmente no que diz respeito às hipóteses de isotropia e gaussianidade do universo. Se a origem das anomalias observadas for decorrente de aspectos puramente não-gaussianos do universo, então a abordagem teórica do problema se torna bastante difícil, uma vez que a possibilidade de modelos compatíveis com observáveis não-gaussianos é virtualmente infinita. Além disso, sinais primordiais de não-gaussianidade são fortemente suprimidos pela expansão do universo [25, 26], o que torna sua detecção na radiação cósmica de fundo praticamente impossível frente à precisão das medidas atuais e futuras. Não obstante, a busca por desvios de gaussianidade na RCF é tema de intensa pesquisa [27].

Uma outra explicação para as anomalias de grandes escalas da RCF está na falha do princípio cosmológico, que postula a homogeneidade e isotropia do universo 
em grandes escalas. Em outras palavras, as equações que governam os observáveis cosmológicos são invariantes por rotações e translações. Esse princípio é de extrema importância e forma, juntamente com as equações de Einstein, a base do modelo padrão. Sua implementação é amplamente justificada pelo espectro de temperatura da RCF e pela distribuições de galáxias observadas [28]. Sua validade, no entanto, pode ser questionada em segunda ordem, já que a existência de uma direção ou posição privilegiada poderia se manifestar na distribuição de matéria e energia do universo.

É dentro desse contexto que o presente trabalho pretende contribuir para a compreensão do problema. Precisamente, nos propomos a abandonar a hipótese de isotropia do universo e assim reformular a teoria das perturbações cosmológicas sobre um espaço-tempo anisotrópico do tipo Bianchi I. São duas nossas principais motivações. Em primeiro lugar, a descrição de uma fase inflacionária sobre espaços-tempo anisotrópicos é totalmente compatível com as motivações iniciais que levaram à idéia da inflação. De fato, um dos sucessos da teoria inflacionária é justamente o de tornar um universo arbitrariamente inomogêneo e anisotrópico na estrutura que hoje observamos. Dessa forma a reformulação da teoria em espaços-tempo anisotrópicos não apenas põe à prova a robustez da hipótese inflacionária como revela estruturas inexistentes no modelo isotrópico padrão. Em segundo lugar, as principais conseqüências de uma quebra de isotropia do universo jovem se manifestam, como veremos, nas grandes escalas cosmológicas. Isso significa que o impacto de um período anisotrópico primordial deve se verificar justamente nas escalas onde se observam as anomalias da RCF. Embora não tenhamos comparado nosso modelo diretamente aos dados observacionais nesse trabalho, veremos que mesmo as previsões teóricas mais simples da fase anisotrópica têm de fato algo a dizer sobre as anomalias da RCF.

Começaremos o capítulo 1 com uma revisão da cinemática e da dinâmica de um universo homogêneo e isotrópico, dando ênfase aos aspectos importantes para o modelo inflacionário. Apresentaremos nesse mesmo capítulo (§1.2) uma breve revisão da história térmica do universo e dos problemas do modelo do Big Bang que levaram à idéia da inflação cosmológica. Essa idéia, por sua vez, será introduzida no capítulo 2 e, em $\S 2.2$, a implementaremos através de um modelo com um campo escalar. Faremos no capítulo 3 uma exposição matemática da teoria isotrópica das perturbações cosmológicas que nos levará, em §3.4, às equações dinâmicas dos modos perturbativos durante a era inflacionária. Essas perturbações serão quantizadas no capítulo 4 e os principais observáveis cosmológicos serão construídos em $\S 4.2$. Concluiremos a primeira parte dessa tese com uma discussão sobre as principais características do modelo inflacionário padrão. 


\section{CAPÍTULO 1}

\section{Modelo Cosmológico Padrão}

É natural que a cosmologia, mais do que outras ciências físicas, abuse de artifícios indiretos tais como extrapolações e hipóteses. A razão disso é muito simples: seu objeto de estudo - o universo - é infinitamente maior que o alcance espaço-temporal dos nossos instrumentos de medida. Em particular, a construção do modelo cosmológico padrão depende de uma hipótese e de uma extrapolação fundamentais. A hipótese é a de que o universo seja, em grandes escalas, homogêneo e isotrópico (princípio cosmológico). A extrapolação é a de que a teoria da relatividade geral, cuja validade em escalas astrofísicas tem sido consistentemente confirmada por diferentes experimentos, seja também válida em escalas cosmológicas. A importância desses dois ingredientes se justifica a posteriori pela concordância do modelo com os dados observacionais.

\subsection{Homogeneidade e Isotropia}

Observações cuidadosas do céu mostram que a distribuição de galáxias ao nosso redor é, com boa aproximação, isotrópica. Essa isotropia é ainda mais marcante na distribuição angular de temperatura da RCF, que apresenta flutuações de uma parte em $10^{5}$ [8, 9]. Poderíamos portanto supor que o universo é simétrico em torno de um ponto especial: a Terra. Contudo, a história da ciência nos ensina que a Terra não ocupa um lugar privilegiado no espaço. Essa é a síntese do princípio copernicano. Sua validade é justificada pela observação da distribuição espacial de galáxias. A análise do catálogo de galáxias disponibilizado pela equipe do Sloan Digital Sky survey [29], por exemplo, mostra que a distribuição de galáxias é homogênea em escalas maiores que 300 milhões de anos luz [28]. Portanto, a isotropia que observamos deve ser também observada em qualquer outra galáxia. Isso implica que a geometria espacial do universo num dado instante deva ser descrita por uma métrica que é globalmente homogênea e isotrópica. Como se sabe, espaços com esse grau de simetria são representados pelo seguinte tensor (tridimensional) de Riemann [30]

$$
{ }^{(3)} R_{i j k l}=K\left(g_{k j} g_{i l}-g_{l j} g_{i k}\right)
$$


onde $g_{i j}$ é a métrica espacial e $K \in\{-1,0,1\}$ é a curvatura da seção espacial. Como veremos, a curvatura está relacionada à quantidade de energia do universo e, portanto, seu valor real deve ser determinado pela natureza ${ }^{1}$.

Obviamente, um observador arbitrariamente acelerado em direção ao centro da galáxia verá uma distribuição anisotrópica e irregular de matéria e temperatura. Em outras palavras, a expansão do universo privilegia uma classe de observadores para os quais o mesmo é homogêneo e isotrópico, são os chamados observadores comóveis. Para esses observadores, a métrica do universo define o elemento de linha de FriedmannRobertson-Walker (FRW)

$$
\mathrm{d} s^{2}=-\mathrm{d} t^{2}+a^{2}(t)\left[\frac{\mathrm{d} r^{2}}{1-K r^{2}}+r^{2} \mathrm{~d} \Omega^{2}\right],
$$

onde a função real a(t) é o fator de escala do universo e $\mathrm{d} \ell^{2}=\left(1-K r^{2}\right)^{-1} \mathrm{~d} r^{2}+$ $r^{2} \mathrm{~d} \Omega^{2}$ representa o elemento de linha dos observadores comóveis, convenientemente representado por um sistema de coordenadas esféricas.

A expansão do universo define naturalmente dois sistemas de coordenadas: o sistema físico e o sistema comóvel. O sistema de coordenadas comóveis representa um observador em queda livre que, portanto, segue o fluxo da expansão sempre numa mesma posição comóvel $\boldsymbol{x}$. A posição física $\boldsymbol{l}$ desse observador fica determinada uma vez conhecida a lei de expansão do universo em termos do fator de escala

$$
\boldsymbol{l}=a(t) \boldsymbol{x} .
$$

Um exemplo típico de um observador comóvel é uma galáxia. Porém, se a galáxia tiver uma velocidade intrínseca e independente da expansão, sua posição comóvel também será uma função do tempo. Nesse caso a velocidade da galáxia é a soma das velocidades de expansão e intrínseca

$$
\begin{aligned}
\boldsymbol{v} & =H(t) \boldsymbol{l}+a(t) \dot{\boldsymbol{x}} \\
& =\boldsymbol{v}_{\mathrm{exp}}+\boldsymbol{v}_{\mathrm{int}}
\end{aligned}
$$

onde

$$
H \equiv \frac{\dot{a}}{a}
$$

é a função de Hubble. Ignorando por hora a velocidade intrínseca e considerando a função de Hubble como sendo aproximadamente constante, encontramos

$$
v=H_{0} l
$$

que é a conhecida lei de Hubble, válida para objetos suficientemente próximos do observador. A análise conjunta das medidas da RCF, medidas de luminosidade de supernovas do tipo Ia e da oscilação acústica de bárions na distribuição de galáxias mostram que [9]

$$
H_{0}=70,1 \pm 1,3 \mathrm{~km} \mathrm{~s}^{-1} \mathrm{Mpc}^{-1} .
$$

\footnotetext{
${ }^{1}$ Essa discussão não diz nada sobre a topologia do universo e, em princípio, existem várias possibilidades compatíveis com a hipótese de homogeneidade e isotropia. Novamente, são as observações que devem dizer qual é a topologia do universo em que vivemos [31].
} 


\subsubsection{Cinemática da Expansão}

Podemos tirar algumas conclusões gerais sobre os efeitos da expansão do universo antes mesmo de conhecer sua lei geral de expansão. Consideremos por exemplo a propagação de um fóton no espaço-tempo descrito por (1.2). Levando em conta que fótons viajam em geodésicas nulas caracterizadas por $\mathrm{d} s^{2}=0=\mathrm{d} \Omega^{2}$, segue de (1.2) que

$$
\int_{t}^{t_{0}} \frac{\mathrm{d} t^{\prime}}{a\left(t^{\prime}\right)}= \pm \int_{0}^{r} \frac{\mathrm{d} r^{\prime}}{\sqrt{1-K r^{\prime 2}}}
$$

onde a arbitrariedade da escolha do sinal deve ser feita de acordo com o sentido conveniente da propagação do fóton.

Como vimos, a distância comóvel $r$ é independente do tempo. Isso significa que dois pulsos emitidos em instantes $t_{0}$ e $t$ arbitrariamente próximos $\left(\operatorname{com} t_{0}>t\right)$ devem ser tais que

$$
\frac{\delta t_{0}}{a\left(t_{0}\right)}=\frac{\delta t}{a(t)}
$$

Em termos da freqüência e do comprimento de onda emitidos e observados, temos

$$
\lambda_{\mathrm{obs}}(t)=\frac{a\left(t_{0}\right)}{a(t)} \lambda_{\mathrm{em}}, \quad \nu_{\mathrm{obs}}(t)=\frac{a(t)}{a\left(t_{0}\right)} \nu_{\mathrm{em}} .
$$

Ou seja, a expansão do universo tem um impacto direto sobre as ondas eletromagnéticas, fazendo com que os comprimentos (freqüências) das ondas aumentem (diminuam) com o fator de escala se $a\left(t_{0}\right)>a(t)$. Essa conseqüência da expansão é fundamental e garante, entre outras coisas, que o espectro planckiano da RCF seja preservado durante a expansão do universo [30].

Normalmente os resultados que envolvem a expansão do universo são interpretados em termos do efeito Doppler e do parâmetro de redshift z, definido como

$$
1+z \equiv \frac{a\left(t_{0}\right)}{a(t)} .
$$

Se o comprimento de onda aumenta (diminui) dizemos que a onda sofreu um desvio para o vermelho (azul), ou um redshift (blueshift). Dessa forma, a definição acima nos permite escrever

$$
z=\frac{\lambda_{\mathrm{obs}}-\lambda_{\mathrm{em}}}{\lambda_{\mathrm{em}}}=v=H_{0} l
$$

que nada mais é que a lei de Hubble (1.6) reescrita à luz do efeito Doppler. O parâmetro de redshift é também muitas vezes usado como uma medida de tempo.

Duas quantidades de natureza cinemática e fundamentais em qualquer universo em expansão são o tempo e o raio de Hubble, definidos respectivamente como

$$
t_{H} \equiv H^{-1}, \quad R_{H}=c H^{-1}
$$

onde $c$ é a velocidade da luz. Para o valor atualmente conhecido do parâmetro de Hubble (1.7), essas quantidades valem

$$
t_{H} \approx 14 \times 10^{9} \text { anos }, \quad R_{H} \approx 4,27 \mathrm{Gpc}
$$


Embora não definam exatamente a idade e o tamanho do universo, essas quantidades dão uma noção dos valores típicos envolvidos nos modelos cosmológicos. De fato, o valor tido como a idade atual do universo é $t_{0}=(13,73 \pm 0,12) \times 10^{9}$ anos [9], enquanto que o raio de um universo contendo apenas matéria é $2 R_{H}$ (equação (1.32)).

\subsubsection{Dinâmica da expansão}

A dinâmica de evolução do universo, ou seja, a determinação da função $a(t)$, depende diretamente da especificação do seu conteúdo material. Essa relação de dependência é dada pela teoria da relatividade geral de Einstein

$$
R_{\mu \nu}-\frac{1}{2} g_{\mu \nu} R=8 \pi G T_{\mu \nu}
$$

que relaciona o conteúdo geométrico do universo $\left(R_{\mu \nu}-\frac{1}{2} g_{\mu \nu} R\right)$ ao seu conteúdo material $\left(T_{\mu \nu}\right)$. Em princípio, a teoria da relatividade geral não tem nada a dizer sobre a forma específica do tensor de energia-momento $T_{\mu \nu}$. Entretanto, o princípio cosmológico limita drasticamente a forma geral do mesmo, de modo que apenas o tensor de um fluido perfeito

$$
T_{\mu \nu}=(\rho+p) u_{\mu} u_{\nu}+p g_{\mu \nu}
$$

é compatível com as hipóteses de simetria. Além disso, tanto a densidade de energia $\rho$ como a pressão $p$ devem ser funções apenas do tempo, pois do contrário o universo não seria homogêneo. Na expressão acima, $u_{\mu}$ é o quadrivetor de velocidade do fluido perfeito e, no sistema de coordenadas comóvel, ele vale $u_{\mu}=(1,0,0,0)$.

Esses ingredientes nos permitem reescrever as equações de Einstein (1.15) para o tensor de um fluido perfeito (1.16) em termos da métrica (1.2). As equações resultantes são também chamadas de equações de Friedmann

$$
\begin{aligned}
H^{2} & =\frac{8 \pi G}{3} \rho-\frac{K}{a^{2}} \\
\frac{\ddot{a}}{a} & =-\frac{4 \pi G}{3}(\rho+3 p) .
\end{aligned}
$$

onde $H$ foi definido em (1.5). Essas equações são suplementadas pela equação de conservação do tensor $T_{\mu \nu}$

$$
\dot{\rho}+3 H(\rho+p)=0 .
$$

Do ponto de vista de graus de liberdade adicionais essa última equação é redundante, já que ela é uma conseqüência das identidades de Bianchi. Do ponto de vista operacional, no entanto, é por vezes conveniente substituir a equação (1.18) pela equação (1.19).

O sistema de equações (1.17-1.18) depende de 3 incógnitas $a, p$ e $\rho$. Sua solução portanto fica indeterminada a menos que eliminemos uma dessas incógnitas. Nesse ponto nem as equações de Einstein, nem as simetrias do espaço-tempo podem nos ajudar. No entanto, uma vez que estamos lidando com um fluido perfeito, podemos fazer uso de argumentos termodinâmicos/hidrodinâmicos para simplificar as equações. Normalmente o que se faz é assumir a existência de uma equação de estado entre a densidade de energia e a pressão do fluido

$$
p=w(\rho) \rho .
$$


Esse é o caso da maioria dos fluidos perfeitos utilizados em cosmologia. Em particular, o modelo padrão contempla a existência de radiação eletromagnética, matéria (bariônica e escura) e uma constante cosmológica como conteúdo de energia do universo. Esses três "fluidos" podem ser considerados como perfeitos, e cuja termodinâmica especifica as seguintes equações de estado

$$
w= \begin{cases}0 & \text { matéria bariônica/escura } \\ 1 / 3 & \text { radiação } \\ -1 & \text { constante cosmológica }\end{cases}
$$

Em geral, se $w=$ constante a solução da equação (1.19) é imediata

$$
\mathrm{d} \ln \rho=-3(1+w) \mathrm{d} \ln a \quad \rightarrow \quad \rho(a)=\rho_{0} a^{-3(1+w)}
$$

onde $\rho_{0}$ é a densidade atual de energia do fluido em questão. Para os casos particulares de radiação, matéria e constante cosmológica, temos respectivamente

$$
\rho(a)=\left\{\begin{array}{lll}
\rho_{0}^{m} a^{-3} & \text { se } \quad w=0 \\
\rho_{0}^{r} a^{-4} & \text { se } \quad w=1 / 3 \\
\rho_{0}^{\Lambda}=\text { const. } & \text { se } \quad w=-1
\end{array}\right.
$$

O comportamento geral dessas soluções é de fácil interpretação. Em primeiro lugar notemos que, quaisquer que sejam os valores atuais das constantes de proporcionalidade $\rho_{0}^{m}, \rho_{0}^{r}$ e $\rho_{0}^{\Lambda}$, a densidade de energia da radiação sempre domina a energia total do universo no infinito passado. O mesmo vale para a constante cosmológica no infinito futuro. Em segundo lugar, por ser uma densidade de energia, a função $\rho(a)$ é inversamente proporcional ao volume físico $V \sim a^{3}$. Como no caso da matéria o número de partículas $n_{p}$ se conserva, temos imediatamente que $n_{p} / V \sim a^{-3}$. O caso da radiação é diferente pois, como vimos, a freqüência (e portanto a energia da onda) é inversamente proporcional ao fator de escala: $\nu=E / h \sim a^{-1}$. Nesse caso, portanto, $E / V \sim a^{-4}$. Já a solução de constante cosmológica corresponde ao que de fato se espera de uma constante.

Num modelo mais realista do universo, esses três fluidos devem ser considerados simultaneamente, ou seja, $\rho=\rho_{m}+\rho_{r}+\rho_{\Lambda}$. Portanto a equação de Friedmann (1.17) pode ser reescrita na forma

$$
1=\Omega_{m}+\Omega_{r}+\Omega_{\Lambda}-\frac{K}{a^{2} H^{2}}
$$

onde introduzimos a densidade crítica de energia

$$
\rho_{\text {crit }} \equiv \frac{3 H^{2}}{8 \pi G}
$$

e a densidade relativa de cada espécie, $\Omega_{X} \equiv \rho_{X} / \rho_{\text {crit }}$, sendo $X=(m, r, \Lambda)$. Mais importante que uma solução analítica para $\rho(a)$ nesse caso é a constatação de que a densidade de energia total $\Omega_{\text {total }}=\Omega_{m}+\Omega_{r}+\Omega_{\Lambda}$ está intimamente ligada à curvatura do universo

$$
\frac{K}{a^{2} H^{2}}=\Omega_{\text {total }}-1
$$


ou seja, a curvatura da seção espacial do universo será positiva, nula ou negativa se e somente se a densidade de energia for maior, igual ou menor que 1, respectivamente

$$
\begin{aligned}
& \Omega_{\text {total }}>1 \longleftrightarrow K=1 \quad \text { (Universo fechado) } \\
& \Omega_{\text {total }}=1 \longleftrightarrow K=0 \quad \text { (Universo plano) } \\
& \Omega_{\text {total }}<1 \longleftrightarrow K=-1 \quad \text { (Universo aberto) }
\end{aligned}
$$

Os dados atuais da RCF mostram que $-0.0175<\frac{K}{a_{0}^{2} H_{0}^{2}}<0.0085$ com $95 \%$ de confiança estatística [9]. Isso parece sugerir uma coincidência improvável à primeira vista: por que dentre todos os valores possíveis para $K \in[-1,1]$ o universo escolheria exatamente $K=0$ ? Como veremos mais à frente, o modelo inflacionário prevê exatamente um universo plano. Esse dado portanto pode ser visto como uma das confirmações do paradigma inflacionário.

Se $K=0$ a equação (1.17) pode ser facilmente resolvida para o caso geral de um fluido com equação de estado constante

$$
a^{(1+3 w) / 2} \mathrm{~d} a=\frac{8 \pi}{3} \rho_{0} \mathrm{~d} t \quad \rightarrow \quad a(t)=a_{0} t^{2 / 3(1+w)} .
$$

Para os casos particulares de matéria, radiação e constante cosmológica, essa solução se reduz a

$$
a(t)=\left\{\begin{array}{lll}
a_{0}^{m} t^{2 / 3} & \text { se } & w=0 \\
a_{0}^{r} t^{1 / 2} & \text { se } & w=1 / 3 \\
a_{0}^{\Lambda} e^{H_{\Lambda} t} & \text { se } & w=-1
\end{array}\right.
$$

onde $H_{\Lambda} \equiv \sqrt{8 \pi G \rho_{0}^{\Lambda} / 3}$. Essas soluções mostram que enquanto para a matéria e a radiação temos $\ddot{a}<0$, para o caso de uma constante cosmológica, $\ddot{a}>0$. A equação de estado negativa é fundamental para a modelagem do período inflacionário do universo, como veremos.

\subsubsection{Horizontes cosmológicos}

A noção de horizonte desempenha um papel crucial na construção da teoria inflacionária e, de modo geral, na cosmologia moderna. Um horizonte cosmológico é uma hipersuperfície no espaço-tempo definida por um observador $\mathcal{O}$ que divide eventos observáveis de eventos não observáveis. A condição que um dado evento tem de ser ou não ser observado por $\mathcal{O}$ está ligada à possibilidade desse observador, convenientemente posicionado na origem do sistema físico de coordenadas, detectar sinais luminosos oriundos de uma distância finita

$$
\chi=a(t) \int^{t} \frac{\mathrm{d} t^{\prime}}{a\left(t^{\prime}\right)} .
$$

Portanto a condição de existência de um horizonte está ligada à existência da integral acima sobre certo limites. Em particular podemos distinguir dois tipos de horizonte, chamados de horizonte de eventos e horizonte de particulas [32]. 


\section{Horizonte de eventos}

O horizonte de eventos é a região espaço-temporal que separa os eventos que foram, são e serão observados, dos eventos que jamais serão observados por um dado observador. Para o observador $\mathcal{O}$, a condição necessária e suficiente para que exista um horizonte de eventos é que a distância $\chi$ seja finita, ou seja, que

$$
a(t) \int_{t}^{\infty} \frac{\mathrm{d} t^{\prime}}{a\left(t^{\prime}\right)}<\infty .
$$

Assim, eventos posicionados em $\chi_{0}>\chi$ jamais serão observadas por $\mathcal{O}$. Num universo plano o fator de escala é tipicamente da forma $a(t)=a_{0} t^{n}$, nesse caso a condição de existência do horizonte de eventos fica garantida se e somente se $n>1$. Ou seja, num universo dominado por matéria $(n=2 / 3)$ ou radiação $(n=1 / 2)$ não existem eventos inobserváveis, por mais distantes que estejam do observador $\mathcal{O}$. Um exemplo importante de um universo com horizonte de eventos é o universo de de Sitter. Nesse caso o horizonte $\chi$ coincide com o raio de Hubble $H_{\Lambda}^{-1}$. Veremos mais adiante que durante o período inflacionário do universo o fator de escala cresce de forma aproximadamente exponencial, o que significa que $H_{\mathrm{inf}}^{-1} \approx$ constante. Quando esse for o caso usaremos os termos "horizonte de eventos" e "raio de Hubble" indiscriminadamente.

\section{Horizonte de partículas}

O horizonte de partículas definido pelo observador $\mathcal{O}$ é a região espacial que, num dado instante $t=t_{0}$, divide o universo em eventos já observados de eventos ainda não observados. A condição necessária e suficiente para que $\mathcal{O}$ possua um horizonte de partículas é que

$$
a(t) \int_{0}^{t} \frac{\mathrm{d} t}{a(t)}<\infty .
$$

Essa definição corresponde à nossa intuição de "universo observável" e está intimamente ligada à existência de uma singularidade inicial. Para o caso geral $a(t)=a_{0} t^{n}$, a condição de existência do horizonte de partículas é satisfeita se $n<1$. Nesse caso o raio do horizonte de partículas tem uma forma muito simples

$$
\chi(t)=\frac{n}{1-n} H^{-1}, \quad n<1
$$

e que é satisfeita tanto para o caso de um universo dominado por matéria $\left(\chi=2 H^{-1}\right)$ como por radiação $\left(\chi=H^{-1}\right)$.

Nesse ponto já podemos constatar um aspecto incômodo do modelo cosmológico padrão que será abordado com mais detalhes adiante. O problema é que, num universo dominado por matéria ou radiação, as perturbações lineares evoluem como ondas planas cujo comprimento de onda físico é dado por $\lambda_{f} \propto t^{n}$, com $n=(1 / 2,2 / 3)$. Essa lei de evolução, que claramente difere de $\chi(t)$, mostra que diferentes regiões que hoje têm as mesmas propriedades físicas e estatísticas nem sempre estiveram em contato causal, como mostra a Fig. 1.1. A ausência de contato causal no passado impede a existência de um mecanismo físico capaz de explicar por que diferentes regiões do céu têm hoje as mesmas propriedades. À primeira vista esse problema sugere um ajuste 
extremamente preciso das condições iniciais do universo. Mostraremos adiante como um período de inflação pode contornar esse problema.

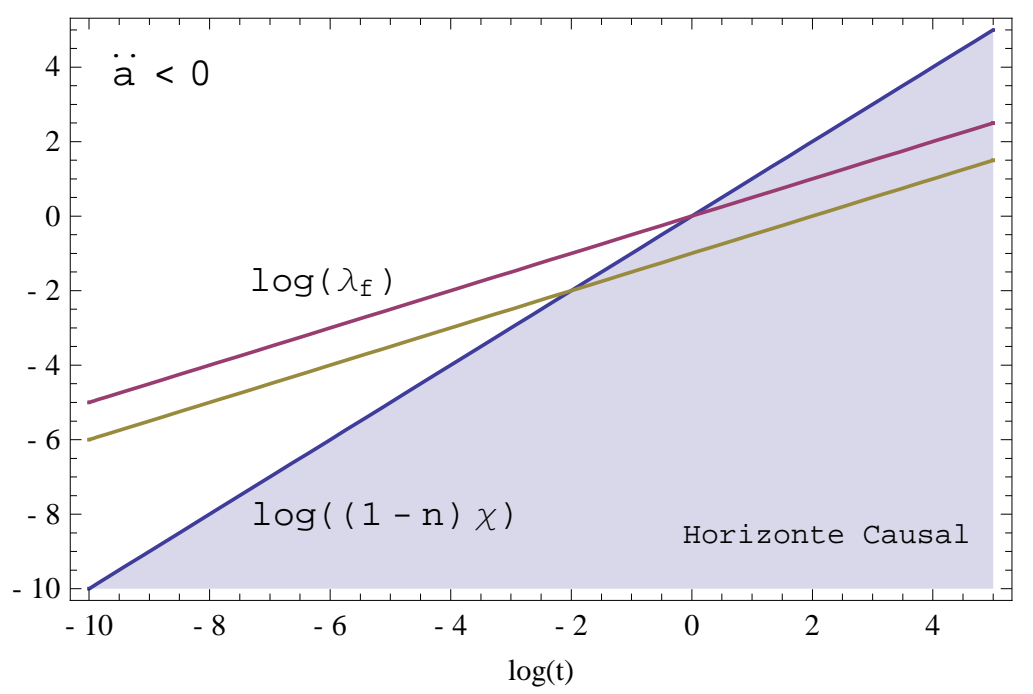

Figura 1.1: Evolução do logaritmo das perturbações lineares em um universo com horizonte de partículas. A figura corresponde a $n=2 / 3$.

\subsection{Big Bang e a origem da RCF}

A descrição geométrica que acabamos de apresentar não contempla todos os aspectos do modelo cosmológico padrão. Na verdade, grande parte do sucesso da cosmologia moderna se deve aos trabalhos de Gamow, Alpher, Herman e Bethe sobre as conseqüências da evolução térmica do universo. As previsões da abundância dos elementos leves [33] e da existência de uma radiação cósmica com espectro planckiano [34, 35] são hoje indissociáveis do modelo quente do Big Bang. Todas essas previsões resultam de uma física muito bem conhecida e testada em energias inferiores a alguns $\mathrm{MeV}$, física essa que é capaz de descrever, de maneira absolutamente conservadora, o universo tão jovem quanto $10^{-2} \mathrm{~s}$.

O modelo do Big Bang quente é baseado em diversas evidências observacionais de que o universo está em expansão (veja [36] para uma revisão). Isso naturalmente nos leva a supor que o universo tenha evoluído a partir de uma singularidade inicial onde a sua temperatura e energia eram arbitrariamente grandes. Como vimos, a própria dinâmica do fator de escala indica que o universo tenha uma singularidade em $t=0$. Além disso, se o universo é composto por fluidos de características termodinâmicas distintas, sua evolução dinâmica passará por períodos distintos. Em termos da densidade crítica de energia $\rho_{\text {crit }}=3 H^{2} / 8 \pi G$ e do parâmetro de redshift (1.11), as densidades relativas de energia da matéria, radiação e energia escura se comportam como

$$
\Omega_{m}(z)=\Omega_{0}^{m}(1+z)^{3}, \quad \Omega_{r}(z)=\Omega_{0}^{r}(1+z)^{4}, \quad \Omega_{\Lambda}(z)=\Omega_{0}^{\Lambda}(1+z)^{3\left(1+w_{\Lambda}\right)} .
$$

onde levamos em conta que a equação de estado da energia escura assume um valor constante entre $-1 \leq w_{\Lambda}<-1 / 3$. Portanto, quaisquer que sejam os valores atuais de 
$\Omega_{0}^{m}, \Omega_{0}^{r}$ e $\Omega_{0}^{\Lambda}$, o conteúdo de energia do universo é cronologicamente dividido em períodos de domínio da radiação, da matéria e da energia escura. O período de transição matéria/energia escura, definido pelo redshift

$$
1+z_{\Lambda}=\left(\frac{\Omega_{0}^{m}}{\Omega_{0}^{\Lambda}}\right)^{1 / 3 w_{\Lambda}}
$$

é o mais recente na história cósmica, tendo acontecido entre $0.3 \lesssim z_{\Lambda} \lesssim 1.3$, dependendo do valor exato de $w_{\Lambda}$. Em todo caso, é curioso que essa transição tenha ocorrido justamente após o período de formação de galáxias. A determinação precisa do redshift $z_{\Lambda}$ (ou, equivalentemente, de $w_{\Lambda}$ ) é hoje um problema em aberto e conhecido como problema da coincidência cósmica. Por outro lado a transição radiação/matéria é muito bem conhecida. Levando em conta que a contribuição atual da radiação é cerca de $0.01 \%$ da energia total, e que a matéria (bariônica e escura) contribui com cerca de $27 \%$, o redshift de transição equivale a

$$
1+z_{\text {eq }}=\frac{\Omega_{0}^{m}}{\Omega_{0}^{r}} \simeq 10^{4}
$$

Portanto, em $z>z_{\text {eq }}$ a energia do universo foi dominada pela energia de partículas relativísticas. Assim a relação entre temperatura e densidade de energia do universo nesse período pode ser determinada pela expressão conhecida

$$
\rho_{r}(T)=\sum_{i=1}^{n} \alpha_{i} g_{i}\left(\frac{\pi^{2}}{30}\right) T^{4}
$$

onde $n$ é o número de espécies de partículas relativísticas, $g_{*}$ representa o número de graus de liberdade efetivos (spin) de cada partícula e $\alpha_{i}=1,7 / 8$ respectivamente para bósons e férmions. Mais importante nesse caso é a dependência da radiação com a temperatura. Se comparada à lei de evolução $\rho_{r} \propto a^{-4}$ deduzida na seção anterior, concluímos que $T \propto a^{-1}$ ou, em termos do redshift

$$
T(z)=T_{0}(1+z)
$$

onde $T_{0}=2.75 \mathrm{~K}$ é a temperatura da radiação cósmica de fundo medida hoje.

Durante a era de pleno domínio da radiação, $z \gg 10^{4}$ e portanto $T \gg 10^{4} \mathrm{~K}$. Essa temperatura é alta o suficiente para ionizar, via espalhamento Compton, os átomos de hidrogênio e de hélio presentes no universo. Se essas colisões acontecerem numa taxa maior que a taxa de expansão do universo, então o sistema se comporta efetivamente como um plasma opaco cuja temperatura evolui como $T \propto a^{-1}$. De fato é isso o que ocorre. Se o universo é dominado por radiação então $a \propto t^{1 / 2}$ e a escala de tempo típica da expansão é $t_{\exp }=a / \dot{a}=2 t$. Por outro lado, o tempo típico de colisão entre as partículas é da forma $t_{\text {col }} \propto \lambda^{3} / n \sigma$, onde $\lambda \propto a$ é o caminho livre médio, $n$ é o número de partículas e $\sigma$ a seção de choque efetiva. Supondo que $n \sigma$ é constante vemos que $t_{\text {col }} \propto t^{2}$ e que portanto $t_{\text {col }} \ll t_{\text {exp }}$ para instantes suficientemente pequenos. À medida que o universo evolui a temperatura desse plasma vai diminuindo até o instante em que ela deixa de ser grande o suficiente para impedir que os elétrons se liguem aos prótons formando átomos neutros. Esse desacoplamento ocorre por volta de $z_{\text {dec }} \simeq 1090 \mathrm{ou}$, equivalentemente, $T_{\mathrm{dec}} \simeq 3.000 \mathrm{~K}$. Nesse instante a matéria se 
desacopla dos fótons e esses se propagam livremente pelo universo, dando origem à radiação cósmica de fundo. O "último" instante de interação dos fótons com a matéria delimita uma região conhecida como superfície de último espalhamento. É essa "região" que acreditamos medir com nossos satélites. Nessa descrição as flutuações de temperatura presentes na RCF correspondem aos diversos mecanismos que governam o plasma relativístico de fótons, bárions e matéria escura da era da radiação. Durante o desacoplamento, que ocorre por volta de $z_{\mathrm{dec}}=1090$, os detalhes desses mecanismos ficam gravados na temperatura e na polarização dos fótons, ambas passíveis de medida.

A temperaturas abaixo de $10^{10} \mathrm{~K}$ a energia do universo - aproximadamente $10 \mathrm{MeV}$ - é suficiente para que processos nucleares desencadeiem a nucleossíntese primordial dos elementos químicos. A descrição do universo até esse ponto é bastante confiável pois a física nessas escalas é muito bem conhecida [37]. Podemos no entanto ir além dessas escalas. A energia de operação dos aceleradores de partículas mais recentes, como por exemplo o acelerador de partículas LHC (Large Hadron Colider, na sigla inglesa), é da ordem de $10 \mathrm{TeV}$, o que equivale a uma temperatura de $10^{17} \mathrm{~K}$. Essas escalas são bem acomodadas pela teoria padrão das interações eletro-fracas, e com ela podemos descrever um universo tão jovem quanto $10^{-12} \mathrm{~s}$. Previsões acima desses valores vão se tornando mais e mais especulativas e, em alguma escala, a descrição correta da cosmologia deve depender essencialmente de um tratamento quântico e não-perturbativo da gravidade, ainda por ser desenvolvido.

\subsection{Alguns problemas}

O desenvolvimento de uma teoria quântica da gravitação - que supostamente nos permitirá estender o modelo do Big Bang para além da escala de Planck - é certamente o maior problema da física moderna. Entretanto, mesmo nas escalas acessíveis à nossa física atual o modelo do Big Bang apresenta alguns problemas. Como veremos esses problemas se resumem à peculiaridade das condições iniciais do universo compatíveis com o que hoje observamos.

\subsubsection{Problema da planura}

O problema da planura do universo ${ }^{2}$ consiste em explicar por que sua densidade total de energia é igual à densidade crítica de energia. Esse problema pode ser entendido como um problema de condições iniciais. Para entendê-lo melhor reescrevamos a equação de Friedmann (1.17) na forma

$$
\left(\Omega^{-1}-1\right) \frac{8 \pi G}{3} \rho a^{2}=-K
$$

onde $\Omega=\rho / \rho_{\text {crit. }}$. Da constância do parâmetro de curvatura, segue que

$$
\left.\left(\Omega^{-1}-1\right) \rho a^{2}\right|_{t=t_{\mathrm{i}}}=\left.\left(\Omega^{-1}-1\right) \rho a^{2}\right|_{t=t_{\mathrm{f}}}
$$

sendo que o lado esquerdo é avaliado em um instante anterior ao lado direito $\left(t_{\mathrm{i}}<t_{\mathrm{f}}\right)$. Se esses instantes forem escolhidos como sendo a era de igualdade entre a matéria e a

\footnotetext{
${ }^{2}$ Flatness Problem, em inglês.
} 
radiação, onde $\rho_{\text {eq }} \approx \rho_{0}^{m} a^{-3}$, e a era atual, teremos

$$
\frac{\left(\Omega_{\mathrm{eq}}^{-1}-1\right)}{\left(\Omega_{0}^{-1}-1\right)}=a_{\mathrm{eq}}=\left(1+z_{\mathrm{eq}}\right)^{-1} \text {. }
$$

onde tomamos, sem perda de generalidade, $a_{0}=1$. Esse mesmo raciocínio pode ser aplicado a algum instante arbitrário do passado, durante a era de pleno domínio da radiação, até o fim do domínio da radiação

$$
\frac{\left(\Omega^{-1}-1\right)}{\left(\Omega_{\mathrm{eq}}^{-1}-1\right)}=\left(\frac{a}{a_{\mathrm{eq}}}\right)^{2}=\left(\frac{T_{\mathrm{eq}}}{T}\right)^{2} .
$$

Comparando (1.40) a (1.41) e usando (1.37), encontramos

$$
\frac{\left(\Omega^{-1}-1\right)}{\left(\Omega_{0}^{-1}-1\right)}=\left(1+z_{\mathrm{eq}}\right)\left(\frac{T_{0}}{T}\right)^{2} .
$$

Podemos agora ter uma atitude conservadora e assumir que $T=10^{17} \mathrm{~K}$, o que corresponde à escala de energia das interações eletro-fracas atualmente acessível aos nossos aceleradores. De modo mais otimista, podemos assumir que a relatividade geral funciona bem até a escala de Planck, onde $T=10^{32} \mathrm{~K}$. Em ambos os casos, se as observações atuais nos dizem que $\Omega_{0}=1 \pm \epsilon$, com $\epsilon \lesssim 1$, então o valor da densidade total de energia no passado deve ter sido

$$
\Omega= \begin{cases}1 \pm 10^{-30} & \text { escala das interações eletro-fracas } \\ 1 \pm 10^{-60} & \text { escala de Planck }\end{cases}
$$

Em termos do parâmetro de densidade de curvatura $\Omega_{K} \equiv K / a^{2} H^{2}$, isso significa que se $\Omega_{K} \approx 0$ hoje, então $\Omega_{K} \sim 10^{-30}, 10^{-60}$ no passado. A menos, é claro, que $K=0$. Como não temos nenhum motivo a priori para essa escolha, a conclusão imediata do resultado acima é que deveria existir um ajuste extremamente fino das condições iniciais do universo para que ele fosse hoje como o observamos.

\subsubsection{Problema do horizonte}

O problema do horizonte consiste em explicar por que a RCF em diferentes regiões do céu tem a mesma temperatura. A origem desse problema já foi esboçada quando abordamos a questão do horizonte de partículas em um universo dominado por matéria e/ou radiação em $§ 1.1 .3$.

Digamos que a superfície de último espalhamento dos fótons esteja localizada num raio $\eta_{\mathrm{dec}}=\int^{t_{\mathrm{dec}}} \mathrm{d} t / a$ de distância. A distância comóvel $D$ que um fóton percorre desde essa superfície até nossos instrumentos em $\eta=\eta_{0}$, ignorando qualquer processo de interação nessa trajetória, é dada por

$$
D=\int_{\eta_{\mathrm{dec}}}^{\eta_{0}} \mathrm{~d} \eta=\eta_{0}-\eta_{\mathrm{dec}}
$$

Isso significa que um comprimento comóvel $s$ qualquer, projetado na superfície de último espalhamento corresponde a um ângulo $\theta=s /\left(\eta_{0}-\eta_{\text {dec }}\right)$ da esfera celeste. 
Dentro dessa superfície o comprimento de onda máximo de uma perturbação do plasma formado pelos fótons e pelas partículas livres é $s=c_{s} \eta_{\mathrm{dec}}$, onde $c_{s}=1 / \sqrt{3(1+R)}$ é a velocidade do som do plasma e $R=3 \rho_{b} / 4 \rho_{r}$ é a razão entre a densidade de energia dos bárions e dos fótons. Tipicamente esse número é menor que um. Tomando $R=0$ numa primeira aproximação, vemos que o ângulo $\theta$ vale

$$
\theta=c_{s} \frac{\eta_{\mathrm{dec}}}{\eta_{0}-\eta_{\mathrm{dec}}}=\frac{1}{\sqrt{3}}\left(\frac{T_{0}}{T_{\mathrm{dec}}}\right)^{1 / 2} \approx 1^{\circ}
$$

onde consideramos que $K=0$, por simplicidade, e usamos a relação (1.37) juntamente com $a \propto \eta^{2} \propto T^{-1}$ e $z_{\text {dec }}=1090$. Isso significa que a escala típica de contato causal entre as perturbações antes do desacoplamento dos fótons com a matéria é da ordem de um grau ${ }^{3}$. O número de tais regiões dentro do volume formado pela superfície de último espalhamento é da ordem de

$$
N=\frac{4 \pi D^{3} / 3}{4 \pi(D \theta / 2)^{3} / 3}=\frac{8}{\theta^{3}} \sim 10^{6}
$$

Ou seja, a esfera celeste contém aproximadamente $10^{6}$ dessas regiões. Todavia a temperatura da radiação cósmica de fundo em toda a esfera celeste vale $2.75 \mathrm{~K}$, com flutuações de apenas $0.001 \%$ em torno desse valor. Parece portanto difícil explicar como um número tão grande de regiões distintas e causalmente desconexas possam ter exatamente a mesma temperatura. Dentro do modelo padrão isso só é possível se as condições iniciais de cada uma das $10^{6}$ regiões forem minuciosamente ajustadas para um mesmo valor. Entretanto é importante enfatizar que esse problema é uma conseqüência direta da nossa hipótese de que o universo é o mesmo em todos os pontos. Ou seja, não se trata de um problema que conflita com a teoria mas sim de entender esse número do ponto de vista de diferentes realizações do universo.

\subsubsection{Origem das perturbações}

É evidente que as hipóteses de simetria do princípio cosmológico devem ser vistas como uma primeira aproximação do universo, uma vez que desvios de homogeneidade e isotropia são observados tanto na distribuição de galáxias como na distribuição de temperatura da RCF. Contudo, se tais desvios forem pequenos, podemos tratá-los com uma teoria de perturbações adequada. O problema é que, num universo em expansão desacelerada, a teoria de perturbações cosmológicas é pouco eficaz no sentido de explicar a amplitude das estruturas observadas com base apenas em argumentos de instabilidade gravitacional. Tipicamente, os modos de Fourier das flutuações primordiais (de cuja natureza ignoraremos por enquanto) se comportam como um sistema harmônico forçado

$$
\delta \ddot{\phi}_{k}+3 H \delta \dot{\phi}_{k}+\frac{k^{2}}{a^{2}} \delta \phi_{k}=0
$$

onde a expansão do universo atua como uma força externa igual a $-3 H \delta \dot{\phi}$. Se o comprimento de onda de uma perturbação for pequeno $\left(a^{-1} k \gg H\right)$, então a solução

\footnotetext{
${ }^{3}$ Note também que esse ângulo seria ainda menor se $0 \leq R \leq 1$. Nos casos em que o universo não é plano, esse ângulo sofre uma alteração. No entanto, para valores tais que $-0.1 \lesssim \Omega_{K} \lesssim 0.1, \theta$ continua sendo da ordem de um grau.
} 
da equação acima é

$$
\delta \phi_{k}(t)=\frac{C_{k}}{a} \exp \left[ \pm \mathrm{i} k \int \frac{\mathrm{d} t}{a(t)}\right]
$$

Já as perturbações com comprimentos de ondas maiores que o raio de Hubble $\left(a^{-1} k \ll\right.$ $H$ ) apresentam como solução um modo constante e um modo decrescente

$$
\delta \phi_{k}(t)=A_{k}+B_{k} \int \frac{\mathrm{d} t}{a(t)^{3}} .
$$

O comportamento das amplitudes das perturbações nesses limites é bastante geral e independe da lei de evolução temporal do fator de escala. Todavia, num universo em expansão desacelerada os comprimentos de onda das perturbações crescem numa taxa menor que a taxa de expansão do universo (Fig. 1.1). Dessa forma as amplitudes das perturbações irão oscilar e decair com o fator de escala se os seus comprimentos de onda forem inicialmente menores que o raio de Hubble (solução (1.48)). Perturbações com comprimentos de onda maiores que o raio de Hubble permanecem com suas amplitudes "congeladas" (solução (1.49)) até o momento em que $\lambda \sim H^{-1}$. A partir daí suas amplitudes irão novamente oscilar e decair.

Esse quadro oferece duas explicações para as perturbações que hoje têm amplitudes da ordem de $10^{-5}$, como é o caso das flutuações de temperatura da RCF. A primeira delas consiste em assumir que as amplitudes das perturbações foram arbitrariamente grandes no passado e diminuíram com a evolução do universo até valores compatíveis com as observações atuais. Essa solução torna inviável a aplicação de qualquer teoria linear de perturbações cosmológicas onde se assume explicitamente que a amplitude das perturbações sejam pequenas (menores que um). A segunda solução consiste em associar as perturbações observáveis à regiões causalmente desconexas do universo. Porém, como vimos, essa solução requer que as condições iniciais das perturbações sejam minuciosamente ajustadas a um mesmo valor no passado. Em ambos os casos as explicações parecem pouco satisfatórias. Além disso, tão importante quanto a descrição da amplitude das estruturas é a descrição estatística das suas propriedades. Sabemos hoje que as flutuações de temperatura da RCF seguem uma distribuição de probabilidade gaussiana com enorme grau de precisão. A distribuição espacial dessas flutuações é fracamente dependente da escala cosmológica, apresentando um desvio para a parte vermelha do espectro de apenas 4\% [9]. Dentro do modelo quente do Big Bang a existência desse espectro é usualmente postulada e não tem explicação a partir de primeiros princípios. 


\section{CAPÍTULO 2}

As questões que acabamos de apresentar não representam de fato um problema pois não estão em conflito com a teoria da relatividade geral, nem com o princípio cosmológico. Pelo contrário, alguns dos "problemas" apresentados acima se mostram como uma manifestação clara das nossas hipóteses. O fato dessas questões serem incômodas tem uma explicação muito simples: o objeto de estudo da cosmologia é único e irreproduzível; portanto, um modelo cosmológico completo deve explicar não só o universo observável mas também quais são as condições iniciais "típicas" que dão origem a esse universo. Como vimos, as condições iniciais previstas pelo modelo do Big Bang são extremamente peculiares e "pouco prováveis" do ponto de vista de realizações aleatórias do universo. Embora a definição do que é ou não típico e provável num sistema de uma única realização seja bastante questionável, a escolha cega de um conjunto particular de condições iniciais também o é. Devemos somar a isso o fato de que questões sobre o que é ou não "natural" em cosmologia raramente levam a conseqüências que sejam observáveis. Nesse sentido a construção de uma teoria de condições iniciais é duplamente importante pois pode não só esclarecer tais arbitrariedades como também nos guiar na construção de observáveis cosmológicos.

A teoria da inflação como uma teoria de condições iniciais para o universo foi originalmente proposta por Alan H. Guth em 1981 [10], com base em um modelo de unificação das forças fundamentais. Nesse modelo a inflação é causada por um campo escalar, o inflaton. Inicialmente preso num mínimo relativo do seu potencial, o inflaton age como uma constante cosmológica causando a expansão acelerada do universo. O período de inflação acaba quando o inflaton atinge o mínimo absoluto do potencial por meio de um processo de tunelamento. Esse cenário, conhecido como oldinflation, foi logo abandonado quando percebeu-se que a inflação não poderia ocorrer em todos os pontos do universo simultaneamente, mas apenas em "bolhas" isoladas que eram ou completamente desprovidas de matéria, ou com distribuição de matéria completamente inomogênea e anisotrópica [38]. Tal cenário foi aprimorado por Andrei Linde [39], Andreas Albrecht e Paul Steinhardt [40] através de modelos onde o inflaton rola lentamente em direção ao mínimo do seu potencial. Conhecido hoje como new- 
inflation, esse modelo também teve de ser abandonado, pois em nenhum modelo efetivo o inflaton era capaz de estabelecer o equilíbrio termodinâmico com os outros campos de matéria, o que tornaria inviável o estabelecimento das transições de fases cosmológicas. Entretanto a idéia de um período de rolagem lenta do inflaton é ainda central nos modelos de inflação, pois é nesse período onde são geradas as perturbações de densidade que dão origem às estruturas de grandes escalas do universo (veja [41] para um revisão atualizada da história da inflação e do seu status atual).

A explicação da origem das estruturas do universo não foi um problema que inicialmente motivou a idéia da inflação. Entretanto, no mesmo ano da publicação de A. Guth, V. Mukhanov e G. Chibisov mostraram que a teoria quântica de perturbações do universo primordial pode explicar a origem microscópica das grandes estruturas do universo [42]. Tal idéia foi logo desenvolvida em uma gama de trabalhos que evidenciaram a importância do período inflacionário para a origem das estruturas de grandes escalas [43, 44, 45]. Essa característica do modelo é de interesse central nas pesquisas atuais sobre a inflação.

\subsection{Resolvendo os problemas do Big Bang}

A idéia central por trás de todos os modelos inflacionários é que um período de expansão acelerada do universo, anterior ao período de domínio da radiação, pode explicar os problema da planura e do horizonte. Assim, a condição necessária para que a inflação ocorra é

$$
\frac{\ddot{a}}{a}=-\frac{4 \pi G}{3}(\rho+3 p)>0 ;
$$

condição essa que não é satisfeita por nenhum tipo de matéria ordinária, nem radiação, pois requer que a condição forte de energia seja violada:

$$
\rho+3 p<0 \quad \rightarrow \quad w<-\frac{1}{3}
$$

Uma quantidade central em todos esses modelos é o número de e-folds, definido de forma a medir o quanto o universo se expande durante a inflação

$$
N \equiv \ln \frac{a_{\mathrm{f}}}{a_{\mathrm{i}}}=\int_{t_{\mathrm{i}}}^{t_{\mathrm{f}}} H \mathrm{~d} t
$$

onde $a_{\mathrm{i}}$ e $a_{\mathrm{f}}$ são respectivamente o fator de escala no início e no fim do período de expansão. Vejamos agora como esse período de expansão acelerada pode resolver os problemas do Big Bang.

\subsubsection{Problema da planura}

O problema da planura, assim como os outros problemas do Big Bang, é uma conseqüência imediata de um universo cujo conteúdo material é sempre atrativo. Notemos que

$$
|\Omega-1|=\frac{|K|}{a^{2} H^{2}}
$$


enquanto que

$$
\lim _{t \rightarrow \infty} a^{2} H^{2}=\lim _{t \rightarrow \infty} \dot{a}^{2}=0 .
$$

Esse limite resulta do fato de que, num universo atrativo, a velocidade de expansão tende à zero. Isso mostra que o valor $\Omega=1$ é um ponto fixo instável do modelo. Visto dessa forma fica fácil entender o porque da inflação. Se o fator de escala tiver um crescimento exponencial no passado então $\Omega$ tende a 1 com uma enorme precisão. Dessa forma a subseqüente evolução do universo garante que $\Omega_{0}=1$, sem a necessidade de ajustes finos das condições iniciais. Supondo que a física é bem descrita a temperaturas ligeiramente abaixo da temperatura de Planck, então

$$
\left.\left(\Omega^{-1}-1\right) a^{2} \rho\right|_{t_{\mathrm{i}}}=\left.10^{-60}\left(\Omega_{0}^{-1}-1\right) a^{2} \rho\right|_{t_{\mathrm{f}}} .
$$

Portanto o problema estará resolvido se $a_{\mathrm{f}} \gg 10^{30} a_{\mathrm{i}}$, o que equivale a

$$
N \gtrsim 70 \text { e-folds }
$$

\subsubsection{Problema do horizonte}

Um período de expansão exponencial no passado do universo implica a existência de um "horizonte efetivo" de eventos constante e proporcional ao raio de Hubble $H^{-1}$. Dizemos que o horizonte é efetivo pois o mesmo existirá apenas durante a inflação. Dessa forma as perturbações que hoje crescem numa taxa menor que a taxa de expansão do universo tiveram durante a inflação um período de crescimento exponencial, enquanto o raio de causalidade permanecia constante. Isso pode explicar por que as $10^{6}$ regiões causalmente desconexas do céu têm hoje as mesmas características físicas. Dado um domínio causalmente conexo do universo jovem, o efeito da inflação é o de homogeneizar a distribuição de matéria dessa região, esticando seus comprimentos de onda para além do horizonte causal, como mostra a Fig. 2.1.

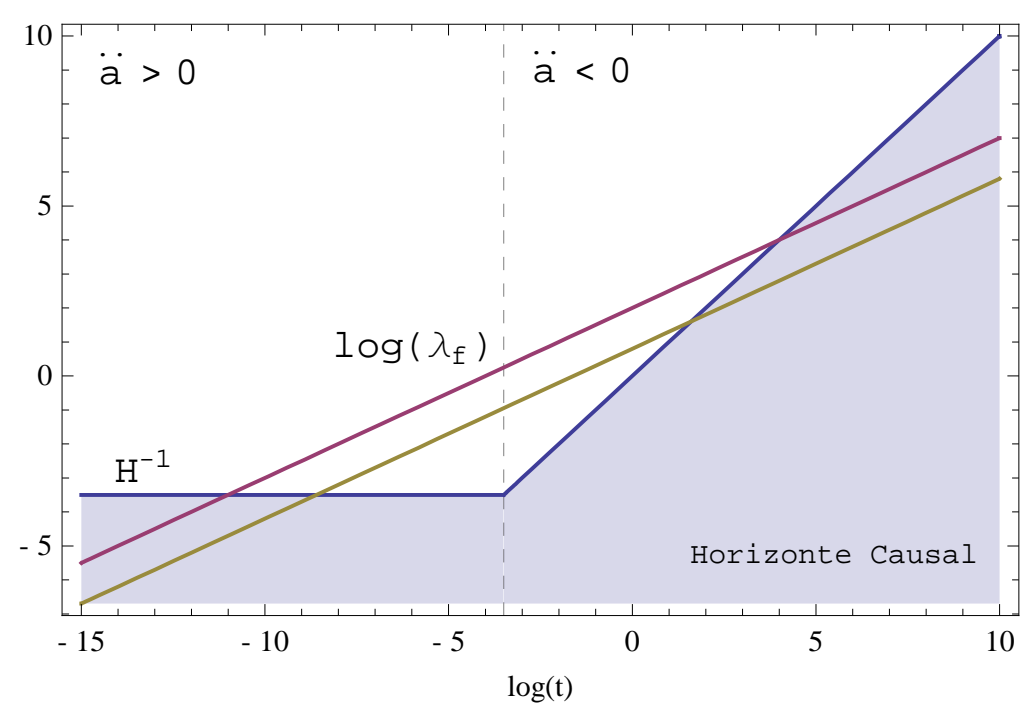

(a) Fig(1): ad dofi usdf

Figura 2.1: Evolução do logaritmo do comprimento de onda e do horizonte causal durante $(\ddot{a}>0)$ e depois $(\ddot{a}<0)$ da inflação. Note que durante a inflação o raio de Hubble $\left(H^{-1}\right)$ permanece constante. 
Portanto, a solução inflacionária para o problema do horizonte é muito simples: as regiões desconexas do céu têm hoje as mesmas propriedades pois estiveram em contato causal no passado. Em outras palavras, o universo observável corresponde a um único domínio causal que é inflado a escalas arbitrariamente grandes. A condição necessária para que o problema do horizonte seja resolvido é que as maiores escalas observáveis hoje $\left(H_{0}^{-1}\right)$ sejam menores que o raio de Hubble durante a inflação $\left(H_{\mathrm{inf}}^{-1}\right)$, ou seja

$$
H_{0}^{-1} \lesssim \frac{a_{0}}{a_{\mathrm{i}}} H_{\mathrm{inf}}^{-1}=\frac{a_{0}}{a_{\mathrm{f}}} e^{N} H_{\mathrm{inf}}^{-1}
$$

onde usamos (2.3) e $a_{0}=a\left(t_{0}\right)$. Isolando $N$ na expressão acima, obtemos

$$
N \gtrsim \ln \left(\frac{a_{\mathrm{f}} H_{\mathrm{inf}}}{a_{0} H_{0}}\right)=\ln \left(\frac{T_{0} H_{\mathrm{inf}}}{T_{\mathrm{f}} H_{0}}\right) .
$$

Para se ter uma idéia do número de e-folds, vamos assumir que a inflação termina na escala $\mathrm{GUT}^{1}$ de unificação das forças fundamentais, onde $T_{\mathrm{GUT}} \sim 10^{29} \mathrm{~K}$ e $H_{\text {inf }} \sim$ $T_{\text {GUT }}^{2} / M_{\text {Planck }} \sim 10^{56} \mathrm{~km} / \mathrm{s} / \mathrm{Mpc}$. Usando $H_{0} \sim 10^{1} \mathrm{~km} / \mathrm{s} / \mathrm{Mpc}$ e $T_{0} \sim 1 \mathrm{~K}$, encontramos

$$
N \gtrsim 60 \text { e-folds. }
$$

Rigorosamente falando essa estimativa é pouco precisa, pois não leva em conta as diferentes fases do universo após a inflação. No entanto, os modelos inflacionários mais simples prevêem um número de e-folds várias ordens de grandeza acima desse valor, dos quais nossas observações só permitem observar os últimos 60 - 70 e-folds.

\subsubsection{Origem das perturbações}

A teoria inflacionária das perturbações cosmológicas será rigorosamente construída no $\S 3$. Porém, antes de entrarmos nos detalhes técnicos, devemos mencionar a importância da inflação para a origem das grandes estruturas cosmológicas, pois esse período pode explicar não somente a amplitude dessas estruturas como também suas propriedades estatísticas. Notemos que enquanto a expansão do universo for exponencial, o raio de Hubble permanecerá constante, ao passo que o comprimento de onda das perturbações crescerá proporcionalmente ao fator de escala. Dessa forma, a amplitude das perturbações que nascem dentro do horizonte (solução (1.49)) diminuem até o instante em que seus comprimentos de onda se igualam ao raio de Hubble (Fig. 2.2). A partir daí as flutuações permanecem com suas amplitudes constantes, enquanto seus comprimentos de onda são esticados exponencialmente. Terminada a inflação essas perturbações seguem sua evolução normal e reentram o horizonte de partículas quando $\lambda \sim H^{-1}$, essencialmente com a mesma amplitude inicial. Outro detalhe importante é que a constância do raio de Hubble implica que a escala do horizonte será sempre a mesma, $k=a H$; portanto as perturbações que deixam o horizonte o fazem sempre na mesma escala. Em outras palavras o espectro das perturbações que resulta do período inflacionário é um espectro invariante de escala. Finalmente, desde que a inflação ocorra num instante posterior ao tempo de Planck podemos aplicar os métodos usuais da teoria de campos para quantizar as perturbações cosmológicas. Em particular, se as perturbações desse período forem pequenas, a teoria de campos prevê que as mesmas devem obedecer uma distribuição de probabilidades gaussiana.

\footnotetext{
${ }^{1}$ Grand Unification Theory.
} 
(a)

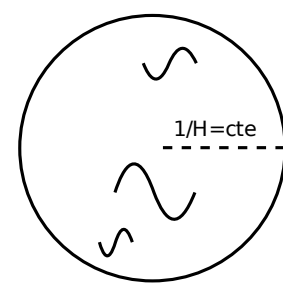

(b)

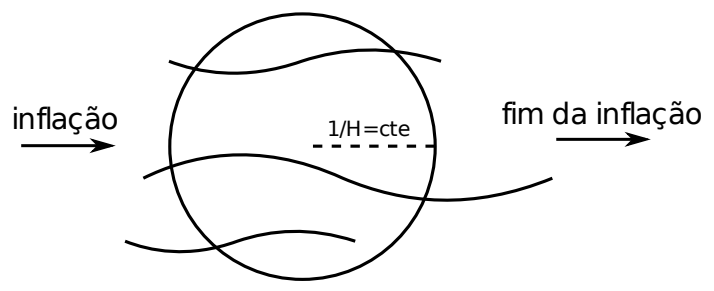

(c)

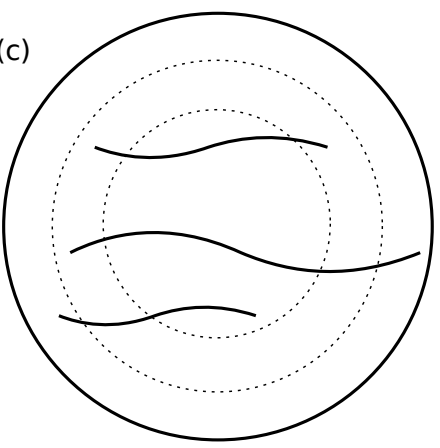

Figura 2.2: Representação esquemática da evolução das pertubações durante e depois da inflação. (a) comprimentos de onda crescem e amplitudes diminuem, enquanto o raio de Hubble permanece constante. (b) comprimentos de onda crescem e amplitudes se congelam, o raio de Hubble permanece fixo. (c) com o fim da inflação o raio de Hubble passa a crescer numa taxa muito maior que os comprimentos de onda (Fig. 1.1). Dizemos portanto que as perturbações "reentram" no universo.

\subsection{O Inflaton}

A maneira mais fácil de se implementar a inflação é através de um campo escalar real $\phi$ que evolui sob a ação de uma função potencial $V(\phi)$. Nos modelos mais simples a ação do campo é dada por

$$
S[\phi]=-\int \mathrm{d}^{4} x \sqrt{-g}\left[\frac{1}{2} \partial^{\mu} \phi \partial_{\mu} \phi+V(\phi)\right] .
$$

Essa descrição é também a mais atraente do ponto de vista teórico, pois a existência de campos escalares no universo jovem pode ser acomodada dentro da teoria padrão das partículas elementares. Variando a ação com relação à métrica encontramos o tensor de energia-momento do campo

$$
T_{\nu}^{\mu}=\partial^{\mu} \phi \partial_{\nu} \phi-\left(\frac{1}{2} \partial^{\lambda} \phi \partial_{\lambda} \phi+V(\phi)\right) \delta_{\nu}^{\mu} .
$$

Numa primeira aproximação supomos que o inflaton é um campo homogêneo $(\phi=$ $\phi(t))$ e que atua efetivamente como um fluido perfeito cuja energia e a pressão são dados por

$$
\rho_{\phi}=\frac{1}{2} \dot{\phi}^{2}+V(\phi), \quad p_{\phi}=\frac{1}{2} \dot{\phi}^{2}-V(\phi) .
$$

Em termos dessa abordagem a condição (2.1) necessária para que o universo possua um período inflacionário se traduz em $\dot{\phi}^{2}<V(\phi)$. Essa condição pode facilmente ser obtida através de algumas restrições sobre o potencial $V(\phi)$, como veremos adiante.

A dinâmica do inflaton num universo em expansão fica completamente determinada pelas duas equações de Friedmann

$$
\begin{aligned}
H^{2} & =\frac{8 \pi G}{3}\left(\frac{1}{2} \dot{\phi}^{2}+V(\phi)\right) \\
\ddot{a} & =-\frac{8 \pi G}{3}\left(\dot{\phi}^{2}-V(\phi)\right)
\end{aligned}
$$


onde levamos em conta que o termo de curvatura $K / a^{2}$ é subdominante num universo em expansão inflacionária, e tende rapidamente a zero após alguns e-folds de expansão. A equação de continuidade do inflaton é a equação de Klein-Gordon

$$
\ddot{\phi}+3 H \dot{\phi}+V_{,_{\phi}}=0, \quad V_{, \phi} \equiv \frac{\mathrm{d} V}{\mathrm{~d} \phi}
$$

e segue também de $\dot{\rho}_{\phi}+3 H\left(\rho_{\phi}+p_{\phi}\right)=0$. Derivando a equação (2.12) e utilizando (2.14), chegamos a uma relação simples entre o campo e a derivada da função de Hubble

$$
\dot{H}=-4 \pi G \dot{\phi}^{2}
$$

e que será bastante útil a seguir.

\subsubsection{Regime de rolagem lenta}

Rigorosamente, a escolha do potencial $V(\phi)$ deve ser guiada por alguma teoria fundamental da física de altas energias. Entretanto as características gerais da inflação devem ser independentes da forma específica de cada potencial e pode ser analisada através da chamada aproximação de rolagem lenta do campo ${ }^{2}$. Essa aproximação consiste em supor que a energia cinética do inflaton é inicialmente muito menor que sua energia potencial

$$
\dot{\phi}^{2} \ll V(\phi) .
$$

Isso garante que a expansão do universo seja aproximadamente exponencial, pois a "equação de estado" do inflaton nesse caso se comporta efetivamente como a equação de estado de uma constante cosmológica: $w_{\phi}=p_{\phi} / \rho_{\phi} \approx-1$. Na prática, é costume supor também que a aceleração do campo seja muito menor que sua velocidade

$$
\ddot{\phi} \ll 3 H \dot{\phi} \text {. }
$$

Juntas, as aproximações (2.16) e (2.17) implicam que

$$
\begin{aligned}
H^{2} & \simeq \frac{8 \pi G}{3} V(\phi) \\
3 H \dot{\phi} & \simeq-V_{\phi}
\end{aligned}
$$

e podem ser vistas como restrições à forma do potencial $V(\phi)$. Tomando-se a derivada de $H^{2}$ e $\dot{\phi}$ com relação ao tempo, usando (2.19) e em seguida (2.15), verifica-se que

$$
\frac{M_{\mathrm{p}}^{2}}{3}\left(\frac{V,,_{\phi}}{V}\right)^{2} \ll 1, \quad \frac{M_{\mathrm{p}}^{2}}{3}\left|\frac{V, \phi \phi}{V}\right| \ll 1
$$

onde $M_{\mathrm{p}}=1 / \sqrt{8 \pi G}$ é a massa de Planck em unidades naturais.

As condições de rolagem lenta do campo (2.16) e (2.17) podem ser convenientemente introduzidas através de dois novos parâmetros $\epsilon$ e $\delta$, definidos de modo que

\footnotetext{
${ }^{2}$ Slow-roll condition, em inglês.
} 


$$
\epsilon \equiv-\frac{\dot{H}}{H^{2}}=\frac{1}{2 M_{\mathrm{p}}^{2}} \frac{\dot{\phi}^{2}}{H^{2}}, \quad \delta \equiv-\frac{\ddot{\phi}}{H \dot{\phi}}
$$

sendo que o regime de rolagem lenta será obtido se $\epsilon, \delta \ll 1$. Dessas definições segue que

$$
\dot{\epsilon}=2 H\left(\epsilon^{2}-\epsilon \delta\right),
$$

portanto o parâmetro $\epsilon$ pode ser considerado constante desde que $\epsilon, \delta \ll 1$. Notemos contudo que essa restrição é mais forte que a condição (2.1). Com efeito, derivando $H$ com relação ao tempo e rearranjando os termos, encontramos

$$
\frac{\ddot{a}}{a}=H^{2}(1-\epsilon)=-\frac{1}{6 M_{\mathrm{p}}^{2}} \rho_{\phi}\left(1+\omega_{\phi}\right)
$$

sendo que na última igualdade usamos (2.1). Portanto a condição necessária para que a inflação exista pode ser vista como uma condição sobre o parâmetro $\epsilon$ :

$$
\omega_{\phi}=-1+\frac{2}{3} \epsilon<-\frac{1}{3} \quad \rightarrow \quad \epsilon<1 .
$$

Na prática, os modelos de campos escalares possuem soluções atratoras que garantem que $\epsilon, \delta \ll 1$ durante boa parte da evolução do campo. Essa característica dos modelos com campos escalares será explorada em §2.2.4.

Por vezes será útil reescrever o número de e-folds em termos do parâmetro $\epsilon$. De (2.3) segue que

$$
N=\int_{t_{\mathrm{i}}}^{t_{\mathrm{f}}} \frac{H}{\dot{\phi}} \mathrm{d} \phi=\frac{1}{\sqrt{2} M_{\mathrm{p}}} \int_{\phi_{\mathrm{f}}}^{\phi_{\mathrm{i}}} \frac{\mathrm{d} \phi}{\sqrt{\epsilon}}
$$

onde usamos $\dot{H}=H,_{\phi} \dot{\phi}$ e $H,_{\phi} / H=\sqrt{2 \epsilon} / M_{\mathrm{p}}$. Essa expressão nos permitirá calcular a taxa de expansão do universo durante o regime de rolagem lenta do campo.

\subsubsection{Equações em tempo conforme}

\section{Definições gerais}

Em todas as equações anteriores utilizamos o tempo físico $t$, que nada mais é que o tempo próprio dos observadores em queda livre com a expansão do universo. Muitas vezes é conveniente reescrever as equações em termos do tempo conforme $\eta$, definido como

$$
\eta \equiv \int \frac{\mathrm{d} t}{a(t)} .
$$

O motivo do nome "tempo conforme" fica evidente quando reescrevemos a métrica de Friedmann (1.2) em termos desse parâmetro. No caso plano $(K=0)$, a métrica resultante é conforme à métrica de Minkowski

$$
\mathrm{d} s^{2}=a(\eta)^{2}\left(-\mathrm{d} \eta^{2}+\mathrm{d} x^{2}+\mathrm{d} y^{2}+\mathrm{d} z^{2}\right) .
$$

Isso significa que as propriedades espaço-temporais de qualquer objeto geométrico no espaço de Minkowski é preservada no espaço de Friedmann. Por exemplo, se um vetor for tipo-tempo em Minkowski, também o será em Friedmann. 
Para qualquer função $f$ dependente do tempo, a relação entre as derivadas no tempo físico e conforme são obtidas através de

$$
\dot{f}=\frac{f^{\prime}}{a} .
$$

Em particular, a relação entre o parâmetro de Hubble e sua derivada nos tempos físico e conforme será usada freqüentemente. Definindo

$$
\mathcal{H} \equiv \frac{a^{\prime}}{a}
$$

temos que

$$
H=\frac{\mathcal{H}}{a}, \quad \dot{H}=\frac{1}{a^{2}}\left(\mathcal{H}^{\prime}-\mathcal{H}^{2}\right) .
$$

Usando essas relações podemos reescrever as equações de Friedmann (1.17-1.18) em termos do tempo conforme

$$
\mathcal{H}^{2}=\frac{8 \pi G}{3} a^{2} \rho-K, \quad \mathcal{H}^{\prime}=-\frac{4 \pi G}{3}(\rho+3 p) a^{2} .
$$

A equação de continuidade mantém a mesma forma funcional no tempo conforme

$$
\rho^{\prime}+3 \mathcal{H}(\rho+p)=0
$$

e portanto as soluções (1.22) ainda se aplicam. Já a dinâmica do fator de escala é diferente. Para o caso $K=0$ com $w=$ constante, as soluções (1.27) das equações de Friedmann ficam

$$
a(\eta)=\left\{\begin{array}{lll}
a_{0} \eta^{2} & \text { se } & w=0 \\
a_{0} \eta & \text { se } & w=1 / 3 \\
a_{0} \eta^{-1} & \text { se } & w=-1
\end{array}\right.
$$

Para os casos de matéria e radiação, o intervalo $t \in[0, \infty)$ é mapeado em $\eta \in[0, \infty)$. No entanto, se $w=-1$ a expansão é exponencial e o intervalo $t \in[0, \infty)$ será mapeado em $\eta \in(-\infty, 0)$.

\section{Inflação em tempo conforme}

A teoria das perturbações cosmológicas que apresentaremos no capítulo §3 será feita em termos do tempo conforme $\eta$. Para tanto será útil conhecer as expressões das quantidades inflacionárias em termos desse parâmetro. No tempo conforme, a densidade de energia e pressão do inflaton são

$$
\rho_{\phi}=\frac{\phi^{\prime 2}}{2 a^{2}}+V(\phi) \quad \text { e } \quad p_{\phi}=\frac{\phi^{\prime 2}}{2 a^{2}}-V(\phi) .
$$

Em função destas as equações de Friedmann e de Klein-Gordon são dadas respectivamente por

$$
\mathcal{H}^{2}=\frac{8 \pi G}{3}\left(\frac{\phi^{\prime 2}}{2}+a^{2} V(\phi)\right), \quad \mathcal{H}^{\prime}=-\frac{8 \pi G}{3}\left(\phi^{\prime 2}-a^{2} V(\phi)\right)
$$


e

$$
\phi^{\prime \prime}+2 \mathcal{H} \phi^{\prime}+a^{2} V_{\phi}=0 .
$$

Particularmente úteis serão as expressões para os parâmetros de rolagem lenta no tempo conforme. Das definições feitas em (2.21), segue que

$$
\epsilon=-\frac{1}{2 M_{\mathrm{p}}^{2}} \frac{\phi^{\prime 2}}{\mathcal{H}^{2}}, \quad \delta=1-\frac{\phi^{\prime \prime}}{\phi^{\prime} \mathcal{H}}
$$

e que

$$
\epsilon^{\prime}=2 \mathcal{H}\left(\epsilon^{2}-\epsilon \delta\right)
$$

Essa última propriedade dos parâmetros de rolagem lenta é muito útil, pois nos permite expandir o próprio parâmetro $\eta$ em função de $\epsilon$ e $\delta$. Com a ajuda de uma integral por partes, podemos mostrar que

$$
\eta=\int \frac{\mathrm{d} a}{a \mathcal{H}}=-\frac{1}{\mathcal{H}}+\int \epsilon \frac{\mathrm{d} a}{a \mathcal{H}} .
$$

Se $\epsilon \ll 1$ então, pela equação (2.37), $\epsilon$ também será constante, logo

$$
\eta=-\frac{1}{\mathcal{H}}(1+\epsilon)+\mathcal{O}(2)
$$

\subsubsection{Inflação Caótica}

Um modelo inflacionário bem conhecido e muito simples é o modelo de inflação caótica, introduzido por Andrei Linde no começo da década de oitenta [46]. Nesse modelo o potencial inflacionário é dado por uma função quadrática

$$
V(\phi)=\frac{m^{2} \phi^{2}}{2}
$$

onde $m$ é a massa do campo. Em linhas gerais, a idéia é que o campo, inicialmente com um valor $\phi_{\mathrm{i}}$, desça lentamente o potencial em direção ao seu mínimo. Durante esse processo a aproximação de rolagem lenta é satisfeita e o universo se expande de maneira quase exponencial. A inflação acaba quando a aproximação (2.16) deixa de valer e o campo passa a oscilar no fundo do seu potencial. Nesse processo de oscilação a energia do campo é convertida em partículas que reaquecerão o universo, dando início ao período de domínio da radiação. A figura abaixo mostra o comportamento do campo nesse modelo.
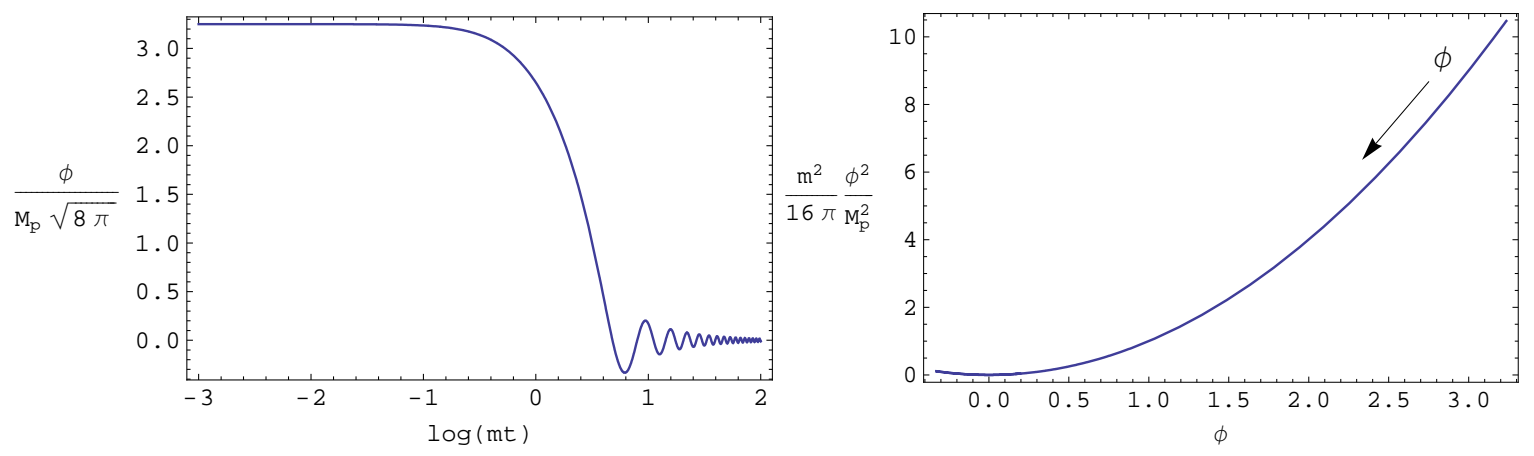

Figura 2.3: Comportamento do inflaton no modelo de inflação caótica: evolução dinâmica (esquerda) e função potencial (direita). 
Para que o potencial quadrático satisfaça as condições de suavidade (2.20) é necessário que o valor inicial do campo seja muito maior que a massa de Planck

$$
\phi \gg M_{\mathrm{p}}
$$

Essa condição de modo algum representa um problema para a nossa descrição semiclássica do universo, pois a energia do inflaton permanecerá abaixo da escala de Planck desde que $m \ll M_{\mathrm{p}}$. Assumindo que as condições de rolagem lenta sejam satisfeitas, teremos

$$
3 H \dot{\phi}=-m^{2} \phi, \quad H=\sqrt{\frac{1}{6}} \frac{m}{M_{\mathrm{p}}} \phi .
$$

A equação para $\dot{\phi}$ é facilmente integrável e fornece

$$
\phi(t)=\phi_{\mathrm{i}}-m M_{\mathrm{p}} \sqrt{\frac{2}{3}} t
$$

onde $\phi_{\mathrm{i}}=\phi\left(t_{\mathrm{i}}\right)$. Essa expressão nos permite calcular o número de e-folds durante toda a fase de rolagem lenta do campo. Considerando que esse regime termina no instante em que $\epsilon \lesssim 1$, e notando que para o potencial quadrático temos

$$
\epsilon=\frac{2 M_{\mathrm{p}}^{2}}{\phi^{2}}
$$

vemos que o valor mínimo do campo compatível com a aproximação de rolagem lenta é $\phi \gtrsim \sqrt{2} M_{\mathrm{p}}$. De (2.25) segue que o número de e-folds será

$$
N=\frac{1}{4} \frac{\phi_{\mathrm{i}}^{2}}{M_{\mathrm{p}}^{2}}-\frac{1}{2}
$$

Como vimos a solução para os problemas do horizonte e da planura do universo requerem um valor de $N \gtrsim 70$. No modelo de inflação caótica isso pode ser obtido desde que $\phi_{\mathrm{i}} \simeq 16,6 M_{\mathrm{p}}$.

\subsubsection{Regime Atrator}

Sendo a inflação uma teoria de condições iniciais, é natural que nos perguntemos quais são as condições iniciais que dão origem à inflação. Com razão, a dependência da fase inflacionária de um conjunto específico de condições iniciais tiraria toda a credibilidade do modelo, visto que nesse caso seria necessário reconstruir uma teoria de condições iniciais para a inflação, uma outra teoria de condições iniciais para essa última, ad infinitum. Felizmente, esse problema pode ser evitado na maioria dos modelos inflacionários com campos escalares e, em particular, para o modelo de inflação caótica. Isso acontece pois o sistema de equações (2.12) e (2.14) possui trajetórias atratoras para onde a evolução do sistema converge, como mostra a figura abaixo. 


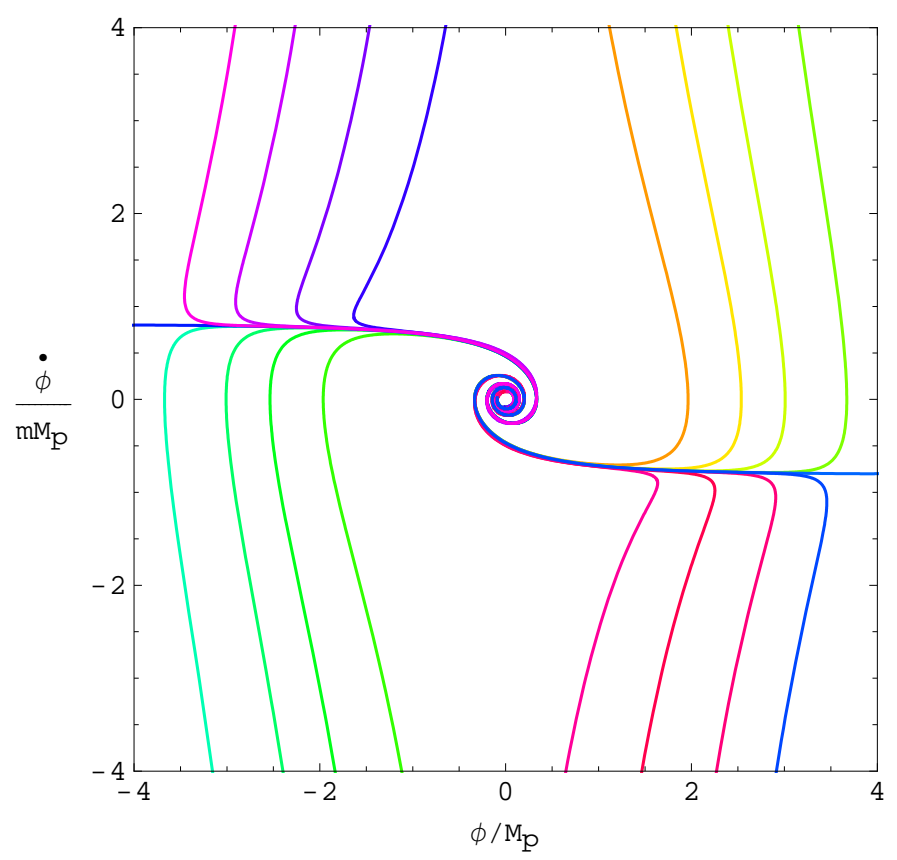

Figura 2.4: Diagrama de fase do inflaton para o modelo de inflação caótica. As diferentes curvas representam diferentes pares de condições iniciais $(\phi, \dot{\phi})$. Note que todas elas convergem para as curvas (aproximadamente) horizontais em torno de $\dot{\phi}=0$, onde o regime de rolagem lenta é estabelecido. A inflação acaba em $(\phi, \dot{\phi}) \approx(0,0)$.

A figura mostra também que as soluções atratoras são tais que $m^{-2} \dot{\phi}^{2} \ll \phi^{2}$, e portanto o regime de rolagem lenta é naturalmente estabelecido, quaisquer que sejam as condições $\left(\phi_{\mathrm{i}}, \dot{\phi}_{\mathrm{i}}\right)$. Veremos adiante que essa é uma característica robusta dos modelos inflacionários que permanece mesmo no caso em que o espaço-tempo de fundo não é isotrópico.

\subsection{Quantização de um modelo protótipo}

Nessa seção iremos analisar a origem das flutuações quânticas num modelo de inflação construído a partir de um campo escalar livre sobre o espaço-tempo de de Sitter. Embora não se trate de um modelo realista, visto que não iremos acoplar o inflaton ao campo gravitacional, a quantização desse modelo é bastante instrutiva pois ilustra as principais características dos modelos inflacionários efetivos. Além disso a apresentação que faremos aqui servirá de introdução ao cálculo formal das perturbações cosmológicas inflacionárias que será apresentado em $\S 4$.

Consideremos a ação de uma campo escalar canônico e de massa $m$

$$
S=-\frac{1}{2} \int d^{4} x \sqrt{-g}\left(g^{\mu \nu} \partial_{\mu} \phi \partial_{\nu} \phi+m^{2} \phi^{2}\right)
$$

evoluindo sobre um espaço-tempo que é descrito pela métrica

$$
g_{\mu \nu}=a^{2}(\eta) \operatorname{diag}(-1,+1,+1,+1), \quad a(\eta)=-(H \eta)^{-1}
$$

onde $H=$ constante e $\eta \in(-\infty, 0)$ é o tempo conforme. Nesse caso estamos implicitamente assumindo que a massa do campo é suficientemente pequena a ponto de não 
contribuir para a dinâmica do fator de escala. Substituindo $\phi$ por $v=a \phi$ em (2.46) e efetuando uma integração por partes ${ }^{3}$ chegamos à expressão canônica

$$
S=\frac{1}{2} \int d \eta d^{3} x\left[\left(v^{\prime}\right)^{2}-\left(\partial_{i} v\right)^{2}+m_{\mathrm{ef}}^{2} v^{2}\right]
$$

onde a massa efetiva

$$
m_{\mathrm{ef}}=\left(2 H^{2}-m^{2}\right) /\left(H^{2} \eta^{2}\right)
$$

é uma função do tempo pois leva em conta o fato de estarmos trabalhando em um espaço-tempo dinâmico. Através da ação (2.48) podemos identificar o par de variáveis canônicas $\left(v, \pi_{v}\right)$ do sistema, sendo que $\pi_{v}=\delta S / \delta v=v^{\prime}$. O processo de quantização consiste em promover essas variáveis a operadores $\left(\hat{v}, \hat{\pi}_{v}\right)$ sujeitos às regras de comutação

$$
\left[\hat{v}(\eta, \boldsymbol{x}), \hat{\pi}_{v}(\eta, \boldsymbol{y})\right]=\mathrm{i} \delta^{(3)}(\boldsymbol{x}-\boldsymbol{y}),
$$

sendo que todos os outros comutadores são iguais a zero. Geralmente esses operadores são reescritos em termos dos operadores de criação e aniquilação, $\hat{a}_{\boldsymbol{k}}$ e $\hat{a}_{\boldsymbol{k}}^{\dagger}$, do espaço de Fock

$$
\hat{v}(\eta, \boldsymbol{x})=\int \frac{\mathrm{d}^{3} k}{(2 \pi)^{3 / 2}}\left[v_{k}(\eta) \hat{a}_{\boldsymbol{k}} e^{\mathrm{i} \boldsymbol{k} \cdot \boldsymbol{x}}+v_{k}^{*}(\eta) \hat{a}_{\boldsymbol{k}}^{\dagger} e^{-\mathrm{i} \boldsymbol{k} \cdot \boldsymbol{x}}\right], \quad \hat{\pi}=\hat{v}^{\prime}
$$

os quais satisfazem as regras de comutação

$$
\left[\hat{a}_{\boldsymbol{k}}, \hat{a}_{\boldsymbol{q}}^{\dagger}\right]=\delta^{(3)}(\boldsymbol{k}-\boldsymbol{q}), \quad\left[\hat{a}_{\boldsymbol{k}}, \hat{a}_{\boldsymbol{q}}\right]=0=\left[\hat{a}_{\boldsymbol{k}}^{\dagger}, \hat{a}_{\boldsymbol{q}}^{\dagger}\right]
$$

e definem o estado de vácuo como sendo o vetor $|0\rangle$ do espaço de Fock tal que $\hat{a}_{\boldsymbol{k}}|0\rangle=0$, $\forall \boldsymbol{k} \in \mathbb{R}^{3}$. Em vista das regras (2.50) e (2.52), temos também que

$$
v_{k}(\eta) v_{k}^{*}(\eta)-v_{k}^{\prime}(\eta) v_{k}^{*}(\eta)=i
$$

Essa condição de normalização nada mais é que o Wronskiano das soluções. Através dele podemos fixar a amplitude das perturbações do universo primordial de maneira compatível com o princípio de incerteza de Heisenberg. Dessa forma podemos calcular o espectro de potência das perturbações e compará-lo às observações de uma maneira totalmente livre de ambigüidades.

Para calcular o espectro de potência em todos os seus limites, é importante conhecer a solução clássica dos modos $v_{k}(\eta)$. Esses modos obedecem a equação de um oscilador harmônico com uma frequência que depende do tempo

$$
v_{k}^{\prime \prime}+\omega^{2}(\eta) v_{k}=0, \quad \omega^{2}(\eta)=k^{2}-m_{\mathrm{ef}}^{2}
$$

No limite de pequenos comprimentos de onda, definido por $k^{2} \gg m_{\text {ef }}^{2}$, a equação de movimento se reduz a

$$
v_{k}^{\prime \prime}+k^{2} v_{k}=0
$$

cuja solução é

$$
v_{k}=c_{1} e^{\mathrm{i} k \eta}+c_{2} e^{-\mathrm{i} k \eta}
$$

\footnotetext{
${ }^{3}$ Supondo, é claro, que os campos sejam tais que se anulam no infinito.
} 
Esta solução é de fato esperada, dado que em pequenas escalas o espaço de de Sitter se assemelha ao espaço de Minkowski e, portanto, as soluções da equação de movimento são ondas planas. Para grandes comprimentos de onda, onde $k^{2} \ll m_{\text {ef }}^{2}$, a equação (2.54) torna-se

$$
v_{k}^{\prime \prime}-\frac{2 \beta}{\eta^{2}} v_{k}=0, \quad \beta \equiv \frac{2 H^{2}-m^{2}}{2 H^{2}} .
$$

Supondo que $m \ll H$, a solução da equação acima é dada pela soma de um modo crescente e outro decrescente

$$
v_{k}=c_{1} a+c_{2} a^{-2}
$$

O modo decrescente pode ser desprezado pois ele tende a zero à medida que $\eta \rightarrow 0$. Já o modo $v_{k} \propto a$ apresenta características mais interessantes. Da definição $v_{k}=a \phi_{k}$ segue que, nesse limite, $\phi_{k}$ tende a uma constante. Portanto, existe uma região comóvel, definida por $k \simeq m_{\mathrm{ef}}$, que distingue a física dos modos que estão dentro $\left(k / m_{\mathrm{ef}} \gg 1\right)$ da física dos modos que estão fora da mesma $\left(k / m_{\mathrm{ef}} \ll 1\right)$. Se a massa do campo fosse nula, a região física seria dada por $a k=2 a /|\eta|=2 H$. Trata-se portanto de uma região análoga ao "diâmetro de Hubble", $2 H$. Flutuações que vivem dentro dessa região se comportam como um campo escalar no espaço-tempo de Minkowski. À medida que o tempo evolui o comprimento de onda físico das flutuações, que cresce proporcionalmente ao fator de escala, atinge e eventualmente ultrapassa o horizonte. Fora do horizonte as flutuações se "congelam" e permanecem constantes. Esse quadro é aproximado para campos de massa pequena $(m \ll H)$ e exato para campos sem massa.

A solução geral da equação (2.54), válida em qualquer limite e para qualquer massa, é

$$
v_{k}=\sqrt{-\eta}\left[c_{1} H_{\nu}^{(1)}(-k \eta)+c_{2} H_{\nu}^{(2)}(-k \eta)\right] .
$$

onde $H_{\nu}^{(1,2)}$ são as funções de Hankel de primeira e segunda espécies e

$$
\nu^{2}=\frac{9}{4}-\frac{m^{2}}{H^{2}}
$$

As constantes da solução devem ser fixadas através da condição de normalização (2.53). Uma solução mais simples consiste em analisar o limite das funções de Hankel para pequenos comprimentos de onda (veja eq. (A.1) do Apêndice A)

$$
H_{\nu}^{(1,2)}(x \gg 1) \simeq \sqrt{\frac{2}{\pi x}} \exp \left[ \pm \mathrm{i}\left(x-\frac{\pi \nu}{2}-\frac{\pi}{4}\right)\right], \quad x=-k \eta .
$$

Usualmente, a solução de onda plana usada na quantização de campos no espaço de Minkowski é a solução proporcional a $\frac{1}{\sqrt{2 k}} e^{-i k \eta}$. Façamos então

$$
c_{1}=\sqrt{\frac{\pi}{4}} e^{\mathrm{i}\left(\nu+\frac{1}{2}\right) \frac{\pi}{2}}, \quad c_{2}=0 .
$$

Portanto a solução geral normalizada é

$$
v_{k}(\eta)=\frac{\sqrt{-\pi \eta}}{2} e^{\mathrm{i}\left(\nu+\frac{1}{2}\right) \frac{\pi}{2}} H_{\nu}^{(1)}(-k \eta)
$$




\subsubsection{Espectro de potências}

Definidas as variáveis usuais da quantização canônica podemos calcular a função de correlação de dois pontos, ou função de Green, que no espaço real é definida por

$$
\left\langle\hat{v}(\eta, \boldsymbol{x}) \hat{v}\left(\eta^{\prime}, \boldsymbol{y}\right)\right\rangle \equiv\left\langle 0\left|\hat{v}(\eta, \boldsymbol{x}) \hat{v}\left(\eta^{\prime}, \boldsymbol{y}\right)\right| 0\right\rangle .
$$

Na prática, essa definição é mais conveniente quando tomada no espaço de Fourier. De $(2.51)$ e (2.64), segue que

$$
\left\langle\hat{v}(\eta, \boldsymbol{x}) \hat{v}\left(\eta^{\prime}, \boldsymbol{y}\right)\right\rangle=\int \frac{d^{3} k}{(2 \pi)^{3}} G\left(k, \eta, \eta^{\prime}\right) e^{\mathrm{i} \boldsymbol{k} \cdot(\boldsymbol{x}-\boldsymbol{y})},
$$

onde

$$
G\left(k, \eta, \eta^{\prime}\right)=v_{k}(\eta) v_{k}^{*}\left(\eta^{\prime}\right)
$$

é a função de Green no espaço de Fourier. O espectro de potências das perturbações é definido em termos da função de Green calculada em tempos iguais e correspondentes ao instante em que os modos $k$ cruzam o horizonte de Hubble

$$
2 \pi^{2} k^{-3} \mathcal{P}_{v}(k)=\lim _{\eta \rightarrow \eta^{*}} G\left(k, \eta, \eta^{\prime}\right)=\left.\left|v_{k}(\eta)\right|^{2}\right|_{\eta^{*}} .
$$

Nesta expressão, o índice $\eta^{*}$ indica o instante em que os modos $k$ cruzam o horizonte definido por $k=m_{\mathrm{ef}}$.

Será interessante agora calcular o espectro de potências do campo original $\phi=$ $v / a$ no limite de grandes comprimentos de onda pois, por hipótese, é nesse limite que as perturbações geradas durante a inflação reentram o horizonte de partículas, contribuindo para a formação das estruturas atualmente observáveis. No limite em que $k \eta \ll 1$, usando a expansão (A.2), temos

$$
\mathcal{P}_{\phi}=2^{2 \nu-3}\left[\frac{\Gamma(\nu)}{\Gamma(3 / 2)}\right]^{2}\left(\frac{H}{2 \pi}\right)^{2}(-k \eta)^{3-2 \nu} .
$$

Do ponto de vista observacional, o espectro de potências é muitas vezes parametrizado por uma potência dos modos de Fourier: $\mathcal{P}_{\phi} \propto k^{n_{s}-1}$; desse modo o indice espectral escalar, $n_{s}$, especifica a dependência das perturbações com a escala

$$
n_{s}-1 \equiv \frac{\mathrm{d} \ln \mathcal{P}_{\phi}}{\mathrm{d} \ln k}=3-2 \nu .
$$

Supondo novamente que a massa do campo é muito menor que a taxa de expansão do universo, encontramos

$$
n_{s}-1 \simeq \frac{m^{2}}{3 H^{2}} .
$$

Isso nos mostra que a dependência da escala adquirida pelas perturbações é uma conseqüência direta do fato do campo escalar ter uma massa. Em particular, $n_{s}>1 \mathrm{e}$ a potência das perturbações se concentra com maior intensidade na banda ultravioleta do espectro. Dizemos nesse caso que o espectro é levemente "azul". Na maioria dos modelos efetivos de inflação o espectro resultante é levemente vermelho. No caso de um campo sem massa $\left(n_{s}=1\right)$, o espectro é dito invariante de escala, também conhecido como espectro de "Harrison-Zel'dovich" [47, 48]. 


\section{CAPÍTULO 3}

\section{Teoria Isotrópica de Pertubações Cosmológicas}

A teoria cosmológica delineada nos capítulos anteriores baseia-se em grande medida numa abordagem exata e independente de aproximações, o que só é possível graças às hipóteses simplificadores de homogeneidade e isotropia do princípio cosmológico. A teoria que resulta dessa abordagem funciona muito bem em escalas superiores a algumas centenas de megaparsecs, onde as hipóteses de simetria do universo são observacionalmente justificadas [28]. A rigor, no entanto, essa teoria é incompleta e deve ser vista apenas como uma primeira aproximação do universo em que vivemos. Estruturas complexas e claramente assimétricas, tais como galáxias, aglomerados de galáxias, "vazios", filamentos de matéria e mesmo as flutuações de temperatura da RCF são uma manifestação clara de que o princípio cosmológico só é válido em média. Podemos refinar o modelo supondo que as equações exatas dos capítulos anteriores fornecem uma descrição média do universo, sobre a qual devemos incluir pequenos desvios na distribuição de matéria e também na geometria do espaço-tempo. Esses desvios podem ser quantificados por meio de uma teoria de perturbações cosmológicas, a qual iremos descrever neste capítulo.

A teoria das perturbações cosmológicas é hoje o carro-chefe das pesquisas em cosmologia. Sua aplicação é vasta, podendo ser encontrada tanto em tratamentos fenomenológicos como em formulações puramente teóricas do universo. Existem duas abordagens para essa teoria, sendo uma newtoniana e outra relativística. A teoria newtoniana é geralmente usada para descrever perturbações tardias do universo com comprimentos de onda pequenos, tipicamente menores que o raio de Hubble. Dado que nossos objetivos consistem em entender a origem das perturbações com os maiores comprimentos de onda possíveis originadas no universo primordial, teremos que nos concentrar na teoria relativística das perturbações cosmológicas [49, 50].

\subsection{Perturbações Lineares}

A teoria da relatividade geral é uma teoria de caráter intrinsecamente não-linear, onde matéria e geometria estão acopladas por meio das equações de Einstein. Nesse qua- 
dro, pequenas flutuações nas distribuições de matéria e energia do universo acarretam pequenos desvios sobre a métrica do espaço-tempo. O caráter "auto-interagente" da teoria garante que a recíproca também seja válida: desvios da geometria geram desvios na matéria, ou seja

$$
T_{\mu \nu}+\delta T_{\mu \nu} \longleftrightarrow g_{\mu \nu}+\delta g_{\mu \nu}
$$

Se tais desvios assumirem valores pequenos comparados aos seus análogos nãoperturbados, ou seja, se $\left|\delta T_{\mu \nu}\right| \ll\left|T_{\mu \nu}\right|$ e $\left|\delta g_{\mu \nu}\right| \ll\left|g_{\mu \nu}\right|$, podemos linearizar as equações de Einstein, obtendo assim equações para as perturbações da geometria e da matéria. Em linhas gerais, o procedimento consiste em partir da decomposição geral da métrica

$$
g_{\mu \nu} \rightarrow g_{\mu \nu}+\delta g_{\mu \nu}, \quad g^{\mu \nu} \rightarrow g^{\mu \nu}-\delta g^{\mu \nu}
$$

e separar todas as quantidades que compõem o lado esquerdo das equações de Einstein em suas partes homogêneas e perturbadas. Assim, a combinação apropriada das quantidades $\Gamma_{\mu \nu}^{\lambda}+\delta \Gamma_{\mu \nu}^{\lambda}, R_{\mu \nu}+\delta R_{\mu \nu}, \ldots$, fornece as equações de Einstein linearizadas

$$
\delta R_{\mu \nu}-\frac{1}{2} g_{\mu \nu} \delta R-\frac{1}{2} \delta g_{\mu \nu} R=\kappa \delta T_{\mu \nu}, \quad \kappa \equiv 8 \pi G
$$

Todo esse procedimento depende, é claro, de parametrizações adequadas das flutuações da métrica e do tensor de energia-momento que levem em conta as simetrias do espaço-tempo de fundo.

\subsubsection{Perturbação da métrica}

A maneira mais geral de incluir perturbações lineares sobre a métrica de FriedmannRobertson-Walker (1.2) é através da seguinte parametrização

$$
\mathrm{d} s^{2}=a^{2}(\eta)\left[-(1+2 A) \mathrm{d} \eta^{2}+2 B_{i} \mathrm{~d} \eta \mathrm{d} x^{i}+\left(\gamma_{i j}+h_{i j}\right) \mathrm{d} x^{i} \mathrm{~d} x^{j}\right]
$$

onde $\gamma_{i j}$ é a parte espacial da métrica de FRW em coordenadas cartesianas. Esse ansatz, assim como qualquer métrica simétrica, contém 10 graus de liberdade, sendo 1 grau da função escalar $A, 3$ graus do campo vetorial $B_{i}$ e 6 graus de liberdade do tensor simétrico $h_{i j}$. Essa decomposição, portanto, é completamente geral.

Por simplicidade, e também motivados pelas observações atuais que sugerem que vivemos num universo de seção espacial plana [9], iremos nos restringir nesse trabalho à perturbações sobre uma métrica de fundo espacialmente plana. Nesse caso

$$
\gamma_{i j}=\delta_{i j}
$$

onde $\delta_{i j}$ é a delta de Kronecker. Assim sendo, os índices espaciais de uma quantidade perturbada qualquer será, em primeira ordem, manipulado com essa métrica.

\subsubsection{Perturbação do inflaton}

Durante a fase inflacionária do universo o inflaton é, por hipótese, o responsável pelo conteúdo de energia do universo. Sua perturbação pode ser parametrizada na forma

$$
\phi(\eta, \boldsymbol{x}) \rightarrow \phi(\eta)+\delta \phi(\eta, \boldsymbol{x})
$$

e depende de um único grau de liberdade adicional. 


\subsection{Decomposição EVT}

As equações linearizadas de Einstein que procuramos são equações dinâmicas para os graus de liberdade perturbativos da métrica e do tensor de energia-momento. Se o espaço-tempo de fundo onde se desenvolvem as perturbações for descrito pela métrica de FRW, então os modos escalares, vetoriais e tensoriais (EVT) das perturbações se desacoplam em primeira ordem, evoluindo de maneira independente uns dos outros. A decomposição em modos EVT, introduzida por J. Bardeen na década de 80 [51], facilita muito os cálculos das perturbações, pois permite que cada modo seja tratado isoladamente, de acordo com sua natureza.

Comecemos com a decomposição das funções introduzidas em (3.4). A função $A$ é, por construção, um escalar. Sua decomposição portanto é trivial. Já o vetor $B_{i}$ pode ser decomposto na forma

$$
B_{i}=\partial_{i} B+\bar{B}_{i}
$$

sendo que o modo $\bar{B}_{i}$ satisfaz a condição de transversalidade

$$
\partial^{i} \bar{B}_{i}=0 .
$$

Esta decomposição é reminiscente do teorema de Helmholtz, que garante que todo vetor pode ser escrito como a soma de um vetor de rotacional nulo $\left(\partial_{i} B\right)$ e um vetor de divergência nula $\left(\bar{B}_{i}\right)$. Notemos que a decomposição acima não é única, pois o escalar $B=\nabla^{-2}\left(\partial^{i} B_{i}\right)$ permanece inalterado se a ele adicionarmos uma função harmônica qualquer. No entanto, a unicidade da decomposição fica garantida se supusermos que os campos são tais que se anulam no infinito.

De maneira análoga, todo tensor simétrico de ordem 2 admite uma decomposição única em termos de funções escalares, vetoriais e tensoriais. Em particular, o tensor $h_{i j}$ em (3.4), pode ser decomposto univocamente na forma

$$
h_{i j}=2 C \delta_{i j}+2 \partial_{i} \partial_{j} E+2 \partial_{(i} E_{j)}+2 E_{i j}
$$

onde os fatores de 2 são introduzidos por conveniência futura. Os modos $E_{i}$ e $E_{i j}$ também estão sujeitos a certas condições de transversalidade

$$
\partial^{i} E_{i}=0=E_{i}^{i}, \quad \partial^{i} E_{i j}=0 .
$$

Novamente, condições de contorno apropriadas são necessárias para garantir a unicidade da decomposição acima [52]. De modo geral, será conveniente decompor um tensor qualquer na soma do seu traço com um tensor de traço nulo. Para o tensor $h_{i j}$, teremos

$$
h_{i j}=\frac{h}{3} \delta_{i j}+h_{i j}^{\mathrm{TN}},
$$

ou, em termos da decomposição EVT,

$$
h_{i j}=S \delta_{i j}+2 D_{i j} E+2 \partial_{(i} E_{j)}+2 E_{i j}
$$

sendo que

$$
S \equiv 2 C+\frac{2}{3} \nabla^{2} E, \quad D_{i j} \equiv \partial_{i} \partial_{j}-\frac{1}{3} \nabla^{2} \delta_{i j} .
$$


Concluindo, portanto, a decomposição EVT da métrica (3.4) nos permite reescrever seus 10 graus de liberdade iniciais em termos de quatro funções escalares $(A, B, S$ e $E$ ), dois vetores de divergência nula $\left(\bar{B}_{i}\right.$ e $\left.E_{i}\right)$ e um tensor sem traço e de divergência nula $\left(E_{i j}\right)$. Essa decomposição é única e sempre possível de ser feita em quantidades definidas sobre variedades homogêneas [52]. A grande vantagem dessa decomposição reside no fato de que, em primeira ordem na teoria de perturbação, os modos EVT das perturbações de um universo homogêneo e isotrópico se desacoplam. Dessa forma as equações dinâmicas de cada modo também se desacoplam, e suas soluções podem ser analisadas separadamente. Essa característica simplificadora não existe em universos cuja métrica de fundo é apenas homogênea. Nesses casos a anisotropia do espaço é capaz de acoplar os modos EVT mesmo em primeira ordem nas perturbações, resultando num sistema de equações dinâmicas acopladas. Trataremos este problema em detalhes na segunda parte deste trabalho.

\subsubsection{Operadores de projeção}

A decomposição EVT consiste em reescrever uma quantidade tensorial qualquer em seus modos escalares, vetoriais e tensoriais. Por outro lado, dado uma quantidade já decomposta, como podemos obter seus modos EVT isoladamente? Para um dado vetor $V_{i}$, a decomposição

$$
V_{i}=\partial_{i} V+\bar{V}_{i}, \quad \partial^{i} \bar{V}_{i}=0
$$

nos mostra que as partes escalar e vetorial podem ser formalmente extraídas com a ajuda dos operadores de projeção $\nabla^{-2} \partial^{i}$ e $\delta_{i}^{j}-\partial_{i} \nabla^{-2} \partial^{j}$. De fato, pois

$$
\left(\nabla^{-2} \partial^{i}\right) V_{i}=V, \quad\left(\delta_{i}^{j}-\partial_{i} \nabla^{-2} \partial^{j}\right) V_{j}=\bar{V}_{i} .
$$

Esses dois operadores são não-locais, pois dependem do inverso do operador de Laplace: $\nabla^{-2}$. Uma definição rigorosa dos mesmos pode ser obtida através de integrais sobre todo o espaço, definidas em termos de funções de Green apropriadas. Outra solução é definir os operadores no espaço de Fourier. Nesse caso a identificação

$$
V_{i}(\boldsymbol{x}) \rightarrow \int \mathrm{d}^{3} k V_{i}(\boldsymbol{k}) e^{\mathrm{i} \boldsymbol{k} \cdot \boldsymbol{x}}, \quad \partial_{i} \rightarrow \mathrm{i} k_{i}
$$

permite rescrever tanto a decomposição EVT como os operadores de projeção em termos dos vetores de onda $k_{i}$. No espaço de Fourier, a decomposição (3.14) se torna ${ }^{1}$

$$
V_{i}=\hat{k}_{i} V+\bar{V}_{i}, \quad \hat{k}^{i} \bar{V}_{i}=0
$$

onde $\hat{k}_{i} \equiv k_{i} / k$. Vale notar que a métrica subentendida no produto escalar $\boldsymbol{k} \cdot \boldsymbol{x}$ é a delta de Kronecker, portanto a ordem dos índices espaciais não é importante aqui. Os operadores de projeção dos modos escalares e vetoriais podem agora ser definidos por

$$
\begin{aligned}
P^{i} & \equiv \hat{k}^{i} \\
P_{j}^{i} & \equiv \delta_{j}^{i}-\hat{k}^{i} \hat{k}_{j}
\end{aligned}
$$

\footnotetext{
${ }^{1}$ Fatores extras de $k$ e de i podem sempre ser absorvidos numa redefinição dos modos EVT.
} 
de onde segue que $P^{i} V_{i}=V$ e $P_{j}^{i} V_{i}=\bar{V}_{j}$. De maneira análoga, a decomposição EVT de um tensor $T_{i j}$ no espaço de Fourier é

$$
T_{i j}=S \delta_{i j}+\left(\hat{k}_{i} \hat{k}_{j}-\frac{1}{3} \delta_{i j}\right) T+\hat{k}_{(i} \bar{T}_{j)}+\bar{T}_{i j}, \quad \hat{k}^{i} \bar{T}_{i}=0=\bar{T}_{i}^{i}, \quad \hat{k}^{i} \bar{T}_{i j}=0 .
$$

Os operadores de projeção nesse caso são definidos da seguinte maneira

$$
\begin{aligned}
Q^{i j} & \equiv \frac{3}{2}\left(\hat{k}^{i} \hat{k}^{j}-\frac{1}{3} \delta^{i j}\right) \\
Q_{l}^{i j} & \equiv P^{i j} P_{l} \\
Q_{l m}^{i j} & \equiv P_{l}^{i} P_{m}^{j}-\frac{1}{2} P^{i j} P_{l m} .
\end{aligned}
$$

Dessas definições, segue que $Q^{i j} T_{i j}=T, Q_{l}^{i j} T_{i j}=\bar{T}_{l}$ e $Q_{l m}^{i j} T_{i j}=\bar{T}_{l m}$. O traço da decomposição pode ser trivialmente extraído tomando-se $\delta^{i j} T_{i j} / 3$. Nós retomaremos esse assunto na parte 2 da tese, onde a decomposição dos modos escalares, vetoriais e tensoriais será feita sobre o espaço-tempo de Bianchi I. O procedimento de decomposição nesse caso será qualitativamente o mesmo, embora alguns cuidados devam ser tomados com a implementação da decomposição de Fourier, já que a métrica da seção espacial do universo de Bianchi também é uma função do tempo. Veremos também que a decomposição EVT é de importância crucial na dedução correta das equações dinâmicas das perturbações. Devido à complexidade do acoplamento dos modos nesse caso, uma extração ingênua dos modos pode levar a termos de massa e freqüências errados [53].

\subsection{Invariância de Calibre}

A relatividade geral é uma teoria na qual os observáveis físicos independem de quaisquer transformações contínuas do sistema de coordenadas. Tecnicamente, dizemos que a teoria é invariante por difeomorfismos. Sua implementação é feita através da linguagem de variedades diferenciáveis e de sistemas de coordenadas definidos nessas variedades. Portanto, quando consideramos a subtração de duas quantidades quaisquer $\mathcal{Q}$ e $\mathcal{Q}_{0}$ definidas em variedades diferentes, devemos levar em conta que essa subtração não é, em geral, bem definida. Consideremos por exemplo um campo de velocidades idealizado $V_{0}^{\mu}(\eta, \boldsymbol{x})$, definido em um universo homogêneo e isotrópico, e seu análogo $V^{\mu}(\eta, \boldsymbol{x})$, que representa o verdadeiro campo de velocidades do universo. Este campo pode representar, por exemplo, a distribuição espacial de velocidades do grupo local de galáxias. Tipicamente a perturbação do campo $V^{\mu}$ poderia ser definida como a diferença entre o seu valor real e o valor não-perturbado, ambos calculados num dado ponto $p$ do universo. Entretanto, na ausência de um mapeamento bem definido entre os pontos das duas variedades, essa subtração não tem sentido.

Chamemos de $\mathcal{M}_{0}$ a variedade que representa o universo homogêneo na qual, por conveniência, nosso sistema de coordenadas será mantido fixo, e de $\mathcal{M}$ a variedade que representa o universo real. Consideremos também um mapa, ou mais precisamente um difeomorfismo, $\mathcal{F}: \mathcal{M}_{0} \rightarrow \mathcal{M}$ que nos associa um ponto $p \in \mathcal{M}$ dado ao ponto $\mathcal{F}^{-1}(p) \in \mathcal{M}_{0}$. Assim podemos definir a perturbação $\delta \mathcal{Q}$ de uma quantidade $\mathcal{Q}$ qualquer, que pode ser um campo escalar, vetorial ou tensorial, da seguinte maneira

$$
\delta \mathcal{Q}=\mathcal{Q}(p)-\mathcal{Q}_{0}\left(\mathcal{F}^{-1}(p)\right)
$$


onde $\mathcal{Q}_{0}$ é uma quantidade não-perturbada. Em geral, a escolha do difeomorfismo $\mathcal{F}$ é arbitrária. Todavia, no tratamento linear das perturbações estaremos interessados apenas em difeomorfismos que induzam transformações infinitesimais do sistema de coordenadas

$$
x^{\mu} \rightarrow x^{\mu}-\varepsilon \xi^{\mu}(\eta, \boldsymbol{x}),
$$

sendo $|\varepsilon| \ll 1$. Assim sendo, para um segundo difeomorfismo $\widehat{\mathcal{F}}: \mathcal{M}_{0} \rightarrow \mathcal{M}$, teremos

$$
\widehat{\delta \mathcal{Q}}=\mathcal{Q}(p)-\mathcal{Q}_{0}\left(\widehat{\mathcal{F}}^{-1}(p)\right) .
$$

Claramente, a diferença na definição das perturbações induzida pelas escolhas dos mapas $\mathcal{F}$ e $\widehat{\mathcal{F}}$

$$
\Delta \mathcal{Q}(p) \equiv \widehat{\delta \mathcal{Q}}(p)-\delta \mathcal{Q}(p)=\widehat{\mathcal{Q}}(p)-\mathcal{Q}(p)
$$

não pode ter sentido físico, pois representa apenas um artifício introduzido pela escolha desses difeomorfismos. Isso significa que a escolha dos mapas, que nesse caso se resume à escolha da função $\xi^{\mu}$, está ligada a uma escolha de calibre da teoria. Podemos portanto definir quantidades invariantes de calibre como sendo aquelas para as quais a diferença (3.27) é nula

$$
\Delta \mathcal{Q}(p)=0 \quad \rightarrow \quad \mathcal{Q}=\text { invariante de calibre }
$$

Podemos nos perguntar agora como um campo tensorial arbitrário se comporta quando o submetemos a uma transformação do sistema de coordenadas da forma (3.25). Para tanto lembremos da lei geral de transformação de um campo tensorial

$$
\bar{T}_{\nu_{1} \ldots}^{\mu_{1} \ldots}\left(\bar{x}_{p}^{\lambda}\right)=\left.\left(\frac{\partial \bar{x}^{\mu_{1}}}{\partial x^{\sigma_{1}}} \cdots \frac{\partial x^{\rho_{1}}}{\partial \bar{x}^{\nu_{1}}} \cdots\right)\right|_{p} T_{\rho_{1} \ldots}^{\sigma_{1} \ldots}\left(x_{p}^{\lambda}\right) .
$$

onde o sub-índice $p$ indica o ponto onde essas quantidades devem ser avaliadas. Substituindo $\bar{x}^{\mu}=x^{\mu}-\varepsilon \xi^{\mu}$ na transformação acima e coletando termos lineares em $\varepsilon$, chegamos à definição da derivada de Lie

$$
\bar{T}_{\nu_{1} \ldots}^{\mu_{1} \ldots}\left(x_{p}^{\lambda}\right)=T_{\nu_{1} \ldots}^{\mu_{1} \ldots}\left(x_{p}^{\lambda}\right)+\varepsilon \mathcal{L}_{\xi} \bar{T}_{\nu_{1} \ldots}^{\mu_{1} \ldots}\left(x_{p}^{\lambda}\right)+\mathcal{O}\left(\varepsilon^{2}\right) .
$$

Assim, concluímos que a dependência de uma quantidade geométrica qualquer com a escolha de calibre é proporcional à derivada de Lie dessa quantidade com relação à função de calibre $\xi^{\mu}$

$$
\Delta \mathcal{Q} \simeq \mathcal{L}_{\xi} \mathcal{Q}_{0}
$$

onde tomamos $\varepsilon=1$ e consideramos apenas o primeiro termo da expansão $\mathcal{Q}=$ $\mathcal{Q}_{0}+\delta \mathcal{Q}$. Portanto, a condição necessária e suficiente para que a perturbação $\Delta \mathcal{Q}$ independa da escolha de calibre é

$$
\mathcal{L}_{\xi} \mathcal{Q}_{0}=0
$$

Esse resultado é conhecido como o lema de Stewart-Walker [52]. Notemos que a única maneira de satisfazer a condição acima é se $\mathcal{Q}_{0}$ for uma constante. Em outras palavras, quantidades geométricas (covariantes) são intrinsecamente dependentes do calibre. 


\subsubsection{Transformações de calibre e quantidades invariantes}

Da discussão acima podemos deduzir as leis de transformação de calibre das funções introduzidas em (3.4) e (3.6). É importante mencionar que tanto o parâmetro de calibre como as perturbações da métrica e do inflaton são considerados pequenos. Assim, na linearização das equações desprezaremos não só termos quadráticos em $\delta g_{\mu \nu}, \delta \phi$ e $\xi^{\mu}$ como também qualquer produto dessas quantidades.

\section{Setor geométrico}

Para calcular a transformação de calibre (3.31) dos componentes da métrica notemos que a derivada de Lie nesse caso será dada simplesmente por

$$
\mathcal{L}_{\xi} g_{\mu \nu}=2 \nabla\left(\mu \xi_{\nu)}\right.
$$

onde $\nabla_{\mu}$ é a derivada covariante compatível com a métrica de fundo $g_{\mu \nu}$. Decompondo a função $\xi^{\mu}$ em seus modos EVT

$$
\xi^{0}=T(\eta, \boldsymbol{x}), \quad \xi^{i}=\partial^{i} L(\eta, \boldsymbol{x})+L^{i}(\eta, \boldsymbol{x})
$$

e notando que $\xi_{\mu}=g_{\mu \nu} \xi^{\nu}$, chegamos às seguintes leis de transformação:

- Modos escalares

$$
\begin{aligned}
& A \rightarrow \widehat{A}=A+\frac{(a T)^{\prime}}{a} \\
& B \rightarrow \widehat{B}=B-T+L^{\prime} \\
& C \rightarrow \widehat{C}=C+\mathcal{H} T \\
& E \rightarrow \widehat{E}=E+L
\end{aligned}
$$

- Modos vetoriais

$$
\begin{aligned}
& \bar{B}^{i} \rightarrow \widehat{\bar{B}^{i}}=\bar{B}^{i}+L^{i \prime} \\
& E^{i} \rightarrow \widehat{E^{i}}=E^{i}+L^{i}
\end{aligned}
$$

- Modos tensoriais

$$
E_{i j} \rightarrow \widehat{E_{i j}}=E_{i j}
$$

Com exceção do modo tensorial, os modos escalares e vetoriais da métrica (3.4) não são invariantes sob uma transformação de calibre, pois todos dependem explicitamente de $\xi^{\mu}$. Isso é uma conseqüência direta do lema de Stewart-Walker: quantidades covariantes não são em geral invariantes. À primeira vista isso parece impedir a construção de uma teoria de perturbações em termos de quantidades físicas, as quais claramente não devem depender da escolha da função de calibre. No entanto, podemos sempre construir quantidades definidas com relação a um sistema particular de coordenadas, cuja expressão é válida não só num ponto mas em todos os pontos ligados a este pela transformação (3.25). Em outras palavras, tais quantidades "não-geométricas", conhecidas como variáveis de Bardeen [51], independem da escolha de $\xi^{\mu}$ e portanto são candidatas perfeitas para a descrição das variáveis físicas. Para os modos escalares e vetoriais das perturbações, as variáveis de Bardeen são: 
- Modos escalares

$$
\begin{aligned}
\Phi & \equiv A+\frac{1}{a}\left[a\left(B-E^{\prime}\right)\right]^{\prime} \\
\Psi & \equiv-C-\mathcal{H}\left(B-E^{\prime}\right)
\end{aligned}
$$

- Modos vetoriais

$$
\Phi^{i} \equiv \bar{B}^{i}-E^{i \prime}
$$

Existem infinitas outras "variáveis" de Bardeen, dado que qualquer combinação linear das funções acima também será invariante. No entanto, somente 6 dessas variáveis são linearmente independentes, pois dos 10 graus de liberdade iniciais da métrica (3.4), quatro podem ser eliminados com uma escolha apropriada da função de calibre. As variáveis acima representam portanto apenas os graus de liberdade físicos da métrica.

\section{Setor de matéria}

Sob uma transformação de calibre, a perturbação (3.6) do inflaton se transforma, em primeira ordem, como

$$
\delta \phi \rightarrow \widehat{\delta \phi}=\phi^{\prime} T+\delta \phi .
$$

A quantidade invariante associada à perturbação do inflaton pode ser definida como

$$
\chi \equiv \delta \phi+\left(B-E^{\prime}\right) \phi^{\prime} .
$$

\subsubsection{O calibre newtoniano}

Embora o procedimento de construção das variáveis de Bardeen seja sempre possível, devemos garantir que as equações das perturbações poderão ser escritas em termos dessas variáveis. De fato, as equações de Einstein sendo covariantes podem sempre ser escritas na forma $S_{\mu \nu}=G_{\mu \nu}-8 \pi G T_{\mu \nu}$. Portanto o lema (3.32) se aplica trivialmente ao tensor $S_{\mu \nu}$. Isso garante, ao menos em primeira ordem nas perturbações, que as equações de Einstein possam ser escritas univocamente em função das variáveis introduzidas acima. Na prática, contudo, é muitas vezes conveniente adotar um calibre específico.

O calibre newtoniano, ou calibre longitudinal, é definido pela seguinte escolha da função (3.34)

$$
T=B-E^{\prime}, \quad L=-E, \quad L^{i \prime}=-\bar{B}^{i} .
$$

Com essa escolha, os potenciais $E, B$ e $\bar{B}^{i}$ da métrica se transformam da seguinte maneira

$$
B \rightarrow \widehat{B}=0, \quad E \rightarrow \widehat{E}=0, \quad \bar{B}^{i} \rightarrow \widehat{B^{i}}=0
$$

e o elemento de linha do universo perturbado fica dado por

$$
\mathrm{d} s^{2}=a^{2}(\eta)\left[-(1+2 A) \mathrm{d} \eta^{2}+\left((1+2 C) \delta_{i j}+2 \partial_{(i} E_{j)}+2 E_{i j}\right) \mathrm{d} x^{i} \mathrm{~d} x^{j}\right]
$$

Outras escolhas de calibre são possíveis. No entanto o calibre newtoniano tem algumas vantagens que o tornam particularmente interessante. Em primeiro lugar, as relações (3.47) fixam completamente o calibre, de tal sorte que modos residuais ou "não-físicos" são completamente eliminados das equações. Em segundo lugar, se o lado 
esquerdo das equações de Einstein for descrito por um fluido perfeito tal que $\delta T_{j}^{i} \propto \delta_{j}^{i}$, as variáveis de Bardeen se reduzem nesse calibre ao potencial gravitacional newtoniano. Os potências $\Phi$ e $\Psi$ podem portanto ser considerados como uma generalização dos potenciais gravitacionais clássicos. Finalmente, notemos que nesse calibre as variáveis $A, C, E^{i}$ e $\delta \phi$ se reduzem às quantidades invariantes

$$
\Phi=A, \quad \Psi=-C, \quad \Phi^{i}=E^{i \prime}, \quad \chi=\delta \phi
$$

Essa propriedade peculiar sugere uma dedução bastante simples e elegante das equações de Einstein na forma invariante de calibre. Podemos inicialmente escrever as equações no calibre newtoniano, onde as variáveis da métrica se reduzem às variáveis de Bardeen. Uma vez escritas as equações podemos tomar a transformação de calibre inversa. Como vimos, o lema de Stewart-Walker garante que essas equações possam ser escritas numa forma completamente invariante, ou seja, independente de $\xi^{\mu}$, portanto os termos de calibre que surgirão na transformação inversa devem necessariamente se cancelar, deixando as equações formalmente inalteradas. Em outras palavras, as equações que resultam de um cálculo feito no calibre newtoniano são as mesmas que se obtém no cálculo geral, para isso basta fazer a identificação (3.50). Esse resultado pode ser visto como um corolário do lema de Stewart-Walker.

\subsection{Equações Linearizadas}

Temos agora todos os ingredientes necessários para escrever as equações de Einstein linearizadas para as perturbações cosmológicas. Em linhas gerais, o procedimento para a obtenção das equações é o seguinte: trabalhando no calibre newtoniano, aplicamos os operadores de projeção definidos em $§ 3.2 .1$ sobre a equação (3.3); dessa forma podemos extrair, modo a modo, as equações para as pertubações. Contudo, se a métrica de fundo for homogênea e isotrópica, os modos EVT não se acoplarão no regime linear das perturbações. Isso permite que os diferentes modos perturbativos sejam identificados "visualmente", tornando o processo formal de extração dos mesmos desnecessário. Entretanto, este é o procedimento rigoroso de extração das equações e, de fato, ele está presente em todos os cálculos de perturbações sobre universos isotrópicos, embora raramente nos demos conta disso. A razão pela qual enfatizamos a importância desse procedimento é que, sem ele, a dedução das equações perturbativas em universos anisotrópicos é praticamente impossível, como veremos na segunda parte do presente trabalho.

Para obter as equações das perturbações precisamos antes conhecer as expansões perturbativas dos tensores de Einstein e de energia-momento em função das variáveis (3.50). O procedimento de obtenção dessas quantidades é imediato, porém trabalhoso, e portanto não será detalhado aqui. No entanto, esses cálculos são amplamente conhecidos e uma descrição minuciosa de todos as quantidades envolvidas pode ser encontrada em [54]. Além disso, os resultados que serão detalhados na segunda parte desse trabalho se reduzem aos aqui omitidos no limite isotrópico do universo. Esses, por sua vez, podem ser encontrados no Apêndice B. Portanto, apresentaremos aqui apenas os resultados. Em primeira ordem nas perturbações da métrica, os componen- 
tes do tensor de Einstein são

$$
\begin{aligned}
a^{2} \delta G_{0}^{0}= & 6 \mathcal{H}^{2} \Phi+6 \mathcal{H} \Psi^{\prime}-2 \nabla^{2} \Psi \\
a^{2} \delta G_{i}^{0}= & -2 \partial_{i}\left(\Psi^{\prime}+\mathcal{H} \Phi\right)+\frac{1}{2} \nabla^{2} \Phi_{i} \\
a^{2} \delta G_{j}^{i}= & \partial^{i} \partial_{j}(\Psi-\Phi)+\delta_{j}^{i}\left[2 \Psi^{\prime \prime}+\left(2 \mathcal{H}^{2}+4 \mathcal{H}^{\prime}\right) \Phi-\nabla^{2}(\Psi-\Phi)+4 \mathcal{H} \Psi^{\prime}+2 \mathcal{H} \Phi^{\prime}\right] \\
& +\partial^{(i} \Phi_{j)}^{\prime}+2 \mathcal{H} \partial^{(i} \Phi_{j)}+E_{j}^{i \prime \prime}+2 \mathcal{H} E_{j}^{i \prime}-\nabla^{2} E_{j}^{i} .
\end{aligned}
$$

Já as perturbações do tensor de energia-momento em primeira ordem são

$$
\begin{aligned}
a^{2} \delta T_{0}^{0} & =-\phi^{\prime} \chi^{\prime}-a^{2} V,_{\phi} \chi+\Phi \phi^{\prime 2} \\
a^{2} \delta T_{i}^{0} & =-\partial_{i}\left(\phi^{\prime} \chi\right) \\
a^{2} \delta T_{j}^{i} & =-\left(\phi^{\prime 2} \Phi+a^{2} V,_{\phi} \chi-\phi^{\prime} \chi^{\prime}\right) \delta_{j}^{i} .
\end{aligned}
$$

Antes de prosseguirmos, notemos que a perturbação da parte $(i, j)$ do tensor de energia momento é diagonal: $\delta T_{j}^{i} \propto \delta_{j}^{i}$. Por outro lado, o modo escalar do tensor $\delta G_{j}^{i}$ possui uma parte não diagonal, dada por $\partial^{i} \partial_{j}(\Psi-\Phi)$. Essa parte precisa necessariamente ser nula, o que equivale ao seguinte vínculo

$$
\Phi=\Psi
$$

o qual passaremos a usar de agora em diante. Esse resultado é bem conhecido e se aplica não somente ao caso das perturbações do inflaton mas a qualquer conteúdo de matéria cujo tensor de energia-momento é o de um fluido perfeito.

\subsubsection{Modos escalares}

Existem quatro equações para os modos escalares. Três delas estão contidas nos componentes $(0,0),(0, i)$ e $(i, j)$ das equações de Einstein. Elas são respectivamente dadas por

$$
\begin{aligned}
\nabla^{2} \Phi-3 \mathcal{H}\left(\Phi^{\prime}+\mathcal{H} \Phi\right) & =\frac{\kappa}{2}\left(\phi^{\prime} \chi^{\prime}+a^{2} V,_{\phi} \chi-\Phi \phi^{2}\right) \\
\frac{(a \Phi)^{\prime}}{a} & =\frac{\kappa}{2} \phi^{\prime} \chi \\
\Phi^{\prime \prime}+3 \mathcal{H} \Phi^{\prime}+\left(\mathcal{H}^{2}+2 \mathcal{H}^{\prime}\right) \Phi & =\frac{\kappa}{2}\left(\phi^{\prime} \chi^{\prime}-\phi^{\prime 2} \Phi-a^{2} V,_{\phi} \chi\right) .
\end{aligned}
$$

A quarta equação é a equação de Klein-Gordon perturbada

$$
\chi^{\prime \prime}+2 \mathcal{H} \chi^{\prime}-\nabla^{2} \chi+a^{2} V,_{\phi \phi} \chi=2 \frac{\left(a^{2} \phi^{\prime}\right)^{\prime}}{a^{2}} \Phi+4 \phi^{\prime} \Phi^{\prime} .
$$

Dos dois graus de liberdade restantes, $\Phi$ e $\chi$, apenas um é necessário para descrever a dinâmica das perturbações escalares, pois conhecendo-se a solução do modo $\Phi$, a equação de vínculo (3.59) determina a dinâmica do modo $\chi$. Iremos, portanto, nos limitar a apenas uma das equações acima. Combinando (3.58) e (3.60) e utilizando a equação (2.35) chegamos a

$$
\Phi^{\prime \prime}+2\left(\mathcal{H}-\frac{\phi^{\prime \prime}}{\phi^{\prime}}\right) \Phi^{\prime}+\left[2\left(\mathcal{H}^{\prime}-\mathcal{H} \frac{\phi^{\prime \prime}}{\phi^{\prime}}\right)-\nabla^{2}\right] \Phi=0 .
$$


Apesar de sua aparente complexidade, essa equação admite uma primeira integral exata. Definindo uma nova variável na forma

$$
\mathcal{R} \equiv \Phi+\frac{2 \mathcal{H}}{\kappa \phi^{\prime 2}}\left(\Phi^{\prime}+\mathcal{H} \Phi\right)
$$

podemos mostrar, utilizando a equação (3.62), que

$$
\mathcal{R}^{\prime}=\frac{2 \mathcal{H}}{\kappa \phi^{\prime 2}} \nabla^{2} \Phi
$$

A variável $\mathcal{R}$ possui uma interpretação muito simples no calibre comóvel, onde ela corresponde à perturbação de curvatura da seção espacial do universo [55]. No limite de grandes comprimentos de onda essa variável se conserva, o que é facilmente verificado no espaço de Fourier

$$
\lim _{\boldsymbol{k} \rightarrow 0} \mathcal{R}^{\prime}=0
$$

A constância da variável nesse limite permite relacionar o valor do potencial gravitacional durante a inflação ao seu valor no início da era da radiação. Essa característica também é crucial para a quantização das perturbações escalares, pois garante que a amplitude dos modos seja conservada, independente da física envolvida no fim da era da inflação.

\subsubsection{Modos vetoriais}

As equações para os modos vetoriais estão contidas nos componentes $(0, i)$ e $(i, j)$ das equações de Einstein. Elas são:

$$
\begin{aligned}
\nabla^{2} \Phi_{i} & =0, \\
\Phi_{i}^{\prime}+2 \mathcal{H} \Phi_{i} & =0 .
\end{aligned}
$$

A segunda equação nos mostra que os modos vetoriais decaem rapidamente num universo em expansão

$$
\Phi_{i}(\eta, \boldsymbol{x})=C_{i}(\boldsymbol{x}) a^{-2},
$$

ou seja, esses modos precisam ter uma amplitude muito grande no começo da inflação para que hoje as perturbações vetoriais sejam detectáveis. A primeira equação impõe vínculos ainda mais fortes sobre as soluções. Funções harmônicas não admitem máximos nem mínimos, de sorte que a única solução compatível com a equação de Laplace e tal que $C_{i}(\boldsymbol{x} \rightarrow \infty)=0$ é a solução trivial

$$
C_{i}(\boldsymbol{x})=0, \quad \forall \boldsymbol{x}
$$

Mesmo nos casos em que o conteúdo de energia do universo tem um componente vetorial não-nulo, os modos vetoriais decaem com o quadrado do fator de escala, e seu impacto na dinâmica do universo pode ser desprezado. Em universos anisotrópicos os modos vetoriais também têm uma dinâmica negligenciável. Porém, nesses casos, eles surgem como vínculos entre as perturbações escalares e tensoriais e, portanto, não podem ser ignorados [1]. 


\subsubsection{Modos tensoriais}

A única contribuição para os modos tensoriais está na equação $(i, j)$ de Einstein,

$$
E_{i j}^{\prime \prime}+2 \mathcal{H} E_{i j}^{\prime}-\nabla^{2} E_{i j}=0
$$

Esta equação representa a dinâmica das ondas gravitacionais primordiais, criadas no regime inflacionário do universo. 


\section{CAPÍTULO 4}

\section{Quantização das Perturbações}

Faremos neste capítulo uma das aplicações mais importantes do paradigma inflacionário, que é a quantização das perturbações cosmológicas durante a era da inflação. Em pequenos comprimentos de onda, as perturbações escalares e tensoriais do universo primordial se comportam essencialmente como ondas planas no espaço de Minkowski. Isso nos permite fixar a amplitude das perturbações de maneira livre de ambigüidades e compatível com o princípio de incerteza de Heisenberg. Para tanto usaremos o procedimento padrão de quantização canônica da teoria de campos.

A implementação da quantização canônica depende da existência de um conjunto de variáveis canonicamente conjugadas a partir das quais, via regras de comutação, poderemos fixar a amplitude das perturbações. Será necessário portanto identificar corretamente os graus de liberdade das perturbações cosmológicas, bem como suas variáveis conjugadas de "posição" e "momento". Em outras palavras, precisamos conhecer a lagrangiana efetiva das perturbações cosmológicas.

Como vimos, tanto os modos escalares como os modos tensoriais são descritos por equações diferenciais lineares de segunda ordem. Essas duas equações podem ser reescritas na forma de equações de osciladores harmônicos paramétricos, ou seja, osciladores com uma freqüência dependente do tempo. Definindo novas variáveis $u$ e $\mu_{i j}$ através de

$$
u \equiv \frac{2}{\kappa} \frac{a \Phi}{\phi^{\prime}}, \quad \mu_{i j} \equiv a E_{i j}
$$

as equações (3.62) e (3.70) ficam determinadas respectivamente por

$$
u^{\prime \prime}-\left(\nabla^{2}+\frac{\theta^{\prime \prime}}{\theta}\right) u=0 \quad \text { e } \quad \mu_{i j}^{\prime \prime}-\left(\nabla^{2}+\frac{a^{\prime \prime}}{a}\right) \mu_{i j}=0
$$

onde $\theta \equiv \mathcal{H} / a \phi^{\prime}$. Olhando para essas equações, somos tentados a postular suas densidades lagrangianas

$$
\mathcal{L}_{u}=\frac{1}{2}\left[\left(u^{\prime}\right)^{2}-\partial_{i} u \partial^{i} u+\frac{\theta^{\prime \prime}}{\theta} u^{2}\right], \mathcal{L}_{\mu}=\frac{1}{2}\left[\left(\mu_{i j}\right)^{\prime}\left(\mu^{i j}\right)^{\prime}-\partial_{l} \mu_{i j} \partial^{l} \mu^{i j}+\frac{a^{\prime \prime}}{a} \mu_{i j} \mu^{i j}\right]
$$


o que imediatamente nos forneceria os pares $\left(u, u^{\prime}\right)$ e $\left(\mu_{i j}, \mu_{i j}^{\prime}\right)$ de variáveis canônicas. Esse procedimento funcionaria para as perturbações tensoriais, mas nos levaria a variáveis canônicas erradas para as perturbações escalares. Veremos a seguir que a variável canônica das perturbações escalares é a chamada variável de Mukhanov-Sasaki, definida como $v=z \mathcal{R}$, e não a variável $u$ introduzida acima. A maneira correta de se obter as variáveis canônicas das perturbações é expandindo a ação de Einstein-Hilbert acoplada à ação do inflaton

$$
S=\frac{1}{2 \kappa} \int \mathrm{d}^{4} x \sqrt{-g}\left(R+\mathcal{L}_{\text {inf }}\right)
$$

até segunda ordem nas variáveis perturbativas. Uma vez excluídos os vínculos e as derivadas totais da ação, temos a lagrangiana efetiva das perturbações [56, 57]. A maneira mais fácil de se realizar essa tarefa é através do formalismo ADM da relatividade geral.

\subsection{Formalismo ADM}

O formalismo $\mathrm{ADM}^{1}$ é um formalismo hamiltoniano da relatividade geral, inicialmente formulado para lidar com a questão da quantização de sistemas físicos evoluindo sobre fundos gravitacionais [58]. Em linhas gerais, esse formalismo consiste em reescrever a ação de Einstein-Hilbert numa forma canônica, de modo a separar os graus de liberdade físicos da métrica dos graus de liberdade não-físicos, que surgem para garantir a covariância geral da gravitação de Einstein. Essa separação pode ser obtida parametrizando-se a métrica do espaço-tempo da seguinte forma

$$
\mathrm{d} s^{2}=-\left(N^{2}-N_{i} N^{i}\right) \mathrm{d} t^{2}+2 N_{i} \mathrm{~d} t \mathrm{~d} x^{i}+g_{i j} \mathrm{~d} x^{i} \mathrm{~d} x^{j} .
$$

Nessa representação, $g_{i j}$ é a métrica tridimensional definida em superfícies de tempo constante. As funções $N$ e $N^{i}$ são multiplicadores de Lagrange; portanto as equações de Euler-Lagrange associadas a essas variáveis são puramente algébricas. Uma vez solucionadas essas equações podemos introduzir as soluções para $N$ e $N^{i}$ de volta na ação, simplificando dessa forma os cálculos perturbativos. No formalismo ADM a ação de Einstein-Hilbert minimamente acoplada ao inflaton toma a seguinte forma

$$
\begin{array}{r}
S=\frac{1}{2 \kappa} \int \mathrm{d} t \mathrm{~d}^{3} x \sqrt{-g}\left[N R^{(3)}+N\left(K_{i j} K^{i j}-K^{2}\right)\right. \\
-\kappa N\left(g^{i j} \partial_{i} \phi \partial_{j} \phi+2 V(\phi)\right) \\
\left.+\kappa N^{-1}\left(\dot{\phi}-N^{i} \partial_{i} \phi\right)^{2}\right],(4.6)
\end{array}
$$

sendo que $R^{(3)}$ é o escalar de Ricci construído a partir da métrica $g_{i j}$ e $K_{i j}$ é o tensor de curvatura extrínseca, definido como

$$
K_{i j} \equiv \frac{N^{-1}}{2}\left(\dot{g}_{i j}-2 \nabla_{(i} N_{j)}\right), \quad K \equiv g^{i j} K_{i j}
$$

\footnotetext{
${ }^{1}$ Acrônimo de Arnowitt, Deser e Misner.
} 
Os índices espaciais serão agora manipulados com a métrica $g_{i j}$. Variando a ação com relação à função lapso $N$ e ao vetor deslocamento $N_{i}$, encontramos os vínculos de energia e momento, respectivamente:

$$
\begin{aligned}
R^{(3)}-\left(K_{i j} K^{i j}-K^{2}\right)-2 V-\kappa g^{i j} \partial_{i} \phi \partial_{j} \phi+N^{-2} \kappa\left(\dot{\phi}-N^{i} \partial_{i} \phi\right)^{2} & =0 \\
\nabla_{j}\left(K_{i}^{j}-K \delta_{i}^{j}\right)-N^{-1} \kappa\left(\dot{\phi}-N^{j} \partial_{j} \phi\right) \partial_{i} \phi & =0 .
\end{aligned}
$$

Comparando a métrica (3.49) no calibre newtoniano com a métrica (4.5), concluímos que a função lapso e o vetor deslocamento são dados, no tempo físico, respectivamente por

$$
N^{2}=(1+2 \Phi), \quad N_{i}=0
$$

e que a métrica $g_{i j}$ é igual a

$$
g_{i j}=a^{2}\left[\delta_{i j}(1-2 \Psi)+2 \partial_{(i} E_{j)}+2 E_{i j}\right] .
$$

Introduzindo (4.10-4.11) nas equações (4.8-4.9) obtemos, em primeira ordem, expressões para os vínculos em termos das variáveis da métrica. Essas expressões são então substituídas na ação (4.6) onde, em seguida, todas as quantidades são expandidas até segunda ordem. Nesse processo as equações de fundo (2.34) e (2.35) são usadas para simplificar os cálculos e identificar derivadas totais na ação. De fato, boa parte do procedimento consiste em identificar e isolar derivadas totais. O motivo é que, assumindo que os campos se anulam no infinito espacial, essas derivadas não contribuem para a dinâmica dos campos e podem ser ignoradas. Esses são os passos gerais para a obtenção da ação quadrática. O procedimento, contudo, é bastante trabalhoso e por essa razão não apresentaremos os detalhes aqui. Alguns passos podem ser encontrados em [50]. Além disso, na parte 2 dessa tese apresentaremos os detalhes do cálculo para um universo anisotrópico onde, no limite apropriado de universo isotrópico (a ser definido na parte 2) recuperamos os resultados da teoria isotrópica. Apresentaremos aqui, portanto, apenas os resultados. Definindo duas novas variáveis através de

$$
v \equiv z \mathcal{R}, \quad z=\frac{a \phi^{\prime}}{\mathcal{H}} \quad \text { e } \quad \mu_{i j} \equiv \sqrt{\frac{M_{\mathrm{p}}^{2}}{4}} a E_{i j}
$$

e decompondo o tensor $\mu_{i j}$ em termos das polarizações $\times \mathrm{e}+$ das ondas gravitacionais $^{2}$

$$
\mu_{i j}=\sum_{\lambda=\times,+} \mu_{\lambda} \varepsilon_{i j}^{\lambda}, \quad \varepsilon_{i j}^{\lambda} \varepsilon_{\lambda^{\prime}}^{i j}=\delta_{\lambda \lambda^{\prime}}
$$

a ação quadrática que procuramos será dada por

$$
S^{(2)}=\frac{1}{2} \int \mathrm{d} \eta \mathrm{d}^{3} x\left[\left(v^{\prime}\right)^{2}-\partial_{i} v \partial^{i} v+\frac{z^{\prime \prime}}{z} v^{2}+\sum_{\lambda=\times,+}\left(\left(\mu_{\lambda}^{\prime}\right)^{2}-\partial_{l} \mu_{\lambda} \partial^{l} \mu_{\lambda}+\frac{a^{\prime \prime}}{a} \mu_{\lambda}^{2}\right)\right] .
$$

Podemos ver que, de fato, a variável canônica das perturbações escalares não é dada por (4.1), mas sim por (4.12). As variáveis (4.12) são conhecidas como variáveis de

\footnotetext{
${ }^{2}$ Rigorosamente, a decomposição do tensor $\mu_{i j}$ em uma base de polarização deve ser implementada no espaço de Fourier. Os resultados dessa seção, contudo, independem desses detalhes.
} 
Mukhanov-Sasaki. As equações canônicas de movimento no espaço de Fourier são

$$
\begin{aligned}
v_{k}^{\prime \prime}+\left(k^{2}-\frac{z^{\prime \prime}}{z}\right) v_{k} & =0 \\
\mu_{\lambda, k}^{\prime \prime}+\left(k^{2}-\frac{a^{\prime \prime}}{a}\right) \mu_{\lambda, k} & =0 .
\end{aligned}
$$

Uma característica importante dessas equações é que elas são completamente independentes (desacopladas). Esse desacoplamento se manifesta não só na dinâmica das equações mas também nas estatísticas das perturbações primordiais.

\subsection{Procedimento Geral de Quantização}

Os setores escalar e tensorial da ação (4.14) são formalmente idênticos. A diferença está no "termo de massa" que acompanha cada modo. Em primeira ordem nos parâmetros de rolagem lenta $\epsilon$ e $\delta$, esses termos são

$$
\frac{z^{\prime \prime}}{z}=\frac{1}{\eta^{2}}(2+6 \epsilon-3 \delta), \quad \frac{a^{\prime \prime}}{a}=\frac{1}{\eta^{2}}(2+3 \epsilon) .
$$

Portanto o procedimento de quantização é essencialmente o mesmo para os dois modos. Assim sendo, apresentaremos nessa seção o procedimento geral de quantização de uma variável generalizada $Q_{\ell}(\eta, \boldsymbol{x})$, onde a índice $\ell$ representa simbolicamente os modos $v, \mu_{\times}$e $\mu_{+}$. Os resultados poderão ser aplicados ao final para cada modo particular simplesmente considerando os termos de massa efetivos (4.17). Consideremos então a ação canônica

$$
S\left[Q_{\ell}\right]=\frac{1}{2} \int \mathrm{d} \eta \mathrm{d}^{3} x\left[\left(Q_{\ell}^{\prime}\right)^{2}-\partial_{i} Q_{\ell} \partial^{i} Q_{\ell}+\frac{Z_{\ell}^{\prime \prime}}{Z_{\ell}} Q_{\ell}^{2}\right]
$$

onde o termo de massa é, em geral, uma função do tempo, e tal que $Z_{\ell}=z$ no caso escalar e $Z_{\ell}=a$ no caso tensorial. A variável de momento canonicamente conjugada ao campo $Q_{\ell}$ é

$$
\Pi_{\ell} \equiv \frac{\delta S}{\delta Q_{\ell}^{\prime}}=Q_{\ell}^{\prime}
$$

O primeiro passo na quantização desse sistema é promover essas variáveis clássicas a operadores quânticos

$$
Q_{\ell}(\eta, \boldsymbol{x}) \rightarrow \hat{Q}_{\ell}(\eta, \boldsymbol{x}), \quad \Pi_{\ell}(\eta, \boldsymbol{x}) \rightarrow \hat{\Pi}_{\ell}(\eta, \boldsymbol{x})
$$

sobre os quais impomos as seguintes regras de comutação em tempos iguais

$\left[\hat{Q}_{\ell}(\eta, \boldsymbol{x}), \hat{\Pi}_{\ell^{\prime}}(\eta, \boldsymbol{y})\right]=\mathrm{i} \delta_{\ell \ell^{\prime}} \delta^{(3)}(\boldsymbol{x}-\boldsymbol{y}), \quad\left[\hat{Q}_{\ell}(\eta, \boldsymbol{x}), \hat{Q}_{\ell^{\prime}}(\eta, \boldsymbol{y})\right]=0=\left[\hat{\Pi}_{\ell}(\eta, \boldsymbol{x}), \hat{\Pi}_{\ell^{\prime}}(\eta, \boldsymbol{y})\right]$.

Para lidar com a dependência espacial dos campos trabalharemos no espaço de Fourier, onde os operadores quânticos são decompostos na forma

$$
\begin{aligned}
& \hat{Q}_{\ell}(\eta, \boldsymbol{x})=\int \frac{\mathrm{d}^{3} k}{(2 \pi)^{3 / 2}}\left(Q_{k, \ell}(\eta) \hat{a}_{\boldsymbol{k}, \ell}+Q_{k, \ell}^{*}(\eta) \hat{a}_{-\boldsymbol{k}, \ell}^{\dagger}\right) e^{\mathrm{i} \boldsymbol{k} \cdot \boldsymbol{x}} \\
& \hat{\Pi}_{\ell}(\eta, \boldsymbol{x})=\int \frac{\mathrm{d}^{3} k}{(2 \pi)^{3 / 2}}\left(\Pi_{k, \ell}(\eta) \hat{a}_{\boldsymbol{k}, \ell}+\Pi_{k, \ell}^{*}(\eta) \hat{a}_{-\boldsymbol{k}, \ell}^{\dagger}\right) e^{\mathrm{i} \boldsymbol{k} \cdot \boldsymbol{x}}
\end{aligned}
$$


e onde $\hat{a}_{\boldsymbol{k}, \ell}$ e $\hat{a}_{\boldsymbol{k}, \ell}^{\dagger}$ são os operadores de criação e aniquilação, sujeitos às regras de comutação

$$
\left[\hat{a}_{\boldsymbol{k}, \ell}, \hat{a}_{\boldsymbol{q}, \ell^{\prime}}^{\dagger}\right]=\delta_{\ell \ell^{\prime}} \delta^{(3)}(\boldsymbol{k}-\boldsymbol{q}), \quad\left[\hat{a}_{\boldsymbol{k}, \ell}, \hat{a}_{\boldsymbol{q}, \ell^{\prime}}\right]=0=\left[\hat{a}_{\boldsymbol{k}, \ell}^{\dagger}, \hat{a}_{\boldsymbol{q}, \ell^{\prime}}^{\dagger}\right]
$$

Esses operadores são usados para construir o espaço de Fock e são formalmente definidos através do estado de vácuo $|0\rangle$ da teoria

$$
\hat{a}_{\boldsymbol{k}, \ell}|0\rangle=0, \quad \hat{a}_{\boldsymbol{k}, \ell}^{\dagger}|0\rangle=|\boldsymbol{k}, \ell\rangle, \quad \forall \boldsymbol{k} \in \mathbb{R}^{3}
$$

onde o vetor $|\boldsymbol{k}, \ell\rangle$ representa um estado arbitrário de uma partícula $\ell$ com momento $\boldsymbol{k}$. Juntas, as regras de comutação nos espaços real e de Fourier implicam que os modos clássicos devam obedecer, em qualquer instante, a relação

$$
Q_{k, \ell} \Pi_{k, \ell}^{*}-Q_{k, \ell}^{*} \Pi_{k, \ell}=i
$$

que nada mais é que o Wronskiano das soluções clássicas. Isso nos permite fixar a amplitude do modo $Q_{k, \ell}(\eta)$ de maneira unívoca e compatível com o princípio de incerteza de Heisenberg.

A discussão feita até aqui é completamente geral e sua implementação depende apenas da existência de um estado de vácuo bem definido. Como se sabe, a definição desse estado em espaços curvos arbitrários nem sempre é possível. No entanto, qualquer espaço curvo deve se assemelhar, localmente, ao espaço de Minkowski, onde o estado $|0\rangle$ definido em (4.25) existe e é único. Além disso, extrapolando a expansão do universo no sentido decrescente do tempo, é razoável supor que os modos que hoje têm comprimentos de onda arbitrariamente grandes tiveram, em algum instante no infinito passado, um comprimento de onda muito pequeno. Nesse instante a amplitude dos modos é essencialmente a mesma de um modo quântico no espaço plano. Portanto a quantização das perturbações cosmológicas será possível se e somente se tal estado de vácuo existir em algum intervalo de tempo do passado. Por outro lado, esse estado vai existir se a equação clássica de movimento

$$
Q_{k, \ell}^{\prime \prime}+\omega_{\ell}^{2}(k, \eta) Q_{k, \ell}=0, \quad \omega_{\ell}^{2}(k, \eta)=k^{2}-\frac{Z_{\ell}^{\prime \prime}}{Z_{\ell}}
$$

admitir uma solução de onda plana para algum intervalo de tempo $\Delta \eta$. Ou seja, se a solução

$$
Q_{k, \ell}(\eta) \rightarrow \frac{1}{\sqrt{2 \omega_{\ell}(k, \eta)}} e^{i \int^{\eta} \omega_{\ell}\left(k, \eta^{\prime}\right) \mathrm{d} \eta^{\prime}}
$$

satisfizer a equação (4.27) nesse intervalo. Essa solução certamente satisfaz a equação seguinte:

$$
Q_{k, \ell}^{\prime \prime}+\left(\omega_{\ell}^{2}+\Theta\right) Q_{k, \ell}=0, \quad \Theta \equiv \frac{3}{4}\left(\frac{\omega_{\ell}^{\prime}}{\omega_{\ell}}\right)^{2}-\frac{1}{2} \frac{\omega_{\ell}^{\prime \prime}}{\omega_{\ell}} .
$$

É evidente então que se a condição

$$
\left|\frac{\Theta}{\omega_{\ell}^{2}}\right| \ll 1
$$


for satisfeita para algum intervalo finito de tempo, (4.28) será solução da equação (4.27), o que significa que podemos definir um estado de vácuo, pelo menos durante o intervalo $\Delta \eta$. Essa condição é essencialmente a aproximação WKB da solução (4.28). Para freqüências da forma

$$
\omega^{2}(k, \eta)=k^{2}-\frac{\alpha^{2}}{\eta^{2}}
$$

onde $\alpha$ é um número real e aproximadamente constante, a condição WKB fica

$$
\left|\frac{\Theta}{\omega^{2}}\right|=\frac{1}{4 \alpha^{2}}\left|\frac{1-6(k \eta / \alpha)^{2}}{\left(1-(k \eta / \alpha)^{2}\right)^{3}}\right| .
$$

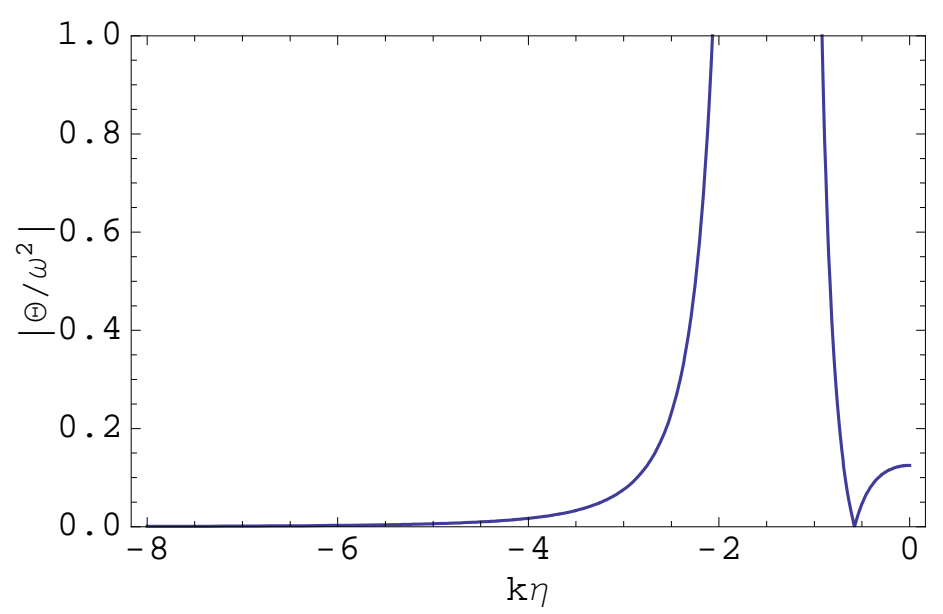

Figura 4.1: Condição WKB em função de $k \eta$ para $\alpha^{2}=2$. No limite em que $k \eta \rightarrow-\infty$ a condição WKB é satisfeita e um estado de vácuo pode ser definido.

No limite em que $k \eta \rightarrow-\infty$ a condição (4.30) é certamente satisfeita, o que confirma nossa intuição de que um estado de vácuo do tipo Minkowski existe no infinito passado do universo. Esse estado é conhecido na literatura como vácuo de BunchDavies [59] e é definido em termos da solução exata da equação (4.27) para um universo de de Sitter, onde a freqüência em (4.27) é dada exatamente por $\omega^{2}=k^{2}-2 / \eta^{2}$, ou seja

$$
Q_{k, \ell}(\eta)=\frac{1}{\sqrt{2 k}}\left(1-\frac{i}{k \eta}\right) e^{-i k \eta} \cdot \forall \ell
$$

Nos modelos inflacionários efetivos, onde o inflaton se acopla à gravidade, a expansão do universo é aproximadamente exponencial e a freqüência dos modos é dada aproximadamente por

$$
\omega_{\ell}^{2}=k^{2}-\frac{\nu_{\ell}^{2}-1 / 4}{\eta^{2}}
$$

sendo que

$$
\nu_{\ell}= \begin{cases}3 / 2+2 \epsilon-\delta & \mathrm{p} / \text { modos escalares } \\ 3 / 2+\epsilon & \mathrm{p} / \text { modos tensoriais }\end{cases}
$$


Nesses casos a equação (4.27) admite uma solução exata em termos da Função de Hankel de primeira espécie

$$
Q_{k, \ell}(\eta)=\frac{\sqrt{-\pi \eta}}{2} e^{\mathrm{i}\left(\nu+\frac{1}{2}\right) \frac{\pi}{2}} H_{\nu}^{(1)}(-k \eta)
$$

onde a amplitude já foi fixada através da condição (4.26). Essa solução é a mesma encontrada para o modelo protótipo da seção §2.3. Naquele caso a expansão quaseexponencial era uma conseqüência do termo de massa do inflaton, enquanto que nesse caso ela se deve ao acoplamento do inflaton com a gravidade, implícito na definição dos parâmetros de rolagem lenta.

Independentemente de qual seja a solução para os modos clássicos, a função de dois pontos do campo $Q_{\ell}$

$$
\left\langle 0\left|\hat{Q}_{\ell}(\eta, \boldsymbol{x}) \hat{Q}_{\ell}\left(\eta^{\prime}, \boldsymbol{y}\right)\right| 0\right\rangle \equiv\left\langle\hat{Q}_{\ell}(\eta, \boldsymbol{x}) \hat{Q}_{\ell}\left(\eta^{\prime}, \boldsymbol{y}\right)\right\rangle
$$

nos permite definir o espectro de potência das perturbações a partir da função de Green no espaço de Fourier

$$
\left\langle\hat{Q}_{\ell}(\eta, \boldsymbol{x}) \hat{Q}_{\ell}\left(\eta^{\prime}, \boldsymbol{y}\right)\right\rangle=\int \frac{\mathrm{d}^{3} k}{(2 \pi)^{3}} G_{\ell}\left(k, \eta, \eta^{\prime}\right) e^{\mathrm{i} \boldsymbol{k} \cdot \boldsymbol{x}}
$$

sendo

$$
G_{\ell}\left(k, \eta, \eta^{\prime}\right)=Q_{k, \ell}(\eta) Q_{k, \ell}^{*}\left(\eta^{\prime}\right) .
$$

O espectro de potências é então definido como o limite da função de Green no instante $\eta \rightarrow \eta^{*}$ em que os modos cruzam o raio de Hubble. Usando a definição introduzida em (2.67), temos que

$$
\mathcal{P}_{Q_{\ell}}=\left.\frac{k^{3}}{2 \pi^{2}}\left|Q_{k, \ell}(\eta)\right|^{2}\right|_{\eta=\eta^{*}},
$$

Essa expressão se aplicada igualmente aos casos escalar $\left(Q_{\ell}=v\right)$ e tensorial $\left(Q_{\ell}=\mu_{\lambda}\right)$.

\subsubsection{Perturbações de densidade}

O espectro de potência das perturbações escalares é geralmente calculado em termos da variável $\mathcal{R}=v / z$

$$
\mathcal{P}_{\mathcal{R}}=\frac{k^{3}}{2 \pi^{2}}\left|\frac{v_{k}(\eta)}{z}\right|^{2},
$$

pois ela está diretamente relacionada à perturbação do potencial gravitacional. Uma vez que $\mathcal{R}$ se conserva para perturbações com vetores de onda $k \gg a H$, as perturbações que hoje reentram o horizonte de partículas são, por hipótese, as mesmas que cruzaram o raio de Hubble durante a inflação num dado instante $\eta^{*}$. Em primeira ordem nos parâmetros $\epsilon$ e $\delta$, o espectro nesse limite vale

$$
\lim _{\frac{k}{a H} \rightarrow \infty} \mathcal{P}_{\mathcal{R}}=\left.\frac{1}{8 \pi^{2}} \frac{H^{2}(\eta)}{M_{\mathrm{p}}^{2} \epsilon}\left(\frac{k}{a H}\right)^{2 \delta-4 \epsilon}\right|_{\eta=\eta^{*}}
$$


onde enfatizamos que devido ao caráter "quase-exponencial" da expansão nesse caso, o parâmetro de Hubble adquire uma pequena dependência em $\eta$ (veja a expressão (2.39)).

Esse resultado pode ser diretamente comparado às observações. Para isso é comum parametrizá-lo por uma função do tipo $k^{n_{s}-1}$, onde $n_{s}$ é o índice espectral escalar

$$
n_{s} \equiv \frac{\mathrm{d} \ln \mathcal{P}_{\mathcal{R}}}{\mathrm{d} \ln k}=1-2 \delta-4 \epsilon .
$$

Durante a inflação $\epsilon, \delta \ll 1$ e portanto $n_{s} \lesssim 1$. Isso significa que as perturbações escalares têm uma leve preferência para a banda vermelha do espectro.

\subsubsection{Ondas gravitacionais}

De maneira análoga, o espectro de potência associado às perturbações tensoriais é dado por

$$
\mathcal{P}_{E}=\frac{k^{3}}{2 \pi^{2}} \frac{4}{M_{\mathrm{p}}^{2}} \sum_{\lambda=\times,+}\left|\frac{\mu_{\lambda}(\eta)}{a}\right|^{2} .
$$

No limite de grandes comprimentos de onda, a equação clássica (4.27) para os modos tensoriais tem uma solução crescente da forma $\mu_{\lambda} \propto a$, portanto o modo tensorial $E_{\lambda} \propto \mu_{\lambda} / a$ também se conversa nesse limite. Isso nos leva a

$$
\lim _{\frac{k}{a H} \rightarrow \infty} \mathcal{P}_{E}=\left.\frac{2}{\pi^{2}} \frac{H^{2}(\eta)}{M_{\mathrm{p}}^{2}}\left(\frac{k}{a H}\right)^{-2 \epsilon}\right|_{\eta=\eta^{*}} .
$$

Diferentemente do caso escalar, as observações nesse caso são comumente parametrizadas pela função $k^{n_{\mathrm{T}}}$, onde o indice espectral tensorial $n_{\mathrm{T}} \mathrm{é}$

$$
n_{\mathrm{T}}=-2 \epsilon .
$$

\subsubsection{Relação de consistência}

Em ambos os casos discutidos acima, as amplitudes dos espectros de potência dependem do valor de $H^{2}$ durante a inflação. Esse número, por sua vez, depende da escala de energia inflacionária imposta por alguma teoria fundamental de unificação. Entretanto, qualquer que seja essa teoria, a razão entre as amplitudes de (4.42) e (4.45) devem obedecer à seguinte relação de consistência

$$
r \equiv \frac{1}{2} \frac{\mathcal{P}_{E}}{\mathcal{P}_{\mathcal{R}}}=8 \epsilon .
$$

Essa é uma previsão bastante robusta dos modelos inflacionários construídos com um único campo escalar. De fato, para qualquer modelo da forma $V(\phi) \propto \phi^{\alpha}$, com $\alpha$ positivo, valem as relações

$$
n_{s}=1-\frac{\alpha+2}{N}, \quad r=\frac{4 \alpha}{N} .
$$

No modelo de inflação caótica $(\alpha=2)$, uma expansão de 70 e-folds requer que esses números sejam da ordem de 0.94 e 0.11, respectivamente. Esses valores são compatíveis com as observações. Os dados do quinto ano de atuação do satélite WMAP, 
tomados em conjunto com dados da medida da oscilação de bárions e de supernovas do tipo Ia, mostram que $n_{s}=0.96_{-0.013}^{+0.014}$ (68\% de confiança estatística) e $r<0.2(95 \%$ de confiança estatística) [9].

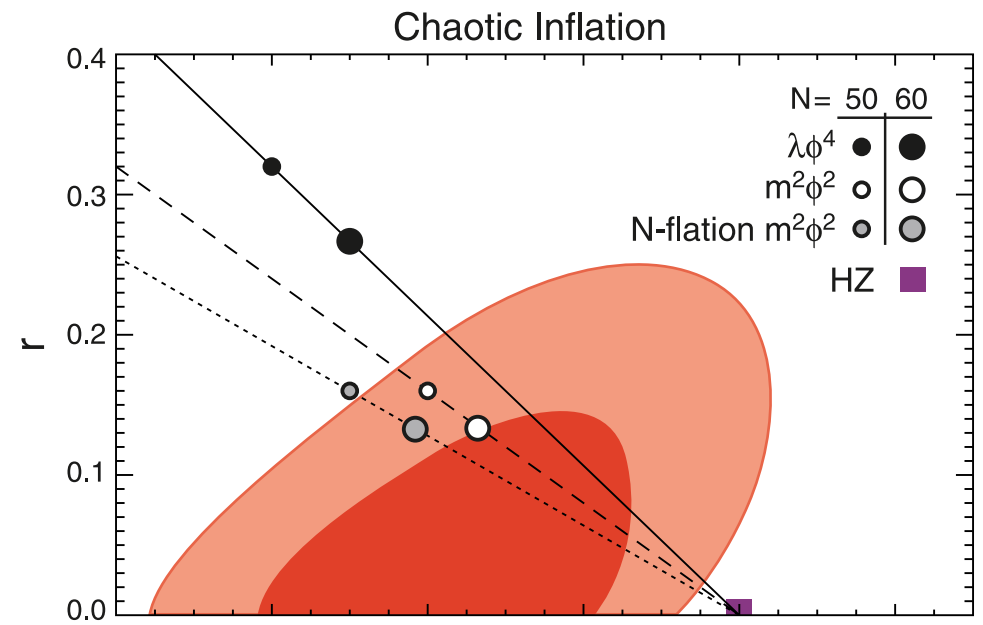

Figura 4.2: Vínculos observacionais do satélite WMAP sobre o espaço de parâmetros $r \times n_{s}$, para modelos $V(\phi) \propto \phi^{\alpha} \operatorname{com} \alpha=2$ (linha tracejada) e $\alpha=4$ (linha sólida). O modelo invariante de escala $\left(n_{s}=1\right)$ é representado pelo pequeno retângulo na base das curvas $(r=0)$. As elipses representam regiões com $68 \%$ e $95 \%$ de confiança estatística. Figura retirada de [9].

\subsection{Conclusões}

Apresentamos aqui uma revisão dos principais aspectos da teoria inflacionária, usando para isso o modelo de inflação caótica definido pelo potencial $V(\phi)=m^{2} \phi^{2} / 2$. Vimos que a teoria inflacionária, inicialmente proposta para resolver problemas relativos às condições iniciais do universo, acabou se tornando o principal modelo para a origem microscópica das estruturas de grandes escalas do universo. As previsões feitas pela teoria são robustas e podem facilmente ser reproduzidas para uma grande variedade de "modelos caóticos" de um único campo escalar. Em geral, modelos inflacionários de um único campo são capazes de prever:

1. Um universo homogêneo e isotrópico, em pleno acordo com o princípio cosmológico. Em particular, os modelos prevêem um universo que é espacialmente plano e originário de uma única região causalmente conexa.

2. Perturbações primordiais de densidade e ondas gravitacionais primordiais. Além disso, nenhuma perturbação cosmológica vetorial pode ser de origem primordial.

3. Perturbações adiabáticas, com distribuição de probabilidades gaussianas e com espectro aproximadamente invariante de escala.

4. Perturbações não-gaussianas são previstas com uma amplitude extremamente pequena em modelos de um único campo, mas podem ser obtidas com amplitudes apreciáveis em modelos com dois ou mais campos. 
Embora a robustez dos modelos seja importante para garantir à inflação o status de teoria física, ela também se traduz como uma enorme degenerescência do ponto de vista da reconstrução de modelos a partir das observações. Nesse sentido a detecção de um sinal significativo de não-gaussianidade, ou mesmo um desvio apreciável da relação de consistência seria extremamente importante para estreitar o espaço de modelos possíveis.

Duas questões importantes não foram abordadas neste trabalho. A primeira delas diz respeito à transição ao limite clássico das perturbações quânticas primordiais. Se por um lado as perturbações são geradas quanticamente, por outro elas são observadas como variáveis clássicas. Deve existir portanto um mecanismo de decoerência quântica que garanta essa transição às perturbações em algum momento da evolução do universo. Uma revisão recente sobre esses aspectos da inflação pode ser encontrada em [60]. A segunda questão omitida neste trabalho diz respeito ao fim da inflação. Em teoria, ao final do regime de rolagem lenta o inflaton oscila no fundo do seu potencial, produzindo partículas e reaquecendo o universo (veja a Fig. 2.3). Porém, na prática, um modelo efetivo de reaquecimento do universo depende, novamente, de uma teoria fundamental das partículas elementares. Na ausência dessa teoria ou de qualquer pista do modelo fundamental de unificação, fica difícil escolher um dentre tantos modelos para entender a física desse processo. Até o presente momento essa questão permanece sem resposta. 


\section{Parte II}

\section{Teoria Anisotrópica}





\section{Introdução}

A teoria do universo inflacionário é hoje um pilar da cosmologia moderna. Além de resolver os problemas introduzidos pelo modelo do Big Bang, essa teoria fornece um paradigma para a origem das estruturas de grandes escalas do universo. Em sua formulação mais simples, a inflação é capaz de fazer previsões bem definidas, tais como a existência de perturbações de densidade e de ondas gravitacionais primordiais, ambas descritas por uma estatística gaussiana e com um espectro de potência aproximadamente invariante de escala $[42,50]$. Dentro do paradigma inflacionário, a origem dessas perturbações está relacionada à amplificação das flutuações quânticas do vácuo associadas a um campo escalar - o inflaton - durante o período de expansão inflacionária do universo. Quando, por hipótese, esse período se dá num universo do tipo FriedmannRobertson-Walker (FRW), a identificação dos graus de liberdade a serem quantizados levam às conhecidas variáveis de Mukhanov-Sasaki [56, 61] introduzidas em §4.1. Isso significa assumir um universo homogêneo, isotrópico e de curvatura espacial plana em todos os cálculos, principalmente naqueles que envolvem a quantização das perturbações. Essas hipóteses de simetria se justificam a posteriori pois se o período inflacionário for longo o suficiente, todo tipo de inomogeneidades clássicas, tais como curvatura e anisotropias espaciais, serão apagadas pela expansão acelerada do universo. De fato, essa é uma das principais funções das teorias inflacionárias. Portanto, as hipóteses de homogeneidade e isotropia são perfeitamente justificáveis quando estamos interessados, por exemplo, em calcular o espectro de potência das perturbações em escalas observáveis [50]. É isso o que ocorre, por exemplo, nos modelos de inflação caótica discutido em $\S 2.2 .3$.

Embora o desenvolvimento do modelo inflacionário sobre um universo simétrico seja, por si só, de grande importância, é um fato bem conhecido que mesmo um pequeno desvio de planura $[62,63]$ ou isotropia $[64,65]$ pode ter um impacto na dinâmica do inflaton. Seria, portanto, mais satisfatório e completo do ponto de vista teórico considerar um modelo inflacionário em um espaço-tempo arbitrário e entender: (1) as condições sob as quais este universo tenderá a um universo do tipo FRW e (2) quais os efeitos de um espaço-tempo genérico na evolução e quantização das perturbações. A primeira destas questões foi abordada considerando-se o início do período inflacionário em universos inomogêneos e esfericamente simétricos em [66, 67, 68] por meio de uma análise numérica e, em [69], através de métodos semi-analíticos. O processo de isotro- 
pização do universo também já foi investigado no contexto de espaços-tempo do tipo Bianchi $[70,71,72]$ e em cenários mais exóticos envolvendo branas [73, 74, 75]. Porém, nenhum estudo se focou na segunda questão a respeito da evolução e da quantização das perturbações cosmológicas num cenário construído sobre um espaço-tempo mais genérico ${ }^{3}$. Portanto, do ponto de vista teórico, uma análise dessa natureza esclareceria ainda mais o processo padrão de quantização em espaços-tempo do tipo FRW. Já no que diz respeito às observações, debates recentes acerca de possíveis anomalias em grandes escalas angulares nos dados da Radiação Cósmica de Fundo (RCF) têm motivado inúmeros estudos. Entre essas anomalias, podemos destacar a baixa potência dos menores multipolos do espectro de temperatura RCF, o alinhamento desses mesmos multipolos e também uma assimetria entre os dois hemisférios galácticos [14, 77, 78]. Estas duas últimas sugerem um desvio de isotropia estatística no campo de temperatura da RCF e estão entre as anomalias mais evidentes. Várias explicações para essas anomalias foram propostas, tais como a quebra de isotropia devido a múltiplos campos escalares [79], existência de uma direção primordial preferencial [80], topologias exóticas do universo $[21,81,82]$ e também possíveis erros sistemáticos de foregrounds $[18,19,83]$.

A quebra de isotropia estatística das flutuações de temperatura da RCF também pode estar relacionada a uma violação local de isotropia espacial e, portanto, a um desvio de um universo do tipo FRW. Tal desvio pode ser conseqüência do período tardio de evolução do universo [84, 85] ou da dinâmica primordial num espaço-tempo anisotrópico, a qual implicaria a quebra de isotropia estatística através das condições iniciais do modelo inflacionário. Esta última hipótese foi recentemente utilizada no contexto de um universo inflacionário do tipo Bianchi I com simetria cilíndrica [80]. Nesses modelos, o componente de cisalhamento do espaço, decorrente da anisotropia do mesmo, decai com a terceira potência do fator de escala do universo. Dessa forma a anisotropia do espaço ficará impressa apenas nos primeiros modos a cruzar o horizonte de Hubble, ou seja, nos modos que correspondem aos maiores comprimentos de onda hoje observados. Além disso, uma vez que o cisalhamento do espaço decai com o tempo, a isotropização do universo é atingida assintoticamente durante a inflação. Portanto todo o período pós-inflacionário é bem descrito por um universo do tipo FRW. Conseqüentemente, os vínculos observacionais impostos pelos dados da RCF e da nucleossíntese primordial seguramente serão satisfeitos nos modelos anisotrópicos $[86,87,88]$.

Motivados por esse problemas, construiremos no presente trabalho a teoria geral das perturbações cosmológicas sobre um espaço-tempo anisotrópico do tipo Bianchi I. Tais espaços-tempo correspondem a universos espacialmente homogêneos e, portanto, são de grande importância em cosmologia. Por isso apresentamos em detalhes, em §5, um estudo semi-analítico do comportamento geral, não-perturbativo, de um universo anisotrópico e inflacionário, mostrando seus aspectos gerais e enfatizando as principais diferenças com o caso isotrópico. No que diz respeito ao estudo das perturbações cosmológicas em universos do tipo Bianchi I a principal referência encontra-se em [53]. Este trabalho, no entanto, além de conter erros conceituais e algébricos, não realiza a difícil tarefa de encontrar os graus de liberdade efetivos do sistema. Portanto, em $\S 6$,

\footnotetext{
${ }^{3}$ Embora a quantização de campos testes e o fenômeno de produção de partículas em universos anisotrópicos tenham sido abordados em [76].
} 
partindo de uma parametrização geral do espaço-tempo perturbado à la Bardeen [51], definiremos uma decomposição escalar-vetorial-tensorial (EVT) e construiremos um conjunto completo de variáveis invariantes de calibre, capazes de representar os graus de liberdade físicos do sistema. Em seguida, utilizaremos essas variáveis para construir equações dinâmicas para as perturbações cosmológicas. Após uma análise cuidadosa dos modos vetoriais, mostraremos como essas equações podem ser reduzidas a um sistema de três equações diferenciais acopladas envolvendo apenas os graus de liberdade físicos. Esses, por sua vez, correspondem à generalização anisotrópica das variáveis de Mukhanov-Sasaki. Ainda em $\S 6$ mostraremos como as equações das perturbações podem ser obtidas através de uma ação canônica envolvendo os graus de liberdade do sistema. Esse último passo é imprescindível para a quantização das perturbações cosmológicas que apresentaremos em $\S 7$. No capítulo 7 abordaremos também uma questão fundamental associada ao procedimento de quantização das perturbações cosmológicas, ou seja, a questão da definição das condições iniciais. Para resolver esse problema vamos propor um procedimento que generaliza o procedimento padrão de quantização em universos isotrópicos, mas que também leva em conta que o regime WKB, essencial para a definição de um estado de vácuo adiabático [59], nem sempre existe em universos anisotrópicos. Como conseqüência de tal fenômeno, mostraremos que as amplitudes dos modos perturbativos que violam a aproximação WKB permanecem desconhecidas e que a inflação nesse regime perde seu poder preditivo. Veremos no entanto que sempre existirá uma pequena janela de tempo onde a aproximação WKB é boa e onde o cisalhamento primordial do universo é, embora pequeno, apreciável. Finalmente, após a definição do procedimento de quantização adotado, discutiremos algumas características gerais dos espectros anisotrópicos. Em seguida, apresentaremos os resultados (numéricos) dos espectros de potências associados às perturbações cosmológicas e uma conclusão crítica dos resultados encontrados. 


\section{CAPÍTULO 5}

\section{Dinâmica Homogênea}

Os modelos mais simples de universos anisotrópicos correspondem aos espaços-tempo do tipo Bianchi I. Tais espaços pertencem ao grupo de isometrias transitivas em hipersuperfícies de tempo constante [89]. Tratam-se, portanto, de espaços-tempo com hipersuperfícies euclidianas de homogeneidade. Isso significa que o tempo é a única variável dinâmica e, conseqüentemente, as equações de Einstein reduzem-se à equações diferenciais ordinárias.

\subsection{Forma geral da métrica}

Em coordenadas comóveis e no tempo cósmico $t$ (definido em $§ 1.1$ ), a métrica dos universos de Bianchi I tem a seguinte forma

$$
\mathrm{d} s^{2}=-\mathrm{d} t^{2}+X_{1}^{2}(t)\left(\mathrm{d} x^{1}\right)^{2}+X_{2}^{2}(t)\left(\mathrm{d} x^{2}\right)^{2}+X_{3}^{2}(t)\left(\mathrm{d} x^{3}\right)^{2},
$$

onde estão incluídos três fatores de escala, a priori, diferentes. Essa métrica contém o bem conhecido espaço-tempo de FRW como um caso particular - o qual é obtido quando os três fatores de escala são iguais - e o caso extensivamente estudado de universos com simetria planar (onde apenas dois fatores de escala são diferentes). O fator de escala médio, definido por

$$
S(t) \equiv\left[X_{1}(t) X_{2}(t) X_{3}(t)\right]^{1 / 3}
$$

caracteriza a expansão do volume do universo. A partir dessa definição, a métrica (5.1) pode ser reescrita na forma

$$
\mathrm{d} s^{2}=-\mathrm{d} t^{2}+S^{2}(t) \gamma_{i j}(t) \mathrm{d} x^{i} \mathrm{~d} x^{j}
$$

onde a "métrica espacial", $\gamma_{i j}$, é a métrica definida em hipersuperfícies de tempo constante e pode ser decomposta na forma

$$
\gamma_{i j}=\exp \left[2 \beta_{i}(t)\right] \delta_{i j}
$$


sendo que as funções $\beta_{i}$ estão vinculadas por meio da relação

$$
\sum_{i=1}^{3} \beta_{i}=0
$$

Definamos agora algumas quantidades úteis como, por exemplo, os fatores de escala direcionais

$$
a_{i}(t) \equiv e^{\beta_{i}(t)}, \quad X_{i}(t)=S a_{i}(t)
$$

aos quais associaremos os seguintes parâmetros de Hubble

$$
H \equiv \frac{\dot{S}}{S}, \quad h_{i}=\frac{\dot{X}_{i}}{X_{i}}, \quad \dot{\beta}_{i}=\frac{\dot{a}_{i}}{a_{i}} .
$$

Estes, por consistência, se relacionam entre si através de

$$
h_{i}=H+\dot{\beta}_{i}, \quad H=\frac{1}{3} \sum_{i=1}^{3} h_{i},
$$

onde o ponto define a derivada com relação ao tempo físico. Vamos introduzir também algumas quantidades associadas ao fenômeno de cisalhamento do espaço. Por definição, temos (veja [1] para uma relação desta definição com o formalismo $3+1$ )

$$
\hat{\sigma}_{i j} \equiv \frac{1}{2} \dot{\gamma}_{i j}, \quad \hat{\sigma}^{2} \equiv \hat{\sigma}_{i j} \hat{\sigma}^{i j}=\sum_{i} \dot{\beta}_{i}^{2} .
$$

É importante enfatizar que, uma vez que estamos usando a métrica $\gamma_{i j}$ para manipular os índices espaciais, segue que

$$
\begin{aligned}
\left(\gamma^{i j}\right)^{\cdot} & =-\gamma^{i k} \gamma^{j l} \gamma_{k l}=-2 \hat{\sigma}^{i j} \\
(\dot{\gamma})^{i j} & =\gamma^{i k} \gamma^{j l}\left(\gamma_{k l}\right)^{\cdot}=+2 \hat{\sigma}^{i j}
\end{aligned}
$$

onde usamos $\left(\gamma^{i k} \gamma_{k j}\right)^{\cdot}=0$ na primeira dessas equações.

\subsection{Equações de Friedmann e Soluções Gerais}

\subsubsection{Equações de Friedmann}

Supondo que o conteúdo de matéria do universo seja completamente descrito pelo tensor de energia-momento

$$
T_{\mu \nu}=\rho u_{\mu} u_{\nu}+p\left(g_{\mu \nu}+u_{\mu} u_{\nu}\right)+\pi_{\mu \nu},
$$

onde $\rho$ é a densidade de energia do fluido em questão, $p$ sua pressão isotrópica e $\pi_{\mu \nu}$ o estresse anisotrópico (tal que $\pi_{\mu \nu} u^{\mu}=0$ e $\pi_{\mu}^{\mu}=0$ ), as equações de Einstein tomam a seguinte forma

$$
\begin{aligned}
3 H^{2} & =\kappa \rho+\frac{1}{2} \hat{\sigma}^{2}, \\
\frac{\ddot{S}}{\bar{S}} & =-\frac{\kappa}{6}(\rho+3 p)-\frac{1}{3} \hat{\sigma}^{2}, \\
\left(\hat{\sigma}_{j}^{i}\right)^{\cdot} & =-3 H \hat{\sigma}_{j}^{i}+\kappa \tilde{\pi}_{j}^{i} .
\end{aligned}
$$


Quando combinadas, essas equações levam à equação de conservação da matéria

$$
\dot{\rho}+3 H(\rho+p)+\hat{\sigma}_{i j} \tilde{\pi}^{i j}=0,
$$

na qual os componentes ij do tensor $\pi_{\mu \nu}$ foram definidos como $S^{2} \tilde{\pi}_{i j}$ (de modo que $\left.\tilde{\pi}_{j}^{i}=\gamma^{i k} \tilde{\pi}_{k j}\right)$.

Dado que estamos interessados na dinâmica de um período inflacionário do universo, assumiremos que o conteúdo material do mesmo é descrito por um único campo escalar homogêneo $(\varphi=\varphi(t))$, descrito por

$$
T_{\mu \nu}=\partial_{\mu} \varphi \partial_{\nu} \varphi-\left(\frac{1}{2} \partial_{\alpha} \varphi \partial^{\alpha} \varphi+V\right) g_{\mu \nu}, \quad \pi_{\mu \nu}=0 .
$$

Da equação de conservação $\nabla_{\mu} T^{\mu \nu}=0$ segue a equação de Klein-Gordon que, incidentemente, mantém a mesma forma encontrada em universos isotrópicos

$$
\ddot{\varphi}+3 H \dot{\varphi}+V, \varphi=0 .
$$

onde $V,_{\varphi} \equiv \mathrm{d} V / \mathrm{d} \varphi$. Dessa forma as equações de Einstein se reescrevem na forma

$$
\begin{aligned}
3 H^{2} & =\kappa\left[\frac{1}{2} \dot{\varphi}^{2}+V(\varphi)\right]+\frac{1}{2} \hat{\sigma}^{2}, \\
\frac{\ddot{S}}{S} & =-\frac{\kappa}{3}\left[\dot{\varphi}^{2}-V(\varphi)\right]-\frac{1}{3} \hat{\sigma}^{2}, \\
\left(\hat{\sigma}_{j}^{i}\right)^{\cdot} & =-3 H \hat{\sigma}_{j}^{i} .
\end{aligned}
$$

nas quais definimos $\kappa \equiv 8 \pi G$. A última destas equações pode ser facilmente integrada

$$
\hat{\sigma}_{j}^{i}=\frac{\mathcal{K}_{j}^{i}}{S^{3}}
$$

sendo que $\mathcal{K}_{j}^{i}$ é um tensor constante, $\left(\mathcal{K}_{j}^{i}\right)^{\cdot}=0$. O resultado acima implica que

$$
\hat{\sigma}^{2}=\frac{\mathcal{K}^{2}}{S^{6}}, \quad \mathcal{K}^{2}=\mathcal{K}_{j}^{i} \mathcal{K}_{i}^{j}
$$

de onde podemos deduzir que

$$
\dot{\hat{\sigma}}=-3 H \hat{\sigma} \text {. }
$$

Adotando a definição (2.3) para o parâmetro de $e$-folds da inflação, esta última equação nos mostra que $\hat{\sigma} \propto e^{-3 N}$. Daqui resulta que, para um modelo típico de inflação com $N \simeq 70$, o componente de cisalhamento do espaço no final da inflação é da ordem de $10^{92}$ vezes menor que seu valor inicial. Essa isotropização absoluta do espaço é, aliás, um dos sucessos do modelo inflacionário.

\subsubsection{Tempo Conforme}

Assim como no caso isotrópico, podemos também introduzir um parâmetro de "tempo conforme" $\mathrm{d} \eta \equiv S^{-1} \mathrm{~d} t$, a partir do qual a métrica (5.3) se reescreve como

$$
\mathrm{d} s^{2}=S^{2}(\eta)\left[-\mathrm{d} \eta^{2}+\gamma_{i j}(\eta) \mathrm{d} x^{i} \mathrm{~d} x^{j}\right] .
$$


A partir do fator de escala médio $S(\eta)$, definiremos o parâmetro de Hubble conforme como $\mathcal{H} \equiv S^{\prime} / S$, onde a linha refere-se à derivada com relação ao parâmetro $\eta$. O tensor de cisalhamento no tempo conforme

$$
\sigma_{i j} \equiv \frac{1}{2} \gamma_{i j}^{\prime}
$$

relaciona-se a $\hat{\sigma}_{i j}$ através da relação $\sigma_{i j}=S \hat{\sigma}_{i j}$, de modo que a quantidade $\sigma^{2} \equiv \sigma_{i j} \sigma^{i j}$ fica agora caracterizada por $\sigma^{2}=\sum_{i=1}^{3}\left(\beta_{i}^{\prime}\right)^{2}$ e relaciona-se a $\hat{\sigma}$ através de $\sigma=S \hat{\sigma}$. As "equações de Friedmann" no tempo conforme são

$$
\begin{aligned}
\mathcal{H}^{2} & =\frac{\kappa}{3}\left[\varphi^{\prime 2}+V(\varphi) S^{2}\right]+\frac{1}{6} \sigma^{2} \\
\mathcal{H}^{\prime} & =-\frac{\kappa}{3}\left[\varphi^{\prime 2}-V(\varphi) S^{2}\right]-\frac{1}{3} \sigma^{2} \\
\left(\sigma_{j}^{i}\right)^{\prime} & =-2 \mathcal{H} \sigma_{j}^{i},
\end{aligned}
$$

já a equação de conservação do campo fica

$$
\varphi^{\prime \prime}+2 \mathcal{H} \varphi^{\prime}+S^{2} V_{\varphi}=0
$$

\subsubsection{Soluções Gerais}

Podemos encontrar algumas soluções gerais para o sistema de equações acima em termos do fator de escala médio $S$. Para tanto, reescrevamos as quantidades $\beta_{i}$ na forma

$$
\beta_{i}=B_{i} W(t)
$$

onde $B_{i}$ são constantes a serem determinadas. Assim sendo, as equações (5.9) e (5.23) implicam em

$$
\left(\sum B_{i}^{2}\right) \dot{W}^{2}(t)=\frac{\mathcal{K}^{2}}{S^{6}}
$$

de onde segue que

$$
W(t)=\int \frac{\mathrm{d} t}{S^{3}}
$$

Os vínculos (5.5) e (5.9), por sua vez, forçam os coeficientes $B_{i}$ a obedecerem as relações

$$
\sum_{i=1}^{3} B_{i}=0, \quad \sum_{i=1}^{3} B_{i}^{2}=\mathcal{K}^{2},
$$

as quais podem ser facilmente satisfeitas através da parametrização

$$
B_{i}=\sqrt{\frac{2}{3}} \mathcal{K} \sin \alpha_{i}, \quad \text { sendo } \quad \alpha_{i}=\alpha+\frac{2 \pi}{3} i, \quad i \in\{1,2,3\}
$$

Portanto, as soluções gerais para as funções $\beta_{i}(t)$ em termos do fator de escala são:

$$
\beta_{i}(t)=\sqrt{\frac{2}{3}} \mathcal{K} \sin \left(\alpha+\frac{2 \pi}{3} i\right) \times \int \frac{\mathrm{d} t}{S^{3}},
$$


sendo que $S$ é solução de

$$
3 H^{2}=\kappa \rho+\frac{1}{2} \frac{\mathcal{K}^{2}}{S^{6}}
$$

Uma vez especificada uma equação de estado $p=p(\rho)$, a equação de conservação nos fornece $\rho$ com função de $S$. Isso nos permite, em princípio, extrair $S(t)$ da equação (5.37).

No decorrer do trabalho a quantidade $\hat{\sigma} / H$ surgirá de forma recorrente. Portanto será conveniente introduzir uma segunda quantidade de cisalhamento, ou "cisalhamento reduzido", através de

$$
x \equiv \frac{1}{\sqrt{6}} \frac{\hat{\sigma}}{H}=\frac{1}{\sqrt{6}} \frac{\sigma}{\mathcal{H}}
$$

em termos da qual a equação de Friedmann fica $\left(1-x^{2}\right) H^{2}=\kappa \rho / 3$. Vale notar que a condição de positividade local de energia requer $x^{2}<1$.

\subsubsection{Caso Particular: Constante Cosmológica}

Apresentaremos agora o caso ilustrativo de uma constante cosmológica pura, definida através de $V=$ constante e $\dot{\varphi}=0$. Como veremos, esse caso será de grande relevância durante o estágio que precede o período de rolagem lenta da inflação, dado que a dependência do tensor de cisalhamento com o fator de escala médio sugere que, inicialmente, $\hat{\sigma} \gg V(\varphi) \gg \dot{\varphi}$. Se esse não for o caso, então o campo se encontrará no seu regime de rolagem rápida, e sua densidade de energia se comportará como $S^{-3}$, exatamente como o escalar de cisalhamento.

Para o caso de uma constante cosmológica, a equação de Friedmann toma a seguinte forma

$$
H^{2}=V_{0}\left[1+\left(\frac{S_{*}}{S}\right)^{6}\right]
$$

$\operatorname{com}^{1} V_{0} \equiv \kappa V / 3$ e $S_{*} \equiv\left(\mathcal{K}^{2} / 6 V_{0}\right)^{1 / 6}$. Esta equação tem como solução

$$
S(t)=S_{*}\left[\sinh \left(t / \tau_{*}\right)\right]^{1 / 3}
$$

onde introduzimos um tempo característico

$$
\tau_{*}^{-1} \equiv 3 \sqrt{V_{0}} .
$$

Dessa forma, obtemos da equação (5.33) que

$$
W(t)=W_{0}+\frac{\tau_{*}}{S_{*}^{3}} \log \left[\tanh \left(\frac{t}{2 \tau_{*}}\right)\right],
$$

sendo que a constante $W_{0}$ pode ser, sem perda de generalidade, igualada a zero (escolha que corresponde a uma redefinição do instante inicial $t_{0}$ ). Daqui segue, usando $\sqrt{3 / 2} \mathcal{K}=S_{*}^{3} / \tau_{*}$, que os fatores de escalas direcionais se comportam como

$$
X_{i}(t)=S_{*}\left[\sinh \left(\frac{t}{\tau_{*}}\right)\right]^{1 / 3}\left[\tanh \left(\frac{t}{2 \tau_{*}}\right)\right]^{\frac{2}{3} \sin \alpha_{i}} .
$$

\footnotetext{
${ }^{1}$ Notemos as dimensões das quantidades em questão: $\rho \sim M^{4}, G \sim M^{-2}, H \sim M, \hat{\sigma} \sim M$, $B_{i} \sim M, W \sim M^{-1}, V_{0} \sim M^{2}, S_{*} \sim M^{0}$ onde $M$ é a escala de massa.
} 
A partir desta expressão, podemos deduzir também as leis de evolução dos parâmetros de Hubble direcionais

$$
h_{i}(t)=\frac{1}{3 \tau_{*}} \frac{1}{\sinh \left(t / \tau_{*}\right)}\left[2 \sin \alpha_{i}+\cosh \left(t / \tau_{*}\right)\right] .
$$

O parâmetro médio de Hubble e o cisalhamento reduzido ficam determinados respectivamente por

$$
H(t)=\frac{1}{3 \tau_{*}} \frac{1}{\tanh \left(t / \tau_{*}\right)}, \quad x(t)=\frac{1}{\cosh \left(t / \tau_{*}\right)} .
$$

O comportamento temporal dos fatores de escala direcionais são mostrados na Fig. 5.1 para vários valores do parâmetro livre $\alpha$ restritos ao intervalo $\alpha \in[0,2 \pi / 3]$. Como podemos ver nessa figura, para $\alpha<\pi / 2$ a direção $i=3$ sofre um ricochete. Para o caso particular $\alpha=\pi / 2$, nenhuma das direções sofre contração. Fica claro, portanto, que sempre existirá uma direção sujeita a um ricochete, exceto no caso particular em que $\alpha=\pi / 2$. Ao longo desse trabalho, as figuras apresentadas terão como referência o valor $\alpha=\pi / 4$.
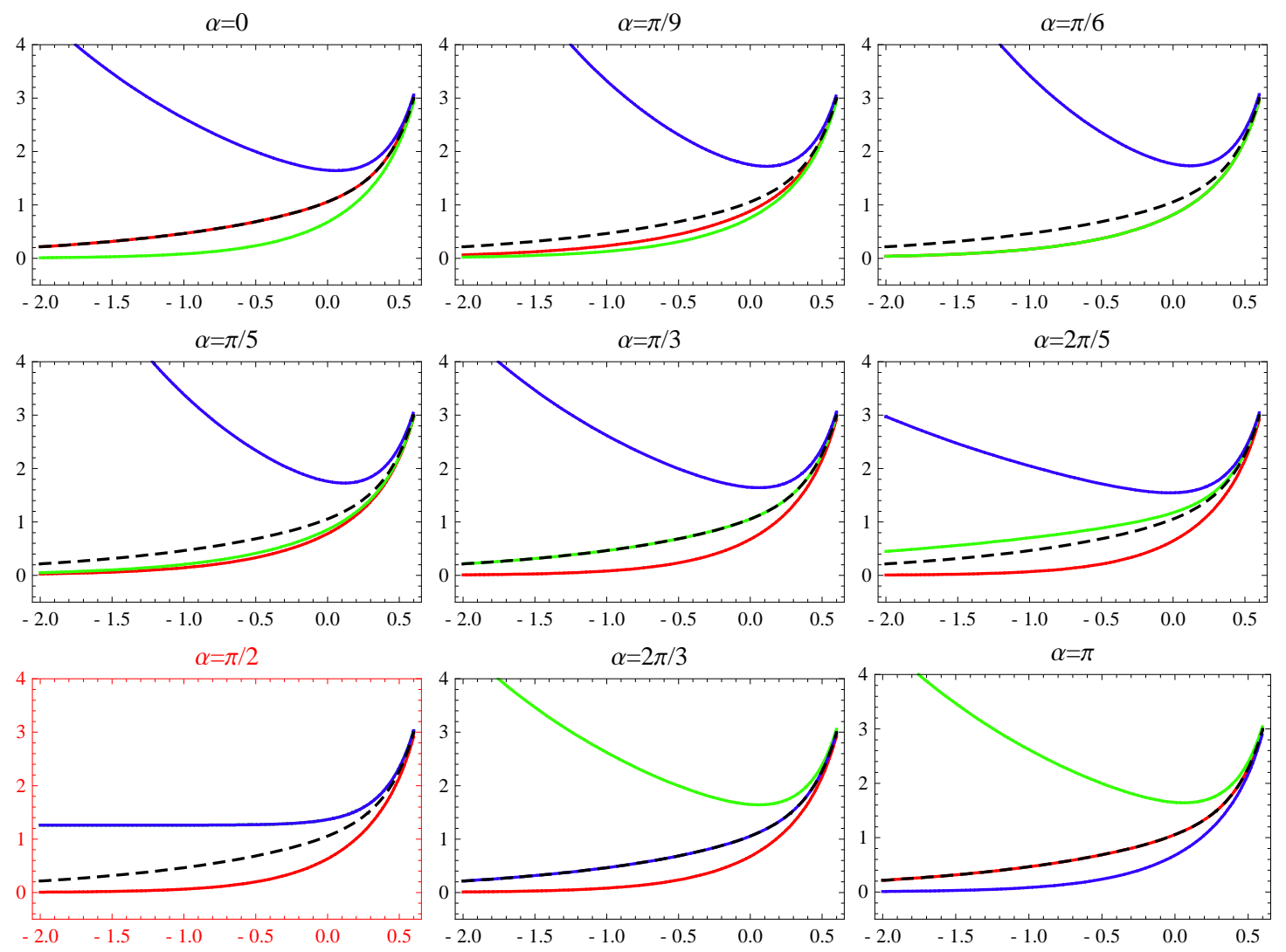

Figura 5.1: Evolução temporal dos fatores de escala direcionais de acordo com diferentes valores do parâmetro $\alpha$ em função de $\log (m t)$. A figura mostra os três fatores de escala direcionais e o fator de escala médio $S$ (linha tracejada), todos em unidades de $S_{*}$. Note que os três fatores de escala direcionais são permutados entre si quando $\alpha$ sofre uma variação de $2 \pi / 3$, e que existe dois casos particulares em que o espaçotempo tem simetria planar, dados por $\alpha=\pi / 6$ e $\alpha=\pi / 2$. O último caso é ainda mais peculiar, dado que ele representa o único universo do tipo Bianchi I em que nenhuma das direções sofre contração. 
Podemos calcular o tempo de duração do ricochete em função do ângulo $\alpha$. Considerando que $h_{i}\left(t_{\text {ric }}\right)=0$ no instante exato $t=t_{\text {ric }}$ do ricochete, temos

$$
t_{\text {ric }}=\tau_{*} \cosh ^{-1}\left(-2 \sin \alpha_{i}\right) \text {. }
$$

A Fig. 5.2 mostra os instantes em que acontece o ricochete em função dos valores de $\alpha$. Note que os mesmos são sempre menores que $1.4 \tau_{*}$.
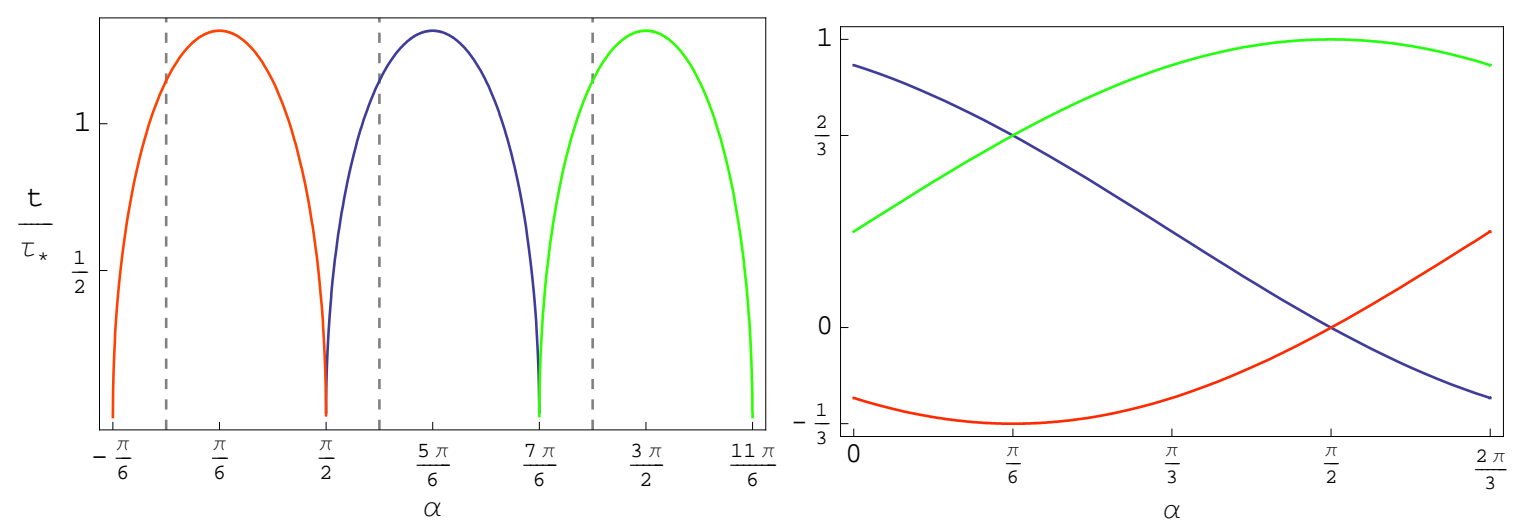

Figura 5.2: A figura da esquerda mostra os instantes em que as direções $i=1$ (azul), $i=2$ (vermelho) e $i=3$ (verde) ricocheteiam. Em $\alpha=\pi / 2$ nenhuma das direções se contrai e, para valores maiores de $\alpha$, ocorre uma permutação das direções que se contraem. À direita vemos os valores dos expoentes de Kasner em função do parâmetro $\alpha$ que caracteriza os universos Bianchi I próximos à singularidade.

\section{Comportamento próximo à singularidade}

Qualquer que seja o potencial escolhido para modelar o período de inflação, a equação de Friedmann será dominada pelo cisalhamento perto da singularidade $(S=0)$. Isso significa que podemos usar as soluções obtidas para o caso de uma constante cosmológica para ilustrar o comportamento do universo nesse regime. Da análise anterior, vemos que

$$
X_{i}(t)=S_{*}\left(\frac{t}{2 \tau_{*}}\right)^{\frac{2}{3} \sin \alpha_{i}+\frac{1}{3}}\left[1+\frac{1}{18}\left(1-\sin \alpha_{i}\right)\left(\frac{t}{\tau_{*}}\right)^{2}+\mathcal{O}\left(\left(\frac{t}{\tau_{*}}\right)^{4}\right)\right]
$$

Próximo à singularidade, a métrica (5.1) pode, portanto, ser expandida em torno de uma solução de Kasner, da forma

$$
\mathrm{d} s_{\text {Kasner }}^{2}=-\mathrm{d} t^{2}+S_{*}^{2} \sum_{i=1}^{3}\left(\frac{t}{2 \tau_{*}}\right)^{2 p_{i}}\left(\mathrm{~d} x^{i}\right)^{2}
$$

ondes os coeficientes

$$
p_{i}(\alpha)=\frac{2}{3} \sin \alpha_{i}+\frac{1}{3}
$$

satisfazem $\sum_{i} p_{i}=\sum_{i} p_{i}^{2}=1$ (veja a Fig. 5.2). Portanto, nesse regime, teremos

$$
\mathrm{d} s^{2} \simeq-\mathrm{d} t^{2}+S_{*}^{2} \sum_{i=1}^{3}\left(\frac{t}{2 \tau_{*}}\right)^{2 p_{i}}\left[1+\frac{1}{12}\left(1-p_{i}\right)\left(\frac{t}{\tau_{*}}\right)^{2}\right]\left(\mathrm{d} x^{i}\right)^{2}
$$


além de correções quárticas da forma $\left(t / \tau_{*}\right)^{4}$. É interessante calcular os invariantes geométricos da métrica próximos à singularidade

$$
\begin{aligned}
R & =\frac{4}{3 \tau_{*}^{2}} \\
R_{\mu \nu} R^{\mu \nu} & =\frac{4}{9 \tau_{*}^{4}} \\
R_{\mu \nu \rho \sigma} R^{\mu \nu \rho \sigma} & =\frac{1}{27 \tau_{*}^{4}}\left\{8+\frac{4}{\cosh ^{4}\left[t /\left(2 \tau_{*}\right)\right]}+\frac{32 \cosh \left(t / \tau_{*}\right)}{\sinh ^{4}\left(t / \tau_{*}\right)}[\sin (3 \alpha)+1]\right\}
\end{aligned}
$$

Aqui vemos claramente que as quantidades $R$ e $R_{\mu \nu} R^{\mu \nu}$ são regulares na singularidade, o que é de fato esperado para um universo dominado por uma constante cosmológica, uma vez que $R_{\mu \nu}=\Lambda g_{\mu \nu}$. Já o invariante $R_{\mu \nu \rho \sigma} R^{\mu \nu \rho \sigma}$ diverge quando nos aproximamos da singularidade em $t=0$, exceto no caso particular $\alpha=\pi / 2$, que corresponde ao ramo positivo considerado em [23]. Nesse caso particular, a métrica de Kasner tem a tripla $(1,0,0)$ de coeficientes. É importante enfatizar que o espaço-tempo caracterizado pelo parâmetro $\alpha=\pi / 2$ corresponde a um ponto singular no conjunto dos espaços-tempo de Bianchi I, dado que nesse caso não existe uma convergência uniforme dos invariantes da métrica calculados próximos à singularidade quando $\alpha \rightarrow \pi / 2$.

\subsection{Regime de Rolagem Lenta}

Passemos agora à caracterização do regime de rolagem lenta da inflação no caso anisotrópico. Para essa finalidade definiremos os parâmetros de rolagem lenta da mesma maneira como feito na seção $§ 2.2 .1$

$$
\epsilon \equiv 3 \frac{\varphi^{\prime 2}}{\varphi^{\prime 2}+2 S^{2} V}, \quad \delta \equiv 1-\frac{\varphi^{\prime \prime}}{\mathcal{H} \varphi^{\prime}}=-\frac{\ddot{\varphi}}{H \dot{\varphi}} .
$$

Em termos dessas definições, as equações de Friedmann e de Klein-Gordon no tempo conforme se reescrevem na forma

$$
\left(1-x^{2}\right) \mathcal{H}^{2}=\frac{\kappa}{3-\epsilon} V S^{2}, \quad(3-\delta) \mathcal{H} \varphi^{\prime}+V_{\varphi} S^{2}=0
$$

Um pouco de manipulação algébrica fornece

$$
\frac{\mathcal{H}^{\prime}}{\mathcal{H}^{2}}=(1-\epsilon)+(\epsilon-3) x^{2}, \quad \frac{S^{\prime \prime}}{S}=\mathcal{H}^{2}\left[2-\epsilon+(\epsilon-3) x^{2}\right]
$$

e, de modo análogo para $x$, encontramos

$$
x^{\prime}=-\mathcal{H} x\left(1-x^{2}\right)(3-\varepsilon) .
$$

A dinâmica dos parâmetros de rolagem lenta fica determinada pelas seguintes equações

$$
\begin{aligned}
\epsilon^{\prime} & =2 \mathcal{H} \epsilon(\epsilon-\delta) \\
\delta^{\prime} & =\mathcal{H}\left[-9 x^{2}+\frac{S^{2} V_{, \varphi \varphi}}{\mathcal{H}^{2}}\left(1-x^{2}\right)(3 \epsilon+3 \delta)+\delta\left(\delta+\epsilon\left(1-x^{2}\right)\right)\right] .
\end{aligned}
$$


Usando a definição (5.54) e a segunda equação em (5.55), o parâmetro $\epsilon$ pode ser reescrito na seguinte forma

$$
\epsilon=\frac{\left(1-x^{2}\right)}{2 \kappa}\left(\frac{V_{, \varphi}}{V}\right)^{2}\left(\frac{3-\epsilon}{3-\delta}\right)^{2}
$$

A partir desta expressão e da relação $\dot{\varphi}^{2}=2 \epsilon V /(3-\epsilon)$, deduzimos que

$$
\dot{\varphi}=-\frac{1}{\sqrt{\kappa}} \sqrt{\frac{(3-\epsilon)\left(1-x^{2}\right)}{(3-\delta)^{2}}} \frac{V_{, \varphi}}{\sqrt{V}} .
$$

Portanto, enquanto as condições $\dot{\varphi}^{2} \ll V(\varphi)$ e $x \simeq 1$ correspondentes ao regime de domínio do cisalhamento que antecede a inflação forem satisfeitas, teremos

$$
\epsilon \rightarrow 0, \quad \delta \rightarrow-3
$$

Notemos aqui que, diferentemente do caso isotrópico, o limite assintótico para o parâmetro $\delta$ é -3, e não zero. Isso significa que inicialmente, mesmo que o cisalhamento do espaço decresça rapidamente, o campo escalar $\varphi$ permanece aproximadamente constante (de acordo com a eq. (5.60)) e $\delta$ permanece próximo de -3 . Esta solução converge, em $t \rightarrow 0$, à solução de uma constante cosmológica encontrada em §5.2.4.

Por outro lado, pode acontecer que o sistema de equações se encontre inicialmente num regime transitório em que $\dot{\varphi}^{2} \gg V$. Se este for o caso, teremos os seguintes comportamentos assintóticos

$$
\epsilon \rightarrow 3, \quad \delta \rightarrow 3, \quad \dot{\varphi} \simeq \frac{\varphi_{0}}{t}
$$

Ou seja, a velocidade do campo diminui com o passar do tempo e esta solução novamente converge ao atrator do regime de rolagem lenta. Como veremos, tais resultados gerais sobre limites assintóticos serão importantes na compreensão da dinâmica do inflaton.

\subsection{Inflação Caótica: integração numérica}

Nesse trabalho nos concentraremos no exemplo específico da chamada inflação caótica, caracterizada por um potencial da forma

$$
V=\frac{1}{2} m^{2} \varphi^{2}
$$

Quando esse é caso, as equações dinâmicas (5.19) e (5.18) ficam

$$
\begin{aligned}
& h^{2}=\frac{1}{6}\left[\frac{1}{2} \dot{\psi}^{2}+\psi^{2}+\left(\frac{S_{*}}{S}\right)^{6}\right] \\
& \ddot{\psi}+3 h \dot{\psi}+\psi=0,
\end{aligned}
$$

onde introduzimos o parâmetro $\tau=m t$ como variável de tempo adimensional. Em termos do novo tempo, temos $h=H / m, \psi=\varphi / M_{\mathrm{p}}$ (onde $M_{\mathrm{p}}^{-2}=8 \pi G=\kappa$ ) e 
$S_{*}=(\mathcal{K} / m)^{1 / 3}$. Escrito desta forma, fica claro que a especificação completa das várias soluções do sistema (5.64-5.65) depende de três números: $\left\{\psi\left(t_{0}\right), \dot{\psi}\left(t_{0}\right), S_{*}\right\}$. Como veremos, essa dependência extra do parâmetro $S_{*}$ faz com que o sistema dinâmico de equações (5.64-5.65) se comporte de modo diferente do seu análogo em espaços-tempo do tipo FRW ${ }^{2}$.

É um resultado bem conhecido que a dinâmica do inflaton possui soluções com propriedades atratoras. Essas soluções são tais que, para uma grande classe de condições iniciais, ocorre uma convergência das soluções para o regime de rolagem lenta definido por $\dot{\varphi} \simeq$ constante. No diagrama do espaço de fase essas soluções aparecem como curvas horizontais que, ao final do regime atrator, oscilam em torno do ponto de mínimo do potencial $V(\varphi)$. A dinâmica inflacionária em universos do tipo Bianchi I possui o mesmo comportamento atrator, embora este seja quantitativamente diferente durante a fase em que o conteúdo de energia do universo é dominado pelo cisalhamento do espaço. Durante o período inicial, as soluções são rapidamente atraídas aos pontos onde $\dot{\varphi} \simeq 0$ e $\varphi \simeq$ constante. Esse regime atrator é caracterizado pelas soluções apresentadas em (5.67-5.71). À medida que o cisalhamento decai, o sistema converge para o regime atrator usual típico de um universo isotrópico, como mostra a Fig. 5.4.

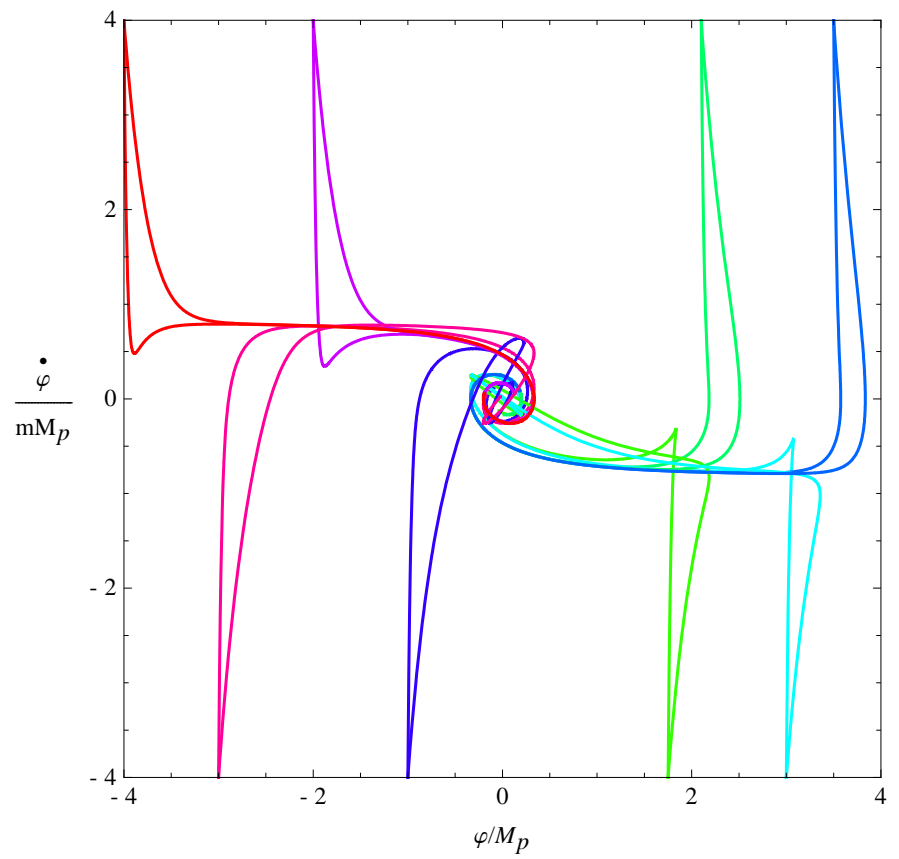

Figura 5.3: Espaço de fase das soluções inflacionárias para o caso Bianchi I. As curvas que se sobrepõem correspondem aos mesmos valores de $\dot{\varphi}$ e $\varphi$ e diferentes valores para $\hat{\sigma}_{0}$. (veja a Fig. 5.4 para uma representação tridimensional do mesmo diagrama). É instrutivo comparar essa figura à Fig. 2.4 do caso isotrópico.

Em suma, temos aqui um mecanismo de dupla atração, caracterizado pela transição

\footnotetext{
${ }^{2}$ Esse resultado também pode ser visto da seguinte maneira: o termo proporcional a $S^{-6}$ na equação (5.64) é matematicamente equivalente à contribuição de um campo escalar sem massa, $\chi$, cuja dinâmica é determinada pela equação de Klein-Gordon $\ddot{\chi}+3 h \dot{\chi}=0$ e que tem como solução $\dot{\chi} \propto S^{-3}$. A solução geral do sistema de equações também requer condições iniciais para este grau de liberdade extra.
} 
do universo de Bianchi I para o universo de FRW e, em seguida, desse para o regime de rolagem lenta da inflação.

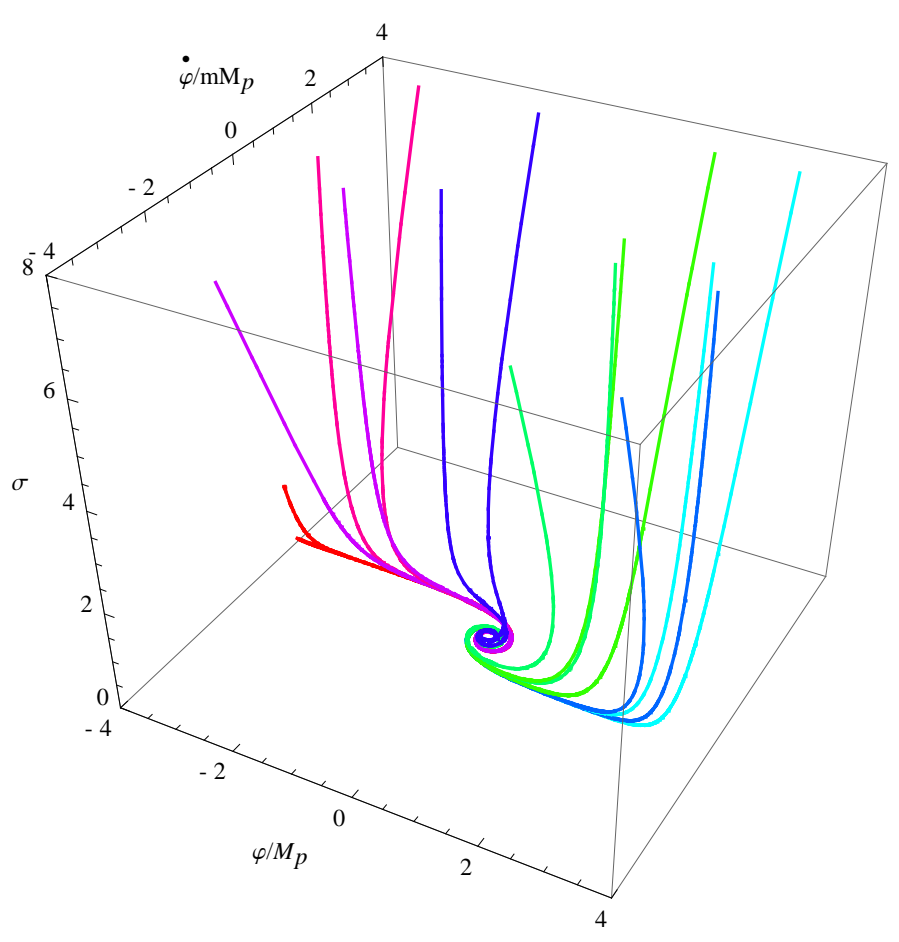

Figura 5.4: Espaço de fase tridimensional $\{\varphi, \dot{\varphi}, \sigma\}$ de universos inflacionários do tipo Bianchi I. O plano $\sigma=0$ corresponde ao limite de cisalhamento nulo (limite de FRW, Fig. 5.3). A figura ilustra o mecanismo de dupla atração do sistema.
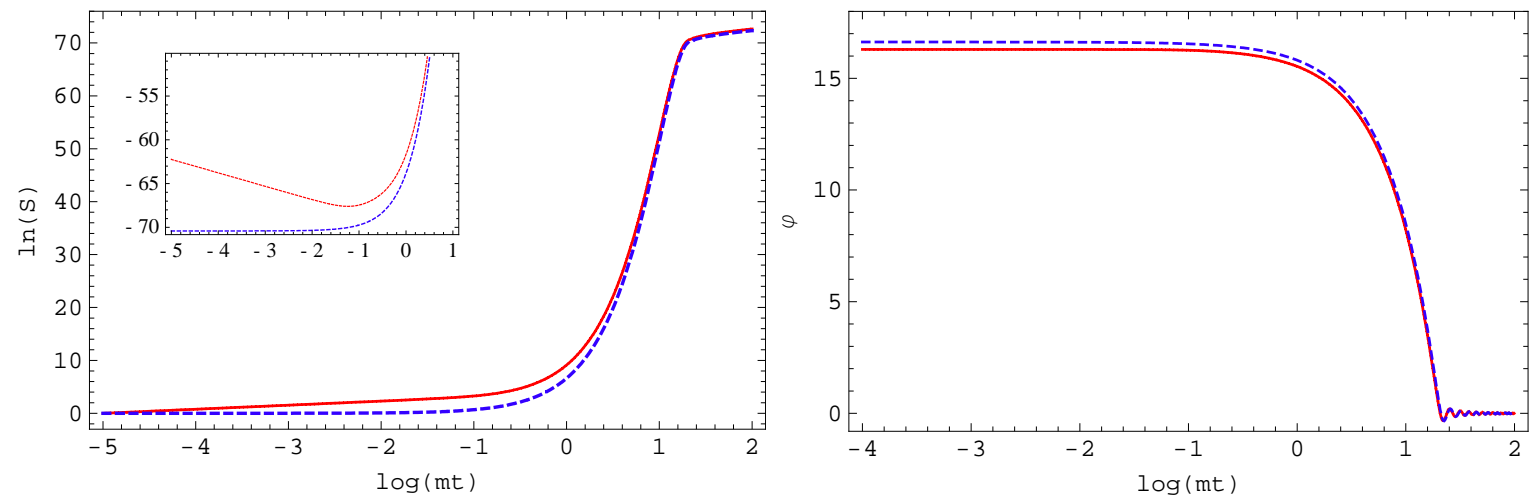

Figura 5.5: Evolução do fator de escala médio (esquerda) e do campo escalar (direita) em função do tempo para os universos de Bianchi I (linha sólida, vermelha) e FRW (linha tracejada, azul). As soluções para o caso Bianchi I correspondem a $\varphi_{0}=16 M_{\mathrm{p}}$. Para o caso FRW a solução foi normalizada de tal forma que os fatores de escala tenham o mesmo valor no final da inflação (período em que o cisalhamento do espaço é nulo). A figura interior no painel esquerdo mostra a evolução logarítmica da velocidade de expansão em ambos os casos. O mínimo da curva vermelha refere-se ao instante em que $\ddot{S}=0$ no caso Bianchi I. 
A Fig. 5.5 mostra a dinâmica da fase inflacionária para ambos os espaços-tempo de Bianchi I e FRW. Enquanto em ambos os casos o fator de escala médio é uma função crescente do tempo, o efeito de um período primordial de cisalhamento se traduz como um decréscimo da velocidade de expansão no caso Bianchi I. Por outro lado, a dinâmica do campo escalar é fracamente afetada pela presença do cisalhamento do espaço.

Consideremos agora em detalhes o comportamento do sistema de equações próximo à singularidade. Para tanto, definamos um novo instante característico $\tau_{*}$ através de

$$
\tau_{*}=\sqrt{\frac{2}{3}} \frac{M_{\mathrm{p}}}{m \varphi_{0}} .
$$

Desenvolvendo as equações deste sistema em séries de potência no tempo até a segunda ordem, obtemos

$$
\begin{aligned}
H(t) & =\frac{1}{3 t}\left[1+\frac{1}{3} \frac{t^{2}}{\tau_{*}^{2}}+\mathcal{O}\left(\frac{t^{4}}{\tau_{*}^{4}}\right)\right], \\
x(t) & =1-\frac{1}{2} \frac{t^{2}}{\tau_{*}^{2}}+\mathcal{O}\left(\frac{t^{4}}{\tau_{*}^{4}}\right), \\
\varphi(t) & =\varphi_{0}\left[1-\frac{1}{6}\left(\frac{M_{\mathrm{p}}}{\varphi_{0}}\right)^{2} \frac{t^{2}}{\tau_{*}^{2}}+\mathcal{O}\left(\frac{t^{4}}{\tau_{*}^{4}}\right)\right], \\
\delta(t) & =-3\left[1-\frac{1}{2} \frac{t^{2}}{\tau_{*}^{2}}+\mathcal{O}\left(\frac{t^{4}}{\tau_{*}^{4}}\right)\right], \\
\epsilon(t) & =\frac{1}{3}\left(\frac{M_{\mathrm{p}}}{\varphi_{0}}\right)^{2} \frac{t^{2}}{\tau_{*}^{2}}+\mathcal{O}\left(\frac{t^{4}}{\tau_{*}^{4}}\right),
\end{aligned}
$$

em perfeito acordo com o resultado encontrado em [23]. Escrito dessa forma, fica fácil entender o comportamento assintótico $\delta \rightarrow-3$ próximo à singularidade. Isso decorre simplesmente do fato de que o campo escalar evolui com o quadrado do tempo. Vale lembrar que esta situação é substancialmente diferente do caso encontrado em universos isotrópicos onde o campo varia linearmente com o tempo. Neste casos, temse

$$
\varphi=\varphi_{i}\left[1-\left(\frac{M_{\mathrm{p}}}{\varphi_{i}}\right)^{2} \frac{t}{\tau_{*}}\right], \quad S(t)=S_{i} \exp \left\{\frac{1}{M_{\mathrm{p}}^{2}}\left[\varphi_{i}^{2}-\varphi(t)^{2}\right]\right\}
$$

e os parâmetros de rolagem lenta ficam determinados por

$$
\epsilon=2 \frac{M_{\mathrm{p}}^{2}}{\varphi^{2}}, \quad \delta=0
$$

Voltemos agora aos parâmetros de rolagem lenta introduzidos na seção $§ 5.3$. No caso particular de um potencial quadrático (definido em (5.63)), temos

$$
\delta^{\prime}=\mathcal{H}(3-\delta)\left[\frac{\epsilon^{2}-3 \delta}{3-\epsilon}-x^{2}(3-\epsilon)\right]
$$

dado que $S^{2} V_{, \varphi \varphi}=\epsilon \mathcal{H}^{2}(3-\delta)^{2} /(3-\epsilon)$. Durante o período de domínio do cisalhamento do espaço, $\delta \sim-3$ e, de acordo com a equação anterior, vemos que $\delta$ permanece constante e que $\epsilon^{\prime}$, segundo a equação (5.57), tem a mesma ordem de grandeza que 
$\epsilon$. Esse resultado difere do encontrado em modelos isotrópicos, onde em geral tem-se $\left(\epsilon^{\prime}, \delta^{\prime}\right) \sim\left(\epsilon^{2}, \delta^{2}\right)$, respectivamente (veja a equação (2.37)). Aqui, vemos que a variação de $\epsilon$ não pode ser negligenciada antes que $\delta$ tenha convergido para zero. Uma vez tendo o universo se isotropizado e enquanto houver inflação, ambos $\epsilon$ e $\delta$ convergem para seus valores usuais. A Fig. 5.6 ilustra a evolução desses dois parâmetros e os compara ao caso isotrópico de FRW.
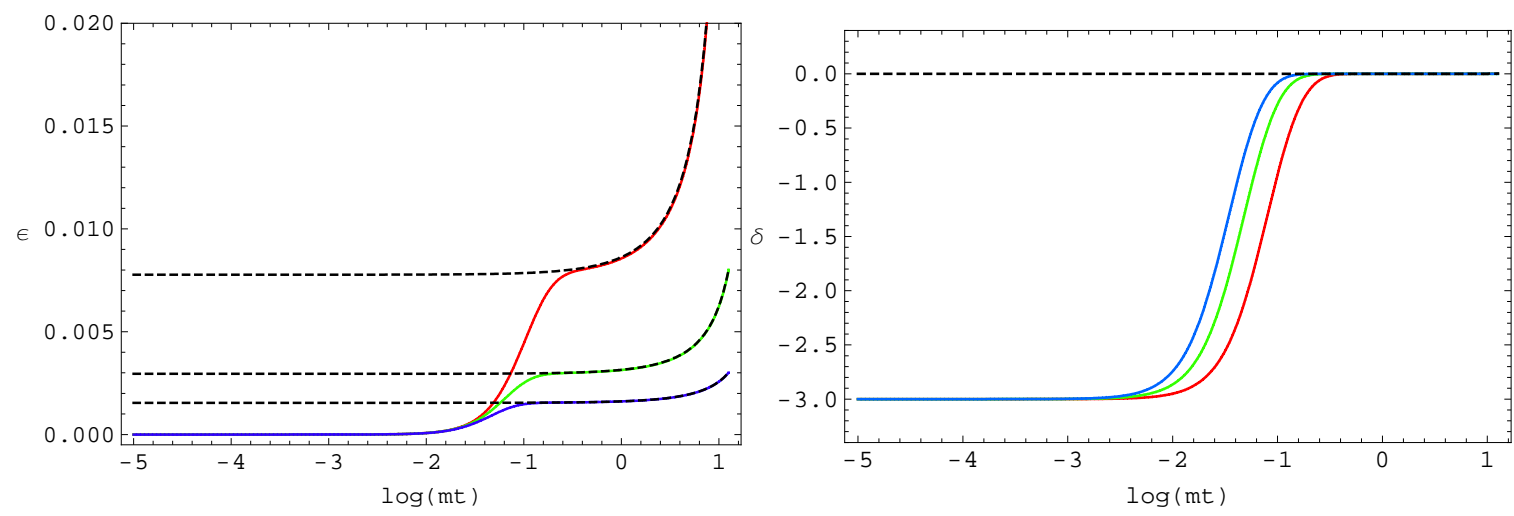

Figura 5.6: Evolução dos parâmetros de rolagem lenta $\epsilon$ (esquerda) e $\delta$ (direita) durante o período inflacionário para ambos os casos Bianchi I (linhas contínuas) e FRW (linhas tracejadas). As figuras correspondem ao potencial da forma (5.63) e os valores iniciais do campo são $\varphi_{0}=(16,26,36) M_{\mathrm{p}}$ correspondendo respectivamente às curvas vermelha, verde e azul. Para o modelo anisotrópico utilizamos $\alpha=\pi / 4$.

Para um dado valor do campo escalar, $\varphi_{0}$, definiremos $\varphi_{i}$ como sendo o valor do campo no instante em que o universo começa a inflar, ou seja, no instante em que a condição $\ddot{S}=0$ é satisfeita. Dessa forma, o número de $e$-folds do período inflacionário fica determinado por

$$
N\left[\varphi_{i}\right] \equiv \ln \frac{S\left(\varphi_{f}\right)}{S\left(\varphi_{i}\right)}=\int_{\varphi_{i}}^{\varphi_{f}} \frac{H}{\dot{\varphi}} \mathrm{d} \varphi,
$$

onde $\varphi_{f}$ é definido como o valor do campo no instante em que $\epsilon \simeq 1$. Levando em conta que o cisalhamento é completamente negligenciável no fim da inflação, $\varphi_{f}$ fica determinado por

$$
\varphi_{f}=\sqrt{2} M_{\mathrm{p}} .
$$

Em vista desse período pré-inflacionário, é útil definir uma quantidade que caracterize a duração do mesmo em termos dos valores iniciais do campo. Definamos então

$$
\Delta \varphi\left[\varphi_{0}\right] \equiv \varphi_{0}-\varphi_{i}\left[\varphi_{0}\right],
$$

como sendo a quantidade que indica quanto o campo se moveu ao longo do potencial antes do início do período inflacionário. Assim sendo, podemos quantificar a duração do período inflacionário unicamente através de $\varphi_{0}$

$$
N\left[\varphi_{0}\right]=\int_{\varphi_{i}\left(\varphi_{0}\right)}^{\sqrt{2} M_{\mathrm{p}}} \frac{H}{\dot{\varphi}} \mathrm{d} \varphi .
$$

Essa expressão deve ser comparada à sua análoga no caso de FRW

$$
N_{\mathrm{FL}}\left[\varphi_{i}\right]=\frac{1}{4}\left(\frac{\varphi_{i}}{M_{\mathrm{p}}}\right)^{2}-\frac{1}{2} .
$$


A evolução da função (5.77) está representada na Fig. 5.7, onde vemos nitidamente que, quanto maior o valor inicial do campo escalar, menor é o impacto do período primordial de cisalhamento do universo. À direita desta figura encontra-se um gráfico do número de e-folds para os casos de FRW e Bianchi I. Como esperado, o impacto do período primordial de cisalhamento sobre o número de $e$-folds é pequeno.
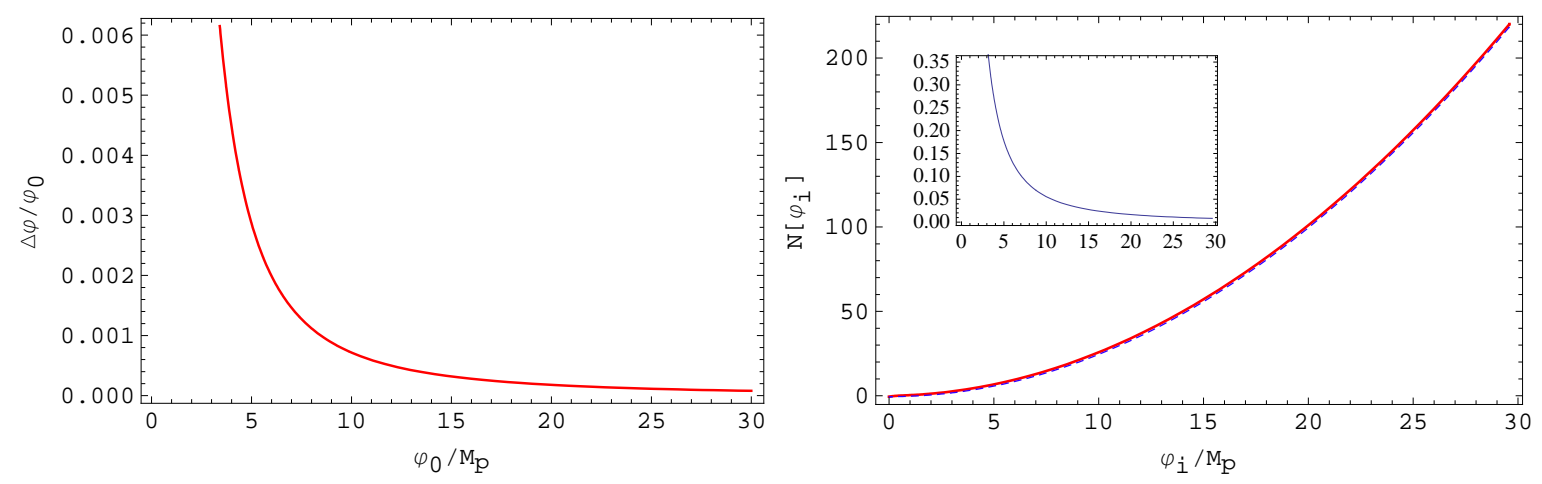

Figura 5.7: Variação relativa do campo escalar durante a fase de domínio do cisalhamento em função do seu valor inicial (esquerda). Comparação do número de $e$-folds para universos de Bianchi I (linha sólida, vermelha) e FRW (linha azul, tracejada) durante o período inflacionário como função de $\varphi_{i}$ (direita). A figura interior mostra a diferença relativa entre $N$ e $N_{\text {FRW }}$ em função de $\varphi_{i}$.

\subsection{Discussão}

O estudo das propriedades homogêneas de um universo anisotrópico que acabamos de apresentar mostra que, de maneira geral, sempre existirá um direção espacial sujeita a um ricochete, exceto no caso particular $\alpha=\pi / 2$ considerado em [23]. Essa característica decorre do fato de que o efeito de cisalhamento conserva elementos de volume comóveis. Dado que existem direções que se expandem, esta conservação de volume requer forçosamente que ao menos uma das outras direções se contraia. No que diz respeito à dinâmica do universo, as soluções aproximadas (5.67-5.71) reproduzem fielmente as soluções exatas encontradas numericamente.

Esta análise também mostra que o efeito de cisalhamento é efetivo tipicamente até o instante característico $\tau_{*}$ introduzido em (5.66). À medida que voltamos no tempo, o universo converge rapidamente para uma singularidade inicial e é, portanto, geodesicamente incompleto (da mesma forma como em outros modelos inflacionários [90]). De fato, conforme discutiremos mais adiante, não queremos aqui extrapolar nosso modelo para além do tempo de Planck ou de alguma outra escala temporal fundamental. Portanto assumiremos simplesmente que nosso modelo é uma boa aproximação para o universo inflacionário depois desta singularidade inicial.

Com relação aos parâmetros de rolagem lenta, nossa análise mostrou que a variação temporal de $\epsilon$ não pode ser negligenciada antes que o universo tenha se isotropizado por completo. Mostramos também que o campo escalar pouco se move durante o período de cisalhamento e que, para o mesmo valor inicial do campo, o número de $e$-folds permanece praticamente inalterado na presença do cisalhamento do espaço. 


\section{CAPÍTULO 6}

\section{Dinâmica Perturbativa}

No capítulo anterior apresentamos uma descrição semi-analítica detalhada do regime inflacionário em universos anisotrópicos a partir de uma perspectiva exata, ou seja, sem aproximações. Neste capítulo iremos construir a teoria inflacionária das perturbações cosmológicas em universos anisotrópicos. Nosso objetivo final é encontrar as equações dinâmicas de evolução dos modos escalares, vetoriais e tensoriais neste contexto.

\subsection{Decomposição dos modos perturbativos}

Essa seção será dedicada à construção de um conjunto completo de variáveis invariantes de calibre capazes de descrever as perturbações cosmológicas em universos anisotrópicos. Seguiremos aqui um procedimento à la Bardeen, análogo ao que foi feito na primeira parte desta tese. Tendo em vista a importância de uma definição precisa dos modos escalares, vetoriais e tensoriais, será necessário trabalhar no espaço de Fourier. Portanto, iniciaremos esta seção definindo nossas integrais de Fourier e salientando suas diferenças com as definições usualmente feitas em universos isotrópicos.

\subsubsection{Definição das transformações de Fourier}

Dada uma função do tempo conforme $\eta$ e de um sistema de coordenadas comóveis $\left\{x^{j}\right\}$ definido sobre hipersuperfícies de tempo constante, $f\left(x^{j}, \eta\right)$, definiremos sua decomposição em modos de Fourier através de

$$
f\left(x^{j}, \eta\right)=\int \frac{\mathrm{d}^{3} k}{(2 \pi)^{3 / 2}} f\left(k_{i}, \eta\right) e^{\mathrm{i} k_{i} x^{i}}
$$

cuja inversa é

$$
f\left(k_{j}, \eta\right)=\int \frac{\mathrm{d}^{3} x}{(2 \pi)^{3 / 2}} f\left(x^{i}, \eta\right) e^{-\mathrm{i} k_{i} x^{i}} .
$$

No espaço de Fourier, os covetores comóveis de onda, $k_{i}$, são constantes no tempo. Decorre daí que no espaço-tempo de Bianchi I os contra-vetores comóveis são funções 
do tempo, $k^{i} \equiv \gamma^{i j} k_{j}$. Diferentemente do que ocorre no caso de FRW, é importante não confundir as quantidades $k_{i}$ e $k^{i}$, uma vez que essa distinção não comuta com a evolução temporal. No entanto a combinação $k_{i} x^{i}=k^{i} x_{i}$ é sempre constante, de modo que não existe uma dependência temporal extra em nossas definições (6.1) e (6.2). Podemos verificar, usando a definição (5.10), que

$$
\left(k^{i}\right)^{\prime}=-2 \sigma^{i j} k_{j} .
$$

Isso implica que o módulo quadrado do vetor de onda comóvel, $k^{2}=k^{i} k_{i}=\gamma^{i j} k_{i} k_{j}$, é agora uma função do tempo. Sua taxa de variação é dada explicitamente por

$$
\frac{k^{\prime}}{k}=-\sigma^{i j} \hat{k}_{i} \hat{k}_{j}
$$

onde introduzimos o vetor unitário $\hat{k}_{i} \equiv k_{i} / k$. Este vetor será de grande utilidade para nossa análise e, portanto, escreveremos também sua derivada

$$
\left(\hat{k}^{i}\right)^{\prime}=\left(\sigma^{j l} \hat{k}_{j} \hat{k}_{l}\right) \hat{k}^{i}-2 \sigma^{i j} \hat{k}_{j} .
$$

Pode ser facilmente verificado, em todas as expressões acima, que no limite de um espaço-tempo isotrópico, $k^{i}$ e $k_{i}$ são constantes.

\subsubsection{Decomposição EVT}

O espírito da decomposição nos diferentes modos perturbativos é essencialmente o mesmo do caso isotrópico apresentado em $§ 3.2$. No entanto a dependência temporal da métrica se estende aos vetores de base do espaço de Fourier e, portanto, aos operadores de projeção dos modos EVT.

Um campo escalar $f$ qualquer é, por definição, um modo escalar puro e portanto sua decomposição EVT é trivial. Dado um campo vetorial tridimensional $V^{i}$, podemos decompô-lo na forma

$$
V_{i}=\partial_{i} V+\bar{V}_{i}, \quad \text { onde } \quad \partial^{i} \bar{V}_{i}=0 .
$$

Notemos que a derivada parcial (no lugar de uma derivada covariante) é consequência da nossa escolha de um sistema de coordenadas ortogonais, porém não cartesiano, para as seções espaciais da métrica. No espaço de Fourier, essa decomposição fica

$$
V_{i}=k_{i} V+\bar{V}_{i}, \quad \text { onde } \quad k^{i} \bar{V}_{i}=0 .
$$

A condição de transversalidade do modo vetorial $\bar{V}_{i}$ implica que seus componentes vivem num subespaço de dimensão 2 perpendicular ao vetor $k_{i}$. Consideremos portanto a base $\left\{\boldsymbol{e}^{1}, \boldsymbol{e}^{2}\right\}$ do subespaço perpendicular a esse vetor. Por construção, essa base satisfaz as seguintes condições de ortogonalidade

$$
e_{i}^{a} e_{j}^{b} \gamma^{i j}=\delta^{a b}, \quad e_{i}^{a} k^{i}=0
$$

Essas condições definem a base a menos de uma arbitrariedade na rotação dos vetores $\left\{\boldsymbol{e}^{1}, \boldsymbol{e}^{2}\right\}$ em torno do vetor $k_{i}$. Em termos desses vetores, o modo vetorial em (6.7) pode ser decomposto como

$$
\bar{V}_{i}\left(k_{j}, \eta\right)=\sum_{a=1}^{2} V_{a}\left(k_{j}, \eta\right) e_{i}^{a}\left(k_{j}\right)
$$


o que define os dois graus de liberdade vetoriais $V_{a}$. A dependência dos modos vetoriais em $k_{i}$ decorre da dependência da base $\left\{\boldsymbol{e}^{1}, \boldsymbol{e}^{2}\right\}$ com $k_{i}$, uma vez que para diferentes vetores de ondas temos diferentes vetores de base. Os dois vetores de base acima definidos nos permitem construir um operador de projeção sobre o espaço perpendicular a $\hat{k}_{i}$ na forma

$$
P_{i j} \equiv e_{i}^{1} e_{j}^{1}+e_{i}^{2} e_{j}^{2}=\gamma_{i j}-\hat{k}_{i} \hat{k}_{j} .
$$

Esse operador satisfaz as condições $P^{i k} P_{k j}=P_{j}^{i}, P_{j}^{i} k^{j}=0$ e $P^{i j} P_{i j}=2 . \quad P_{i j}$ é também um projetor dos modos vetoriais, de modo que a decomposição (6.7) pode ser totalmente reescrita em termos do vetor original

$$
V_{i}=\left(\hat{k}_{j} V^{j}\right) \hat{k}_{i}+P_{i}^{j} V_{j}
$$

De maneira análoga, qualquer campo tensorial simétrico (em 3 dimensões) $V_{i j}$ pode ser decomposto em suas partes escalares, vetoriais e tensoriais

$$
V_{i j}=T \gamma_{i j}+D_{i j} S+2 \partial_{(i} \bar{V}_{j)}+2 \bar{V}_{i j}
$$

sendo que

$$
\partial_{i} \bar{V}^{i}=0, \quad \bar{V}_{i}^{i}=0=\partial_{i} \bar{V}^{i j} \quad \text { e } \quad D_{i j} \equiv \partial_{i} \partial_{j}-\frac{1}{3} \nabla^{2} \gamma_{i j} .
$$

Nesta decomposição, o tensor simétrico $\bar{V}_{i j}$ é transverso $\left(k^{i} \bar{V}_{i j}=0\right)$ e de traço nulo $\left(\bar{V}_{i}^{i}=0\right)$. Portanto, este tensor pode ser reescrito da seguinte maneira

$$
\bar{V}_{i j}\left(k_{l}, \eta\right)=\sum_{\lambda=+, \times} V_{\lambda}\left(k_{l}, \eta\right) \varepsilon_{i j}^{\lambda}\left(k_{l}\right)
$$

onde os tensores de polarização $\varepsilon_{i j}^{\lambda}$ estão definidos de acordo com

$$
\varepsilon_{i j}^{\lambda}=\frac{e_{i}^{1} e_{j}^{1}-e_{i}^{2} e_{j}^{2}}{\sqrt{2}} \delta_{+}^{\lambda}+\frac{e_{i}^{1} e_{j}^{2}+e_{i}^{2} e_{j}^{1}}{\sqrt{2}} \delta_{\times}^{\lambda} .
$$

Verifica-se facilmente usando (6.8) que os tensores de polarização são transversos $\left(k^{i} \varepsilon_{i j}^{\lambda}=0\right)$, têm traço nulo $\left(\gamma^{i j} \varepsilon_{i j}^{\lambda}=0\right)$ e são ortogonais entre si $\left(\varepsilon_{i j}^{\lambda} \varepsilon_{\mu}^{i j}=\delta_{\mu}^{\lambda}\right)$.

Para manipular corretamente os tensores de polarização, será bastante conveniente introduzir dois novos operadores

$$
Q_{i j} \equiv e_{i}^{1} e_{j}^{2}-e_{i}^{2} e_{j}^{1}, \quad \text { e } \quad \eta_{\lambda \mu} \equiv \delta_{\lambda}^{+} \delta_{\mu}^{\times}-\delta_{\mu}^{+} \delta_{\lambda}^{\times} .
$$

Segue desta definição que o tensor $Q_{i j}$ satisfaz

$$
P_{i j} Q^{i j}=0 \quad \text { e } \quad Q_{i j} Q^{i j}=2 .
$$

Tais propriedades nos permitem reescrever o produto entre dois e três tensores de polarização na forma

$$
\varepsilon_{i k}^{\lambda} \varepsilon_{j}^{k \mu}=\frac{1}{2}\left(P_{i j} \delta^{\lambda \mu}+Q_{i j} \eta^{\lambda \mu}\right), \quad \varepsilon_{i k}^{\lambda} \varepsilon_{\mu}^{k j} \varepsilon_{j \nu}^{i}=0 .
$$

Introduziremos agora um operador de projeção dos modos tensoriais

$$
\Lambda_{i j}^{k l} \equiv P_{i}^{k} P_{j}^{l}-\frac{1}{2} P_{i j} P^{k l},
$$


e um segundo operador com a propriedade de "extração do traço"

$$
T_{j}^{i} \equiv \hat{k}^{i} \hat{k}_{j}-\frac{1}{3} \delta_{j}^{i}
$$

Assim, os modos escalares, vetoriais e tensoriais contidos na decomposição (6.12) podem ser reescritos na seguinte forma

$$
V_{i j}=\frac{1}{3}\left(\gamma^{k l} V_{k l}\right) \gamma_{i j}+\frac{3}{2}\left(V_{k l} T^{k l}\right) T_{i j}+2 \hat{k}_{(i}\left(P_{j)}^{k} \hat{k}^{l} V_{k l}\right)+\Lambda_{i j}^{k l} V_{k l} .
$$

Portanto, mostramos que dada uma equação vetorial na forma $V_{i}=0$, podemos sempre extrair seu modo escalar através de $\hat{k}_{i} V^{i}=0$ e seus modos vetoriais através de $P_{j}^{i} V_{i}=0$. Além disso, dada qualquer equação tensorial na forma $V_{i j}=0$, podemos extrair seus modos escalares através de $\gamma^{i j} V_{i j}=0$ e $T^{i j} V_{i j}=0$, e seus modos vetoriais e tensoriais mediante a aplicação dos projetores $P_{l}^{i} \hat{k}^{j}$ e $\Lambda_{i j}^{k l}$, respectivamente.

\subsubsection{Propriedades dos projetores e dos modos vetoriais e ten- soriais}

A decomposição em modos escalares, vetoriais e tensoriais que acabamos de apresentar é a mesma usada na primeira parte dessa tese. Porém, quando o espaço-tempo é anisotrópico, a extração desses modos deve ser feita com cuidado pois, uma vez que a métrica espacial $\gamma_{i j}$ depende do tempo, o processo de extração dos modos nem sempre comuta com a evolução temporal dos mesmos. Em particular, a dependência temporal de $\gamma_{i j}$ e as condições de transversalidade $\hat{k}^{i} e_{i}^{a}=0=\hat{k}^{i} \bar{\varepsilon}_{i j}$ implicam que os vetores e tensores de polarização devem também ser funções do tempo. De fato, a condição $\left(k_{i} e_{a}^{i}\right)^{\prime}=0$ implica que $k_{i}\left(e_{a}^{i}\right)^{\prime}=0$, ou seja, o vetor $\left(e_{i}^{a}\right)^{\prime}$ pode ser escrito como uma combinação linear de $\boldsymbol{e}^{1}$ e $\boldsymbol{e}^{2}$

$$
\left(e_{a}^{i}\right)^{\prime}=\sum_{a} \mathcal{R}_{a b} e_{b}^{i}
$$

Em cada hipersuperfície de tempo constante existe uma liberdade na escolha da base, conseqüente da liberdade de rotação da mesma em torno de $\hat{k}_{i}$. Essa liberdade pode ser eliminada impondo-se que a matriz $\mathcal{R}_{a b}$ seja simétrica, $\mathcal{R}_{[a b]}=0$. Dessa forma, a condição de ortogonalidade $\left(e_{a}^{i} e_{i}^{b}\right)^{\prime}=0$ implica que

$$
\mathcal{R}_{a b}=-\sigma_{i j} e_{a}^{i} e_{b}^{j} .
$$

Conseqüentemente, a derivada temporal dos vetores de polarização fica determinada por

$$
\left(e_{i}^{a}\right)^{\prime}=\sum_{b} \mathcal{R}_{a b} e_{i}^{b}+2 \sigma_{i j} e_{a}^{j}
$$

de onde podemos deduzir imediatamente que

$$
k^{i}\left(e_{i}^{a}\right)^{\prime}=2 \sigma_{i j} k^{i} e_{a}^{j}
$$

Isso também nos permite deduzir a evolução temporal dos tensores de polarização $\varepsilon_{i j}^{\lambda}$. Partindo da definição (6.15), obtemos

$$
\left(\varepsilon_{i j}^{\lambda}\right)^{\prime}=-\left(\sigma^{k l} \varepsilon_{k l}^{\lambda}\right) P_{i j}-\left(\sigma^{k l} P_{k l}\right) \varepsilon_{i j}^{\lambda}+4 \sigma_{(i}^{k} \varepsilon_{j) k}^{\lambda}
$$


Dessa expressão segue imediatamente que

$$
k^{i} k^{j}\left(\varepsilon_{i j}^{\lambda}\right)^{\prime}=0, \quad \gamma^{i j}\left(\varepsilon_{i j}^{\lambda}\right)^{\prime}=2 \sigma^{i j} \varepsilon_{i j}^{\lambda}, \quad k^{i}\left(\varepsilon_{i j}^{\lambda}\right)^{\prime}=2 \sigma^{i l} k_{l} \varepsilon_{i j}^{\lambda} .
$$

Apresentamos no Apêndice B mais algumas relações úteis dos vetores e tensores de polarização. Para a dedução do sistema final das equações de movimento, precisaremos também de duas novas matrizes. São elas

$$
\begin{aligned}
\mathcal{M}_{a b}^{\lambda} & \equiv \varepsilon_{i j}^{\lambda} e_{a}^{i} e_{b}^{j}=\mathcal{M}_{b a}^{\lambda} \\
\mathcal{N}_{a b} & \equiv Q_{i j} e_{a}^{i} e_{b}^{j}=-\mathcal{N}_{b a}
\end{aligned}
$$

Vale enfatizar que os índices $(a, b, c \ldots)$ e $(\lambda, \mu, \nu \ldots)$ são índices de quantidades definidas num subespaço do espaço de Fourier e, portanto, são transparentes às manipulações da métrica $\gamma_{i j}$.

\subsection{Variáveis invariantes de calibre}

Definidas as estruturas gerais dos projetores e do espaço onde serão construídas as equações de movimento dos modos perturbativos, passemos à construção das quantidades invariantes de calibre em espaços anisotrópicos. Esse procedimento nos levará a uma generalização das variáveis de Bardeen introduzidas em §3.3.1.

\subsubsection{Setor geométrico}

Consideremos a forma geral de uma métrica perturbada em torno da métrica de fundo (5.25). Assim como no caso isotrópico, o elemento de linha do espaço-tempo perturbado pode ser parametrizado na forma

$$
\mathrm{d} s^{2}=S^{2}\left[-(1+2 A) \mathrm{d} \eta^{2}+2 B_{i} \mathrm{~d} x^{i} \mathrm{~d} \eta+\left(\gamma_{i j}+h_{i j}\right) \mathrm{d} x^{i} \mathrm{~d} x^{j}\right],
$$

onde os campos $B_{i}$ e $h_{i j}$ são determinados por

$$
\begin{aligned}
B_{i} & =\partial_{i} B+\bar{B}_{i} \\
h_{i j} & =2 C \gamma_{i j}+2 \partial_{i} \partial_{j} E+2 \partial_{(i} E_{j)}+2 E_{i j}
\end{aligned}
$$

e satisfazem as condições de transversalidade (3.8) e (3.10). Uma transformação ativa do sistema de coordenadas definida por um dado campo vetorial $\xi^{\mu}$, como em (3.25), implica na seguinte lei de transformação da métrica

$$
g_{\mu \nu} \rightarrow g_{\mu \nu}+\mathcal{L}_{\xi} g_{\mu \nu}
$$

em que $\mathcal{L}_{\xi} g_{\mu \nu}$ é a derivada de Lie ao longo do vetor $\xi^{\mu}$. Em primeira ordem nas perturbações da métrica, decompondo-a na forma $g_{\mu \nu} \rightarrow g_{\mu \nu}+\delta g_{\mu \nu}$, encontramos

$$
\delta g_{\mu \nu} \rightarrow \delta g_{\mu \nu}+\mathcal{L}_{\xi} g_{\mu \nu} .
$$

Em seguida, decompomos o campo vetorial $\xi^{\mu}$ em seus modos EVT

$$
\xi^{0} \equiv T\left(x^{i}, \eta\right), \quad \xi^{i} \equiv \partial^{i} L\left(x^{i}, \eta\right)+L^{i}\left(x^{i}, \eta\right)
$$


onde $\partial_{i} L^{i}=0$. Utilizando a métrica (5.25) como nossa métrica de fundo, deduzimos que

$$
\begin{aligned}
\mathcal{L}_{\xi} g_{00} & =-2 S^{2}\left(T^{\prime}+\mathcal{H} T\right) \\
\mathcal{L}_{\xi} g_{0 i} & =S^{2}\left(\xi_{i}^{\prime}-\partial_{i} T-2 \sigma_{i j} \xi^{j}\right) \\
\mathcal{L}_{\xi} g_{i j} & =2 S^{2}\left(\partial_{(i} \xi_{j)}+\mathcal{H} T \gamma_{i j}+\sigma_{i j} T\right)
\end{aligned}
$$

Fazendo uso da expressão (6.33) e dos operadores de projeção definidos na seção anterior, encontramos as seguintes transformações de calibre para os diferentes modos perturbativos:

- Modos escalares

$$
\begin{aligned}
& A \rightarrow \widehat{A}=A+\frac{(S T)^{\prime}}{S}, \\
& B \rightarrow \widehat{B}=B-T+\frac{\left(k^{2} L\right)^{\prime}}{k^{2}}, \\
& C \rightarrow \widehat{C}=C+\mathcal{H} T-\frac{1}{2} \hat{k}^{i} \hat{k}^{j} \sigma_{i j} T, \\
& E \rightarrow \widehat{E}=E+L-\frac{3}{2} \frac{\hat{k}^{i} \hat{k}^{j}}{k^{2}} \sigma_{i j} T .
\end{aligned}
$$

- Modos vetoriais

$$
\begin{aligned}
& \bar{B}_{i} \rightarrow \widehat{\bar{B}_{i}}=\bar{B}_{i}+\gamma_{i j}\left(L^{j}\right)^{\prime}-2 \mathrm{i} \hat{k}^{j} \sigma_{j l} P_{i}^{l} L \\
& E_{i} \rightarrow \widehat{E_{i}}=E_{i}+L_{i}-i \frac{\hat{k}^{l}}{k} P_{i}^{j} \sigma_{j l} T .
\end{aligned}
$$

- Modos tensoriais

$$
E_{i j} \rightarrow \widehat{E_{i j}}=E_{i j}+T \Lambda_{i j}^{k l} \sigma_{k l}
$$

Estas transformações são bastante diferentes daquelas que encontramos em §3.3.1. Em particular, o modo tensorial não é mais um invariante de calibre. Notemos também que a lei de transformação para os modos vetoriais é diferente daquela encontrada na referência [53], onde a não-comutatividade entre os operadores de projeção e a evolução temporal dos modos foi negligenciada.

Temos agora duas alternativas para construir as variáveis de Bardeen. A primeira delas consiste na tarefa trabalhosa de procurar combinações das leis de transformação (6.38-6.44) que nos levem às quantidades invariantes. Uma outra possibilidade consiste em reparametrizar a métrica (6.29) da maneira seguinte [91]

$$
\begin{aligned}
B_{i} & =\partial_{i} B+\bar{B}_{i}+2 \sigma_{i j} \partial^{j} E \\
h_{i j} & =2 C\left(\gamma_{i j}+\frac{\sigma_{i j}}{\mathcal{H}}\right)+2 \partial_{i} \partial_{j} E+2 \partial_{(i} E_{j)}+2 E_{i j} .
\end{aligned}
$$

Em termos desta parametrização, as leis de transformação das funções da métrica tomam uma forma muito mais simples: 
- Modos escalares

$$
\begin{aligned}
& A \rightarrow \widehat{A}=A+\frac{(S T)^{\prime}}{S}, \\
& B \rightarrow \widehat{B}=B-T+L^{\prime} \\
& C \rightarrow \widehat{C}=C+\mathcal{H} T \\
& E \rightarrow \widehat{E}=E+L .
\end{aligned}
$$

- Modos vetoriais

$$
\begin{aligned}
& \bar{B}_{i} \rightarrow \widehat{\bar{B}_{i}}=\bar{B}_{i}+\gamma_{i j}\left(L^{j}\right)^{\prime} \\
& E_{i} \rightarrow \widehat{E_{i}}=E_{i}+L_{i} .
\end{aligned}
$$

- Modos tensoriais

$$
E_{i j} \rightarrow \widehat{E_{i j}}=E_{i j}
$$

Podemos notar que estas transformações são formalmente idênticas às transformações do caso isotrópico que encontramos em §3.3.1. Portanto, as variáveis de Bardeen também são as mesmas do caso isotrópico

$$
\begin{aligned}
\Phi & \equiv A+\frac{1}{S}\left[S\left(B-E^{\prime}\right)\right]^{\prime} \\
\Psi & \equiv-C-\mathcal{H}\left(B-E^{\prime}\right) \\
\Phi_{i} & \equiv \bar{B}_{i}-\gamma_{i j}\left(E^{j}\right)^{\prime} .
\end{aligned}
$$

Além disso, com essa reparametrização o modo tensorial é, por construção, invariante. Ambas as parametrizações sugeridas levam às mesmas equações dinâmicas. Porém, na prática, a segunda delas é mais simples, pois nesse caso as variáveis de Bardeen não dependem de fatores extras de $k$.

\subsubsection{Setor de matéria}

Focaremos nossa análise no caso em que o conteúdo material do universo é descrito por um campo escalar, uma vez que esse é o cenário de maior importância para as aplicações em inflação. Quando submetido à transformação de calibre (3.25), as perturbações do campo escalar $\varphi$ se transformam como $\delta \varphi \rightarrow \delta \varphi+\mathcal{L}_{\xi} \varphi$. Em primeira ordem nas perturbações, decompondo o campo na forma

$$
\varphi \rightarrow \varphi+\delta \varphi
$$

temos

$$
\delta \varphi \rightarrow \delta \varphi+\varphi^{\prime} T
$$

onde usamos (6.34). De modo análogo ao que foi feito com as variáveis geométricas, podemos definir uma função invariante de calibre para o setor de matéria através de

$$
\chi \equiv \delta \varphi+\left(B-E^{\prime}\right) \varphi^{\prime} .
$$

Usaremos também uma segunda definição de variável invariante de calibre para o setor de matéria

$$
Q \equiv \delta \varphi-C \frac{\varphi^{\prime}}{\mathcal{H}}
$$

relacionada à variável $\chi$ através de $Q=\chi+\Psi \varphi^{\prime} / \mathcal{H}$. 


\subsubsection{Calibre newtoniano}

Em analogia ao caso encontrado em FRW, definiremos o chamado calibre newtoniano através das condições

$$
B=\bar{B}^{i}=E=0 .
$$

Nesse calibre, portanto,

$$
A=\Phi, \quad C=-\Psi, \quad \chi=\delta \varphi, \quad \Phi^{i}=-\left(E^{i}\right)^{\prime},
$$

sendo que a última condição é equivalente a $\Phi_{i}=-E_{i}^{\prime}+2 \sigma_{i j} E^{j}$.

\subsection{Dinâmica das perturbações}

Definidas as variáveis invariantes de calibre, podemos passar à construção das equações de movimento dos modos perturbativos. A dedução da dinâmica de cada modo ficará mais transparente se decompusermos também o tensor de cisalhamento em termos dos seus componentes escalares, vetoriais e tensoriais, devidamente adaptados à base formada pelos vetores $\left\{\hat{\boldsymbol{k}}, \boldsymbol{e}^{1}, \boldsymbol{e}^{2}\right\}$. Começaremos portanto definindo a decomposição do tensor de cisalhamento e, em seguida, deduziremos as equações perturbadas de Einstein e Klein-Gordon.

\subsubsection{Decomposição do tensor de cisalhamento}

O tensor de cisalhamento $\sigma_{i j}$ é um tensor simétrico, tridimensional, de traço nulo. Conseqüentemente este tensor tem 5 componentes independentes. No sistema de coordenadas implícito em (5.3), $\sigma_{i j}$ tem apenas dois componentes independentes. Os três componentes restantes equivalem aos três ângulos de Euler necessários para reescrever nosso sistema de coordenadas numa forma mais geral.

Baseados em (6.12), decomporemos o tensor de cisalhamento da seguinte maneira

$$
\sigma_{i j}=\frac{3}{2}\left(\hat{k}_{i} \hat{k}_{j}-\frac{1}{3} \gamma_{i j}\right) \sigma_{\|}+2 \sum_{a=1,2} \sigma_{\mathrm{v} a} \hat{k}_{(i} e_{j)}^{a}+\sum_{\lambda=+, \times} \sigma_{\mathrm{T} \lambda} \varepsilon_{i j}^{\lambda} .
$$

Tal decomposição depende de cinco componentes independentes do cisalhamento numa base convenientemente adaptada à base $\left\{\hat{\boldsymbol{k}}, \boldsymbol{e}^{1}, \boldsymbol{e}^{2}\right\}$. No entanto, os componentes $\left(\sigma_{\|}, \sigma_{\mathrm{v} a}, \sigma_{\mathrm{T} \lambda}\right)$ não devem ser interpretados como os componentes de Fourier de $\sigma_{i j}$ - ainda que os mesmos dependam do versor $\hat{k}_{i}$ - pois essa dependência decorre da anisotropia local do espaço.

Algumas identidades importantes podem ser imediatamente deduzidas a partir da decomposição (6.63), como por exemplo

$$
\gamma^{i j} \sigma_{i j}=0, \quad \hat{k}^{j} \sigma_{i j}=\sigma_{\|} \hat{k}_{i}+\sum_{a} \sigma_{\mathrm{v} a} e_{i}^{a}, \quad \hat{k}^{i} \hat{k}^{j} \sigma_{i j}=\sigma_{\|}
$$

e também

$$
\hat{k}^{i} e_{a}^{j} \sigma_{i j}=\sigma_{\mathrm{v} a}, \quad \varepsilon_{\lambda}^{i j} \sigma_{i j}=\sigma_{\mathrm{T} \lambda}
$$


Em termos desses componentes, a contração completa do tensor de cisalhamento fica

$$
\sigma^{2}=\sigma^{i j} \sigma_{i j}=\frac{3}{2} \sigma_{\|}^{2}+2 \sum_{a} \sigma_{\mathrm{v} a}^{2}+\sum_{\lambda} \sigma_{\mathrm{T} \lambda}^{2}
$$

expressão esta que é independente de $\hat{k}_{i}$ e, portanto, uma função apenas do tempo.

\subsubsection{Dinâmica dos componentes do tensor de cisalhamento}

Na seção anterior, introduzimos a definição dos componentes do tensor de cisalhamento, $\sigma_{i j}$, numa base convenientemente definida no espaço de Fourier. A evolução temporal de cada um desses componentes pode ser extraída da equação (5.29) utilizando os operadores de projeção já definidos. Com um pouco de álgebra, obtemos

$$
\begin{aligned}
\sigma_{\|}^{\prime}+2 \mathcal{H} \sigma_{\|} & =-2 \sum_{a} \sigma_{\mathrm{v} a}^{2}, \\
\sigma_{\mathrm{v} a}^{\prime}+2 \mathcal{H} \sigma_{\mathrm{v} a} & =\frac{3}{2} \sigma_{\mathrm{v} a} \sigma_{\|}-\sum_{b, \lambda} \sigma_{\mathrm{v} b} \sigma_{\mathrm{T} \lambda} \mathcal{M}_{a b}^{\lambda}, \\
\sigma_{\mathrm{T} \lambda}^{\prime}+2 \mathcal{H} \sigma_{\mathrm{T} \lambda} & =2 \sum_{a, b} \sigma_{\mathrm{v} a} \sigma_{\mathrm{v} b} \mathcal{M}_{a b}^{\lambda},
\end{aligned}
$$

onde a matriz $\mathcal{M}_{a b}^{\lambda}$ foi definida em (6.27).

\subsubsection{Positividade da energia}

Antes de prosseguirmos adiante com a dedução das equações de movimento, apresentaremos uma análise simples envolvendo alguns vínculos na magnitude dos componentes do tensor $\sigma_{i j}$. Notemos inicialmente que a condição de positividade local da densidade de energia do inflaton, $\rho_{\varphi}>0$, implica, através de (5.27), que

$$
\frac{1}{6} \sigma^{2}=\mathcal{H}^{2}-\frac{\kappa}{3} \rho_{\varphi}<\mathcal{H}^{2}
$$

Por outro lado, segue da expressão (6.66) que $\sigma_{\|} / 2<\sigma / \sqrt{6}$, logo

$$
\sigma_{\|}<2 \mathcal{H}
$$

Essa propriedade irá garantir estabilidade e consistência ao nosso sistema de equações. Analogamente, é fácil mostrar que

$$
\sigma_{\mathrm{T} \lambda}<\sqrt{6} \mathcal{H} .
$$

Continuando com essa análise, notemos que as equações (6.67) e (6.69) implicam que

$$
\left|\frac{1}{S^{2}}\left(S^{2} \sigma_{\|}\right)^{\prime}\right|=2 \sum_{a} \sigma_{\mathrm{v} a}^{2} \leq \sigma^{2} \quad \text { e } \quad\left|\frac{1}{S^{2}}\left(S^{2} \sigma_{\mathrm{T} \lambda}\right)^{\prime}\right|=2 \sum_{a, b} \mathcal{M}_{a b}^{\lambda} \sigma_{\mathrm{v} a} \sigma_{\mathrm{v} b}<\frac{\sigma^{2}}{\sqrt{2}}
$$

de onde podemos concluir que

$$
\left|\frac{1}{S^{2}}\left(S^{2} \sigma_{\|}\right)^{\prime}\right|<6 \mathcal{H}^{2} \quad \text { e }\left|\frac{1}{S^{2}}\left(S^{2} \sigma_{\mathrm{T} \lambda}\right)^{\prime}\right|<3 \sqrt{2} \mathcal{H}^{2} .
$$

As relações (6.71-6.74) serão importantes para analisar a consistência das nossas equações no limite de pequenos comprimentos de onda. 


\subsubsection{Equação de Klein-Gordon}

A dinâmica do inflaton é descrita pela equação de Klein-Gordon, $g^{\mu \nu} \nabla_{\mu} \partial_{\nu} \varphi=V,_{\varphi}(\varphi)$, que pode também ser escrita na forma

$$
\frac{1}{\sqrt{-g}} \partial_{\mu}\left(\sqrt{-g} g^{\mu \nu} \partial_{\nu} \varphi\right)=V_{\varphi}(\varphi)
$$

Quando expandido em primeira ordem nas perturbações do campo, o lado direito da equação de Klein-Gordon fornece $V,_{\varphi}(\varphi+\chi)=V,_{\varphi}(\varphi)+V,_{\varphi \varphi}(\varphi) \chi$, onde $V,_{\varphi \varphi}$ representa a segunda derivada do potencial com relação ao campo. O lado esquerdo pode ser calculado diretamente se inserirmos as decomposições do campo e da métrica em (6.75) e proceder com a expansão até primeira ordem nas perturbações. Entretanto, olhando cuidadosamente para essa equação, vemos que o termo $\sqrt{-g}$ não pode contribuir para o cisalhamento do espaço, pois esse último tem traço nulo. Assim, em primeira ordem nas perturbações, a contribuição do cisalhamento para a equação de Klein-Gordon só pode surgir de $\delta g^{i j}$. Contudo, nesse caso, as perturbações da métrica estariam multiplicando $\partial_{i} \varphi(t)$, que é zero. Podemos então concluir que a equação para as perturbações do inflaton em Bianchi I será exatamente a mesma que encontramos em FRW, ou seja

$$
\chi^{\prime \prime}+2 \mathcal{H} \chi^{\prime}-\gamma^{i j} \partial_{i} \partial_{j} \chi+S^{2} V,_{\varphi \varphi} \chi=2\left(\varphi^{\prime \prime}+2 \mathcal{H} \varphi^{\prime}\right) \Phi+\varphi^{\prime}\left(\Phi^{\prime}+3 \Psi^{\prime}\right) .
$$

Esse resultado não se restringe ao caso particular de um campo escalar, uma vez que a equação de conservação de um fluido perfeito no espaço-tempo de Bianchi I não envolve o componente de cisalhamento e, portanto, é a mesma equação que encontramos em universos isotrópicos.

\subsubsection{Equações de Einstein}

O procedimento de decomposição das equações de Einstein consiste em partir da equação $\delta G_{\nu}^{\mu}=\kappa \delta T_{\nu}^{\mu}$ utilizando para isso as expressões (B.37-B.39) e (B.26-B.28) do Apêndice B. Em seguida decompomos essas equações em seus modos escalares, tensoriais e vetoriais utilizando os operadores de projeção já definidos. Esse procedimento requer um cuidado especial. No caso em que o universo é homogêneo e isotrópico, as operações de projeção dos modos comutam com a evolução temporal dos mesmos. Esse não é mais o caso em universos anisotrópicos. Consideremos o exemplo da extração de um modo vetorial contido numa equação da forma $\left(\Phi^{i}\right)^{\prime}+\mathcal{H} \Phi^{i}$. Inicialmente projetamos o modo vetorial desta equação através de

$$
P_{j}^{i}\left[\left(\Phi^{j}\right)^{\prime}+\mathcal{H} \Phi^{j}\right]
$$

Em seguida, decompomos o campo $\Phi^{j}$ através de (6.9) e multiplicamos essa equação por $e_{i}^{a}$

$$
e_{i}^{a} \sum_{b}\left[\left(\Phi^{b} e_{b}^{i}\right)^{\prime}+\mathcal{H} \Phi^{b} e_{b}^{i}\right]=\left(\Phi^{a}\right)^{\prime}-\left(e_{i}^{a}\right)^{\prime} \sum_{b}\left(\Phi^{b} e_{b}^{i}\right)+\mathcal{H} \Phi^{a} .
$$

Podemos então usar a equação (6.9) para reexpressar o termo $\left(e_{i}^{a}\right)^{\prime}$ e, em seguida, utilizar a decomposição (6.63) do tensor de cisalhamento. A dependência temporal 
da métrica $\gamma_{i j}$ implica, em particular, que os modos escalares, vetoriais e tensoriais estarão intimamente acoplados através de $\sigma_{i j}$.

De modo geral, a extração dos modos perturbativos das equações de Einstein é uma tarefa direta e trabalhosa. Esse procedimento resume-se em: (1) escrever as equações de Einstein no espaço de Fourier, (2) projetar os modos EVT através dos seus respectivos operadores de projeção, (3) comutar a projeção dos operadores com a evolução temporal dos vetores de base $\left\{\hat{\boldsymbol{k}}, \boldsymbol{e}^{1}, \boldsymbol{e}^{2}\right\}$ utilizando para esse fim a dinâmica dos vetores (6.23) e tensores (6.25) de polarização e, finalmente, (4) utilizar a decomposição (6.63) do tensor de cisalhamento. A seguir, apresentaremos os resultados desse processo.

\section{Modos escalares}

Existem quatro equações escalares que podem ser projetadas das equações de Einstein. A primeira delas resulta de $\delta G_{0}^{0}=\kappa \delta T_{0}^{0}$. Temos

$$
\begin{aligned}
& k^{2} \Psi+3 \mathcal{H}\left(\Psi^{\prime}+\mathcal{H} \Phi\right)-\frac{\kappa}{2}\left(\varphi^{\prime 2} \Phi-\varphi^{\prime} \chi^{\prime}-V,_{\varphi} S^{2} \chi\right)= \\
& \frac{1}{2} \sigma^{2}(X-3 \Psi)+\frac{1}{2} \frac{k^{2}}{\mathcal{H}} \sigma_{\|} \Psi-\frac{1}{2} k^{2} \sum_{a} \tilde{\sigma}_{\mathrm{v} a} \Phi_{a}-\frac{1}{2} \sum_{\lambda}\left[\sigma_{\mathrm{T} \lambda} E_{\lambda}^{\prime}+\left(\sigma_{\mathrm{T} \lambda}^{\prime}+2 \mathcal{H} \sigma_{\mathrm{T} \lambda}\right) E_{\lambda}\right]
\end{aligned}
$$

onde introduzimos duas novas variáveis

$$
X \equiv \Phi+\Psi+\left(\frac{\Psi}{\mathcal{H}}\right)^{\prime} \quad \text { e } \quad \sigma_{\mathrm{v} a} \equiv \mathrm{i} k \tilde{\sigma}_{\mathrm{v} a} .
$$

A segunda das equações escalares decorre da projeção $k^{i} \delta G_{i}^{0}=\kappa k^{i} \delta T_{i}^{0}$. Encontramos

$$
\Psi^{\prime}+\mathcal{H} \Phi-\frac{\kappa}{2} \varphi^{\prime} \chi=-\frac{1}{2 \mathcal{H}} \sigma^{2} \Psi+\frac{1}{2} \sigma_{\|} X+\frac{1}{2} \sum_{\lambda} \sigma_{\mathrm{T} \lambda} E_{\lambda}
$$

As duas equações restantes são obtidas das seguintes projeções

$$
\delta_{j}^{i} \delta G_{i}^{j}=\kappa \delta_{j}^{i} \delta T_{i}^{j} \quad \text { e } \quad\left(\hat{k}^{i} \hat{k}_{j}-\frac{1}{3} \delta_{j}^{i}\right) \delta T_{i}^{j}
$$

são elas:

$$
\begin{aligned}
& \Psi^{\prime \prime}+2 \mathcal{H} \Psi^{\prime}+\mathcal{H} \Phi^{\prime}+\left(2 \mathcal{H}^{\prime}+\mathcal{H}^{2}\right) \Phi-\frac{1}{3} k^{2}(\Phi-\Psi)+\frac{\kappa}{2}\left(\varphi^{\prime 2} \Phi-\varphi^{\prime} \chi^{\prime}+V,_{\varphi} S^{2} \chi\right)= \\
& -\frac{1}{2} \sigma^{2}(X-3 \Psi)+\frac{1}{6} \frac{k^{2}}{\mathcal{H}} \sigma_{\|} \Psi+\frac{1}{2} k^{2} \sum_{a} \tilde{\sigma}_{\mathrm{v} a} \Phi_{a}+\frac{1}{2} \sum_{\lambda}\left[\sigma_{\mathrm{T} \lambda} E_{\lambda}^{\prime}+\left(\sigma_{\mathrm{T} \lambda}^{\prime}+2 \mathcal{H} \sigma_{\mathrm{T} \lambda}\right) E_{\lambda}\right]
\end{aligned}
$$

e

$$
\frac{2}{3} k^{2}(\Phi-\Psi)=\sigma_{\|}\left(X-\frac{k^{2}}{3} \frac{\Psi}{\mathcal{H}}\right)+4 k^{2} \sum_{a, b, \lambda} \mathcal{M}_{a b}^{\lambda} \tilde{\sigma}_{\mathrm{v} a} \tilde{\sigma}_{\mathrm{v} b} E_{\lambda}-2 k^{2} \sum_{a} \tilde{\sigma}_{\mathrm{v} a} \Phi_{a}
$$

Pode-se verificar que, de fato, as equações (6.78-6.83) reduzem-se às equações (3.583.60) quando $\left(\sigma_{\|}, \sigma_{\mathrm{v} a}, \sigma_{\mathrm{T} \lambda}\right)=0$. 


\section{Modos vetoriais}

As equações de Einstein contribuem com duas equações para os modos vetoriais. Essas equações são obtidas através das seguintes projeções

$$
P_{j}^{i} \delta G_{i}^{0}=0, \quad \text { e } \quad P_{l}^{i} \hat{k}_{j} \delta G_{i}^{j}=0
$$

ou, de modo mais simples, através de $e_{a}^{i} \delta G_{i}^{0}=0$ e $e_{a}^{i} \hat{k}_{j} \delta G_{i}^{j}=0$. Essas projeções nos dão

$$
\Phi_{a}=-2 \tilde{\sigma}_{\mathrm{v} a} X+4 \sum_{b, \lambda} \mathcal{M}_{a b}^{\lambda} \tilde{\sigma}_{\mathrm{v} b} E_{\lambda}
$$

$\mathrm{e}$

$$
\begin{aligned}
\Phi_{a}^{\prime}+2 \mathcal{H} \Phi_{a} & -\frac{5}{2} \sigma_{\|} \Phi_{a}+\sum_{b, \lambda} \mathcal{M}_{a b}^{\lambda} \sigma_{\mathrm{T} \lambda} \Phi_{a}= \\
& -2 \tilde{\sigma}_{\mathrm{v} a} X^{\prime}+4 \sum_{b, \lambda} \mathcal{M}_{a b}^{\lambda} \tilde{\sigma}_{\mathrm{v} b} E_{\lambda}^{\prime}+4 \sum_{b, \lambda} \mathcal{N}_{a b} \tilde{\sigma}_{\mathrm{v} b}\left(\sigma_{\mathrm{T}+} \delta_{\lambda}^{\times}-\sigma_{\mathrm{T}} \delta_{\lambda}^{+}\right) E_{\lambda},
\end{aligned}
$$

sendo $\mathcal{N}_{a b}$ a matriz definida em (6.28). Devemos notar aqui um detalhe importante. Utilizando a equação (6.68), segue que a expressão (6.85) é solução da equação (6.86). Notemos também que a expressão (6.85) nada mais é que um vínculo que relaciona os modos escalar e tensoriais aos modos vetoriais. Isso mostra que os modos vetoriais não contribuem para a dinâmica das perturbações cosmológicas e, através desse vínculo, poderemos eliminá-los de maneira consistente do sistema final de equações.

\section{Modos tensoriais}

A equação para os modos tensoriais é obtida através de $\Lambda_{j l}^{i k} \delta G_{i}^{j}=0$ ou, de modo equivalente, através de $\varepsilon_{j}^{i \lambda} \delta G_{i}^{j}=0$. Visando simplificar os cálculos, usaremos a notação $(1-\lambda)$ para a polarização oposta à $\lambda$. Ou seja, se $\lambda=+,(1-\lambda)=\times$, e vice-versa. A equação que encontramos é

$$
\begin{aligned}
E_{\lambda}^{\prime \prime}+2 \mathcal{H} E_{\lambda}^{\prime}+k^{2} E_{\lambda}= & \sigma_{\mathrm{T} \lambda}\left[k^{2}\left(\frac{\Psi}{\mathcal{H}}\right)+X^{\prime}\right]+2 k^{2} \sum_{a, b} \mathcal{M}_{a b}^{\lambda} \tilde{\sigma}_{\mathrm{v} a} \Phi_{b} \\
& -2 k^{2} \sum_{a} \tilde{\sigma}_{\mathrm{v} a}^{2} E_{\lambda}-2 \sigma_{\mathrm{T} \times} \sigma_{\mathrm{T}+} E_{(1-\lambda)}+2 \sigma_{\mathrm{T}(1-\lambda)}^{2} E_{\lambda} .
\end{aligned}
$$

Novamente, no limite em que $\left(\sigma_{\|}, \sigma_{\mathrm{v} a}, \sigma_{\mathrm{T} \lambda}\right)=0$, temos a equação (3.70) dos modos de polarização das ondas gravitacionais num universo isotrópico.

\subsection{Equações reduzidas e variáveis de Mukhanov-Sasaki}

As equações (6.78-6.83, 6.86 e 6.87) formam um sistema de equações diferenciais acopladas para os modos escalares, vetoriais e tensoriais. Quando o universo é descrito pela métrica de FRW, os três tipos de perturbações se desacoplam e, por isso, podemos estudar a dinâmica de cada modo perturbativo de maneira independente. Evidentemente, esse procedimento não será possível no nosso caso. 
Introduziremos agora as variáveis de Mukhanov-Sasaki [56, 61] para os modos escalares e tensoriais

$$
v \equiv S Q, \quad \text { e } \quad \sqrt{\kappa} \mu_{\lambda} \equiv S E_{\lambda}, \quad \lambda=(+, \times)
$$

respectivamente, de modo idêntico ao que é feito em FRW, onde sabemos que estas três variáveis correspondem aos graus de liberdade canônicos utilizados na quantização do inflaton e das ondas gravitacionais primordiais (veja a discussão do §4).

\subsubsection{Modos escalares}

Comecemos introduzindo as variáveis (6.88) em nosso sistema de equações. Em seguida, notemos que a equação (6.80) pode ser reescrita numa forma mais compacta

$$
\left(2 \mathcal{H}-\sigma_{\|}\right) X=\frac{\kappa}{S} \varphi^{\prime} v+\sum_{\lambda} \sigma_{\mathrm{T} \lambda} E_{\lambda} .
$$

Combinando agora as equações (6.78) e (6.82), substituindo o modo vetorial pelo vínculo (6.85) e usando as equações de fundo (5.27-5.29), obtemos

$$
\mathcal{H} X^{\prime}+2\left(\mathcal{H}^{\prime}+2 \mathcal{H}^{2}\right) X+\kappa S V,_{\varphi} v+k^{2} \Psi=\frac{k^{2}}{3}(\Phi-\Psi)+\frac{2}{3} \frac{k^{2}}{\mathcal{H}} \sigma_{\|} \Psi
$$

Em seguida, substituímos $k^{2}(\Phi-\Psi)$ por sua expressão (6.83) e usamos novamente o vínculo (6.85). Encontramos então

$$
\left(2 \mathcal{H}-\sigma_{\|}\right)\left(X^{\prime}+\frac{k^{2}}{\mathcal{H}} \Psi\right)+4 \kappa S^{2} V X+2 \kappa S V,,_{\varphi} v=4 k^{2}\left(\sum_{a} \tilde{\sigma}_{\mathrm{v} a}^{2} X-\sum_{a, b, \lambda} \mathcal{M}_{a b}^{\lambda} \tilde{\sigma}_{\mathrm{v} a} \tilde{\sigma}_{\mathrm{v} b} E_{\lambda}\right) .
$$

O próximo passo consiste em substituir $Q$, definido em (6.60), na equação de KleinGordon (6.76), usando também a equação (5.30), para obtermos

$$
Q^{\prime \prime}+2 \mathcal{H} Q+k^{2} Q+S^{2} V,_{\varphi \varphi} Q+2 S^{2} V,_{\varphi} X-\varphi^{\prime}\left(X^{\prime}+\frac{k^{2}}{\mathcal{H}} \Psi\right)=0 .
$$

Agora substituímos $X$ e $\left(X^{\prime}+k^{2} \Psi / \mathcal{H}\right)$ por (6.89) e (6.91), respectivamente, obtendo assim

$$
\begin{aligned}
& Q^{\prime \prime}+2 \mathcal{H} Q+k^{2} Q+S^{2} V_{, \varphi \varphi} Q+2 S^{2} V_{, \varphi} X= \\
& \frac{\varphi^{\prime}}{\left(2 \mathcal{H}-\sigma_{\|}\right)}\left[4 k^{2}\left(\sum_{a} \tilde{\sigma}_{\mathrm{v} a}^{2} X-\sum_{a, b, \lambda} \mathcal{M}_{a b}^{\lambda} \tilde{\sigma}_{\mathrm{v} a} \tilde{\sigma}_{\mathrm{v} b} E_{\lambda}\right)-4 \kappa S^{2} V X-2 \kappa S V_{, \varphi} v\right] .
\end{aligned}
$$

Finalmente, introduzindo $v=S Q$ e utilizando as equações (6.67-6.69) obtemos, após um pouco de álgebra

$$
v^{\prime \prime}+\left(k^{2}-\frac{S^{\prime \prime}}{S}+S^{2} V_{, \varphi \varphi}\right) v=\frac{1}{S^{2}}\left(\frac{2 S^{2} \varphi^{2}}{2 \mathcal{H}-\sigma_{\|}}\right)^{\prime} \kappa v+\sum_{\nu} \frac{1}{S^{2}}\left(\frac{2 S^{2} \varphi^{\prime} \sigma_{\mathrm{T} \nu}}{2 \mathcal{H}-\sigma_{\|}}\right)^{\prime} \sqrt{\kappa} \mu_{\nu} .
$$

Essa equação é o primeiro resultado central deste trabalho. É importante enfatizar também a importância da relação (6.71) na dedução da equação (6.94). 


\subsubsection{Modos vetoriais}

Como vimos, os modos vetoriais contribuem apenas com o vínculo (6.85). Isso mostra que podemos utilizar esse vínculo para substituir os modos vetoriais do nosso sistema de equações pelos modos escalares e vetoriais.

\subsubsection{Modos tensoriais}

Substituindo a expressão (6.91) em (6.87) e fazendo a troca de variáveis $\sqrt{\kappa} \mu_{\lambda}=S E_{\lambda}$ encontramos a equação para os modos tensoriais

$$
\begin{aligned}
\mu_{\lambda}^{\prime \prime}+\left(k^{2}-\frac{S^{\prime \prime}}{S}\right) \mu_{\lambda}= & -2 \mu_{(1-\lambda)} \sigma_{\mathrm{T}+} \sigma_{\mathrm{T} \times}+2 \mu_{\lambda} \sigma_{\mathrm{T}(1-\lambda)}^{2}+\frac{1}{S^{2}}\left(\frac{2 S^{2} \varphi^{\prime} \sigma_{\mathrm{T} \lambda}}{2 \mathcal{H}-\sigma_{\|}}\right)^{\prime} \sqrt{\kappa} v \\
& +\sum_{\nu} \frac{1}{S^{2}}\left(\frac{2 S^{2} \sigma_{\mathrm{T} \nu} \sigma_{\mathrm{T} \lambda}}{2 \mathcal{H}-\sigma_{\|}}\right)^{\prime} \mu_{\nu}+\frac{\left(S^{2} \sigma_{\|}\right)^{\prime}}{S^{2}} \mu_{\lambda} .
\end{aligned}
$$

Esta equação é o segundo resultado central deste trabalho. Em particular, notemos que a condição de positividade da energia, implícita em $\sigma_{\|}<2 \mathcal{H}$, garante a estabilidade das equações durante o regime inflacionário.

\subsubsection{Equações reduzidas}

Graças ao vínculo (6.85) mostramos que é possível construir um sistema reduzido de equações de movimento envolvendo apenas três graus de liberdade, $\left(v, \mu_{+}, \mu_{\times}\right)$. Para deixar as equações numa forma mais compacta, definiremos quatro novas funções

$$
\begin{aligned}
& \frac{z_{\mathrm{s}}^{\prime \prime}}{z_{\mathrm{s}}}\left(\eta, k_{i}\right) \equiv \frac{S^{\prime \prime}}{S}-S^{2} V_{, \varphi \varphi}+\frac{1}{S^{2}}\left(\frac{2 S^{2} \kappa \varphi^{\prime 2}}{2 \mathcal{H}-\sigma_{\|}}\right)^{\prime}, \\
& \frac{z_{\lambda}^{\prime \prime}}{z_{\lambda}}\left(\eta, k_{i}\right) \equiv \frac{S^{\prime \prime}}{S}+2 \sigma_{\mathrm{T}(1-\lambda)}^{2}+\frac{1}{S^{2}}\left(S^{2} \sigma_{\|}\right)^{\prime}+\frac{1}{S^{2}}\left(\frac{2 S^{2} \sigma_{\mathrm{T} \lambda}^{2}}{2 \mathcal{H}-\sigma_{\|}}\right)^{\prime},
\end{aligned}
$$

$\mathrm{e}$

$$
\begin{aligned}
\aleph_{\lambda} & \equiv \frac{\sqrt{\kappa}}{S^{2}}\left(\frac{2 S^{2} \varphi^{\prime} \sigma_{\mathrm{T} \lambda}}{2 \mathcal{H}-\sigma_{\|}}\right)^{\prime}, \\
\beth & \equiv\left[\frac{1}{S^{2}}\left(\frac{2 S^{2} \sigma_{\mathrm{T} \times} \sigma_{\mathrm{T}+}}{2 \mathcal{H}-\sigma_{\|}}\right)^{\prime}-2 \sigma_{\mathrm{T} \times} \sigma_{\mathrm{T}}+\right] .
\end{aligned}
$$

Assim o sistema de equações se reduz a

$$
\begin{aligned}
v^{\prime \prime}+\left(k^{2}-\frac{z_{\mathrm{s}}^{\prime \prime}}{z_{\mathrm{s}}}\right) v & =\sum_{\nu} \aleph_{\nu} \mu_{\nu}, \\
\mu_{\lambda}^{\prime \prime}+\left(k^{2}-\frac{z_{\lambda}^{\prime \prime}}{z_{\lambda}}\right) \mu_{\lambda} & =\aleph_{\lambda} v+\beth \mu_{(1-\lambda)} .
\end{aligned}
$$

$\mathrm{Ou}$, numa forma mais compacta,

$$
\left(\begin{array}{c}
v \\
\mu_{+} \\
\mu_{\times}
\end{array}\right)^{\prime \prime}+\left(\begin{array}{ccc}
\omega_{\mathrm{s}}^{2} & -\aleph_{+} & -\aleph_{\times} \\
-\aleph_{+} & \omega_{+}^{2} & -\beth \\
-\aleph_{\times} & -\beth & \omega_{\times}^{2}
\end{array}\right)\left(\begin{array}{c}
v \\
\mu_{+} \\
\mu_{\times}
\end{array}\right)=0
$$


onde

$$
\omega_{\mathrm{s}}^{2}=k^{2}-\frac{z_{\mathrm{s}}^{\prime \prime}}{z}, \quad \omega_{\lambda}^{2}=k^{2}-\frac{z_{\lambda}^{\prime \prime}}{z} .
$$

Essa equação vetorial conclui o primeiro objetivo deste trabalho: encontrar as equações dinâmicas das perturbações dos modos escalares, vetoriais e tensoriais num universo do tipo Bianchi I.

$\mathrm{Na}$ ausência de cisalhamento do espaço, as equações se desacoplam e nós recuperamos as equações usuais (6.100) e (6.101) para as variáveis $v$ e $\mu_{\lambda}$ (4.15-4.16) [50]. No nosso caso, a anisotropia do espaço nos leva a alguns efeitos potencialmente interessantes. Primeiro, notemos que as funções $z_{s}$ e $z_{\lambda}$ não são apenas funções do tempo, mas dependem de $k_{i}$ através dos componentes do tensor de cisalhamento. Segundo, os dois tipo de modos (escalar e tensorial) estão acoplados por uma "matriz de massa" não-diagonal. Essa matriz de massa não pode ser diagonalizada simultaneamente com os modos perturbativos, o que nos leva a crer que esse sistema possui um mecanismo não-trivial de oscilação. A importância dos modos vetoriais na dedução dessas equações deve ser enfatizada. Se tivéssemos desprezado os mesmos a expressão para a matriz de massa estaria errada.

\subsection{Limite de pequenos comprimentos de onda}

Consideraremos agora o comportamento das equações (6.100) e (6.101) no limite em que os modos perturbativos estão dentro do horizonte de Hubble, ou seja, quando $k / \mathcal{H} \gg 1$. Para tanto, nos focaremos no comportamento das funções $\aleph_{\lambda}, \beth, z_{s}^{\prime \prime} / z_{s}$ e $z_{\lambda}^{\prime \prime} / z_{\lambda}$ no limite de pequenos comprimentos de onda. Da definição da função $\aleph_{\lambda}$ temos que

$$
\left|\aleph_{\lambda}\right| \leq\left|\frac{1}{S^{2}}\left(S^{2} \sigma_{\mathrm{T} \lambda}\right)^{\prime}\right| \times\left|\frac{2 \sqrt{\kappa} \varphi^{\prime}}{2 \mathcal{H}-\sigma_{\|}}\right|+2\left|\sigma_{\mathrm{T} \lambda}\right| \times\left|\left(\frac{\sqrt{\kappa} \varphi^{\prime}}{2 \mathcal{H}-\sigma_{\|}}\right)^{\prime}\right| .
$$

Notemos que a propriedade (6.74) implica que o primeiro termo do lado direito dessa desigualdade é menor que

$$
3 \sqrt{2} \mathcal{H}^{2}\left|\frac{2 \sqrt{\kappa} \varphi^{\prime}}{2 \mathcal{H}-\sigma_{\|}}\right|
$$

Logo,

$$
\left|\frac{2 \sqrt{\kappa} \varphi^{\prime}}{2 \mathcal{H}-\sigma_{\|}}\right|=\sqrt{2 \epsilon} \frac{\sqrt{\mathcal{H}^{2}-\frac{\sigma^{2}}{6}}}{\mathcal{H}-\frac{\sigma_{\|}}{2}} \leq \sqrt{2 \epsilon} \sqrt{\frac{1+x}{1-x}} .
$$

onde usamos o fato de que, dada a desigualdade $\sigma_{\|} / 2<\sigma / \sqrt{6}$ (veja a identidade (6.63)), deve existir um número $\alpha$ tal que $0 \leq x<\alpha$, onde o parâmetro de cisalhamento reduzido, $x$, foi introduzido em (5.38). Da desigualdade para $x$ deduzimos que $\sqrt{(1+x) / 1-x} \leq \sqrt{(1+\alpha) / 1-\alpha}$. A equação (6.72) então implica que o segundo termo da desigualdade (6.104) é menor que

$$
2 \sqrt{6} \mathcal{H} \times\left|\left(\frac{\sqrt{\kappa} \varphi^{\prime}}{2 \mathcal{H}-\sigma_{\|}}\right)^{\prime}\right|
$$


Logo, o valor absoluto nesta última expressão é limitado por

$$
\left|\left(\frac{\sqrt{\kappa} \varphi^{\prime}}{2 \mathcal{H}-\sigma_{\|}}\right)^{\prime}\right|<\left|\frac{\sqrt{\kappa} \varphi^{\prime \prime}}{2 \mathcal{H}-\sigma_{\|}}\right|+\left|\frac{\sqrt{\kappa} \varphi^{\prime}}{2 \mathcal{H}-\sigma_{\|}}\right|\left|\frac{2 \mathcal{H}^{\prime}-\sigma_{\|}^{\prime}}{2 \mathcal{H}-\sigma_{\|}}\right| .
$$

Agora, notemos que a equação (6.71) implica que $\left|\sigma_{\|}^{\prime}\right|<6 \mathcal{H}^{2}+\left|2 \mathcal{H} \sigma_{\|}\right|<10 \mathcal{H}^{2}$. Portanto

$$
\left|\left(\frac{\sqrt{\kappa} \varphi^{\prime}}{2 \mathcal{H}-\sigma_{\|}}\right)^{\prime}\right| \leq \sqrt{\frac{\epsilon}{2}} \mathcal{H} \frac{\sqrt{1+\alpha}}{\sqrt{1-\alpha}}\left[(1-\delta)+\frac{2(1-\epsilon)+\left(1-\frac{\epsilon}{3}\right) \sqrt{6}+10}{2(1-\alpha)}\right] .
$$

Colecionando todos os termos, concluímos que

$$
\left|\aleph_{\lambda}\right|<\sqrt{\epsilon} \mathcal{H}^{2} \sqrt{\frac{1+\alpha}{1-\alpha}}\left[6+2 \sqrt{3}\left((1-\delta)+\frac{2(1-\epsilon)+\left(1-\frac{\epsilon}{3}\right) \sqrt{6}+10}{2(1-\alpha)}\right)\right] .
$$

Nesse limite, portanto, vale a desigualdade $\left|\aleph_{\lambda}\right|<Z \mathcal{H}^{2}$, onde $Z$ é uma constante real. Em princípio, essa constante pode ser muito grande dado que o valor de $\alpha$ pode estar arbitrariamente perto de 1 quando a condição de positividade da energia estiver próxima do seu limiar de validade. Além disso, um valor grande de $Z$ também corresponde a um horizonte bastante elíptico, o que explica porque devemos tomar o limite em que $(k / \mathcal{H})^{2} \gg Z$.

$\mathrm{O}$ mesmo tipo de raciocínio empregado à função $\aleph_{\lambda}$ pode ser aplicado às funções $\beth, z_{s}^{\prime \prime} / z_{s}$ e $z_{\lambda}^{\prime \prime} / z_{\lambda}$. Concluímos, portanto, que no limite de pequenos comprimentos de onda $(k / \mathcal{H} \gg 1)$, os três graus de liberdade se desacoplam e passam a se comportar como um sistema de três osciladores harmônicos independentes, ou seja

$$
V^{\prime \prime}+k^{2} V=0, \quad V=\left(v, \mu_{+}, \mu_{\times}\right) .
$$

\subsection{Equações de perturbação a partir da ação}

Nesta seção nos dedicaremos a construir uma ação quadrática nas perturbações dos campos tal que, quando submetida ao princípio de mínima ação, nos forneça as equações de movimento $(6.100,6.101)$. Essa abordagem, além de servir como uma verificação independente da validade dos nossos cálculos, é de extrema importância para a construção das variáveis canônicas que, por sua vez, nos permitirão quantizar e normalizar os graus de liberdade do sistema.

\subsubsection{Formalismo ADM}

A construção da ação das perturbações cosmológicas é feita através do formalismo ADM, onde os vínculos entre as variáveis podem ser convenientemente eliminados por meio dos multiplicadores de Lagrange. O procedimento geral é o mesmo que foi resumido em §4.1. Inicialmente comparamos a métrica (6.29) no calibre newtoniano com a métrica (4.5). Trabalhando no tempo físico $\mathrm{d} t=\mathrm{d} \eta / S$, concluímos que a função lapso e o vetor deslocamento são dados respectivamente por

$$
N^{2}=(1+2 \Phi), \quad N_{i}=0
$$


e que a métrica $g_{i j}$, parametrizada na forma (6.46), é igual a

$$
g_{i j}=S^{2}(t)\left[\gamma_{i j}-2 \Psi\left(\gamma_{i j}+\frac{\hat{\sigma}_{i j}}{H}\right)+2 \partial_{(i} E_{j)}+2 E_{i j}\right] .
$$

Daí segue que os vínculos de energia e momento, em primeira ordem nas perturbações, ficam determinados respectivamente por

$$
\begin{aligned}
\frac{2}{S^{2}} \nabla^{2} \Psi-\frac{1}{S^{2}} \hat{\sigma}^{i j} \partial_{i} \partial_{j}\left(\frac{\Psi}{H}\right)-6 H \dot{\Psi}+ & \left(\frac{\Psi}{H}\right)^{\cdot} \hat{\sigma}^{2}-3 \Psi \hat{\sigma}^{2}-\Phi\left(6 H^{2}-\hat{\sigma}^{2}-\dot{\varphi}^{2}\right) \\
& +\frac{1}{S} \hat{\sigma}_{j}^{i} \partial_{i} \Phi^{j}-\hat{\sigma}_{j}^{i}\left(E_{j}^{i}\right)-\kappa V,_{\varphi} \delta \varphi-\kappa \dot{\varphi} \delta \dot{\varphi}=0
\end{aligned}
$$

$\mathrm{e}$

$$
\begin{aligned}
\hat{\sigma}^{2} \partial_{i}\left(\frac{\Psi}{H}\right)-\hat{\sigma}_{i}^{j} \partial_{j}\left[\Phi+\left(\frac{\Psi}{H}\right)^{\cdot}\right] & +2 \partial_{i}(\dot{\Psi}+H \Phi)-\frac{1}{2 S} \nabla^{2} \Phi_{i} \\
+ & +2 \hat{\sigma}^{j l} \partial_{j} E_{i l}-\hat{\sigma}^{j l} \partial_{i} E_{j l}-\kappa \dot{\varphi} \partial_{i} \delta \varphi=0
\end{aligned}
$$

onde passamos a indicar a derivada no tempo físico por um ponto e utilizamos a definição $\hat{\sigma} \equiv \sigma / S$. Uma vez escritas no espaço de Fourier e no tempo conforme $\mathrm{d} \eta=\mathrm{d} t / S$, a equação (6.114) fornece a equação (6.78) e a equação (6.115), projetada sobre seu modo escalar, fornece (6.80).

Para expandir a ação até segunda ordem em todas as variáveis perturbadas, reescreveremos a métrica espacial $g_{i j}$ como

$$
g_{i j}=S^{2}\left(\gamma_{i j}+h_{i j}\right),
$$

sendo $h_{i j}$ a métrica definida em (6.46) escrita agora no tempo físico. A métrica inversa $g^{i j}$ e seu determinante são dados respectivamente por

$$
g^{i j}=S^{-2}\left(\gamma^{i j}-h^{i j}+h^{i l} h_{l}^{j}\right), \quad \sqrt{g}=S^{3}\left[1+\frac{1}{2} h+\frac{1}{8} h^{2}-\frac{1}{4} h_{j}^{i} h_{i}^{j}\right] .
$$

O procedimento geral de construção da ação quadrática consiste em substituir as expressões $(6.57,6.112,6.117$, e 6.118) na ação (4.6) e expandí-la até a segunda ordem nas variáveis perturbativas. Nesse processo, encontraremos termos de zero, primeira e segunda ordens nas perturbações. Os termos de ordem zero e de primeira ordem são

$$
\begin{aligned}
S_{0}= & \frac{1}{2 \kappa} \int \mathrm{d} t \mathrm{~d}^{3} x\left[S^{3}\left(-6 H^{2}+\hat{\sigma}^{2}-2 \kappa V+\kappa \dot{\varphi}^{2}\right)\right] \\
S_{1}= & \frac{1}{2 \kappa} \int \mathrm{d} t \mathrm{~d}^{3} x S^{3}\left[R_{1}^{(3)}+\hat{\sigma}^{i j} \dot{h}_{i j}-2 \hat{\sigma}_{i j} \hat{\sigma}_{l}^{i} h^{j l}+12 H \dot{\Psi}+3 \Psi\left(6 H^{2}-\hat{\sigma}^{2}+2 \kappa V-\kappa \dot{\varphi}^{2}\right)\right. \\
& \left.+\Phi\left(6 H^{2}-\hat{\sigma}^{2}-2 \kappa V-\kappa \dot{\varphi}^{2}\right)-2 \kappa V{ }_{, \varphi} \delta \varphi+2 \kappa \dot{\varphi} \delta \dot{\varphi}\right] .
\end{aligned}
$$

Utilizando as equações (5.27-5.29) e com um pouco de álgebra, podemos mostrar que estes termos se reduzem a derivadas totais

$$
\begin{aligned}
S_{0}= & \frac{1}{2 \kappa} \int \mathrm{d} t \mathrm{~d}^{3} x\left[-4 \frac{\mathrm{d}}{\mathrm{d} t}\left(S^{3} H\right)\right] \\
S_{1}= & \frac{1}{2 \kappa} \int \mathrm{d} t \mathrm{~d}^{3} x\left\{\partial_{i}\left[\partial^{i}(4 S \Psi)-\partial^{i}\left(\frac{S \Psi}{H}\right)\right]\right. \\
& \left.+\frac{\mathrm{d}}{\mathrm{d} t}\left[\nabla^{2}\left(\frac{S \Psi}{H}\right)+S^{3} \hat{\sigma}^{i j} h_{i j}+12 S^{3} H \Psi+2 S^{3} \kappa \dot{\varphi} \delta \varphi\right]\right\}
\end{aligned}
$$


e, sendo assim, os mesmos não contribuem para a dinâmica das equações de movimento. A primeira contribuição não-trivial resulta dos termos de segunda ordem na ação

$$
\begin{aligned}
S_{2}= & \frac{1}{2 \kappa} \int \mathrm{d} t \mathrm{~d}^{3} x S^{3}\left[R_{2}^{(3)}+N_{1} R_{1}^{(3)}+\frac{1}{2} h R_{1}^{(3)}+\mathcal{K}_{2}+\frac{1}{2} h \mathcal{K}_{1}+\frac{1}{8} h^{2} \mathcal{K}_{0}\right. \\
& -\frac{1}{4} h_{j}^{i} h_{i}^{j} \mathcal{K}_{0}-N_{1} \mathcal{K}_{1}-\frac{1}{2} N_{1} h \mathcal{K}_{0}+N_{1}^{2} \mathcal{K}_{0}+\kappa\left(-S^{-2} \partial_{i} \delta \varphi \partial^{i} \delta \varphi-V,_{\varphi \varphi} \delta \varphi^{2}\right. \\
& -2 N_{1} V,_{\varphi} \delta \varphi-h V,_{\varphi} \delta \varphi-h N_{1} V-\frac{1}{4} h^{2} V+\frac{1}{2} h_{j}^{i} h_{i}^{j} V+\delta \dot{\varphi}^{2}-2 N_{1} \dot{\varphi} \delta \dot{\varphi} \\
& \left.\left.+N_{1}^{2} \dot{\varphi}^{2}+h \dot{\varphi} \delta \dot{\varphi}-\frac{1}{2} h N_{1} \dot{\varphi}^{2}+\frac{1}{8} h^{2} \dot{\varphi}^{2}-\frac{1}{4} h_{j}^{i} h_{i}^{j} \dot{\varphi}^{2}\right)\right]
\end{aligned}
$$

na qual

$$
\begin{aligned}
S^{2} R_{1}^{(3)} \equiv & 4\left(\nabla^{2}-\frac{\hat{\sigma}^{i j} \partial_{i} \partial_{j}}{2 H}\right) \Psi \\
S^{2} R_{2}^{(3)} \equiv & -\partial_{l} h^{l j} \partial_{i} h_{j}^{i}-2 h^{j l} \partial_{j} \partial_{i} h_{l}^{i}-9 \partial_{i} \Psi \partial^{i} \Psi-\frac{1}{4} \partial_{l} h^{i j} \partial^{l} h_{i j}-\frac{1}{2} \partial_{l} h_{i j} \partial^{i} h^{l j} \\
& -6 \partial_{i}\left(h^{j i} \partial_{j} \Psi\right)+\frac{1}{2} \partial^{i} \partial_{i}\left(h^{j l} h_{j l}\right) \\
\mathcal{K}_{0} \equiv & -6 H^{2}+\hat{\sigma}^{2} \\
\mathcal{K}_{1} \equiv & -2 H \dot{h}+\hat{\sigma}^{i j} \dot{h}_{i j}-2 \hat{\sigma}_{i j} \hat{\sigma}_{l}^{j} h^{l i} \\
\mathcal{K}_{2} \equiv & 2 H \dot{h}_{i j} h^{i j}-4 H \hat{\sigma}_{i j} h^{i l} h_{l}^{j}-2 \hat{\sigma}_{i}^{l} h^{i m} \dot{h}_{m l}+2 \hat{\sigma}_{i j} \hat{\sigma}_{l}^{j} h^{i m} h_{m}^{l}+\frac{1}{4} \dot{h}^{i j} \dot{h}_{i j} \\
& +\hat{\sigma}_{i j} \hat{\sigma}_{l m} h^{i m} h^{j l}-\frac{1}{4} \dot{h}^{2} .
\end{aligned}
$$

O processo rigoroso de redução da ação (6.123) a uma forma canônica, que faremos utilizando o tempo conforme $\eta$, deve ser desenvolvido no espaço de Fourier, uma vez que vários operadores não-locais, tais como o laplaciano inverso $\nabla^{-2}$ e operadores da forma $\left(\sigma_{i j} \partial^{i} \partial^{i}\right)^{-1}$, surgem quando utilizamos os vínculos $(6.114,6.115)$ para reduzir o número de graus de liberdade espúrios. Além disso, as operações no espaço de Fourier facilitam o uso das equações (6.67-6.69), definidas apenas em termos dos modos $k$. Uma vez no espaço de Fourier, as derivadas espaciais totais são identicamente nulas. Por exemplo, uma derivada total do tipo $\partial_{l}\left(\Psi \partial^{l} \Psi\right)$ no espaço de Fourier fica

$$
\int \mathrm{d} \eta \mathrm{d}^{3} x \partial_{l}\left(\Psi \partial^{l} \Psi\right)=\int \mathrm{d} \eta \mathrm{d}^{3} k \mathrm{~d}^{3} q\left[-\boldsymbol{k} \cdot(\boldsymbol{k}+\boldsymbol{q}) \Psi_{\boldsymbol{k}} \Psi_{\boldsymbol{q}}\right] \delta^{(3)}(\boldsymbol{k}+\boldsymbol{q})=0 .
$$

O próximo passo consiste em substituir $h_{i j}$ por sua expressão (6.46). Quando isso é feito, os termos envolvendo $E_{j}$ ou se anulam, ou têm a forma $\left(E_{j}\right)^{\prime}=-\Phi_{j}$ (no calibre newtoniano). Em seguida, decompomos $\Phi_{j}$ e $E_{i j}$ na forma (6.9) e (6.14). O vínculo (6.115), uma vez reescrito no espaço de Fourier e projetado sobre seus modos escalar e vetoriais, fornece as equações (6.89) e (6.85). Essas equações são então reinseridas na ação, que fica agora determinada em termos das variáveis $(X, \Phi, \Psi)$ e $\left(\delta \varphi, v, E_{\lambda}\right)$. 
Eliminando as variáveis $(X, \Phi, \Psi)$ em termos da expressão (6.79) e substituindo $\delta \varphi$ e $E_{\lambda}$ em termos das variáveis $v$ e $\mu_{\lambda}$ chegamos, finalmente, à forma canônica da ação

$$
\begin{aligned}
S_{2}= & \frac{1}{2} \int \mathrm{d} \eta \mathrm{d}^{3} k\left\{v^{\prime} v^{\prime *}+\left(\frac{z_{\mathrm{s}}^{\prime \prime}}{z_{\mathrm{s}}}-k^{2}\right) v v^{*}+\sum_{\nu} \frac{1}{S^{2}}\left(\frac{2 S^{2} \sqrt{\kappa} \varphi^{\prime} \sigma_{\mathrm{T} \nu}}{2 \mathcal{H}-\sigma_{\|}}\right)\left(v^{*} \mu_{\nu}+v \mu_{\nu}^{*}\right)\right. \\
& \sum_{\lambda}\left[\mu_{\lambda}^{\prime} \mu_{\lambda}^{\prime *}+\left(\frac{z_{\lambda}^{\prime \prime}}{z_{\lambda}}-k^{2}\right) \mu_{\lambda} \mu_{\lambda}^{*}+\left[-2 \sigma_{\mathrm{T}}+\sigma_{\mathrm{T} \times}+\frac{1}{S^{2}}\left(\frac{S^{2} \sigma_{\mathrm{T}} \sigma_{\mathrm{T} \times}}{2 \mathcal{H}-\sigma_{\|}}\right)^{\prime}\right] \mu_{(1-\lambda)} \mu_{\lambda}^{*}\right] \\
& +\mathcal{T}\}
\end{aligned}
$$

onde $\mathcal{T}$ é uma derivada temporal total (apresentada no Apêndice B). Fica evidente agora que a variação da ação canônica (6.130) com relação às variáveis $v^{*}$ e $\mu_{\lambda}^{*}$ fornece imediatamente as equações (6.94) e (6.95) e que $\left(v, \mu_{\lambda}\right)$ são as variáveis canônicas do sistema, às quais estão associados os momentos conjugados $\left(\pi_{v}, \pi_{\lambda}\right)=\left(v^{\prime *}, \mu_{\lambda}^{\prime *}\right)$. A ação acima deve ser comparada à sua análoga isotrópica (4.14). Tomando o limite apropriado de isotropia, $\sigma \rightarrow 0$, os modos escalares e tensoriais se desacoplam e a ação acima se reduz à (4.14). 


\section{CAPÍTULO 7}

\section{Quantização das Perturbações e Previsões}

Nos capítulos anteriores apresentamos uma descrição detalhada da dinâmica homogênea e perturbativa (em primeira ordem nas perturbações) de um universo inflacionário do tipo Bianchi I. Nosso principal objetivo neste capítulo é o de quantizar as perturbações cosmológicas descritas anteriormente e avaliar o impacto das peculiaridades do universo anisotrópico sobre os espectros de potências das perturbações. Para tanto, comecemos por relembrar que o sistema de equações (6.100-6.101), por si só, não determina completamente a evolução dinâmica dos três graus de liberdade $v, \mu_{+}$e $\mu_{\times}$. Qualquer previsão física depende também da especificação de um conjunto de condições iniciais para esses graus de liberdade. Em espaços-tempo de FRW, o procedimento de determinação das condições iniciais e da quantização dos graus de liberdade é bem conhecido [50] e depende crucialmente da existência de um regime onde os graus de liberdade evoluem adiabaticamente [59]. Precisamos portanto entender, no contexto de espaços-tempo anisotrópicos, quão robusto é esse procedimento frente a um componente não nulo de anisotropia espacial. Começaremos então revendo o procedimento padrão empregado em espaços isotrópicos procurando enfatizar suas hipóteses principais. Em seguida, em $\S 7.2$, iremos enfatizar e quantificar as diferenças inerentes aos dois espaços. Essa análise nos levará por fim $(\$ 7.3)$ a uma extensão do procedimento de quantização usual. Em §7.5, apresentaremos uma análise crítica do nosso procedimento, discutindo suas limitações e seus pontos fracos.

\subsection{Universos de FRW}

Por simplicidade, consideraremos aqui o caso de um campo sem massa, $\varphi=v / a$, evoluindo numa fase inflacionária de de Sitter pura (veja a discussão feita em $§ 2.3$ para mais detalhes). Essa hipótese simplificadora não representa nenhuma limitação aos argumentos seguintes, os quais podem ser facilmente generalizados para casos mais realistas de expansão "quase" de Sitter (veja a discussão em §4.2) 


\subsubsection{Quantização Canônica}

Para que possam ser quantizados, os graus de liberdade das perturbações cosmológicas são promovidos ao status de operadores quânticos e decompostos na forma

$$
\begin{aligned}
\hat{v}(\boldsymbol{x}, \eta) & \equiv \int \frac{\mathrm{d}^{3} k}{(2 \pi)^{3 / 2}}\left[\hat{v}_{\boldsymbol{k}}(\eta) e^{i \boldsymbol{k} \cdot \boldsymbol{x}}+\hat{v}_{\boldsymbol{k}}^{\dagger}(\eta) e^{-i \boldsymbol{k} \cdot \boldsymbol{x}}\right], \\
& =\int \frac{\mathrm{d}^{3} k}{(2 \pi)^{3 / 2}}\left[v_{k}(\eta) e^{i \boldsymbol{k} \cdot \boldsymbol{x}} \hat{a}_{\boldsymbol{k}}+v_{k}^{*}(\eta) e^{-i \boldsymbol{k} \cdot \boldsymbol{x}} \hat{a}_{\boldsymbol{k}}^{\dagger}\right] .
\end{aligned}
$$

Os operadores de criação e aniquilação, $\hat{a}_{\boldsymbol{k}}$ e $\hat{a}_{\boldsymbol{k}}^{\dagger}$, satisfazem as regras de comutação

$$
\left[\hat{a}_{\boldsymbol{k}}, \hat{a}_{\boldsymbol{q}}\right]=\left[\hat{a}_{\boldsymbol{k}}^{\dagger}, \hat{a}_{\boldsymbol{q}}^{\dagger}\right]=0, \quad\left[\hat{a}_{\boldsymbol{k}}, \hat{a}_{\boldsymbol{q}}^{\dagger}\right]=\delta^{(3)}(\boldsymbol{k}-\boldsymbol{q}) .
$$

Na decomposição (7.1), o modo perturbativo $v_{k}(\eta)$ é solução da equação clássica

$$
v_{k}^{\prime \prime}+\omega_{v}^{2}(k, \eta) v_{k}=0 \quad \text { com } \quad \omega_{v}^{2}(k, \eta)=k^{2}-\frac{2}{\eta^{2}},
$$

que, como podemos ver, nada mais é que a equação de um oscilador harmônico com uma massa dependente do tempo. A massa dinâmica, neste caso, é uma consequência imediata do fato do modo perturbativo viver em um espaço-tempo dinâmico. A equação (7.4) tem como solução geral

$$
v_{k}(\eta)=\left[A(k) H_{\nu}^{(1)}(-k \eta)+B(k) H_{\nu}^{(2)}(-k \eta)\right] \sqrt{-\eta}
$$

em que $H_{\nu}^{(1,2)}$ são as funções de Hankel de primeira e segunda espécies. No caso particular de uma fase puramente de Sitter considerada aqui, temos $\nu=3 / 2$ (veja a definição (2.60)), logo

$$
H_{3 / 2}^{(2)}(z)=\left[H_{3 / 2}^{(1)}(z)\right]^{*}=-\sqrt{\frac{2}{\pi z}} e^{-i z}\left(1+\frac{1}{i z}\right) .
$$

O procedimento de quantização canônica consiste em impor, em hipersuperfícies de tempo constante, as seguintes regras de comutação

$$
[\hat{v}(\boldsymbol{x}, \eta), \hat{v}(\boldsymbol{y}, \eta)]=[\hat{\pi}(\boldsymbol{x}, \eta), \hat{\pi}(\boldsymbol{y}, \eta)]=0 \quad \text { e } \quad[\hat{v}(\boldsymbol{x}, \eta), \hat{\pi}(\boldsymbol{y}, \eta)]=i \delta^{(3)}(\boldsymbol{x}-\boldsymbol{y})
$$

onde $\hat{\pi}$ é o operador de momento canonicamente conjugado à "variável de posição" $\hat{v}$. Utilizando a decomposição (7.1) juntamente com as regras de comutação dos operadores de criação e aniquilação, a segunda das equações (7.7) implica na seguinte condição de normalização dos modos $v_{k}$

$$
v_{k} v_{k}^{*}-v_{k}^{*} v_{k}^{\prime}=i
$$

o que também determina a normalização do Wronskiano das soluções. A escolha de um modo específico $v_{k}(\eta)$ corresponde à escolha do vácuo físico $|0\rangle$, definido por

$$
\hat{a}_{\boldsymbol{k}}|0\rangle=0
$$


A escolha mais "natural" para esse estado de vácuo corresponde à escolha de uma solução $v_{k}(\eta)$ que, em um determinado limite, convirja adiabaticamente ao modo de Minkowski, dado por

$$
v_{k} \rightarrow \frac{1}{\sqrt{2 k}} e^{-i k \eta}
$$

Como veremos adiante, no exemplo em questão esse limite é único e equivale a $k \eta \rightarrow$ $-\infty$. Munidos dessa prescrição e da condição de normalização do Wronskiano, a solução geral (7.5) fica determinada por

$$
v_{k}=\frac{1}{\sqrt{2 k}}\left(1+\frac{1}{i k \eta}\right) e^{-i k \eta} .
$$

O vácuo definido pela solução acima é também conhecido como vácuo de Bunch-Davies [59].

\subsubsection{Aproximação WKB}

No caso de espaços-tempo mais gerais, e certamente no caso de universos do tipo Bianchi I, uma solução exata para a equação (7.4) pode ser difícil, ou até mesmo impossível, de se encontrar. Em geral, esse problema é contornado por meio da aproximação WKB [92], cuja interpretação física está intimamente ligada à nossa compreensão de um estado de vácuo "bem definido" [59]. A aproximação WKB consiste em introduzir o seguinte ansatz para o modo perturbativo

$$
v_{k}^{\mathrm{WKB}}(\eta)=\frac{1}{\sqrt{2 \omega_{v}}} e^{ \pm i \int \omega_{v} \mathrm{~d} \eta}
$$

que satisfaz a equação

$$
v_{k}^{\mathrm{WKB} \prime \prime}+\left(\omega_{v}^{2}-Q_{\mathrm{WKB}}\right) v_{k}^{\mathrm{WKB}}=0
$$

sendo que

$$
Q_{\mathrm{WKB}} \equiv \frac{3}{4}\left(\frac{\omega_{v}^{\prime}}{\omega_{v}}\right)^{2}-\frac{1}{2} \frac{\omega_{v}^{\prime \prime}}{\omega_{v}} .
$$

É fácil ver, portanto, que para um modo que satisfaz a equação (7.4), a solução WKB será uma boa aproximação na medida em que a condição $\left|Q_{\mathrm{WKB}} / \omega_{v}^{2}\right| \ll 1$ for satisfeita. No caso particular da solução (7.11), esta condição se reduz a

$$
\left|\frac{Q_{\mathrm{WKB}}}{\omega_{v}^{2}}\right|=\left|\frac{1-3 k^{2} \eta^{2}}{\left(2-k^{2} \eta^{2}\right)^{3}}\right| \ll 1 .
$$

Dado que a variável temporal $\eta$ se restringe ao intervalo $\eta \in(-\infty, 0)$, a condição acima se reduz à $k \eta \rightarrow-\infty$, como esperado. Isso significa que, para modos com comprimentos de onda muito menores que o raio de Hubble, ou seja, $k \ll a H=-1 / \eta$, a solução (7.11) é uma boa aproximação da solução WKB. Em outras palavras, isso significa que a noção de um estado de vácuo adiabático está bem definida para estes modos. Esse aspecto do modelo é crucial, pois é sobre ele que reside o procedimento de quantização dos modos cosmológicos em universos isotrópicos. 


\subsubsection{Espectro de Potência em escalas de super-Horizonte}

Uma vez fixadas as condições iniciais, o modo $v_{k}$ fica completamente determinado e pode então ser relacionado ao campo escalar $\varphi$ (nesse caso, o inflaton, também promovido ao status de operador). Uma vez que os modos perturbativos se encontrem em escalas de super-horizonte, ou seja, $k \eta \ll 1$, o campo escalar $\varphi$ fica determinado por

$$
\hat{\varphi} \rightarrow \int \frac{\mathrm{d}^{3} k}{(2 \pi)^{3 / 2}} \hat{\varphi}_{\boldsymbol{k}} e^{i \boldsymbol{k} \cdot \boldsymbol{x}}=\int \frac{\mathrm{d}^{3} k}{(2 \pi)^{3 / 2}} \frac{H}{\sqrt{2 k^{3}}}\left(\hat{a}_{\boldsymbol{k}}+\hat{a}_{-\boldsymbol{k}}^{\dagger}\right) e^{i \boldsymbol{k} \cdot \boldsymbol{x}}
$$

onde usamos $S(\eta)=-1 / H \eta$ para uma fase inflacionária de de Sitter pura, caracterizada por $H=$ constante. Vemos que, neste limite, todos os operadores são proporcionais a $\left(\hat{a}_{\boldsymbol{k}}+\hat{a}_{-\boldsymbol{k}}^{\dagger}\right)$ e, portanto, todos os operadores comutam entre si. Isso nos leva a crer que o operador $\hat{\varphi}$ possui todas as propriedades de uma variável estocástica gaussiana e, dessa forma, o procedimento de quantização dos modos perturbativos será análogo ao que se obtém substituindo os operadores quânticos por campos estocásticos gaussianos. Nessa descrição, os modos perturbativos, que antes eram promovidos à operadores quânticos, são agora substituídos por variáveis estocásticas de tal modo que $\hat{v}_{\boldsymbol{k}} \rightarrow v_{\boldsymbol{k}}=v_{k}(\eta) e_{v}(\boldsymbol{k})$, onde a variável unitária e aleatória, $e_{v}(\boldsymbol{k})$, está sujeita as condições

$$
\left\langle e_{v}(\boldsymbol{k})\right\rangle=0, \quad\left\langle e_{v}(\boldsymbol{k}) e_{v}^{*}(\boldsymbol{q})\right\rangle=\delta^{(3)}(\boldsymbol{k}-\boldsymbol{q}) .
$$

e onde identificamos a média (quântica) no estado de vácuo, i.e. $\langle 0|\ldots| 0\rangle$, por uma média (clássica) em um ensemble, i. e. $\langle\ldots\rangle$.

A função de correlação associada ao modo $v_{k}$ é definida, na abordagem quântica usual, como

$$
\xi_{v} \equiv\langle 0|\hat{v}(\boldsymbol{x}, \eta) \hat{v}(\boldsymbol{y}, \eta)| 0\rangle,
$$

e toma a seguinte forma simples

$$
\xi_{v}=\int \frac{\mathrm{d}^{3} k}{(2 \pi)^{3}}\left|v_{k}\right|^{2} e^{i \boldsymbol{k} \cdot(\boldsymbol{x}-\boldsymbol{y})} .
$$

Neste ponto é interessante notar que, em um universo de FRW, a isotropia espacial nos permite integrar esta expressão sobre um ângulo sólido e assim obter

$$
\xi_{v}=\int \frac{\mathrm{d} k}{k} \frac{k^{3}}{2 \pi^{2}}\left|v_{k}\right|^{2} \frac{\sin k r}{k r}
$$

o que, graças às simetrias do espaço-tempo, significa dizer que $\xi_{v}$ é uma função de $r=|\boldsymbol{x}-\boldsymbol{y}|$ apenas. O espectro de potência é definido como

$$
P_{v}(k)=\left|v_{k}\right|^{2}, \quad \mathcal{P}_{v}(k)=\frac{k^{3}}{2 \pi^{2}}\left|v_{k}\right|^{2} .
$$

Na abordagem estocástica, a fórmula para a função de correlação do modo $v_{\boldsymbol{k}}$ é dada por

$$
\left\langle v_{\boldsymbol{k}} v_{\boldsymbol{q}}^{*}\right\rangle=P_{v}(k) \delta^{(3)}(\boldsymbol{k}-\boldsymbol{q}),
$$

a partir da qual se deduz o espectro de potência da variável de perturbação de curvatura $\mathcal{R}$

$$
P_{\mathcal{R}}(k)=\frac{2 \pi^{2}}{k^{3}} \mathcal{P}_{\mathcal{R}}(k)=\frac{\left|v_{k}\right|^{2}}{z^{2}} .
$$


Todo esse procedimento pode ser refeito para os graus de liberdade associados às polarizações das ondas gravitacionais. Uma vez que as mesmas não se acoplam ao modo escalar nem mesmo entre si, podemos introduzir dois conjuntos de operadores de criação e aniquilação, $\hat{b}_{\boldsymbol{k}, \lambda},(\lambda=+$ ou $\times$, dependendo da polarização). Em escalas muito maiores que o raio de Hubble, os dois modos podem ser descritos por duas variáveis estocásticas independentes, $\mu_{\boldsymbol{k}, \lambda} \equiv \mu_{\boldsymbol{k}} e_{\lambda}(\boldsymbol{k})$, onde novamente

$$
\left\langle e_{\lambda}(\boldsymbol{k}) e_{\lambda^{\prime}}^{*}(\boldsymbol{q})\right\rangle=\delta_{\lambda \lambda^{\prime}} \delta^{(3)}(\boldsymbol{k}-\boldsymbol{q}) .
$$

Os espectros de potência para ambos os modos ficam determinados por

$$
P_{\lambda}(k)=\frac{2 \pi^{2}}{k^{3}} \mathcal{P}_{\lambda}(k)=\left|\mu_{k}\right|^{2} .
$$

Novamente, a isotropia espacial implica que $P_{+}=P_{\times}$, de modo que o espectro de potência total das ondas gravitacionais é

$$
P_{T}(k)=2 \frac{\kappa}{S^{2}} P_{+}(k) .
$$

Concluindo, essa revisão do procedimento padrão de quantização enfatiza (1) a importância do regime WKB em escalas muito menores que o horizonte de Hubble, o qual permite construir um estado de vácuo adiabático bem definido, (2) o fato de que, em escalas maiores que o horizonte de Hubble (onde se pretende tirar conclusões físicas sobre o espectro de potência), os operadores quânticos podem ser convenientemente substituídos por campos estocásticos, (3) a importância da isotropia espacial que implica na existência de 3 direções espaciais independentes e (4) na semelhança do espectro de potência das ondas gravitacionais, compartilhado pelas duas polarizações $\lambda=+\mathrm{e} \lambda=\times$. Vejamos agora como cada um desses aspectos se comportam e se generalizam no caso dos universos de Bianchi I.

\subsection{Universos de Bianchi I}

\subsubsection{Número de onda característico}

Visando relacionar nossas previsões às observações, introduziremos um número de onda de referência, através de

$$
\left.k_{\text {ref }} \equiv S H\right|_{t=\tau_{*}}
$$

sendo que o parâmetro $\tau_{*}$ foi definido em (5.66). Em seguida, definiremos $\mathcal{N}$ como sendo o número de $e$-folds com a quantidade $S H$ ao invés de apenas $S$, como feito em (5.75). Chamando de $\mathcal{N}_{\text {ref }}$ o número de $e$-folds entre $t=\tau_{*}$ e o fim da inflação, e de $\mathcal{N}_{0}$ o número de $e$-folds associado ao período entre o fim da inflação e hoje, podemos

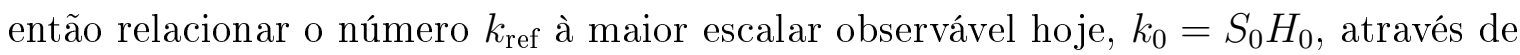

$$
\frac{k_{\text {ref }}}{k_{0}}=e^{\left(\mathcal{N}_{0}-\mathcal{N}_{\text {ref }}\right)} .
$$

Dado que na era inflacionária posterior a $\tau_{*}$ o parâmetro $H$ é aproximadamente constante, então $\mathcal{N}_{\text {ref }} \simeq N_{\text {ref }}$. Já o valor de $\mathcal{N}_{0}$ depende da evolução pós-inflacionária do 
universo e pode ser estimado como sendo [93, 94]

$$
\mathcal{N}_{0} \simeq 62-\ln \left(\frac{10^{16} \mathrm{GeV}}{V_{k_{0}}^{1 / 4}}\right)+\frac{1}{4} \ln \frac{V_{k_{0}}}{V_{\text {end }}}-\frac{1}{3} \ln \left(\frac{V_{\text {end }}^{1 / 4}}{\rho_{\text {reh }}^{1 / 4}}\right)-\ln h,
$$

onde $h$ é o parâmetro de Hubble em unidades de $100 \mathrm{~km} \cdot \mathrm{s}^{-1} / \mathrm{Mpc}, \rho_{\text {reh }}$ é a energia da fase de reaquecimento do universo após a inflação e $V_{\text {end }}$ o valor do potencial no fim da inflação. Tipicamente teremos $V_{k_{0}} \sim V_{\text {end }}$ enquanto for válida a aproximação de rolagem lenta. Com relação à temperatura de reaquecimento, argumenta-se geralmente que essa deva ser maior que $\rho_{\text {reh }}^{1 / 4}>10^{10} \mathrm{GeV}$ para se evitar o problema dos gravitinos [95]. A amplitude das flutuações cosmológicas, (tipicamente da ordem de $10^{-5} \mathrm{em}$ escalas de super-horizonte) implica que $V_{\text {end }}^{1 / 4}$ é menor que $10^{16} \mathrm{GeV}$ e, pelos mesmos motivos descritos acima, deve ser maior que $10^{10} \mathrm{GeV}$ nos casos extremos. Isso implica que o valor de $\mathcal{N}_{0}$ deve estar aproximadamente entre 50 e 70 , o que está de acordo com o valor necessário para se solucionar os problemas do horizonte e da planura do universo.

O valor de $N_{\text {ref }}$ pode ser calculado a partir da dinâmica homogênea do universo e, como mostrado na Fig. 5.7, é aproximadamente equivalente ao valor obtido no caso isotrópico.

\subsubsection{Anisotropia}

A primeira diferença óbvia com o caso de FRW decorre da anisotropia local do espaço. Em primeiro lugar, fica claro a partir do conjunto de equações $(6.100,6.101)$ que, devido à anisotropia do espaço, a evolução de um modo perturbativo qualquer dependerá não apenas do módulo do número de onda $k$ mas também de sua direção, $k_{i}$. Essa violação explicita de anisotropia se refletirá nos seguintes fatos

1. Os espectros de potências no fim da inflação serão funções de $\boldsymbol{k}$ e não de $k$, i.e., $P_{v}=P_{v}(\boldsymbol{k})$ e $P_{\lambda}=P_{\lambda}(\boldsymbol{k})$

2. Devido ao acoplamento entre o modo escalar e as ondas gravitacionais, existe uma correlação cruzada e não-nula entre esses modos, ou seja, $\left\langle v \mu_{\lambda}\right\rangle \neq 0$;

3. As duas polarizações das ondas gravitacionais devem ter, a priori, dois espectros de potências diferentes, i.e. $P_{+} \neq P_{\times}$. 


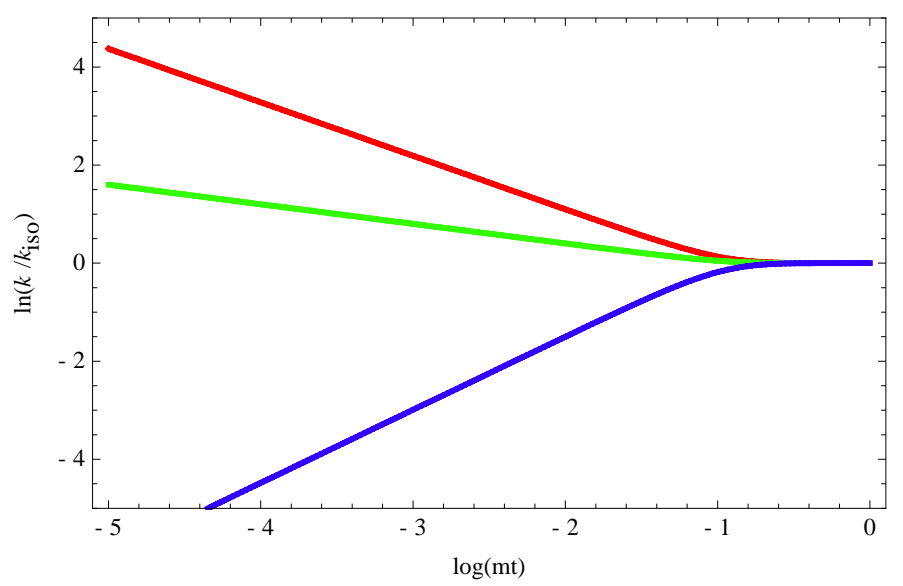

Figura 7.1: Logaritmo da razão $k / k_{\text {iso }}$, onde $k_{\text {iso }}$ é o módulo do mesmo número de onda quando considerado em um universo isotrópico. Olhando no sentido decrescente do tempo vemos que, à medida que o cisalhamento cresce, o modo $k$ passa a depender da direção espacial à qual está alinhado (cada cor corresponde a um dos eixos 3 ortogonais $x, y$ e $z$ ). A figura acima refere-se a um modelo específico com $\alpha=\pi / 4$.

Existe ainda um quarto aspecto importante que surge como conseqüência da evolução de um dado número de onda comóvel. Para ilustrar esse ponto consideremos as diferentes evoluções no tempo de um número de onda $k$ de acordo com sua orientação espacial. Como nos mostra a Fig. 7.1, a evolução inicial deste modo depende explicitamente da sua orientação com um dos eixos ortogonais $x, y$ ou $z$. À medida que o universo se isotropiza essa dependência desaparece e os três valores de $k$ convergem para um mesmo número.

\subsubsection{Aproximação WKB}

Qualquer que seja o parâmetro $\alpha$, definido em (5.35), que caracteriza o universo Bianchi I, sempre existirá um período pré-inflacionário de domínio do cisalhamento. Durante essa fase, a condição $\ddot{S}<0$ é satisfeita. Além disso, em um espaço-tempo do tipo Bianchi I genérico ${ }^{1}$, dois dos três fatores de escala crescem com o tempo enquanto o terceiro sofre um ricochete ${ }^{2}$.

Para procedermos com a análise da aproximação WKB usaremos a razão $k / S H$ (como é usualmente feito) para discutir se um dado modo $k$ está dentro $(k / S H \gg 1$, regime de sub-horizonte) ou fora $(k / S H \ll 1$, regime de super-horizonte) do raio de Hubble. De acordo com os detalhes mostrados no Apêndice C, reservando-nos a uma direção espacial específica, temos que $k \sim 1 / a_{i} \sim S / X_{i}$, logo

$$
\frac{k}{S H}=\frac{1}{X_{i} H} \sim t^{2\left(1-\sin \alpha_{i}\right) / 3}
$$

durante o período de domínio do cisalhamento. Isso mostra que, exceto para $\alpha=\pi / 2$, qualquer modo entra em um regime de super-horizonte à medida que nos aproximamos

\footnotetext{
${ }^{1}$ Por genérico queremos dizer qualquer espaço-tempo do tipo Bianchi I, exceto aquele com $\alpha=\pi / 2$. Haja visto tratar-se de um universo singular onde nenhuma das direções espaciais sofre um ricochete.

${ }^{2}$ No caso em que $\alpha=\pi / 2$, apenas um dos fatores de escala cresce enquanto os outros dois permanecem constantes. Veja a Fig. 5.1.
} 
da singularidade inicial $(t \rightarrow 0$ ), e que essa transição acontece mais "cedo" (no sentido decrescente do tempo) para os modos alinhados com a direção que sofre um ricochete. Em outras palavras, todos os modos convergem a esse regime no infinito passado. A duração desse regime, por sua vez, é mais longa para os modos alinhados com as direções oscilantes (linha azul na Fig. 7.1) do que para os modos com o mesmo valor $k_{\text {iso }}$ mas alinhados com uma direção crescente (linhas verde e vermelha na mesma figura). A existência de tais peculiaridades do modelo nos leva a duvidar da existência de um vácuo adiabático bem definido para todos os modos durante a fase de anisotropia primordial. Por outro lado, é evidente que tal estado se tornará acessível à medida que o universo se isotropiza. A questão que nos interessa, portanto, é a da validade da aproximação WKB em algum intervalo de tempo onde o cisalhamento seja pequeno mas não completamente desprezível. Tendo em mente esses pontos, passemos a uma discussão qualitativa da aproximação WKB. Inicialmente, vamos ignorar os termos de acoplamento presentes nas equações $(6.100,6.101)$ e nos focar apenas nas freqüências $\omega_{v}$ e $\omega_{\lambda}$. As soluções WKB são dadas por

$$
v_{k}^{\mathrm{WKB}}(\eta)=\frac{1}{\sqrt{2 \omega_{v}}} e^{ \pm i \int \omega_{v} \mathrm{~d} \eta}, \quad \mu_{k, \lambda}^{\mathrm{WKB}}(\eta)=\frac{1}{\sqrt{2 \omega_{\lambda}}} e^{ \pm i \int \omega_{\lambda} \mathrm{d} \eta}
$$

Como vimos, essas soluções serão soluções aproximadas das equações $(6.100,6.101)$ se $\left|Q_{v, \lambda}^{\mathrm{WKB}} / \omega_{v, \lambda}^{2}\right| \ll 1$, onde $Q_{\mathrm{WKB}}$ foi definido em (7.14). Essa quantidade não possui uma expressão analítica de fácil acesso, pois a mesma depende da solução das equações (C.5-C.7). A Fig. 7.2 ilustra a validade da aproximação WKB, obtida numericamente como função do tempo, para três modos de Fourier diferentes, cada um dos quais alinhado a um dos três eixos ortogonais das seções espaciais do universo. Podemos ver que, para um dado número de onda comóvel $k$, a condição WKB é sempre violada no passado, o que é conseqüência do regime primordial onde todos os modos estão fora do raio de Hubble. Além disso, para o modelo específico da Fig. 7.2, vemos que o modo azul é o que viola a condição WKB mais cedo (no sentido decrescente do tempo) por estar alinhado com uma direção oscilante. Podemos também verificar que, quanto maior o número de onda $k$, maior é o intervalo de duração da aproximação WKB. Isso, entretanto, não é surpresa e apenas reflete o fato de que localmente $(k \gg 1)$ o espaço-tempo é isotrópico. Para comprimentos de onda maiores que $1 / k_{\text {ref }}$ o regime WKB nunca é atingido. A Fig. 7.3 compara a solução exata (numérica) e a solução WKB para um modo $k=10 k_{\text {ref. }}$ O mesmo vale para a Fig. 7.4, mas agora levando em conta os modos tensoriais. As conclusões são essencialmente as mesmas em ambas as figuras. Todas as figuras correspondem a $\alpha=\pi / 4$. 

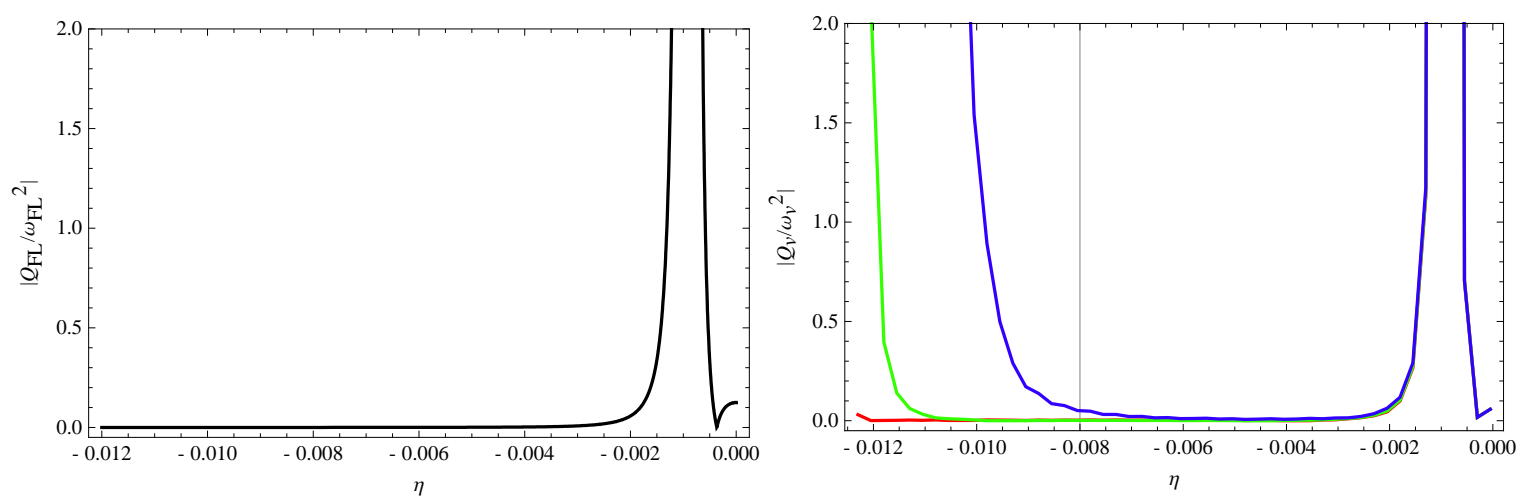

Figura 7.2: Painel esquerdo: evolução da quantidade $\left|Q^{\mathrm{WKB}} / \omega^{2}\right|$ para um universo isotrópico. Painel direito: evolução de $\left|Q_{v}^{\mathrm{WKB}} / \omega_{v}^{2}\right|$ para três diferentes modos, cada um alinhado com uma das três direções espaciais (mesma codificação de cores usada na Fig. 7.1), e com o mesmo módulo $10 k_{\text {ref }}$ no final da inflação. A linha vertical corresponde ao instante $\eta\left(\tau_{*}\right)$.
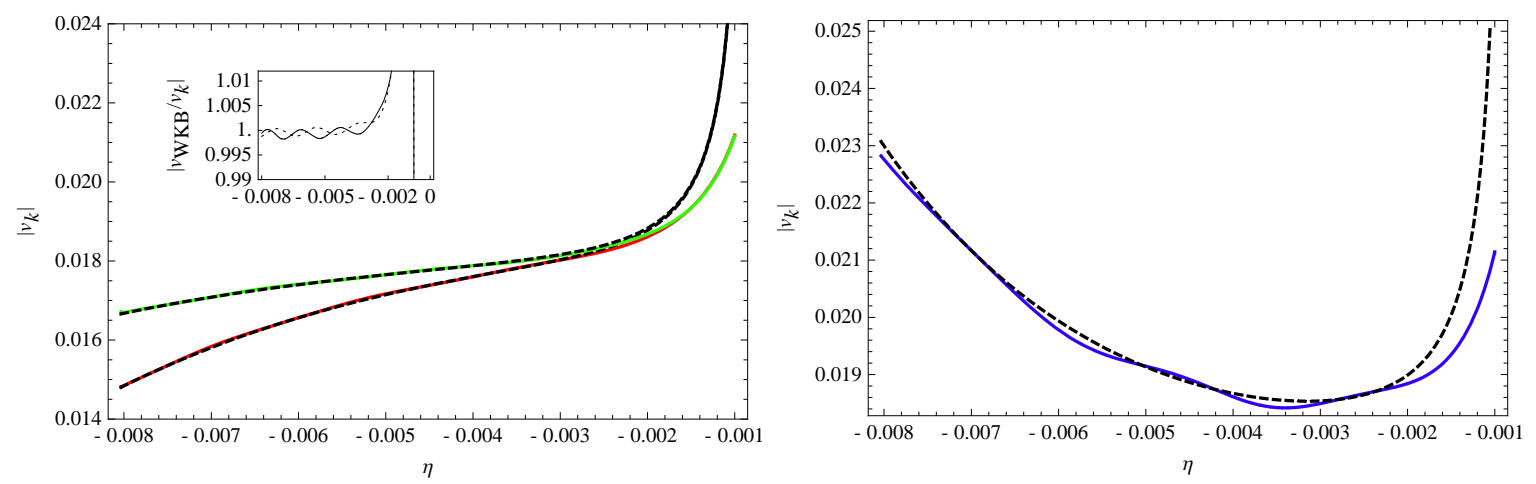

Figura 7.3: Comparação das soluções exatas (linhas sólidas) e das soluções WKB (linhas tracejadas) para o modo escalar $v_{k}$, para três vetores $\boldsymbol{k}$ ortogonais, cada qual com $k=10 k_{\text {ref }}$ no final da inflação. As figuras mostram dois modos que satisfazem a aproximação WKB (esquerda) e um modo que não a satisfaz (direita). A figura interna da esquerda mostra a razão $\left|v_{\boldsymbol{k}}^{\mathrm{WKB}} / v_{\boldsymbol{k}}\right|$ para os modos que satisfazem a aproximação WKB. 

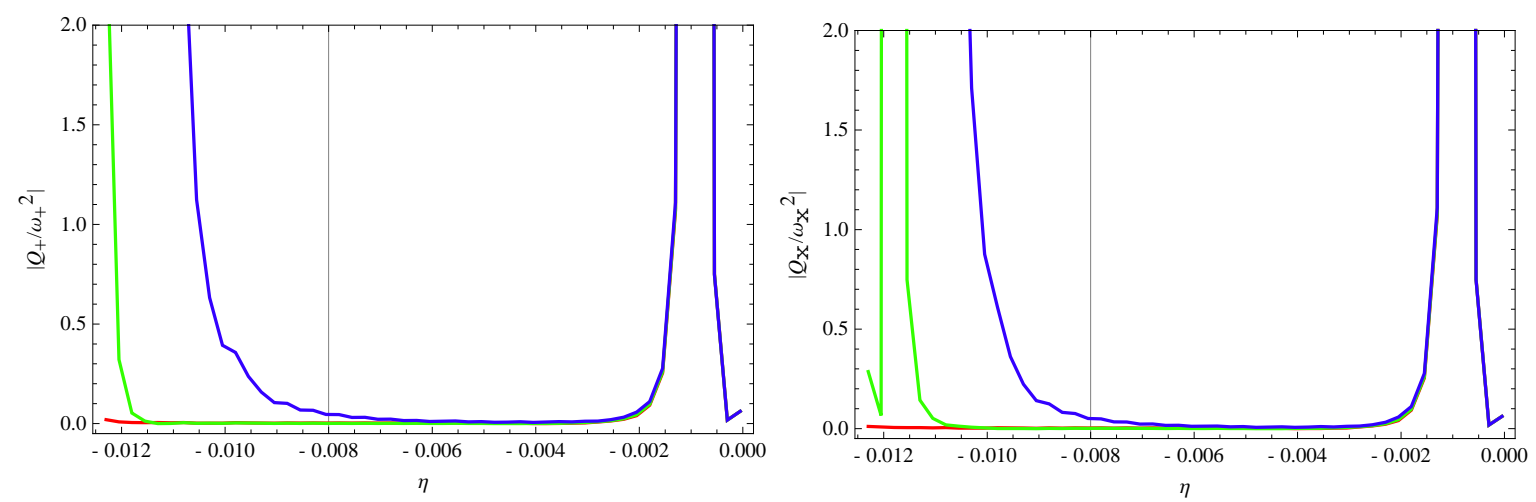

Figura 7.4: Evolução da quantidade $\left|Q_{\lambda}^{\mathrm{WKB}} / \omega_{\lambda}^{2}\right|$ para as duas polarizações tensoriais (esquerda: $\lambda=+$, direita: $\lambda=\times$ ) e para vários modos ortogonais com o mesmo

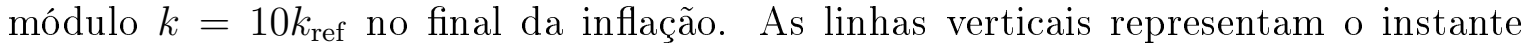
$\eta\left(\tau_{*}\right)$.

\subsubsection{Acoplamentos}

A terceira principal diferença entre a dinâmica de universos isotrópicos e anisotrópicos se deve ao acoplamento não-trivial (não-diagonalizável) entre os modos escalar e tensoriais. Conforme demonstramos em $\S 6.5$, para comprimentos de onda menores que raio de Hubble os três modos perturbativos $v, \mu_{+}$e $\mu_{\times}$se desacoplam e se comportam como uma coleção de osciladores harmônicos independentes. Entretanto, em escalas maiores os acoplamentos entre estes modos são, a priori, importantes.

A Fig. 7.5 mostra que, enquanto as funções $\aleph_{\lambda}$ que acoplam o modo escalar às ondas gravitacionais podem ser negligenciadas em pequenas escalas, esse certamente não é o caso da função $\beth$ que acopla as diferentes polarizações das ondas gravitacionais. Tal função não pode ser ignorada, mesmo nos instantes iniciais, para modos que não sejam grandes o suficiente. Tipicamente os modos a partir dos quais esse acoplamento não pode ser negligenciado são os mesmos que violam a aproximação de WKB, ou seja,

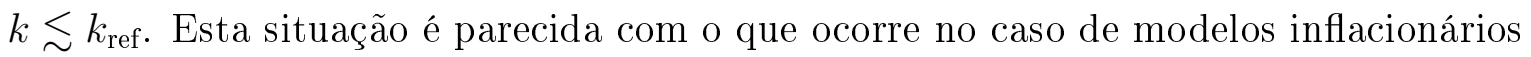
com vários campos dinâmicos. Nesses modelos os campos também estão, em geral, acoplados (veja [96] para uma referência recente). Entretanto, mesmo nesses casos, é possível extrair campos independentes no regime de sub-horizonte [97], de modo que a introdução de um conjunto de variáveis estocásticas independentes é ainda possível.

Em resumo, para sistemas onde o acoplamento dos modos não é trivial, uma descrição fiel da dinâmica das perturbações em grandes comprimentos de onda é de difícil acesso, uma vez que tais perturbações não podem ser consideradas independentes. 

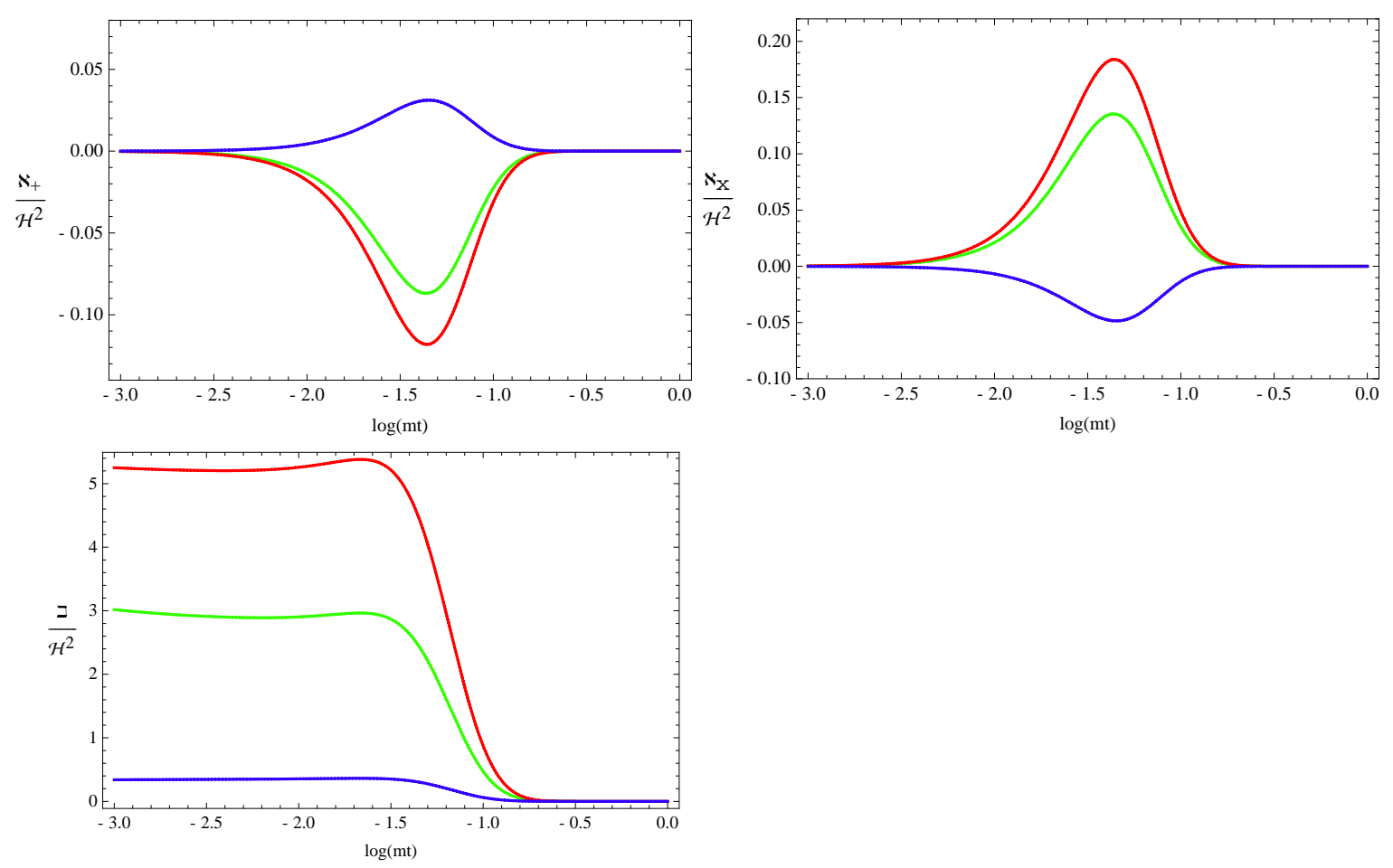

Figura 7.5: Evolução das funções $\aleph_{\lambda} / \mathcal{H}^{2}$ (esquerda: $\lambda=+$, direita: $\lambda=\times$ ) e $\beth / \mathcal{H}^{2}$ para três modos alinhados a três eixos ortogonais arbitrários (representados por cores diferentes).

\subsection{Prescrição para as condições iniciais}

Tendo em mente a discussão da seção anterior, vamos adotar a seguinte prescrição para definir nossas condições iniciais: para um dado modo $k$, as condições iniciais serão definidas no instante em que a quantidade $\left|Q^{\mathrm{WKB}} / \omega^{2}\right|$ é mínima. Esse instante, digamos $t=t_{i}(\boldsymbol{k})$, depende explicitamente do modo $\boldsymbol{k}$. Isso, no entanto, não representa um problema já que todos os modos são independentes uns dos outros.

Como vimos, para os modos tais que $k \lesssim k_{\text {ref }}$ o regime WKB nunca é atingido. Além disso, visto que os modos com $k_{0}=S_{0} H_{0}$ foram, por definição, excitados durante a inflação e têm, ao menos aproximadamente, um espectro de potências invariante de escala, devemos assumir que $k_{\text {ref }} \lesssim k_{0}$. Segue portanto das Figs. 7.6 e 7.7 que o regime WKB é alcançado por todos os modos com $k>k_{\text {ref. }}$ Na verdade, isso corresponde a um ajuste fino do valor da anisotropia inicial do universo de modo que apenas os maiores modos observáveis hoje tenham sido afetados pela fase de anisotropia primordial.

Para checar a consistência desse procedimento, nós variamos o instante $t_{i}(\boldsymbol{k})$. Em particular, também verificamos o efeito do instante $t_{i}(\boldsymbol{k})=\tau_{*}$ para todos os modos. O resultado dessa análise mostrou-se pouco sensível à escolha de $t_{i}(\boldsymbol{k})$ para modos $k \gtrsim 2 k_{\text {ref }}$ e altamente dependente do mesmo para grandes comprimentos de onda. Isso advém do fato de que a duração do regime WKB para modos menores que $2 k_{\text {ref é tanto }}$ menor quanto maior for o comprimento de onda considerado. Notemos também que este procedimento é mais robusto para os modos alinhados com as direções estritamente crescentes do universo. 
A Fig. 7.8 mostra que os termos de acoplamento nas Eqs. $(6.100,6.101)$ podem ser desprezados, com boa aproximação, para os modos $k \gtrsim 2 k_{\text {ref. É importante enfatizar }}$ também que essa hipótese se torna inválida aproximadamente no mesmo instante em que a aproximação WKB deixa de valer. Portanto, uma vez tomadas as devidas precauções, assumiremos que os três modos perturbativos $v, \mu_{+}$e $\mu_{\times}$são independentes no exato momento em que são excitados pelas flutuações quânticas. Em outras palavras, a anisotropia que seremos capazes de quantificar advém da dependência direcional das freqüências $\omega_{v}$ e $\omega_{\lambda}$, e não dos termos de acoplamento $\aleph_{\lambda}$ e $\beth$.

Tecnicamente, a implementação numérica deste procedimento parte das seguintes soluções

$$
v_{\boldsymbol{k}}=\frac{e^{-i \int \omega_{v} \mathrm{~d} \eta}}{\sqrt{2 \omega_{v}(\boldsymbol{k}, \tau)}} e_{v}(\boldsymbol{k}), \quad \text { e } \quad \mu_{\boldsymbol{k}, \lambda}=\frac{e^{-i \int \omega_{\lambda} \mathrm{d} \eta}}{\sqrt{2 \omega_{\lambda}(\boldsymbol{k}, \tau)}} e_{\lambda}(\boldsymbol{k})
$$

definidas a menos de fases arbitrárias que podem ser absorvidas na definição das variáveis aleatórias. Estas, por sua vez, satisfazem

$$
\left\langle e_{X}(\boldsymbol{k}) e_{Y}^{*}(\boldsymbol{q})\right\rangle=\delta_{X Y} \delta^{(3)}(\boldsymbol{k}-\boldsymbol{q}) .
$$

Tendo as equações acima como condições iniciais, o sistema de Eqs. $(6.100,6.101)$ pode então ser solucionado a partir do instante $t_{i}(\boldsymbol{k})$ até o fim da inflação (aproximadamente 60 e-folds).
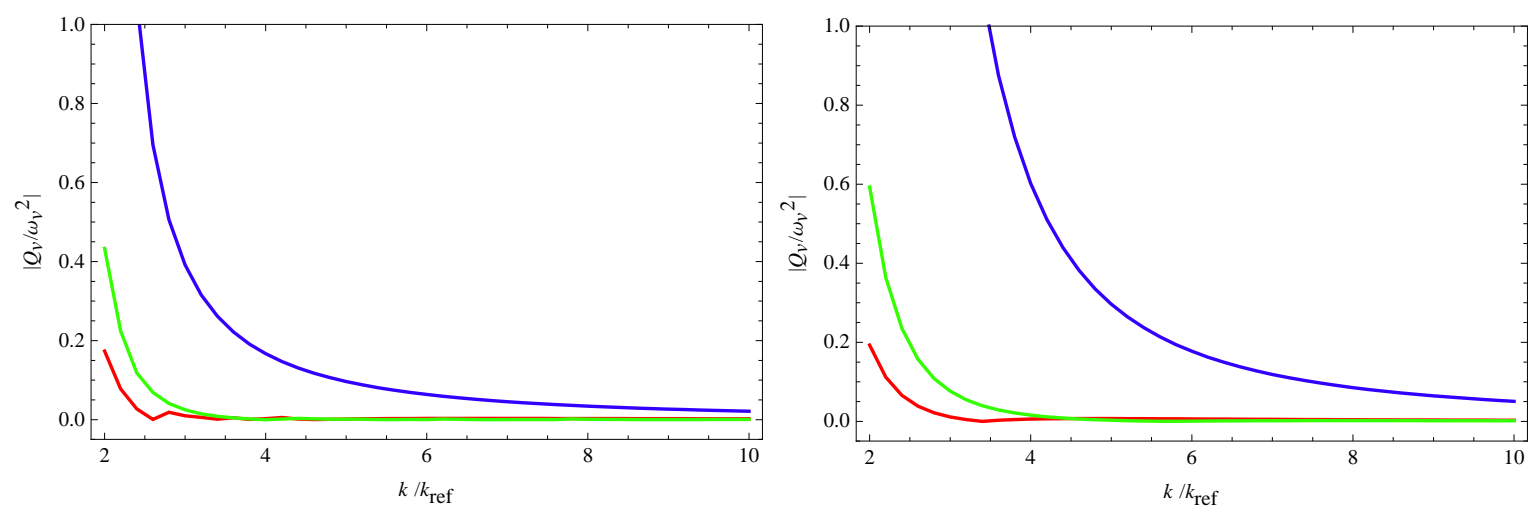

Figura 7.6: Validade da aproximação WKB para a perturbação $v_{\boldsymbol{k}}$ em função do módulo de $\boldsymbol{k}$, calculada no instante em que as condições iniciais são implementadas (em $t=t_{i}(\boldsymbol{k})$ à esquerda e $t=\tau_{*}$ à direita). As cores correspondem às três direções espaciais ortogonais e o modelo considerado equivale a $\alpha=\pi / 4$. 

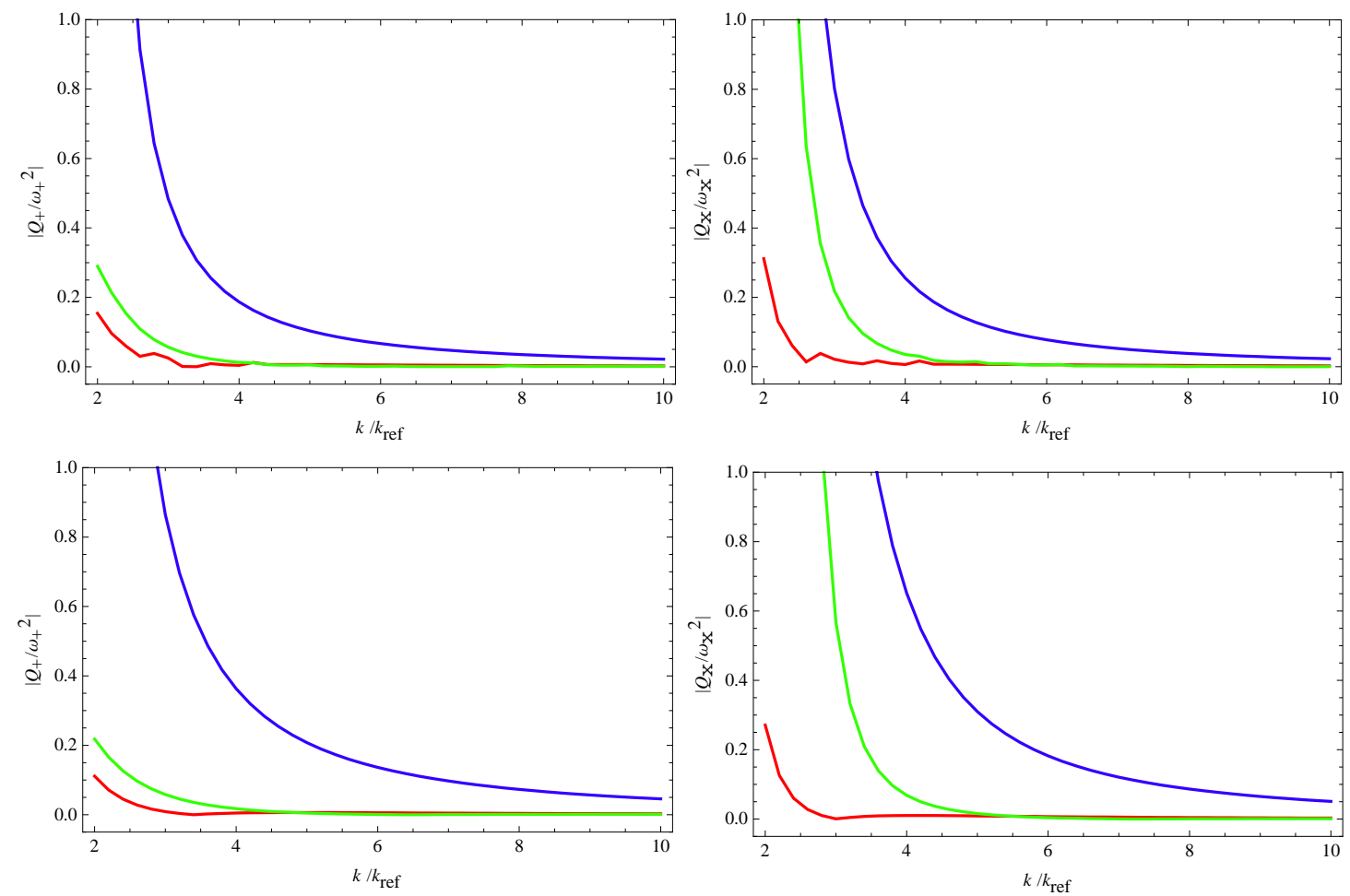

Figura 7.7: Aproximação WKB para os perturbações tensoriais. A figura mostra a quantidade $\left|Q_{\lambda}^{\mathrm{WKB}} / \omega_{\lambda}^{2}\right|$ para $\lambda=+$ (painel esquerdo) e $\lambda=\times$ (painel direito) para modos alinhados às três direções ortogonais. As condições iniciais foram definidas em $t=t_{i}(\boldsymbol{k})$ (painéis superiores) e em $t=\tau_{*}$ (painéis inferiores).
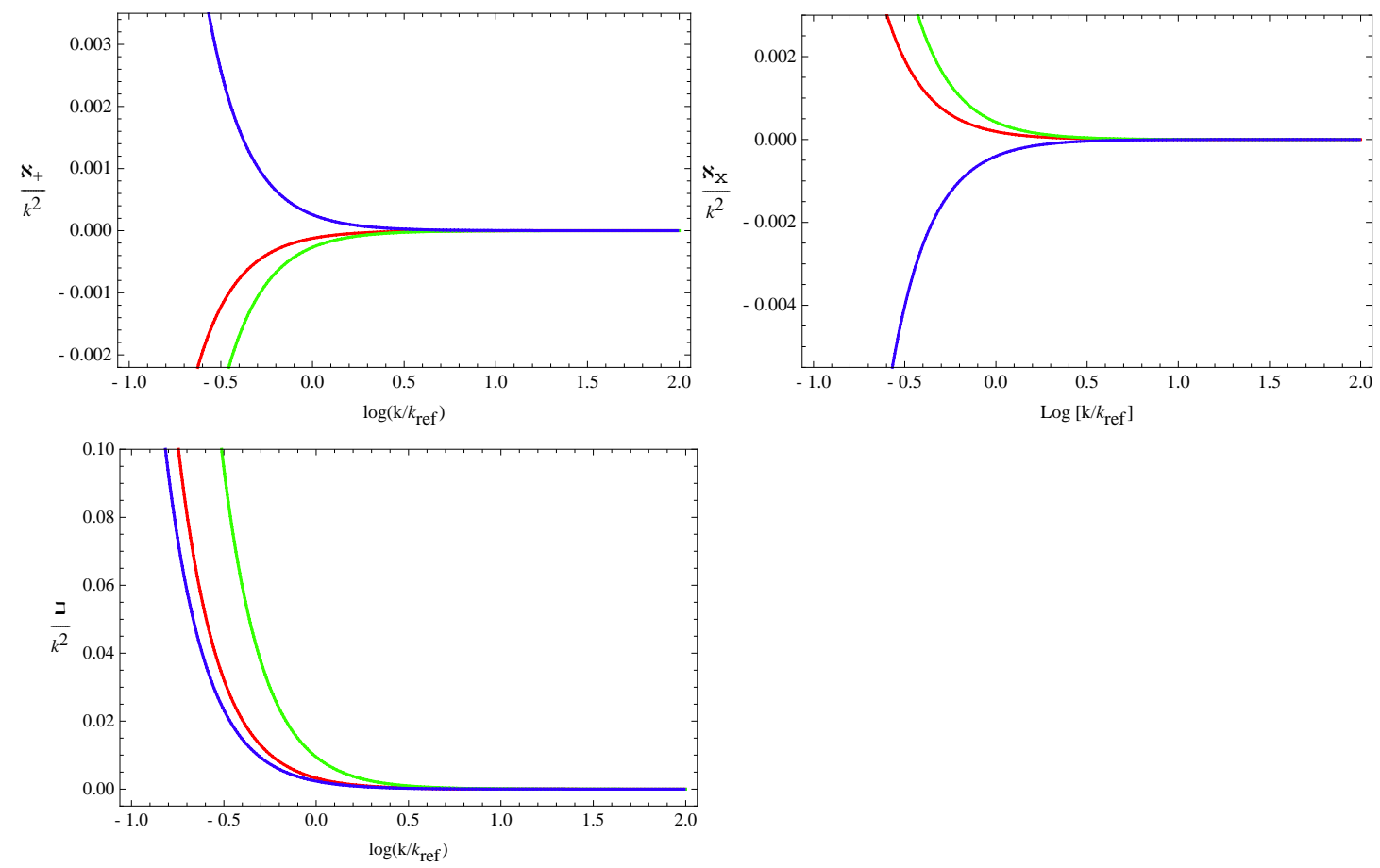

Figura 7.8: Independência das perturbações em $t=\tau_{*}$. A figura mostra $\aleph_{+} / k^{2}$ (esquerda), $\aleph_{\times} / k^{2}$ (meio) e $\beth / k^{2}$ em função de $k$. 


\subsection{Liberdade da parametrização temporal}

Durante a elaboração deste trabalho, uma equipe de pesquisadores argumentou [23] que o sistema dinâmico de equações para as variáveis de Mukhanov-Sasaki admite reparametrizações temporais que conservam o caráter canônico do mesmo, no sentido de que para uma dada variável canônica $v(\eta)$ que satisfaz

$$
\frac{\mathrm{d}^{2} v}{\mathrm{~d} \eta^{2}}+\omega_{v}^{2} v=0 \quad \text { e } \quad v \frac{\mathrm{d} v^{*}}{\mathrm{~d} \eta}-v^{*} \frac{\mathrm{d} v}{\mathrm{~d} \eta}=i,
$$

existe uma função $f$ e uma variável temporal $\tau$, definidas como

$$
f(\eta)^{2} \mathrm{~d} \tau=\mathrm{d} \eta
$$

através das quais podemos definir uma nova variável, $u=f v$, que satisfaz

$$
\frac{\mathrm{d}^{2} u}{\mathrm{~d} \tau^{2}}+\omega_{u}^{2} u=0 \quad \text { and } \quad u \frac{\mathrm{d} u^{*}}{\mathrm{~d} \tau}-u^{*} \frac{\mathrm{d} u}{\mathrm{~d} \tau}=i
$$

Se, por exemplo, $v$ é uma variável para a qual a condição WKB não vale, podemos nos perguntar se existe uma função $f$ que nos leve a uma conclusão diferente no que diz respeito à validade da aproximação WKB para a variável $u$. No nosso caso, a reparametrização temporal definida na Eq. (7.35) leva o sistema de Eqs. (6.100, 6.101) a um sistema idêntico de equações, porém satisfeito pelas novas variáveis

$$
\tilde{v} \equiv f v, \quad \tilde{\mu}_{+} \equiv f \mu_{+}, \quad \tilde{\mu}_{\times} \equiv f \mu_{\times}
$$

sujeitas agora às novas freqüências

$$
\tilde{\omega}_{v}^{2} \equiv \frac{\omega_{v}^{2}}{f^{4}}-\frac{1}{f} \frac{\mathrm{d}^{2} f}{\mathrm{~d} \tau^{2}}, \quad \tilde{\omega}_{\lambda}^{2} \equiv \frac{\omega_{\lambda}^{2}}{f^{4}}-\frac{1}{f} \frac{\mathrm{d}^{2} f}{\mathrm{~d} \tau^{2}},
$$

e às novas funções de acoplamento

$$
\tilde{\aleph}_{\lambda} \equiv \frac{\aleph_{\lambda}}{f^{4}}, \quad \tilde{\beth} \equiv \frac{\beth}{f^{4}}
$$

Por simplicidade, omitiremos as funções de acoplamento nas discussões seguintes. Como já mencionado, no trabalho [23] demonstrou-se que, se $\omega_{v}$ e $\omega_{\lambda}$ são freqüências que satisfazem a condição WKB, então para que as novas freqüências também satisfaçam a aproximação, a função $f$ não pode ser arbitrária mas deve satisfazer as condições

$$
\frac{1}{f}\left|\frac{\mathrm{d}^{n} f}{\mathrm{~d} \eta^{n}}\right| \ll \omega_{v}^{n}, \omega_{\lambda}^{n}, \quad \text { com } n \in\{1,2,3,4\} .
$$

Sob tais circunstâncias, a quantização do conjunto de variáveis $\left(v, \mu_{+}, \mu_{\times}\right)$e $\left(\tilde{v}, \tilde{\mu}_{+}, \tilde{\mu}_{\times}\right)$ leva aos mesmos observáveis. É importante enfatizar que a condição para $n=4$ é necessária para que a aproximação correta $\left|Q^{\mathrm{WKB}} / \omega^{2}\right| \ll 1$ seja obtida, ao invés da condição $\omega^{\prime} / \omega^{2} \ll 1$ considerada em [23].

No nosso caso, as freqüências $\omega_{v}$ e $\omega_{\lambda}$ se comportam como $S H \sim 1 / \eta$ quando $t \rightarrow 0$. Seja $W_{0}$ a constante de integração na Eq. (5.42) tal que a singularidade inicial 
corresponda ao instante $\eta=0$. Então, se $\omega_{v, \lambda}=C / \eta$, as funções $Q_{v, \lambda}^{\text {WKB }}$ se comportarão como

$$
\frac{Q_{v, \lambda}^{\mathrm{WKB}}}{\omega_{v, \lambda}^{2}}=-\frac{1}{4 C^{2}} .
$$

Consideremos agora, por simplicidade, uma reparametrização temporal definida pela função $f(\eta)=\eta^{A}$. Em termos da nova variável temporal, a condição WKB fica

$$
\frac{\tilde{Q}_{\tilde{v}, \tilde{\lambda}}^{\mathrm{WKB}}}{\tilde{\omega}_{\tilde{v}, \tilde{\lambda}}^{2}}=-\frac{\left(A+\frac{1}{2}\right)^{2}}{C^{2}+A(A+1)} .
$$

Esse resultado mostra que, para a reparametrização particular definida por $A=-1 / 2$, é possível obter um regime WKB para as perturbações $\left(\tilde{v}, \tilde{\mu}_{+}, \tilde{\mu}_{\times}\right)$, desde que tenhamos $|C| \neq 1 / 2$. Entretanto, usando a expansão da equação (C.28) e os comportamentos assintóticos obtidos nas equações (5.61), concluímos que quando o termo $z_{s}^{\prime \prime} / z_{s}$ é dominante frente à frequência $\omega_{v}^{2}$, temos que $\omega_{v}^{2} \rightarrow-\mathcal{H}^{2}$. Isso implica que, para esta frequência, a constante $|C|$ vale precisamente $1 / 2$ e portanto $f(\eta)=\eta^{-1 / 2}$ não pode ser considerada uma reparametrização física do tempo.

Concluímos portanto que não é possível, pelo menos para a classe de reparametrizações consideradas, construir uma redefinição do tempo que mude o comportamento WKB de uma variável canônica qualquer. A escolha de variáveis canônicas é única a menos de reparametrizações que satisfazem as condições (7.40). A única exceção ocorre quando o termo dominante em $\omega_{v}^{2}$ é $k^{2}$, como acontece no modelo particular $\alpha=\pi / 2$. Nesse caso, $k \sim t^{-2 / 3} \sim \eta^{-1}$ e as conclusões obtidas em [23] são recuperadas. Isso mostra novamente o quão singular é o universo definido por $\alpha=\pi / 2$.

\subsection{Discussão}

Vamos discutir agora o procedimento usado para definir as condições iniciais. Em primeiro lugar, nossa análise revelou que os modos menores que $k_{\text {ref nunca atingem }}$ um regime WKB. Para esses modos nós não possuímos uma prescrição consistente de quantização capaz de determinar suas amplitudes. Uma saída para esse problema, que não foi analisada por nós, seria fixar a amplitude desses modos assumindo que as mesmas minimizam a energia de cada modo, assim como proposto em [98] no contexto de modelos trans-planckianos que violam o regime WKB.

Uma outra solução, talvez mais conservadora, seria assumir que inicialmente esses modos assumem valores completamente aleatórios, introduzindo para isso uma função livre. Tal função poderia então ser vinculada, por exemplo, através das flutuações em grandes escalas da radiação cósmica de fundo, ou mesmo como parte de algum processo ainda desconhecido mas presente em uma escala fundamental, como a escala de Planck. Portanto, na ausência de uma receita bem definida para determinar a amplitude desses modos nós perdemos qualquer poder de previsão da física das grandes escalas. Entretanto, tais escalas podem ainda estar além do raio observável do universo. Nesse sentido nossos resultados nos levam de volta aos momentos (históricos) da pré-inflação, onde a reprodução das observações em grandes escalas dependia da introdução $a d-h o c$ de um espectro de potências do tipo Harrison-Zel'dovich. É aqui que se reconhece um dos maiores trunfos do modelo inflacionário: o de predizer, a 
partir de primeiros princípios, a forma desse espectro. Nossa análise mostra portanto que as previsões do modelo inflacionário padrão são frágeis frente à existência de uma fase clássica de anisotropia primordial. Previsões robustas resultarão provavelmente de teorias apropriadas $[99,100,101]$ a essas escalas, ou de teorias que permitam um mecanismo de criação dessa fase anisotrópica [102].

Do ponto de vista prático, podemos estimar o efeito das funções de acoplamento $\aleph_{\lambda}$ e $\beth$ mudando arbitrariamente suas amplitudes. Pudemos verificar que o impacto dessa mudança sobre as previsões apresentadas na seção seguinte são muito pequenas. Em particular, isso nos mostra que a dependência direcional dos espectros de potência decorrem essencialmente da dependência direcional dos números de onda comóveis (veja Fig. 7.1). Também verificamos que à medida que a razão $k / k_{\text {ref }}$ aumenta, nossas previsões convergem às obtidas no caso do modelo inflacionário padrão. Concluímos portanto que nossa prescrição é confiável apenas para modos maiores que $k_{\text {ref }}$ e que a mesma depende da hipótese de que $k_{\text {ref }}$ seja menor que $k_{0}$. Esse último detalhe pode ser visto como uma "hipótese observacional", mas também como um ajuste fino na quantidade de cisalhamento primordial que não pode ser explicada pelo presente modelo. Nesse regime nossas previsões são robustas e permanecem praticamente inalteradas pelas perturbações arbitrárias e pré-existentes ao período inflacionário e que fixam as propriedades dos modos de grandes comprimentos de onda.

\subsection{Espectros das Perturbações Primordiais}

Uma das motivações iniciais desse trabalho é entender o impacto das hipóteses de simetria do universo primordial sobre a radiação cósmica de fundo. Essa análise depende da forma específica dos espectros primordiais das perturbações que, em contrapartida, dependem das soluções exatas do sistema de equações (6.100-6.101). Não obstante as especificidades do modelo, os espectros finais devem ser compatíveis com as simetrias gerais do espaço-tempo de fundo. Resulta dessa necessidade física que a função de correlação de temperatura da RCF deve satisfazer certas "regras de seleção", as quais podem eventualmente conter informações sobre a origem das anomalias de pequenos multipolos mencionadas na introdução deste trabalho. Portanto, antes de passarmos à implementação numérica das equações e à conseqüente análise dos resultados, é importante conhecermos a forma geral da função de correlação de temperatura compatível com um universo anisotrópico.

\subsubsection{Características gerais}

Consideremos um campo gaussiano e real de temperatura, $\Delta T(\boldsymbol{n})$, definido sobre a esfera celeste. Devido à simetria do problema, é conveniente decompor essa função numa base harmônica de funções esféricas através dos coeficientes

$$
a_{\ell m}=\int \mathrm{d} \Omega_{\hat{\boldsymbol{n}}} \Delta T(\boldsymbol{n}) Y_{\ell m}(\hat{\boldsymbol{n}}) .
$$

A função de correlação de temperatura é definida como uma média, sobre várias realizações, do produto da flutuação de temperatura $\Delta T$, calculada em dois pontos arbitrários da esfera celeste, $\hat{\boldsymbol{n}}_{1}$ e $\hat{\boldsymbol{n}}_{2}$. Equivalentemente, a função de correlação pode ser escrita em termos dos coeficientes harmônicos $a_{\ell m}$. Para tanto trabalharemos no 
espaço de Fourier e tomaremos a decomposição das ondas planas $e^{\mathbf{i} \boldsymbol{k} \cdot \boldsymbol{n}}$ em termos das funções harmônicas $Y_{\ell m}$ (Apêndice A). Assim sendo a função de correlação fica

$$
\left\langle a_{\ell_{1} m_{1}} a_{\ell_{2} m_{2}}^{*}\right\rangle=\mathrm{i}^{\ell_{1}}(-\mathrm{i})^{\ell_{2}}(4 \pi)^{2} \int \frac{\mathrm{d}^{3} k \mathrm{~d}^{3} q}{(2 \pi)^{3}}\langle\Delta T(\boldsymbol{k}) \Delta T(\boldsymbol{q})\rangle j_{\ell_{1}}(k R) j_{\ell_{2}}(q R) Y_{\ell_{1} m_{1}}^{*}(\hat{\boldsymbol{k}}) Y_{\ell_{2} m_{2}}(\hat{\boldsymbol{q}}),
$$

sendo $R$ o raio da esfera de último espalhamento entre os fótons e a matéria do plasma primordial (veja a discussão feita em $\S 1.2$ ). A função $\langle\Delta T(\boldsymbol{k}) \Delta T(\boldsymbol{q})\rangle$ é a correlação de temperatura no espaço de Fourier. Se o espaço-tempo de fundo for homogêneo, a função de correlação deve ter simetria translacional, portanto deve ser da forma

$$
\langle\Delta T(\boldsymbol{k}) \Delta T(\boldsymbol{q})\rangle=2 \pi^{2} k^{-3} \mathcal{P}(\boldsymbol{k}) \delta^{(3)}(\boldsymbol{k}-\boldsymbol{q})
$$

onde consideramos apenas a contribuição das perturbações de grandes comprimentos de onda $(k / a H \rightarrow \infty)$ para as flutuações de temperatura e $\mathcal{P}(\boldsymbol{k})$ é o espectro de potência das perturbações, que podem ser escalares ou tensoriais. Se além de homogêneo, supusermos que o espaço é também isotrópico, então o espectro deve depender apenas do módulo do vetor $\boldsymbol{k}: \mathcal{P}=\mathcal{P}(k)$. Quando esse for o caso, a parte angular da integral (7.44) pode ser calculada, resultando em

$$
\left\langle a_{\ell_{1} m_{1}} a_{\ell_{2} m_{2}}^{*}\right\rangle=C_{\ell_{1}} \delta_{\ell_{1} \ell_{2}} \delta_{m_{1} m_{2}}, \quad C_{\ell_{1}}=4 \pi \int_{0}^{\infty} \frac{\mathrm{d} k}{k} \mathcal{P}(k) j_{\ell_{1}}^{2}(k R)
$$

A presença das deltas de Kronecker na matriz de covariância $\left\langle a_{\ell_{1} m_{1}} a_{\ell_{2} m_{2}}^{*}\right\rangle$ assegura a invariância da função de correlação por rotações. Ou seja, se o universo for homogêneo e isotrópico, os coeficientes $C_{\ell_{1}}$ especificam completamente a função de correlação da RCF. Se quisermos abrir mão da hipótese de isotropia, então o espectro de potência (7.45) deve depender também da direção dos vetores $\boldsymbol{k}: \mathcal{P}=\mathcal{P}(\boldsymbol{k})$. Nesse caso sua dependência angular pode ser acomodada em outra decomposição harmônica

$$
\mathcal{P}(\boldsymbol{k})=\sum_{\ell_{3}, m_{3}} r_{\ell_{3} m_{3}}(k) Y_{\ell_{3} m_{3}}(\hat{\boldsymbol{k}}) .
$$

Assim sendo a matriz de covariância se reescreve na forma [17]

$$
\left\langle a_{\ell_{1} m_{1}} a_{\ell_{2} m_{2}}^{*}\right\rangle=\sum_{\ell_{3}, m_{3}} \mathcal{G}_{m_{1} m_{2} m_{3}}^{\ell_{1} \ell_{2} \ell_{3}} D_{\ell_{1} \ell_{2}}^{\ell_{3} m_{3}}, \quad \ell_{3} \in 2 \mathbb{Z}^{+}
$$

na qual

$$
\begin{aligned}
\mathcal{G}_{m_{1} m_{2} m_{3}}^{\ell_{1} \ell_{2} \ell_{3}} & =(-1)^{m_{1}} \int \mathrm{d} \Omega_{\hat{\boldsymbol{k}}} Y_{\ell_{3} m_{3}}(\hat{\boldsymbol{k}}) Y_{\ell_{1},-m_{1}}(\hat{\boldsymbol{k}}) Y_{\ell_{2} m_{2}}(\hat{\boldsymbol{k}}), \\
D_{\ell_{1} \ell_{2}}^{\ell_{3} m_{3}} & =4 \pi i^{\ell_{1}-\ell_{2}} \int_{0}^{\infty} \frac{\mathrm{d} k}{k} r_{\ell_{3} m_{3}}(k) j_{\ell_{1}}(k R) j_{\ell_{2}}(k R) .
\end{aligned}
$$

Os momentos $D_{\ell_{1} \ell_{2}}^{\ell_{3} m_{3}}$ representam a generalização anisotrópica dos coeficientes isotrópicos $C_{\ell_{1}}$ para $\ell_{3}>0$. Alguns comentários sobre as características da decomposição acima são necessários. Em primeiro lugar, notemos que a paridade do momento multipolar $\ell_{3}$ decorre da invariância do espectro (7.47) por transformações de paridade, 
ou seja, $\mathcal{P}(\boldsymbol{k})=\mathcal{P}(-\boldsymbol{k})$. Essa invariância é uma conseqüência direta da homogeneidade do espaço-tempo de fundo. Em segundo lugar, os coeficientes $\mathcal{G}_{m_{1} m_{2} \ell_{2} \ell_{2} \ell_{3}}^{\ell_{3}}$ representam as características puramente geométricas de um sistema cujo momento angular total $\left(\ell_{3}\right)$ é dado pela combinação de dois componentes independentes de momento angular $\left(\ell_{1}, \ell_{2}\right)$. Esses coeficientes naturalmente selecionam combinações admissíveis dos momentos angulares e são identicamente nulos para quaisquer valores de $\left(\ell_{i}, m_{i}\right)$, $i \in(1,2,3)$, que não satisfaçam as seguintes condições

$$
\begin{cases}\ell_{1}+\ell_{2}+\ell_{3} & \in 2 \mathbb{Z}^{+} \\ m_{1}+m_{2}+m_{3} & =0 \\ \left|\ell_{i}-\ell_{j}\right| \leq \ell_{k} \leq \ell_{i}+\ell_{j} & \forall i, j, k \in\{1,2,3\}\end{cases}
$$

Aplicadas à função de correlação (7.48), essas regras de seleção implicam que

$$
\left\langle a_{\ell_{1} m_{1}} a_{\ell_{2} m_{2}}^{*}\right\rangle=0, \quad \forall\left\{\ell_{1}, \ell_{2} ;\left|\ell_{1}-\ell_{2}\right| \in 2 \mathbb{Z}^{+}+1\right\}
$$

e, em particular, $\left\langle a_{2 m_{1}} a_{3 m_{2}}\right\rangle=0$. Isso mostra que correlações entre os coeficientes de quadrupolo e de octopolo não podem surgir numa realização aleatória de um universo homogêneo e anisotrópico. É importante enfatizar que essa é uma conseqüência puramente geométrica de um universo anisotrópico e independente de modelos específicos de inflação. De fato, é o que se observa explicitamente nos modelos introduzidos em $[2,23,24]$. Esse resultado sugere que o alinhamento entre o dipolo e o quadrupolo da RCF seja, talvez, uma manifestação da quebra de homogeneidade do universo.

A paridade do espectro anisotrópico também impõe regras de seleção sobre os coeficientes da decomposição (7.47), pois sendo homogêneo, o universo de Bianchi deve ser também invariante por reflexões sobre os planos $x y, x z$ e $y z$. Escrevendo as funções harmônicas esféricas na forma

$$
\left.Y_{\ell_{3} m_{3}}(x, y, z) \propto\left(\frac{\partial}{\partial z}\right)^{\ell_{3}-m_{3}}\left(\frac{\partial}{\partial x}+i \frac{\partial}{\partial y}\right)^{m_{3}} \frac{1}{r}\right|_{r=1},
$$

impondo a invariância do espectro sob a transformação $z \rightarrow-z$ e, em seguida, usando a paridade de $\ell_{3}$, concluiremos que isso só é possível se $(-1)^{\ell_{3}-m_{3}}=1$, ou seja, $m_{3}$ deve também ser um número par. Repetindo o procedimento para as transformações $x \rightarrow-x$ e $y \rightarrow-y$, encontramos respectivamente as condições $r_{\ell_{3} m_{3}}=r_{\ell_{3},-m_{3}} \mathrm{e}$ $r_{\ell_{3} m_{3}}^{*}=r_{\ell_{3},-m_{3}}$. Portanto, os únicos componentes não-nulos em (7.47) são da forma

$$
r_{\ell_{3} m_{3}} \in \mathbb{R}, \quad\left(\ell_{3}, m_{3}\right) \in 2 \mathbb{Z}^{+}
$$

Ou seja, dos $2 \ell_{3}+1$ coeficientes $r_{\ell_{3} m_{3}}$ em (7.47), apenas $1+\ell_{3} / 2$ são compatíveis com as simetrias do universo de Bianchi I.

\subsubsection{Implementação numérica}

Antes que possamos implementar as condições iniciais como previamente detalhado precisamos solucionar (numericamente) o sistema de equações (6.100, 6.101). Devido aos termos de acoplamento, cada uma das três variáveis $v, \mu_{+}$e $\mu_{\times}$terá componentes 
ao longo das três "direções" estocásticas, mesmo que as mesmas sejam inicialmente independentes. Por exemplo,

$$
v_{\boldsymbol{k}}(\eta)=v_{v}(\boldsymbol{k}, \eta) e_{v}(\boldsymbol{k})+v_{+}(\boldsymbol{k}, \eta) e_{+}(\boldsymbol{k})+v_{\times}(\boldsymbol{k}, \eta) e_{\times}(\boldsymbol{k})
$$

e

$$
\mu_{\boldsymbol{k}, \lambda}(\eta)=\mu_{\lambda v}(\boldsymbol{k}, \eta) e_{v}(\boldsymbol{k})+\mu_{\lambda \lambda}(\boldsymbol{k}, \eta) e_{\lambda}(\boldsymbol{k})+\mu_{\lambda(1-\lambda)}(\boldsymbol{k}, \eta) e_{1-\lambda}(\boldsymbol{k}) .
$$

A partir das propriedades das variáveis aleatórias, podemos deduzir que

$$
\left\langle v_{\boldsymbol{k}}(\eta) v_{\boldsymbol{k}^{\prime}}^{*}(\eta)\right\rangle=\left(\left|v_{v}(\boldsymbol{k}, \eta)\right|^{2}+\left|v_{+}(\boldsymbol{k}, \eta)\right|^{2}+\left|v_{\times}(\boldsymbol{k}, \eta)\right|^{2}\right) \delta^{(3)}\left(\boldsymbol{k}-\boldsymbol{k}^{\prime}\right)
$$

o mesmo sendo válido para $\mu_{\lambda}$. Ao fim da inflação a anisotropia primordial se torna completamente negligenciável e o espectro de potências associado à perturbação de curvatura fica determinado por

$$
P_{\mathcal{R}}(\boldsymbol{k})=\frac{2 \pi^{2}}{k^{3}} \mathcal{P}_{\mathcal{R}}(\boldsymbol{k})=\frac{1}{z_{\mathrm{S}}^{2}}\left(\left|v_{v}(\boldsymbol{k}, \eta)\right|^{2}+\left|v_{+}(\boldsymbol{k}, \eta)\right|^{2}+\left|v_{\times}(\boldsymbol{k}, \eta)\right|^{2}\right)
$$

Podemos verificar que, para modos maiores que o raio de Hubble, a quantidade $\mathcal{R}$ se conserva logo que o cisalhamento se torna negligenciável, como esperado. Dessa forma nossa análise numérica deve ser feita em um intervalo de tempo grande o suficiente de modo a garantir que o universo tenha se isotropizado e que todos os modos tenham se tornado maiores que o raio de Hubble. De maneira análoga, os espectros de potências das ondas gravitacionais são definidos por

$$
P_{\lambda}(\boldsymbol{k})=\frac{2 \pi^{2}}{k^{3}} \mathcal{P}_{\lambda}(\boldsymbol{k})=\left|\mu_{\lambda v}(\boldsymbol{k}, \eta)\right|^{2}+\left|\mu_{\lambda \lambda}(\boldsymbol{k}, \eta)\right|^{2}+\left|\mu_{\lambda(1-\lambda)}(\boldsymbol{k}, \eta)\right|^{2}
$$

Após o fim da inflação e começo da era de domínio da radiação, o espaço-tempo de fundo pode ser descrito pela métrica de FRW. Nesse caso, a física da fase primordial de anisotropia estará codificada nas propriedades estatísticas das perturbações de grandes comprimentos de onda. Para termos acesso a elas é conveniente decompor os espectros de potências de acordo com (7.47), ou seja

$$
\mathcal{P}_{\mathcal{R}}(\boldsymbol{k})=f_{\mathcal{R}}(k)\left[1+\sum_{\ell=1}^{\ell=\infty} \sum_{m=-\ell}^{m=+\ell} r_{\ell m}(k) Y_{\ell m}(\hat{\boldsymbol{k}})\right]
$$

e

$$
\mathcal{P}_{\lambda}(\boldsymbol{k})=f_{\lambda}(k)\left[1+\sum_{\ell=1}^{\ell=\infty} \sum_{m=-\ell}^{m=+\ell} r_{\ell m}^{\lambda}(k) Y_{\ell m}(\hat{\boldsymbol{k}})\right], \quad \lambda=(+, \times)
$$

onde a parte $\ell=m=0$ da soma corresponde à contribuição isotrópica do espectro. As três funções $f_{\mathcal{R}}(k)$ e $f_{\lambda}(k)$ representam a média dos espectros de potências sobre as três direções espaciais

$$
f_{X}(k)=\int \mathcal{P}_{X}(\boldsymbol{k}) \frac{\mathrm{d}^{2} \Omega_{\hat{\boldsymbol{k}}}}{4 \pi}, \quad X=\mathcal{R},+, \times .
$$

Já os três conjuntos de coeficientes, $r_{\ell m}(k)$ e $r_{\ell m}^{\lambda}(k)$, caracterizam o desvio de uma estatística isotrópica. A consistência do nosso modelo requer que a teoria isotrópica seja recuperada em pequenas escalas. Para isso, esses coeficientes devem ser tais que

$$
r_{\ell m}(k) \rightarrow 0 \quad \text { quando } \quad \frac{k}{k_{\text {ref }}} \gg 1, \ell \gg 1,
$$


e

$$
r_{\ell m}^{\lambda}(k) \rightarrow 0 \quad \text { quando } \quad \frac{k}{k_{\text {ref }}} \gg 1, \ell \gg 1 .
$$

Portanto, da discussão anterior, concluímos que existem apenas $1+\ell / 2$ coeficientes independentes da forma

$$
\left(r_{\ell m}, r_{\ell m}^{\lambda}\right) \in \mathbb{R} \quad \ell=2 \ell^{\prime}, m=2 m^{\prime}, \quad m^{\prime}=0 \ldots \ell^{\prime}
$$

\section{7 $\quad$ Previsões}

Para ilustrarmos as assinaturas do período inflacionário anisotrópico, consideraremos um modelo específico com $\alpha=\pi / 4$. Fixaremos o valor inicial do inflaton de tal sorte que $\varphi_{0} / M_{\mathrm{p}}=3.25 \sqrt{8 \pi} \simeq 16.3$. Isso implica que o número de $e$-folds do período inflacionário é $N\left[\varphi_{i}\right] \simeq 67$. Para o número de onda $k_{\text {ref }}$ tomaremos $k_{\text {ref }} \simeq 157 m \simeq$ $15710^{-6} M_{\mathrm{p}}$. Isso corresponde à definição (7.27) calculada com a solução aproximada de constante cosmológica (5.40) e (5.45) e com a expressão (5.66) para o valor de $\tau_{*}$.

Os detalhes técnicos utilizados no cálculo numérico das perturbações e, consequentemente, dos espectros de potências, estão detalhados no Apêndice B. Para que possamos entender o comportamento dos espectros, apresentamos:

- Fig. 7.9: evolução das funções $f_{\mathcal{R}}(k)$ e $f_{\lambda}(k)$ como função de $k$ para modos variando de $3 k_{\text {ref a }} 100 k_{\text {ref. }}$ Esta figura mostra a evolução da parte isotrópica que domina as pequenas escalas.

A partir desta figura concluímos que o espectro de potências associado à perturbação de curvatura tem um índice espectral $n_{s}-1 \simeq-0.032$. Para o modelo definido com os valores acima, temos que $\delta \ll \epsilon$. Assim os modos apresentados se tornam maiores que o raio de Hubble quando $\epsilon$ é aproximadamente constante e vale $\epsilon \sim 0.008$ (veja a Fig. 5.6). O valor esperado para o índice espectral durante a fase inflacionária isotrópica é, portanto, da ordem de $n_{s}-1=2 \delta-4 \epsilon \sim-0.032$, em completo acordo com nossos resultados numéricos.

No que diz respeito aos modos tensoriais, vemos claramente que $P_{+}$e $P_{\times}$diferem em grandes escalas e convergem ao espectro isotrópico da inflação padrão em pequenas escalas. Em particular, podemos verificar que $n_{T}=-2 \epsilon \sim-0.016$, em perfeito acordo com nossos cálculos numéricos. A relação de consistência, definida em (4.47), nos diz que $r \simeq 0.016$. Esse valor está dentro do limite observacional, $r<0.2$, imposto pelo satélite WMAP [9].

- Fig. 7.10: projeção Mollweide de $\mathcal{P}_{\mathcal{R}}(\boldsymbol{k})$ para diferentes números de onda. Esta figura apresenta uma confirmação visual da isotropização em pequenas escalas.

- Fig. 7.11: $r_{\ell m}$ e $r_{\ell m}^{\lambda}$ como função de $k$ para os multipolos mais baixos $(\ell=2)$. Novamente vemos a isotropização em pequenas escalas, como na figura anterior.

É importante enfatizar que as previsões acima podem ser geradas para qualquer espaçotempo do tipo Bianchi I (ou seja, qualquer valor de $\alpha$ ) e para qualquer multipolo $\ell$. Apresentamos no Apêndice B as previsões do caso particular $\alpha=\pi / 2$ considerado em [23]. 

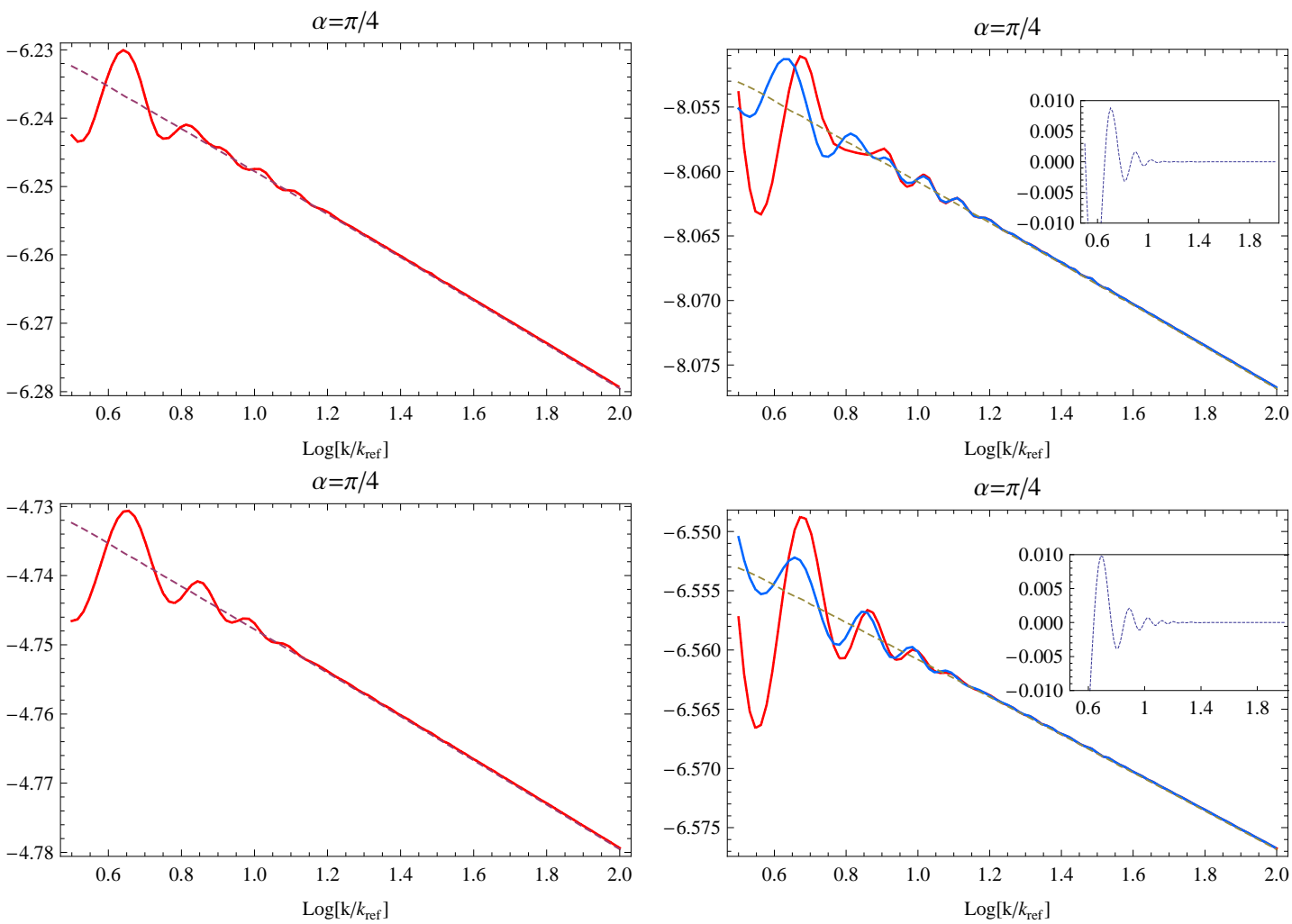

Figura 7.9: Evolução das funções $\log \left[f_{\mathcal{R}}(k)\right]$ (esquerda) e $\log \left[f_{\lambda}(k)\right]$ (direita) para as duas polarizações das ondas gravitacionais em termos de $\log \left[k / k_{\text {ref }}\right]$, com $\varphi_{0}=16.3 M_{\mathrm{p}}$. O caso isotrópico (FRW) está representado pela curva tracejada. A figura interna à direita representa a diferença relativa entre as polarizações $+\mathrm{e} \times$, o que mostra que, em pequenas escalas, recuperamos o resultado isotrópico $P_{\times}=P_{+}$. As figuras superiores foram geradas a partir de condições iniciais definidas em $t_{i}=t_{i}(\boldsymbol{k})$ e as inferiores em $t_{i}=\tau_{*}$. Vemos também que para $\log \left(k / k_{\text {ref }}\right) \gtrsim 0.8$ os espectros são idênticos. Para números de onda muito menores que $k_{\text {ref }}$ a aproximação WKB não é boa o suficiente para nos permitir fixar, sem ambiguidades, as condições iniciais. 

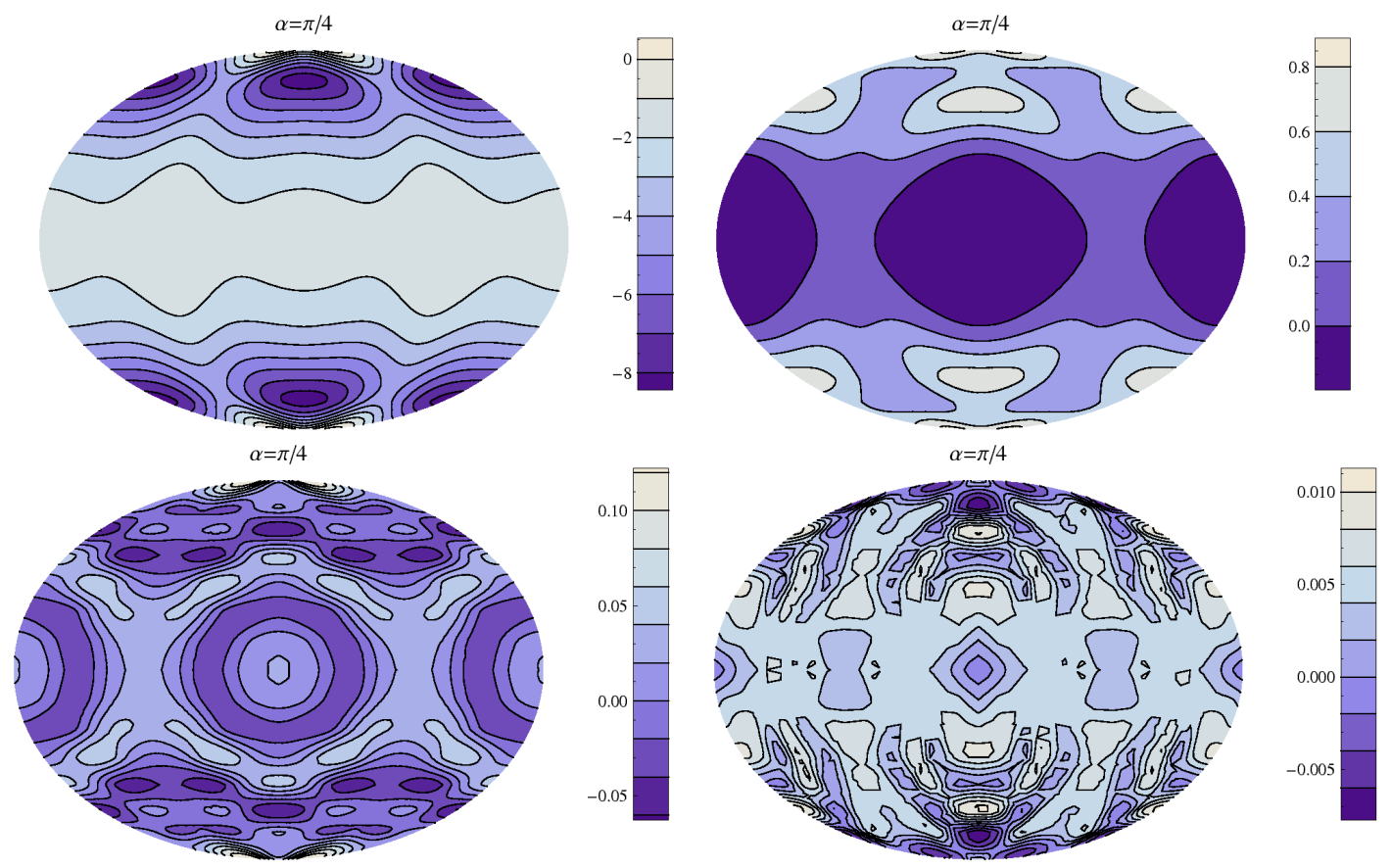

Figura 7.10: Projeção Mollweide da razão entre $\mathcal{P}_{\mathcal{R}}(\boldsymbol{k})$ e seu análogo isotrópico, expressa em porcentagem, para $\log \left[k / k_{\text {ref }}\right]=1 / 2,1,3 / 2,2$ respectivamente da esquerda para a direita e de cima para baixo.
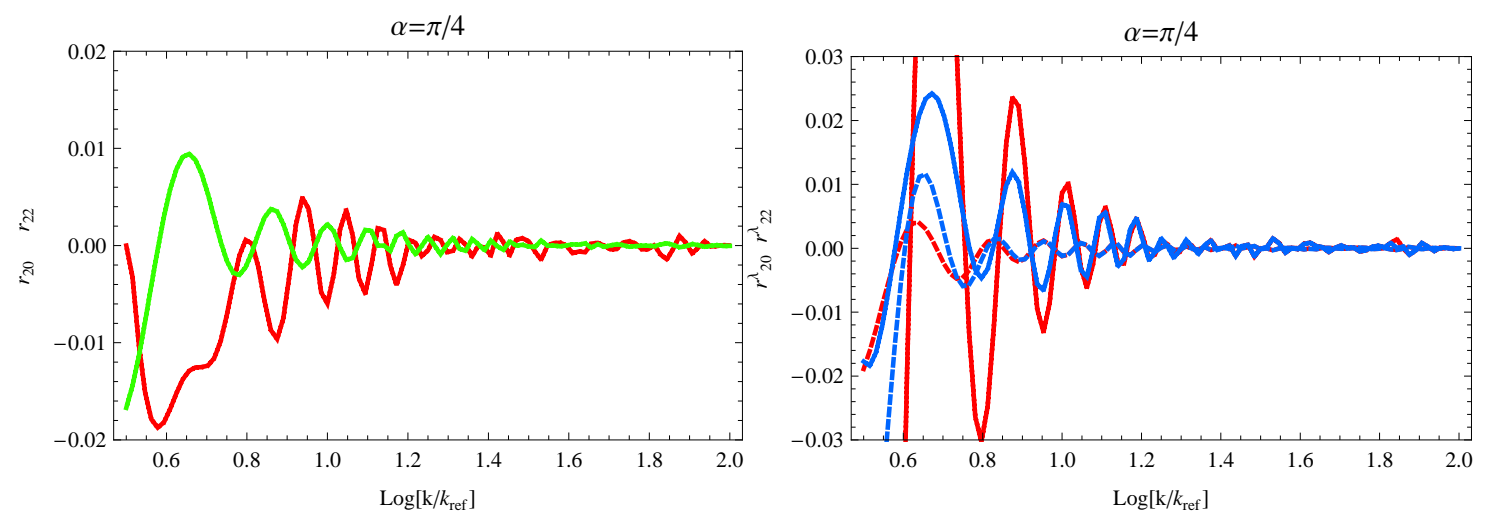

Figura 7.11: Evolução dos coeficientes $r_{\ell m}(k)$ (esquerda) e $r_{\ell m}^{\lambda}(k)$ (direita) como função de $\log \left[k / k_{\text {ref }}\right]$ para $\ell=2$. Os momentos $m=0$ e $m=2$ são apresentados respectivamente em vermelho e verde à esquerda. À direita os momentos $m=0$ e $m=2$ são representados (respectivamente) em linhas contínuas e tracejadas, a vermelha correspondendo à polarização + e a azul à polarização $\times$. 


\section{CAPÍTULO 8}

\section{Conclusões Finais e Perspectivas}

Desenvolvemos neste trabalho as previsões genéricas de uma fase inflacionária e anisotrópica para um espaço-tempo do tipo Bianchi I. Apresentamos uma descrição detalhada do processo de isotropização do universo em seus aspectos homogêneos e linearmente perturbados, bem como uma descrição da evolução dinâmica dos modos perturbativos do universo nesse contexto.

De modo geral, os espaços-tempo de Bianchi possuem uma direção ricocheteante, exceto no caso singular $\alpha=\pi / 2$ considerado em [23]. O caso $\alpha=\pi / 2$ é especialmente singular se levarmos em conta que, durante o regime de domínio do cisalhamento, os invariantes da métrica (equações (5.51-5.53)) avaliados em torno da singularidade não possuem uma convergência uniforme em torno de $\alpha=\pi / 2$. Dado que nos instantes iniciais de evolução os modos perturbativos não possuem um regime WKB, fomos forçados a estender o procedimento padrão de definição das condições iniciais. Mostramos que os modos maiores que $k_{\text {ref }}$ sempre passam por um regime WKB antes de se tornarem maiores que o raio de Hubble. Nosso procedimento leva a resultados que reproduzem o modelo isotrópico das perturbações cosmológicas em pequenas escalas.

Através de uma análise numérica, apresentamos o impacto da fase anisotrópica primordial sobre o espectro de potências das perturbações escalares e tensoriais no fim da inflação. Nossos resultados devem ser interpretados levando em conta que o cisalhamento primordial deve ser ajustado de modo a permitir uma definição precisa das condições iniciais. De fato, mostramos que existe apenas uma pequena janela onde é possível quantizar de maneira precisa os modos que carregam consigo a anisotropia do espaço. Os resultados apresentados referem-se a dois universos particulares $\alpha=$ $\pi / 4$ e $\alpha=\pi / 2$ (detalhes no Apêndice $\mathrm{C}$ ), entretanto a generalização dos resultados apresentados para um valor arbitrário de $\alpha$ é imediata.

No que diz respeito às condições iniciais, temos dois problemas específicos a considerar. Em primeiro lugar, durante a fase anisotrópica primordial, a aproximação WKB é violada. Isso nos impede de definir de maneira precisa as condições iniciais das perturbações, como é feito em universos de FRW. Como mostramos, os modos com comprimentos de onda menores que o raio de Hubble durante o inicio da inflação podem ser quantizados pois as flutuações quânticas, por hipótese, agem o tempo todo e 
podem sempre "alimentar" as soluções oscilantes nesse regime. Por outro lado, devido à não trivialidade do espaço-tempo de fundo, sempre existirá soluções não-oscilantes (para as quais $\omega_{v, \lambda}^{2}<0$ ). Contudo, tais modos devem ser iguais ou maiores que o raio de Hubble hoje, dado que uma assinatura não-trivial de anisotropia na RCF ainda não foi observada. Isso significa que existe uma escala de corte acima da qual não podemos prever os espectros de potências a partir de primeiros princípios segundo a prescrição por nós adotada. Conseqüentemente, acima dessas escalas nós podemos apenas medir o espectro de potências ou postular sua forma inicial, como de fato era feito antes do advento da teoria inflacionária. Em segundo lugar, modos com grandes comprimentos de onda não são independentes uns dos outros. Isso significa que a quantização correta nesse regime envolve três campos interagentes, o que dificulta bastante o acesso aos observáveis. Modelos análogos de campos auto-interagentes foram estudados nas referências [25, 26, 103, 104, 105], onde os observáveis de interesse eram correlações não-gaussianas entre os campos. Contudo, nosso caso é diferente pois as interações envolvem acoplamentos entre os diferentes campos e não acoplamentos de auto-interação. Até o presente momento a quantização completa desses modelos ainda não foi abordada na literatura.

Nossa análise depende também da validade da relatividade geral até os instantes próximos à singularidade inicial. Evidentemente, não pretendemos extrapolar o modelo para além de seus limites, nem assumir sua validade em escalas acima da escala de Planck. Nesse regime, espera-se que graus de liberdade adicionais possam alterar de modo significativo a dinâmica do universo. De fato, é possível encontrar exemplos de uma dinâmica primordial em vários contextos, como por exemplo através de termos de campos axiônicos do tipo Kalb-Ramond [101], modelos com geometrias não-comutativas [102] e, recentemente, nos chamados modelos de loop quantum gravity [106]. Em princípio, o subseqüente desenvolvimento de qualquer um desses modelos pode levar a uma descrição precisa de um período primordial do universo com a correta prescrição das suas condições iniciais.

Ainda no que diz respeito à inflação, nosso estudo demonstra até que ponto as previsões do modelo padrão são sensíveis às anisotropias (clássicas) de grandes comprimentos de onda. Na presença de anisotropia, mostramos que é impossível definir-se um estado de vácuo de Bunch-Davies da maneira padrão. Todavia, se ajustarmos as condições iniciais de modo que o número de $e$-folds seja suficientemente grande, nenhum dos problemas aqui apresentados afetará os modos hoje observáveis, pois se $N \gg 70$, apenas os modos tais que $k / k_{\text {ref }} \gg 1$ estarão acessíveis aos nossos instrumentos; porém, como vimos, nesse limite nós recuperamos as previsões inflacionárias usuais. Se esse for o caso, nenhuma assinatura de anisotropia primordial pode em princípio ser detectada. Nossa análise e conclusões nesse caso podem ser de alguma relevância para a construção de modelos inflacionários no contexto de teorias de cordas se a hipótese de modelos de "campos pequenos" e "inflação curta" (short inflation) persistir, questão essa que está fora do escopo do presente trabalho.

Se a indicação de uma quebra de isotropia estatística da RCF for confirmada e relacionada à uma fase anisotrópica primordial, então uma nova coincidência surgirá nos modelos cosmológicos, uma vez que será necessário entender o motivo pela qual a escala característica da inflação é da ordem do raio de Hubble hoje, ou seja, $k_{\text {ref }} \sim k_{0}$. Entretanto, de um ponto de vista mais pragmático, o presente trabalho nos permite extrair da RCF sinais da fase anisotrópica. Nesse sentido, é importante enfatizar que 
a forma dos espectros de potências aqui apresentados é mais geral do que aquelas heuristicamente apresentadas em $[17,24]$ e generalizam a expressão dada para o caso singular $\alpha=\pi / 2$ estudado em [23]. Além disso, a existência de correlação entre ondas gravitacionais e a perturbação de curvatura, bem como o fato de que as duas polarizações das ondas gravitacionais não dividem o mesmo espectro, podem também levar a assinaturas específicas a serem investigadas.

Com relação às perspectivas de desenvolvimento deste trabalho, existem dois pontos particularmente interessantes que pretendemos analisar. O primeiro deles diz respeito aos vínculos observacionais compatíveis com uma fase anisotrópica primordial. Em outras palavras, será interessante conhecer o valor superior do cisalhamento primordial impostos pelos dados da RCF. Essa tarefa já pode ser conduzida com ajuda dos últimos dados do satélite WMAP [107] e, em breve, com os resultados do satélite Planck [108]. Além disso, a função de correlação (7.48) nos mostra que mesmo a parte isotrópica do espectro de temperatura pode receber uma contribuição do espectro anisotrópico. Isso nos leva a crer que a modulação do parâmetro $\alpha$, característico dos modelos de Bianchi I, pode levar a alguma modulação observável dos baixos multipolos da RCF.

Uma segunda questão está relacionada às regras de seleção (7.51) que resultam das simetrias do espaço-tempo de fundo. É curioso notar que, diferentemente do que tem sido argumentado na literatura, a quebra de simetria rotacional do universo não é capaz de correlacionar momentos multipolares separados por "distâncias" ímpares. Em particular, a anisotropia do espaço é incapaz de correlacionar os termos de quadrupolo e octopolo da RCF, o que supostamente explicaria a origem do chamado "eixo do mal" [78]. Por outro lado uma quebra de invariância translacional poderia levar a tais correlações. Um estudo aprofundado dessa questão certamente merece ser desenvolvido, dado que o estabelecimento de relações precisas entre as simetrias do universo e as correlações angulares da RCF poderia restringir de maneira considerável a degenerescência dos modelos cosmológicos. 
[1] T. S. Pereira, C. Pitrou, and J.-P. Uzan, JCAP 0709, 006 (2007), arXiv:0707.0736 [astro-ph].

[2] C. Pitrou, T. S. Pereira, and J.-P. Uzan, JCAP 0804, 004 (2008), 0801.3596.

[3] E. Hubble, Proc. Nat. Acad. Sci. 15, 168 (1929).

[4] A. A. Penzias and R. W. Wilson, Astrophys. J. 142, 419 (1965).

[5] J. C. Mather et al., Astrophys. J. 354, L37 (1990).

[6] WMAP, C. L. Bennett et al., Astrophys. J. Suppl. 148, 1 (2003), astro$\mathrm{ph} / 0302207$.

[7] WMAP, D. N. Spergel et al., Astrophys. J. Suppl. 170, 377 (2007), astro$\mathrm{ph} / 0603449$.

[8] WMAP, G. Hinshaw et al., (2008), 0803.0732.

[9] WMAP, E. Komatsu et al., (2008), 0803.0547.

[10] A. H. Guth, Phys. Rev. D23, 347 (1981).

[11] M. Tegmark, A. de Oliveira-Costa, and A. Hamilton, Phys. Rev. D68, 123523 (2003), astro-ph/0302496.

[12] G. Efstathiou, Mon. Not. Roy. Astron. Soc. 348, 885 (2004), astro-ph/0310207.

[13] A. de Oliveira-Costa, M. Tegmark, M. Zaldarriaga, and A. Hamilton, Phys. Rev. D69, 063516 (2004), astro-ph/0307282.

[14] D. J. Schwarz, G. D. Starkman, D. Huterer, and C. J. Copi, Phys. Rev. Lett. 93, 221301 (2004), astro-ph/0403353.

[15] C. J. Copi, D. Huterer, and G. D. Starkman, Phys. Rev. D70, 043515 (2004), astro-ph/0310511. 
[16] H. K. Eriksen, A. J. Banday, K. M. Gorski, and P. B. Lilje, Astrophys. J. 612, 633 (2004), astro-ph/0403098.

[17] A. R. Pullen and M. Kamionkowski, Phys. Rev. D76, 103529 (2007), arXiv:0709.1144 [astro-ph].

[18] L. R. Abramo and J. Sodre, Laerte, (2003), astro-ph/0312124.

[19] L. R. Abramo, L. S. Jr., and C. A. Wuensche, Phys. Rev. D74, 083515 (2006), astro-ph/0605269.

[20] A. Bernui and W. S. Hipolito-Ricaldi, (2008), 0807.1076.

[21] J. P. Luminet, J. Weeks, A. Riazuelo, R. Lehoucq, and J. P. Uzan, Nature. 425, 593 (2003), astro-ph/0310253.

[22] B. Mota, G. I. Gomero, M. J. Reboucas, and R. Tavakol, Class. Quant. Grav. 21, 3361 (2004), astro-ph/0309371.

[23] A. E. Gumrukcuoglu, C. R. Contaldi, and M. Peloso, JCAP 0711, 005 (2007), arXiv:0707.4179 [astro-ph].

[24] L. Ackerman, S. M. Carroll, and M. B. Wise, Phys. Rev. D75, 083502 (2007), astro-ph/0701357.

[25] S. Weinberg, Phys. Rev. D72, 043514 (2005), hep-th/0506236.

[26] S. Weinberg, Phys. Rev. D74, 023508 (2006), hep-th/0605244.

[27] N. Bartolo, E. Komatsu, S. Matarrese, and A. Riotto, Phys. Rept. 402, 103 (2004), astro-ph/0406398.

[28] J. Yadav, S. Bharadwaj, B. Pandey, and T. R. Seshadri, Mon. Not. Roy. Astron. Soc. 364, 601 (2005), astro-ph/0504315.

[29] http://www.sdss.org/.

[30] S. Weinberg, Gravitation and Cosmology (Wiley, 1972).

[31] M. J. Reboucas, AIP Conf. Proc. 782, 188 (2005), astro-ph/0504365.

[32] W. Rindler, Gen. Rel. Grav. 34, 133 (2002).

[33] R. A. Alpher, H. Bethe, and G. Gamow, Phys. Rev. 73, 803 (1948).

[34] G. Gamow, Nature 162, 680 (1948).

[35] R. A. Alpher and R. Herman, Nature 162, 774 (1948).

[36] W. L. Freedman, Phys. Rept. 333, 13 (2000), astro-ph/9909076.

[37] S. Weinberg, Cosmology (Oxford University Press, USA, 2008).

[38] S. W. Hawking, I. G. Moss, and J. M. Stewart, Phys. Rev. D26, 2681 (1982). 
[39] A. D. Linde, Phys. Lett. B108, 389 (1982).

[40] A. Albrecht and P. J. Steinhardt, Phys. Rev. Lett. 48, 1220 (1982).

[41] A. Linde, Lect. Notes Phys. 738, 1 (2008), arXiv:0705.0164 [hep-th].

[42] V. F. Mukhanov and G. V. Chibisov, JETP Lett. 33, 532 (1981).

[43] S. W. Hawking, Phys. Lett. B115, 295 (1982).

[44] A. A. Starobinsky, Phys. Lett. B117, 175 (1982).

[45] J. M. Bardeen, P. J. Steinhardt, and M. S. Turner, Phys. Rev. D28, 679 (1983).

[46] A. D. Linde, Phys. Lett. B129, 177 (1983).

[47] E. R. Harrison, Phys. Rev. D1, 2726 (1970).

[48] Y. B. Zeldovich, Mon. Not. Roy. Astron. Soc. 160, 1P (1972).

[49] H. Kodama and M. Sasaki, Prog. Theor. Phys. Suppl. 78, 1 (1984).

[50] V. F. Mukhanov, H. A. Feldman, and R. H. Brandenberger, Phys. Rept. 215, 203 (1992).

[51] J. M. Bardeen, Phys. Rev. D22, 1882 (1980).

[52] J. M. Stewart and M. Walker, Proc. Roy. Soc. Lond. A341, 49 (1974).

[53] K. Tomita and M. Den, Phys. Rev. D34, 3570 (1986).

[54] P. Peter and J.-P. Uzan, Cosmologie Primordiale (Belin, 2005).

[55] D. H. Lyth, Phys. Rev. D31, 1792 (1985).

[56] M. Sasaki, Prog. Theor. Phys. 76, 1036 (1986).

[57] V. F. Mukhanov, Sov. Phys. JETP 67, 1297 (1988).

[58] R. Arnowitt, S. Deser, and C. W. Misner, (1962), gr-qc/0405109.

[59] N. D. Birrell and P. C. W. Davies, Cambridge, Uk: Univ. Pr. (1982) 340p.

[60] J. Martin, Lect. Notes Phys. 738, 193 (2008), 0704.3540.

[61] V. F. Mukhanov, JETP Lett. 41, 493 (1985).

[62] G. F. R. Ellis, S. J. Stoeger, William R., P. McEwan, and P. Dunsby, Gen. Rel. Grav. 34, 1445 (2002), gr-qc/0109023.

[63] J.-P. Uzan, U. Kirchner, and G. F. R. Ellis, Mon. Not. Roy. Astron. Soc. 344, L65 (2003), astro-ph/0302597.

[64] A. K. Raychaudhuri and B. Modak, Class. Quant. Grav. 5, 225 (1988).

[65] T. Rothman and G. F. R. Ellis, Phys. Lett. B180, 19 (1986). 
[66] D. S. Goldwirth and T. Piran, Phys. Rev. Lett. 64, 2852 (1990).

[67] D. S. Goldwirth and T. Piran, Phys. Rev. D40, 3263 (1989).

[68] O. Iguchi and H. Ishihara, Phys. Rev. D56, 3216 (1997), gr-qc/9611047.

[69] N. Deruelle and D. S. Goldwirth, Phys. Rev. D51, 1563 (1995), gr-qc/9409056.

[70] C. B. Collins and S. W. Hawking, Astrophys. J. 180, 317 (1973).

[71] R. W. Wald, Phys. Rev. D28, 2118 (1983).

[72] A. B. Burd and J. D. Barrow, Nucl. Phys. B308, 929 (1988).

[73] R. Maartens, V. Sahni, and T. D. Saini, Phys. Rev. D63, 063509 (2001), grqc/0011105.

[74] B. C. Paul, Phys. Rev. D64, 124001 (2001), gr-qc/0107005.

[75] M. G. Santos, F. Vernizzi, and P. G. Ferreira, Phys. Rev. D64, 063506 (2001), hep-ph/0103112.

[76] Y. B. Zeldovich and A. A. Starobinsky, Sov. Phys. JETP 34, 1159 (1972).

[77] H. K. Eriksen, F. K. Hansen, A. J. Banday, K. M. Gorski, and P. B. Lilje, Astrophys. J. 605, 14 (2004), astro-ph/0307507.

[78] K. Land and J. Magueijo, Phys. Rev. Lett. 95, 071301 (2005), astro-ph/0502237.

[79] C. Armendariz-Picon, JCAP 0709, 014 (2007), arXiv:0705.1167 [astro-ph].

[80] A. E. Gumrukcuoglu, C. R. Contaldi, and M. Peloso, (2006), astro-ph/0608405.

[81] A. Riazuelo, J.-P. Uzan, R. Lehoucq, and J. Weeks, Phys. Rev. D69, 103514 (2004), astro-ph/0212223.

[82] J.-P. Uzan, A. Riazuelo, R. Lehoucq, and J. Weeks, Phys. Rev. D69, 043003 (2004), astro-ph/0303580.

[83] S. Prunet, J.-P. Uzan, F. Bernardeau, and T. Brunier, Phys. Rev. D71, 083508 (2005), astro-ph/0406364.

[84] T. R. Jaffe, A. J. Banday, H. K. Eriksen, K. M. Gorski, and F. K. Hansen, Astrophys. J. 629, L1 (2005), astro-ph/0503213.

[85] T. R. Jaffe, A. J. Banday, H. K. Eriksen, K. M. Gorski, and F. K. Hansen, (2006), astro-ph/0606046.

[86] R. Maartens, G. F. R. Ellis, and S. J. Stoeger, William R., Astron. Astrophys. 309, L7 (1996), astro-ph/9510126.

[87] S. J. Stoeger, William R., M. Araujo, and T. Gebbie, Astrophys. J. 476, 435 (1997), astro-ph/9904346. 
[88] A. Kogut, G. Hinshaw, and A. J. Banday, Phys. Rev. D55, 1901 (1997), astro$\mathrm{ph} / 9701090$.

[89] G. F. R. Ellis and M. A. H. MacCallum, Commun. Math. Phys. 12, 108 (1969).

[90] A. Borde, A. H. Guth, and A. Vilenkin, Phys. Rev. Lett. 90, 151301 (2003), gr-qc/0110012.

[91] C. Pitrou, Dynamique non-linéaire et anisotropie primordiale en cosmologie, PhD thesis, Institut d'Astrophysique de Paris - Université Paris VI, 2008.

[92] J. Martin and D. J. Schwarz, Phys. Rev. D67, 083512 (2003), astro-ph/0210090.

[93] J. E. Lidsey et al., Rev. Mod. Phys. 69, 373 (1997), astro-ph/9508078.

[94] A. R. Liddle and D. H. Lyth, Phys. Rept. 231, 1 (1993), astro-ph/9303019.

[95] S. Sarkar, Rept. Prog. Phys. 59, 1493 (1996), hep-ph/9602260.

[96] D. Wands, Lect. Notes Phys. 738, 275 (2008), astro-ph/0702187.

[97] C. T. Byrnes and D. Wands, Phys. Rev. D74, 043529 (2006), astro-ph/0605679.

[98] M. Lemoine, M. Lubo, J. Martin, and J.-P. Uzan, Phys. Rev. D65, 023510 (2002), hep-th/0109128.

[99] T. Damour and M. Henneaux, Phys. Lett. B488, 108 (2000), hep-th/0006171.

[100] T. Damour and M. Henneaux, Phys. Rev. Lett. 85, 920 (2000), hep-th/0003139.

[101] N. Kaloper, Phys. Rev. D44, 2380 (1991).

[102] E. Di Grezia, G. Esposito, A. Funel, G. Mangano, and G. Miele, Phys. Rev. D68, 105012 (2003), gr-qc/0305050.

[103] J. M. Maldacena, JHEP 05, 013 (2003), astro-ph/0210603.

[104] F. Bernardeau, T. Brunier, and J.-P. Uzan, Phys. Rev. D69, 063520 (2004), astro-ph/0311422.

[105] T. Brunier, F. Bernardeau, and J.-P. Uzan, Phys. Rev. D71, 063529 (2005), hep-th/0412186.

[106] D.-W. Chiou, Phys. Rev. D76, 124037 (2007), arXiv:0710.0416 [gr-qc].

[107] http://map.gsfc.nasa.gov/.

[108] http://www.rssd.esa.int/index.php?project=planck. 


\section{APÊNDICE A}

Fórmulas Gerais

\section{A.1 Funções de Hankel}

Apresentamos abaixo as expansões assintóticas para as funções de Hankel de primeira e segunda espécies usadas no texto. Definindo $x \equiv-k \eta$, sendo que $k>0$ e $\eta \in(-\infty, 0)$, temos:

Para $x \rightarrow \infty$

$$
H_{\nu}^{(1)}(x) \approx \sqrt{\frac{2}{\pi z}} e^{i\left(x-\nu \frac{\pi}{2}-\frac{\pi}{4}\right)}, H_{\nu}^{(2)} \approx \sqrt{\frac{2}{\pi z}} e^{-i\left(x-\nu \frac{\pi}{2}-\frac{\pi}{4}\right)} .
$$

Para $x \rightarrow 0$

$$
H_{\nu}^{(1)}(x) \approx-i \frac{2^{\nu-1}}{\pi} \frac{\Gamma(\nu)}{\Gamma(3 / 2)} x^{-\nu}, \quad H_{\nu}^{(2)}(x) \approx i \frac{2^{\nu-1}}{\pi} \frac{\Gamma(\nu)}{\Gamma(3 / 2)} x^{-\nu} .
$$

\section{A.2 Decomposição das ondas planas}

A decomposição das ondas planas em funções harmônicas esféricas usadas na dedução da função de correlação (7.44) é

$$
e^{\mathrm{i} \boldsymbol{k} \cdot \boldsymbol{x}}=4 \pi \sum_{\ell=0}^{\infty} \sum_{m=-\ell}^{\ell} \mathrm{i}^{\ell} j_{\ell}(k x) Y_{\ell m}^{*}(\hat{\boldsymbol{k}}) Y_{\ell m}(\hat{\boldsymbol{x}}) .
$$


APÊNDICE B

Detalhes dos Cálculos Algébricos

Apresentaremos aqui alguns detalhes dos principais cálculos algébricos feitos nesse trabalho. Os cálculos exatos baseiam-se na métrica de fundo (5.25) e os cálculos perturbativos partem da métrica (6.29), (6.45) e (6.46), ambas no tempo conforme.

\section{B.1 Equações anisotrópicas de fundo}

Os símbolos de Christoffel não-nulos que resultam da métrica (5.25) são

$$
\Gamma_{00}^{0}=\mathcal{H}, \quad \Gamma_{i j}^{0}=\mathcal{H} \gamma_{i j}+\sigma_{i j}, \quad \Gamma_{0 j}^{i}=\mathcal{H} \delta_{j}^{i}+\sigma_{j}^{i} .
$$

A partir destes símbolos podemos calcular os componentes não-nulos do tensor de Ricci

$$
\begin{aligned}
S^{2} R_{0}^{0} & =3 \mathcal{H}^{\prime}+\sigma^{2}, \\
S^{2} R_{j}^{i} & =\left(\mathcal{H}^{\prime}+2 \mathcal{H}^{2}\right) \delta_{j}^{i}+2 \mathcal{H} \sigma_{j}^{i}+\left(\sigma_{j}^{i}\right)^{\prime},
\end{aligned}
$$

onde, mais uma vez, $\sigma^{2}=\sigma^{i j} \sigma_{i j}$. O escalar de Ricci é dado por

$$
S^{2} R=6\left(\mathcal{H}^{\prime}+\mathcal{H}^{2}\right)+\sigma^{2}
$$

E, finalmente, os componentes não-nulos do tensor de Einstein são

$$
\begin{aligned}
S^{2} G_{0}^{0} & =-3 \mathcal{H}^{2}+\frac{1}{2} \sigma^{2} \\
S^{2} G_{j}^{i} & =-\left(2 \mathcal{H}^{\prime}+\mathcal{H}^{2}+\frac{1}{2} \sigma^{2}\right) \delta_{j}^{i}+2 \mathcal{H} \sigma_{j}^{i}+\left(\sigma_{j}^{i}\right)^{\prime} .
\end{aligned}
$$

Para um fluido geral descrito pelo tensor de energia-momento

$$
T_{\mu \nu}=(\rho+p) u_{\mu} u_{\nu}+p g_{\mu \nu}+\pi_{\mu \nu}
$$


onde $\rho$ é a densidade de energia, $p$ a pressão e $\pi_{\mu \nu}$ o estresse anisotrópico $\left(\pi_{\mu \nu} u^{\mu}=0=\pi_{\mu}^{\mu}\right)$, as expressões (B.5,B.6) implicam nas seguintes equações de Einstein

$$
\begin{aligned}
3 \mathcal{H}^{2} & =\kappa S^{2} \rho+\frac{1}{2} \sigma^{2}, \\
\mathcal{H}^{\prime} & =-\frac{\kappa S^{2}}{6}(\rho+3 p)-\frac{1}{3} \sigma^{2}, \\
\left(\sigma_{j}^{i}\right)^{\prime} & =-2 \mathcal{H} \sigma_{j}^{i}+\kappa S^{2} \tilde{\pi}_{j}^{i} .
\end{aligned}
$$

A equação de conservação da matéria fica determinada por

$$
\rho^{\prime}+3 \mathcal{H}(\rho+p)+\sigma_{i j} \tilde{\pi}^{i j}=0,
$$

onde o componente $i j$ do tensor $\pi_{\mu \nu}$ foi definido como $S^{2} \tilde{\pi}_{i j}$. No caso de um fluido perfeito ou de um campo escalar, temos $\pi_{\mu \nu}=0$.

\section{B.2 Propriedades da decomposição EVT}

A derivada temporal do tensor de polarização $\varepsilon_{i j}^{\lambda}$ definido em (6.15) é dada por

$$
\left(\varepsilon_{i j}^{\lambda}\right)^{\prime}=-\left(\sigma^{k l} \varepsilon_{k l}^{\lambda}\right) P_{i j}-\left(\sigma^{k l} P_{k l}\right) \varepsilon_{i j}^{\lambda}+4 \sigma_{(i}^{k} \varepsilon_{j) k}^{\lambda},
$$

ou, equivalentemente

$$
\left(\varepsilon_{j}^{i \lambda}\right)^{\prime}=-\left(\sigma^{k l} \varepsilon_{k l}^{\lambda}\right) P_{j}^{i}-\left(\sigma^{k l} P_{k l}\right) \varepsilon_{j}^{i \lambda}+2 \sigma_{j}^{k} \varepsilon_{k}^{i \lambda} .
$$

Em termos da decomposição (6.63) do tensor de cisalhamento, temos

$$
\left(\varepsilon_{i j}^{\lambda}\right)^{\prime}=-\sigma_{\mathrm{T} \lambda} P_{i j}+\sigma_{\|} \varepsilon_{i j}^{\lambda}+4 \sigma_{(i}^{k} \varepsilon_{j) k}^{\lambda},
$$

e

$$
\left(\varepsilon_{j}^{i \lambda}\right)^{\prime}=-\sigma_{\mathrm{T} \lambda} P_{j}^{i}+\sigma_{\|} \varepsilon_{j}^{i \lambda}+2 \sigma^{k i} \varepsilon_{j k}^{\lambda} .
$$

A derivada temporal do operador de projeção $P_{i j}$ é dada por

$$
\left(P^{i j}\right)^{\prime}=-2 \sigma_{\mathrm{T}+} \varepsilon_{+}^{i j}+2 \sigma_{\|} P^{i j} .
$$

No processo de decomposição das equações de Einstein, fizemos uso da seguinte expressão

$$
\sigma_{i j} e_{a}^{i} e_{a}^{j}=-\frac{1}{2} \sigma_{\|} \delta_{a b}+\sum_{\lambda} \sigma_{\mathrm{T} \lambda} \mathcal{M}_{a b}^{\lambda}
$$

a partir da qual segue que

$$
\sigma_{i j} P^{i j}=-\sigma_{\|}
$$

Usamos também a contração do tensor de cisalhamento com o tensor de polarização

$$
\sigma_{i l} \varepsilon_{\lambda}^{l j}=-\frac{1}{2} \sigma_{\|} \varepsilon_{i}^{j \lambda}+\sum_{a} \sigma_{\mathrm{v} a} \hat{k}_{i} e_{l}^{a} \varepsilon_{\lambda}^{l j}+\sum_{\mu} \sigma_{\mathrm{T} \mu} \varepsilon_{i l}^{\mu} \varepsilon_{\lambda}^{l j},
$$


que por sua vez implica em

$$
\sigma_{i l} \varepsilon_{\lambda}^{l j} P_{j}^{i}=\sigma_{\mathrm{T} \lambda} \quad \text { e } \quad \sigma_{i l} \varepsilon_{\lambda}^{l j} \varepsilon_{j}^{i \mu}=-\frac{1}{2} \sigma_{\|} \delta_{\mu}^{\lambda} .
$$

Concluindo, as contrações abaixo são úteis na dedução das equações de movimento para os modos tensoriais

$$
\begin{aligned}
e_{i}^{b} e_{a}^{j} \sigma_{j l} \varepsilon^{l i} & =-\frac{1}{2} \sigma_{\|} \mathcal{M}_{a b}^{\lambda}+\frac{1}{2} \delta_{b}^{a} \sigma_{\mathrm{T} \lambda}+\frac{1}{2} \mathcal{N}_{a b}\left(\sigma_{\mathrm{T}+} \delta_{\lambda}^{\times}-\sigma_{\mathrm{T} \times} \delta_{\lambda}^{+}\right) . \\
\sigma_{i l} \varepsilon_{\lambda}^{l j} \sigma_{j p} \varepsilon_{\lambda}^{p i} & =\frac{1}{4} \sigma_{\|}^{2}+\frac{1}{2}\left(\sigma_{\mathrm{T} \lambda}^{2}-\sigma_{\mathrm{T}(1-\lambda)}^{2}\right) \\
\sigma_{i l} \varepsilon_{\lambda}^{l j} \sigma_{j p} \varepsilon_{(1-\lambda)}^{p i} & =\sigma_{\mathrm{T}+} \sigma_{\mathrm{T} \times} \\
\sigma_{i l} \varepsilon_{\lambda}^{l j} \sigma^{i p} \varepsilon_{j p}^{\lambda} & =\frac{1}{4} \sigma_{\|}^{2}+\frac{1}{2}\left(\sigma_{\mathrm{T}+}^{2}+\sigma_{\mathrm{T} \times}^{2}\right)+\frac{1}{2} \sum_{a} \sigma_{\mathrm{v} a}^{2} \\
\sigma_{i l} \varepsilon_{\lambda}^{l j} \sigma^{i p} \varepsilon_{j p}^{(1-\lambda)} & =0 .
\end{aligned}
$$

\section{B.3 Detalhes dos cálculos perturbativos}

A perturbação do tensor de energia-momento do inflaton em primeira ordem é

$$
\begin{aligned}
S^{2} \delta T_{0}^{0} & =\varphi^{\prime 2} \Phi-\varphi^{\prime} \chi^{\prime}-V,_{\varphi} S^{2} \chi \\
S^{2} \delta T_{i}^{0} & =-\partial_{i}\left[\varphi^{\prime} \chi\right], \\
S^{2} \delta T_{i}^{j} & =-\delta_{j}^{i}\left[\varphi^{\prime 2} \Phi-\varphi^{\prime} \chi^{\prime}+V,_{\varphi} S^{2} \chi\right] .
\end{aligned}
$$

Notemos que essas expressões são exatamente as mesmas que obtemos no caso de um universo do tipo FRW. Isso decorre do fato de que $\delta g_{i j}$ não aparece nessas expressões. No calibre newtoniano, os símbolos de Christoffel não-nulos e perturbados em primeira ordem são dados por

$$
\begin{aligned}
\delta \Gamma_{00}^{0} & =\Phi^{\prime}, \quad \delta \Gamma_{0 i}^{0}=\partial_{i} \Phi, \\
\delta \Gamma_{i j}^{0} & =\mathcal{H} h_{i j}+\frac{1}{2} h_{i j}^{\prime}-2 \mathcal{H} \Phi \gamma_{i j}-2 \Phi \sigma_{i j}, \\
\delta \Gamma_{0 j}^{i} & =\frac{1}{2}\left(h_{j}^{i}\right)^{\prime}-\sigma_{k j} h^{k i}+h_{k j} \sigma^{k i}, \\
\delta \Gamma_{j k}^{i} & =\frac{1}{2} \gamma^{l i}\left(\partial_{j} h_{l k}+\partial_{k} h_{j l}-\partial_{l} h_{j k}\right) .
\end{aligned}
$$

Já os componentes do tensor de Ricci perturbados em primeira ordem tomam a seguinte forma

$$
\begin{aligned}
-S^{2} \delta R_{0}^{0}= & \nabla^{2} \Phi+3 \mathcal{H} \Phi^{\prime}+3\left(\Psi^{\prime \prime}+\mathcal{H} \Psi^{\prime}\right)+6 \mathcal{H}^{\prime} \Phi-\gamma_{i j}^{\prime} \partial^{i}\left(E^{j}\right)^{\prime}-\gamma_{i}^{\prime j}\left(E_{j}^{i}\right)^{\prime}(\mathrm{B} .33) \\
& +\frac{1}{2}\left[\left(\frac{\Psi}{\mathcal{H}}\right)^{\prime} \gamma_{i j}^{\prime} \gamma^{\prime i j}+\left(\frac{\Psi}{\mathcal{H}}\right) \gamma_{i j}^{\prime} \gamma^{\prime \prime i j}-\left(\frac{\Psi}{\mathcal{H}}\right) \gamma_{j}^{\prime i} \gamma_{i k}^{\prime} \gamma^{\prime j k}\right]+\frac{1}{2} \Phi \gamma_{i j}^{\prime} \gamma^{\prime i j} \\
-S^{2} \delta R_{i}^{0}= & 2 \mathcal{H} \partial_{i} \Phi+2 \partial_{i} \Psi^{\prime}+\frac{1}{2} \nabla^{2} E_{i}^{\prime}+\frac{1}{2}\left(\gamma^{\prime j k} \gamma_{k i}^{\prime}-\gamma_{i}^{\prime \prime j}\right) \partial_{j}\left(\frac{\Psi}{\mathcal{H}}\right)-\frac{1}{2} \gamma_{k i}^{\prime} \nabla^{2} E^{k} \\
& -\frac{1}{2} \gamma_{i}^{\prime j}\left[\partial_{j} \Phi+\partial_{j}\left(\frac{\Psi}{\mathcal{H}}\right)^{\prime}+3 \partial_{j} \Psi\right]+\frac{1}{4} \gamma^{\prime j k} \gamma_{j k}^{\prime} \partial_{i}\left(\frac{\Psi}{\mathcal{H}}\right)-\frac{1}{2} \gamma^{\prime j k} \partial_{i} E_{j k} \\
& +\gamma^{\prime j k} \partial_{j} E_{k i},
\end{aligned}
$$




$$
\begin{aligned}
\delta R_{i j}= & \partial_{i} \partial_{j}(\Psi-\Phi)+\gamma_{i j} \nabla^{2} \Psi-\Phi^{\prime} \mathcal{H} \gamma_{i j}-5 \Psi^{\prime} \mathcal{H} \gamma_{i j}-\left(4 \mathcal{H}^{2}+2 \mathcal{H}^{\prime}\right)(\Phi+\Psi) \gamma_{i j}-\Psi^{\prime \prime} \gamma_{i j} \\
& +\left(4 \mathcal{H}^{2}+2 \mathcal{H}^{\prime}\right)\left[\partial_{(i} E_{j)}+E_{i j}\right]+2 \mathcal{H}\left[\partial_{i} E_{j)}^{\prime}+E_{i j}^{\prime}\right]+\partial_{(i} E_{j)}^{\prime \prime}+E_{i j}^{\prime \prime}-\nabla^{2} E_{i j} \\
& +\frac{1}{2} \gamma_{i j}^{\prime}\left[\frac{1}{\mathcal{H}} \nabla^{2}-\Phi^{\prime}+\Psi\left(\frac{\mathcal{H}^{\prime}}{\mathcal{H}^{2}}\right)^{\prime}-\mathcal{H}\left(\frac{\Psi^{\prime}}{\mathcal{H}^{2}}\right)^{\prime}\right]-\frac{1}{\mathcal{H}} \gamma_{k i}^{\prime} \partial_{j)} \partial^{k} \Psi-4 \mathcal{H} \Psi \gamma_{i j}^{\prime} \\
& +\left(\gamma_{j}^{\prime k} \gamma_{k i}^{\prime}-\gamma_{i j}^{\prime \prime}-2 \mathcal{H} \gamma_{i j}^{\prime}\right) \Phi-\frac{5}{2} \Psi^{\prime} \gamma_{i j}^{\prime}-\frac{\Psi^{\prime}}{\mathcal{H}}\left(\gamma_{i j}^{\prime \prime}-\gamma_{k i}^{\prime} \gamma_{j}^{\prime k}\right)-2 \Psi \gamma_{i j}^{\prime \prime} \\
& +\Psi \frac{\mathcal{H}^{\prime}}{\mathcal{H}^{2}}\left(\gamma_{i j}^{\prime \prime}-\gamma_{k i}^{\prime} \gamma_{j}^{\prime k}\right)+\frac{\Psi}{2 \mathcal{H}}\left(-\gamma_{i j}^{\prime \prime \prime}+2 \mathcal{H} \gamma_{i}^{\prime k} \gamma_{k j}^{\prime}+2 \gamma_{(j}^{\prime k} \gamma_{k i)}^{\prime \prime}-\gamma^{\prime k l} \gamma_{k i}^{\prime} \gamma_{l j}^{\prime}\right) \\
& -\left(\gamma_{i}^{\prime k} \partial_{(k} E_{j)}^{\prime}+\gamma_{j}^{\prime k} \partial_{(k} E_{i)}^{\prime}\right)+\gamma_{i}^{\prime k} \gamma_{j}^{\prime l} \partial_{(k} E_{l)}-2 \gamma_{(j}^{\prime k} E_{k i)}^{\prime}+\gamma_{i}^{\prime k} \gamma_{j}^{\prime l} E_{k l} .
\end{aligned}
$$

O componente misto do tensor de Ricci espacial, $\delta R_{j}^{i}$, pode ser obtido através de

$$
S^{2} \delta R_{j}^{i}=\gamma^{i k} \delta R_{k j}-\left[-2 \Psi\left(\gamma^{i k}+\frac{\gamma^{\prime i k}}{2 \mathcal{H}}+2 \partial^{(i} E^{k)}+2 E^{i k}\right)\right] R_{k j} .
$$

sendo que $R_{k j}$ foi calculado em (B.3). Finalmente, as expressões para os componentes do tensor de Einstein perturbados em primeira ordem são

$$
\begin{aligned}
S^{2} \delta G_{0}^{0}= & -2 \nabla^{2} \Psi+6 \mathcal{H} \Psi^{\prime}+2 \sigma^{2} \Psi-\left(\frac{\Psi}{\mathcal{H}}\right)^{\prime} \sigma^{2}+\frac{\sigma^{i j}}{\mathcal{H}} \partial_{i} \partial_{j} \Psi \\
& -\sigma^{i j} \partial_{i} \Phi_{j}+\left(E_{j}^{i}\right)^{\prime} \sigma_{i}^{j}+\left(6 \mathcal{H}^{2}-\sigma^{2}\right) \Phi \\
S^{2} \delta G_{i}^{0}= & -\sigma^{2} \frac{\partial_{i} \Psi}{\mathcal{H}}+\sigma_{i}^{j} \partial_{j}\left[\Phi+\Psi+\left(\frac{\Psi}{\mathcal{H}}\right)^{\prime}\right]-2 \partial_{i}\left(\Psi^{\prime}+\mathcal{H} \Phi\right) \\
& +\frac{1}{2} \nabla^{2} \Phi_{i}-2 \sigma^{j k} \partial_{j} E_{i k}+\sigma^{j k} \partial_{i} E_{j k}, \\
S^{2} \delta G_{j}^{i}= & \delta_{j}^{i}\left[2 \Psi^{\prime \prime}+\left(2 \mathcal{H}^{2}+4 \mathcal{H}^{\prime}\right) \Phi+\nabla^{2}(\Phi-\Psi)+2 \mathcal{H} \Phi^{\prime}+4 \mathcal{H} \Psi^{\prime}\right] \\
& +\partial^{i} \partial_{j}(\Psi-\Phi)-\frac{2}{\mathcal{H}} \sigma_{k}^{(i} \partial_{j)} \partial^{k} \Psi+\sigma_{j}^{i}\left[-\mathcal{H}\left(\frac{\Psi^{\prime}}{\mathcal{H}^{2}}\right)^{\prime}+\left(\frac{\mathcal{H}^{\prime}}{\mathcal{H}^{2}}\right)^{\prime} \Psi+\frac{\nabla^{2} \Psi}{\mathcal{H}}-\Phi^{\prime}-\Psi^{\prime}\right] \\
& +\delta_{j}^{i}\left[\sigma^{2}\left(\Phi+\left(\frac{\Psi}{\mathcal{H}}\right)^{\prime}-2 \Psi\right)+\frac{\sigma^{k l}}{\mathcal{H}} \partial_{k} \partial_{l} \Psi\right] \\
& +\left(E^{i}{ }_{j}\right)^{\prime \prime}+2 \mathcal{H}\left(E^{i}{ }_{j}^{\prime}-\nabla^{2} E^{i}{ }_{j}+2\left[\sigma_{k}^{i}\left(E^{k}\right)^{\prime}-\sigma_{j}^{k}\left(E_{k}^{i}\right)^{\prime}\right]-\left[\left(E^{k}\right)^{\prime} \sigma_{k}^{l}\right] \delta_{j}^{i}\right. \\
& +\delta_{j}^{i} \sigma^{k l} \partial_{k} \Phi_{l}-\gamma^{i k}\left[\partial_{(k}\left(\Phi_{j)}\right)^{\prime}+2 \mathcal{H} \partial_{(k} \Phi_{j)}-2 \sigma_{(k}^{l} \partial_{|l|} \Phi_{j)}\right] .
\end{aligned}
$$

\section{B.4 Derivada total na ação}

A derivada total que aparece na ação (6.130) é dada explicitamente por

$$
\begin{aligned}
\mathcal{T}= & {\left[-S^{2} \sigma_{i}^{l} h_{m l} h^{i m}+S^{2} h_{i j} h^{i j} \mathcal{H}+\mathcal{H}(S \delta \varphi)^{2}-\frac{\left(\varphi^{\prime} v\right)^{2}}{2 \mathcal{H}-\sigma_{\|}}-\frac{2 S \varphi^{\prime} v \sigma^{i j} E_{i j}}{2 \mathcal{H}-\sigma_{\|}}\right.} \\
& -\frac{S^{2}\left(\sigma^{i j} E_{i j}\right)^{2}}{2 \mathcal{H}-\sigma_{\|}}-\mathcal{H} \mu_{i j} \mu_{k l} \gamma^{i k} \gamma^{j l}-4 S^{2} \sigma^{j k} E_{i k} \Psi\left(\gamma_{j}^{i}+\frac{\sigma_{j}^{i}}{\mathcal{H}}\right)+\frac{\left(2 \mathcal{H}-\sigma_{\|}\right) S^{2} k^{2} \Psi^{2}}{\mathcal{H}^{2}} \\
& \left.-18 \Psi^{2} \mathcal{H} S^{2}+\frac{7 \Psi^{2} \sigma^{2} S^{2}}{\mathcal{H}}+\frac{2 \Psi^{2} S^{2} \sigma_{i}^{j} \sigma_{j}^{k} \sigma_{k}^{i}}{\mathcal{H}^{2}}-\frac{2 v S \Psi \varphi^{\prime \prime}}{\mathcal{H}}-6 \Psi \varphi^{\prime} S v\right]^{\prime}
\end{aligned}
$$




\section{APÊNDICE C}

\section{Detalhes dos Cálculos Numéricos}

Apresentaremos aqui os detalhes algébricos utilizados na implementação numérica do sistema de equações (6.100-6.101).

Estamos interessados inicialmente em quantificar a evolução temporal de um dado modo $\boldsymbol{k}$ e dos componentes do tensor de cisalhamento $\sigma_{i j}$, referente ao mesmo modo $\boldsymbol{k}$, durante todo o período anisotrópico e a subseqüente isotropização do universo. Para tanto notemos que os covetores $k_{i}$, que são constantes por definição (veja a discussão abaixo da equação (6.1)), levam a vetores duais dependentes do tempo $k^{i}=\gamma^{i j} k_{j}$ uma vez que a métrica $\gamma_{i j}$ (e portanto sua inversa $\gamma^{i j}$ ) depende do tempo. É por esse motivo também que utilizamos os covetores (e não seus duais) para rotular um modo perturbativo.

Uma vez obtidos $S(t)$ e $\beta(t)$ através das equações de Friedmann, a métrica espacial fica completamente determinada de modo que o módulo do vetor $\boldsymbol{k}$ se exprime na forma

$$
k^{2}(t)=\sum_{i} \frac{k_{i}^{2}}{a_{i}^{2}(t)} .
$$

Será útil também definir vetores unitários através de

$$
\hat{k}_{i}(t)=\frac{k_{i}}{k(t)}, \quad \hat{k}^{i}(t)=\frac{k_{i}}{a_{i}^{2}(t) k(t)} .
$$

\section{C.1 Evolução dos componentes do tensor de cisalha- mento}

A decomposição do tensor de cisalhamento em termos dos vetores de onda $\boldsymbol{k}$ requer a construção de uma base ortonormal $\left\{\boldsymbol{e}_{1}(t), \boldsymbol{e}_{2}(t), \hat{\boldsymbol{k}}(t)\right\}$ sujeita a condição

$$
\boldsymbol{e}_{1}^{\prime}(t) \cdot \boldsymbol{e}_{2}(t)=\boldsymbol{e}_{1}(t) \cdot \boldsymbol{e}_{2}^{\prime}(t),
$$

onde o produto escalar está definido em termos da métrica espacial $\gamma_{i j}$. Em princípio, para extrair o valor inicial do tensor de cisalhamento através das Eqs.(6.64,6.65), 
precisamos apenas determinar essa base em um dado instante inicial, $t_{\text {ini }}$,

$$
\Sigma\left(t_{\text {ini }}\right)=\left(\sigma_{\|}, \sigma_{\mathrm{v} 1}, \sigma_{\mathrm{v} 2}, \sigma_{\mathrm{T} \times}, \sigma_{\mathrm{T}}+\right) .
$$

Dessa forma, para determinarmos o valor de $\Sigma$ para qualquer instante $t>t_{\text {ini }}$, usamos o fato de que a Eq.(5.21) implica em

$$
\begin{aligned}
\sigma_{\|}^{\prime}+2 \mathcal{H} \sigma_{\|} & =-2 \sum_{a} \sigma_{\mathrm{v} a}^{2}, \\
\sigma_{\mathrm{v} a}^{\prime}+2 \mathcal{H} \sigma_{\mathrm{v} a} & =\frac{3}{2} \sigma_{\mathrm{v} a} \sigma_{\|}-\sum_{b, \lambda} \sigma_{\mathrm{v} a} \sigma_{\mathrm{T} \lambda} \mathcal{M}_{a b}^{\lambda}, \\
\sigma_{\mathrm{T} \lambda}^{\prime}+2 \mathcal{H} \sigma_{\mathrm{T} \lambda} & =2 \sum_{a, b} \mathcal{M}_{a b}^{\lambda} \sigma_{\mathrm{v} a} \sigma_{\mathrm{v} b},
\end{aligned}
$$

onde a matriz $\mathcal{M}_{a b}^{\lambda}$, definida como

$$
\mathcal{M}_{a b}^{\lambda} \equiv \varepsilon_{i j}^{\lambda} e_{a}^{i} e_{b}^{j},
$$

é trivialmente simétrica nos índices $a b$. Explicitamente, temos

$$
\mathcal{M}_{a b}^{\lambda}=\frac{1}{\sqrt{2}}\left(\begin{array}{cc}
1 & 0 \\
0 & -1
\end{array}\right) \delta_{+}^{\lambda}+\frac{1}{\sqrt{2}}\left(\begin{array}{ll}
0 & 1 \\
1 & 0
\end{array}\right) \delta_{\times}^{\lambda}, \quad \sum_{a} \mathcal{M}_{a a}=0
$$

Um aspecto interessante do sistema de equações (C.5-C.7) é que o mesmo não depende explicitamente de $k_{i}$. Porém, escolhas diferentes de bases levam a soluções diferentes para este sistema. Uma vez que este sistema tenha sido solucionado nós podemos, em princípio, determinar a dinâmica das funções $\left(\omega_{v}, \omega_{\lambda}, \aleph_{\lambda}, \beth\right)$ que entram nas equações dinâmicas das perturbações cosmológicas. Contudo, na prática, à medida em que $\sigma$ vai se tornando menor que $\mathcal{H}$, pequenas oscilações numéricas nos componentes do tensor de cisalhamento são convertidas em enormes instabilidades numéricas do sistema (C.5C.7). No sentido de evitar essas instabilidades numéricas utilizaremos um segundo método que passaremos a detalhar agora.

\section{C.1.1 Construção Sistemática}

Esse método reside sobre o fato de que as soluções do sistema de Eqs. (C.5-C.7) são, formalmente, dadas por

$$
\sigma_{\|}=\sigma_{i j} \hat{k}^{i} \hat{k}^{j}, \quad \sigma_{\mathrm{v} a}=\sigma_{i j} \hat{k}^{i} e_{a}^{j}, \quad \sigma_{\mathrm{T} \lambda}=\sigma_{i j} \varepsilon_{\lambda}^{i j}
$$

como funções do tempo. Ao invés de solucionar o sistema (C.5-C.7) a partir das condições iniciais, podemos determinar a dinâmica dos componentes do tensor de cisalhamento através da dinâmica dos vetores de base, dos tensores de polarização e da métrica espacial $\gamma_{i j}$. Ou seja, uma vez determinado $\beta_{i}^{\prime}(t)$ em função do tempo, determinamos também os componentes de $\sigma_{i j}$. Para determinar a evolução temporal dos vetores de base $\left\{\boldsymbol{e}_{1}(t), \boldsymbol{e}_{2}(t), \hat{\boldsymbol{k}}\right\}$, partimos de uma base $\left\{\boldsymbol{e}_{x}(t), \boldsymbol{e}_{y}(t), \boldsymbol{e}_{z}(t)\right\}$ alinhada com o eixo ortogonal $x y z$ de coordenadas cartesianas. Em seguida, introduzimos três ângulos de Euler capazes de rotacionar essa base inicial em uma base de orientação arbitrária. Para construir uma matriz de rotação apropriada, lembremos que qualquer 
matriz de rotação sobre um vetor unitário $\hat{\mathbf{n}}$ pode ser construída em termos de rotações infinitesimais da forma

$$
R(\theta / N)=1+\left(\frac{\theta}{N}\right) \mathbf{J} \cdot \hat{\mathbf{n}}=1+\left(\frac{\theta}{N}\right) \gamma^{i j} J_{j} \hat{n}_{i}
$$

onde $\theta / N$ é algum ângulo infinitesimal. As matrizes $J_{j}$ são os geradores do grupo de rotações. Em especial, se quisermos nos restringir às rotações em torno do eixo $z$, então o vetor unitário alinhado com este eixo toma a seguinte forma

$$
\hat{n}_{i}=\left(0,0, e^{\beta_{3}}\right)
$$

e, portanto,

$$
R_{z}(\theta)=\lim _{N \rightarrow \infty}\left(1+e^{-\beta_{3}} J_{z} \frac{\theta}{N}\right)^{N}=\exp \left[\theta e^{-\beta_{3}} J_{z}\right] .
$$

Pode ser mostrado que, para que o produto escalar entre dois vetores seja constante, os geradores devem satisfazer $\left(J_{k}\right)_{i j}=-\epsilon_{k i j}$, onde $\epsilon_{i j k}$ é um pseudo-tensor anti-simétrico e normalizado de tal forma que $\epsilon_{123}=\sqrt{\operatorname{det}\left(\gamma_{i j}\right)}=1$. Uma matriz de rotação em torno do eixo $z$ requer então que os geradores satisfaçam $\left(J_{z}\right)^{i}{ }_{j}=(\gamma)^{i k}\left(J_{z}\right)_{k j}$, de onde podemos deduzir a expressão

$$
\left(J_{z}\right)_{j}^{i}=\left(\begin{array}{ccc}
0 & -e^{-2 \beta_{1}} & 0 \\
e^{-2 \beta_{2}} & 0 & 0 \\
0 & 0 & 0
\end{array}\right) .
$$

Consequentemente, levando em conta o vínculo $\sum_{i} \beta_{i}=0$, qualquer rotação em torno do eixo $z$ pode ser escrita na forma

$$
\left[R_{z}(\theta)\right]_{j}^{i}=\left(\begin{array}{ccc}
\cos (\theta) & -e^{\left(\beta_{2}-\beta_{1}\right)} \sin (\theta) & 0 \\
e^{\left(\beta_{1}-\beta_{2}\right)} \sin (\theta) & \cos (\theta) & 0 \\
0 & 0 & 1
\end{array}\right) .
$$

De modo similar, para uma rotação em torno do eixo $y$, podemos utilizar o gerador

$$
\left(J_{y}\right)_{j}^{i}=\left(\begin{array}{ccc}
0 & 0 & e^{-2 \beta_{1}} \\
0 & 0 & 0 \\
-e^{-2 \beta_{3}} & 0 & 0
\end{array}\right)
$$

para obter

$$
\left[R_{y}(\theta)\right]_{j}^{i}=\left(\begin{array}{ccc}
\cos (\theta) & 0 & e^{\left(\beta_{3}-\beta_{1}\right)} \sin (\theta) \\
0 & 1 & 0 \\
-e^{\left(\beta_{1}-\beta_{3}\right)} \sin (\theta) & 0 & \cos (\theta)
\end{array}\right) .
$$

De maneira semelhante obtém-se a matriz de rotação em torno do eixo $x$.

Os componentes da base $\left\{\boldsymbol{e}_{x}(t), \boldsymbol{e}_{y}(t), \boldsymbol{e}_{z}(t)\right\}$ serão definidos explicitamente como

$$
\left(e_{x}\right)^{i} \equiv\left(\begin{array}{c}
e^{-\beta_{1}} \\
0 \\
0
\end{array}\right), \quad\left(e_{y}\right)^{i} \equiv\left(\begin{array}{c}
0 \\
e^{-\beta_{2}} \\
0
\end{array}\right), \quad\left(e_{z}\right)^{i} \equiv\left(\begin{array}{c}
0 \\
0 \\
e^{-\beta_{3}}
\end{array}\right)
$$


Os três ângulos de Euler $(\alpha, \beta, \gamma)$ podem então ser usados para rotacionar esta base específica com relação aos eixos $x, y$ e $z$. Após essa rotação, teremos uma base de orientação arbitrária dada por

$$
\begin{aligned}
\left(e_{1}\right)^{i} & \equiv R_{z}(\gamma)_{j}^{i} R_{y}(\beta)_{l}^{j} R_{z}(\alpha)_{p}^{l}\left(e_{x}\right)^{p}, \\
\left(e_{2}\right)^{i} & \equiv R_{z}(\gamma)_{j}^{i} R_{y}(\beta)^{j}{ }_{l} R_{z}(\alpha)_{p}^{l}\left(e_{y}\right)^{p}, \\
\left(u_{k}\right)^{i} & \equiv R_{z}(\gamma)_{j}^{i} R_{y}(\beta)_{l}^{j} R_{z}(\alpha)_{p}^{l}\left(e_{z}\right)^{p} .
\end{aligned}
$$

A prescrição que acabamos de descrever é ainda incompleta dado que nosso problema também requer que o covetor $u_{k}$ seja igual ao covetor $\hat{\boldsymbol{k}}$ durante toda a evolução do sistema, ou seja

$$
\left(u_{k}\right)_{i}(t)=\hat{k}_{i}(t), \quad \forall t
$$

Visto que $k_{i}$ não depende do tempo, precisamos determinar os ângulos de Euler em função do tempo de modo que, para dois instantes quaisquer $t$ e $t^{\prime}$ tenhamos $\left[u_{k}(t)\right]_{i}=f\left(t, t^{\prime}\right)\left[u_{k}\left(t^{\prime}\right)\right]_{i}$. Esta condição será satisfeita contanto que as relações

$$
\begin{aligned}
\tan (\gamma) & =\tan \left(\gamma_{f}\right) \exp \left[\left(\beta_{1}-\beta_{2}\right)\right] \\
\tan (\beta) & =\exp \left[\left(\beta_{3}-\beta_{1}\right) \frac{\cos \left(\gamma_{f}\right)}{\cos (\gamma)} \tan \left(\beta_{f}\right)\right],
\end{aligned}
$$

também sejam satisfeitas, onde $\gamma_{f}$ e $\beta_{f}$ são os ângulos que correspondem à direção final equivalente ao instante de isotropização do universo. Adicionalmente, nosso problema também exige que a condição

$$
\left[\left(e_{2}\right)^{i}\right]^{\prime}\left[\left(e_{1}\right)_{i}\right]=\left[\left(e_{1}\right)^{i}\right]^{\prime}\left[\left(e_{2}\right)_{i}\right]
$$

seja satisfeita, o que corresponde à escolha de uma base continuamente (ao longo do tempo) ortogonal. Esta condição, por sua vez, requer que

$$
\alpha^{\prime}=-\cos (\beta) \gamma^{\prime}
$$

Podemos integrar esta equação com a ajuda das equações (C.23) e (C.24). Conseqüentemente, uma vez feita a escolha $\left(\beta_{f}, \gamma_{f}\right)$ de ângulos que descrevem a orientação final de um modo, podemos determinar a tripla $(\alpha(t), \gamma(t), \beta(t))$ de ângulos que selecionará uma base adaptada à decomposição dos modos perturbativos para qualquer instante.

Concluindo, o conjunto de equações (C.21-C.26) fornece uma descrição completa da evolução temporal dos vetores de base necessários para extrair os diferentes componentes do tensor de cisalhamento segundo a equação (C.10), para qualquer instante de tempo.

\section{C.2 Aproximação de Rolagem Lenta}

Apresentaremos aqui as expressões explícitas das quantidades $z_{\mathrm{S}}^{\prime \prime} / z_{\mathrm{S}}, z_{\lambda}^{\prime \prime} / z_{\lambda}, \aleph_{\lambda}$ e $\beth$ em termos dos parâmetros de rolagem lenta. Para tanto, vamos introduzir dois parâmetros auxiliares

$$
w \equiv \frac{\sigma_{\|}}{2 \mathcal{H}}, \quad y_{\lambda} \equiv \frac{\sigma_{\mathrm{T} \lambda}}{\sqrt{6} \mathcal{H}},
$$


em termos dos quais obtemos

$$
\begin{aligned}
\frac{z_{\mathrm{S}}^{\prime \prime}}{z_{\mathrm{S}}}= & \mathcal{H}^{2}\left[2+\epsilon\left\{5-4 \delta+2 \epsilon-\frac{(3-\delta)^{2}}{3-\epsilon}\right\}\right. \\
& \left.+x^{2}\{-3-6 \delta+\epsilon(7+4 \delta-4 \epsilon)\}+2 x^{4}(3-\epsilon)^{2}\right] \\
& -6 x^{2}\left\{\epsilon-\delta+x^{2}(3-\epsilon)\right\}+2 \frac{\epsilon}{\mathcal{H}}\left(1-x^{2}\right)\left(\frac{w}{1-w}\right)^{\prime} \\
\frac{z_{\lambda}^{\prime \prime}}{z_{\lambda}}= & \mathcal{H}^{2}\left\{2-\epsilon+6 w-2 \epsilon\left[w+3\left(\frac{y_{\lambda}^{2}}{1-w}\right)\right]+12 y_{(1-\lambda)}^{2}+18\left(\frac{y_{\lambda}^{2}}{1-w}\right)\right. \\
& \left.+(\epsilon-3) x^{2}\left[1+2 w+6\left(\frac{y_{\lambda}^{2}}{1-w}\right)\right]+2 \frac{w^{\prime}}{\mathcal{H}}+\frac{6}{\mathcal{H}}\left(\frac{y_{\lambda}^{2}}{1-w}\right)^{\prime}\right\} \\
\aleph_{\lambda}= & \sqrt{6} \mathcal{H}^{2} \sqrt{2 \epsilon\left(1-x^{2}\right)}\left[\frac{1}{\mathcal{H}}\left(\frac{y_{\lambda}}{1-w}\right)^{\prime}+\frac{1}{2}(6-\epsilon-\delta)\left(\frac{y_{\lambda}}{1-w}\right)\right], \\
\beth= & 6 \mathcal{H}^{2}\left[\frac{1}{\mathcal{H}}\left(\frac{y_{+} y_{\times}}{1-w}\right)^{\prime}+6\left(3-\epsilon+(3-\epsilon) x^{2}\right)\left(\frac{y_{+} y_{\times}}{1-w}\right)\right] .
\end{aligned}
$$

\section{C.3 Caso Particular $\alpha=\pi / 2$}

Como enfatizado ao longo do texto, o caso $\alpha=\pi / 2$ é peculiar, pois corresponde ao único modelo de Bianchi I que não apresenta direções ricocheteantes durante o período anisotrópico primordial. Visto que estes modelos gozam de uma simetria planar (convenientemente escolhida para ser o plano $x y$ ), o componente $\sigma_{\mathrm{T} \times}$ do tensor de cisalhamento nestes casos é identicamente nulo para qualquer escolha dos ângulos de Euler da forma $(\alpha=0, \beta \neq 0, \gamma \neq 0)$, o que corresponde à uma simetria em torno do eixo $z$. Isso implica que a função $\aleph_{\times} \propto \sigma_{\times}$também se anula para todos os valores de $t$. O mesmo acontece com a função $\beth$, uma vez que essa se comporta como $\sigma_{+} \sigma_{\times}$e, pelo mesmo motivo, se anula. Para o caso particular (e apenas neste caso) $\alpha=\pi / 2$, temos que

$$
\aleph_{\times}=0 \quad \text { e } \quad \beth=0 .
$$

Isso simplifica bastante o cálculo da evolução das perturbações cosmológicas. Porém, como vimos, as previsões deste modelo não são genéricas.

A Fig. C.1 mostra que, nesse caso $\aleph_{+} / \mathcal{H}^{2} \rightarrow 0$ nos instantes iniciais de evolução, o que implica que os modos perturbativos nesse regime estão de fato desacoplados. As Figs. C.2 e C.3 apresentam a validade da aproximação WKB. Em particular, para este caso, podemos mostrar que em escalas inferiores ao raio de Hubble, temos

$$
\frac{z_{\mathrm{s}}^{\prime \prime}}{z_{\mathrm{s}}} \rightarrow \frac{k_{z}^{2}}{a_{z}^{2}}, \quad \frac{z_{\lambda}^{\prime \prime}}{z_{\lambda}} \rightarrow \frac{k_{z}^{2}}{a_{z}^{2}} .
$$

Além disso, checamos que existe apenas uma redefinição do tempo que leva a um novo conjunto de variáveis canônicas e que concordam com todas as conclusões encontradas em [23] (veja a discussão feita em §7.4).

Seguindo nosso procedimento de quantização, nós calculamos as predições para os espectros de potências (Figs. C.4 à C.5). Notemos que a simetria planar do modelo $\alpha=\pi / 2$ se faz óbvia em termos da projeção Mollweide, em comparação com o caso $\alpha=$ 
$\pi / 4$ apresentado na Fig. 7.10. Isso mostra que, em princípio, uma possível detecção de anisotropia primordial na RCF pode nos permitir reconstruir o modelo anisotrópico inflacionário exato. Como podemos ver, neste caso particular sempre existe um regime WKB, o que nos permite definir as condições iniciais da maneira usual. Seguindo o procedimento de quantização já detalhado, podemos calcular os espectros de potências para este modelo. Apresentamos portanto as figuras equivalentes às figuras da seção 7.7 .

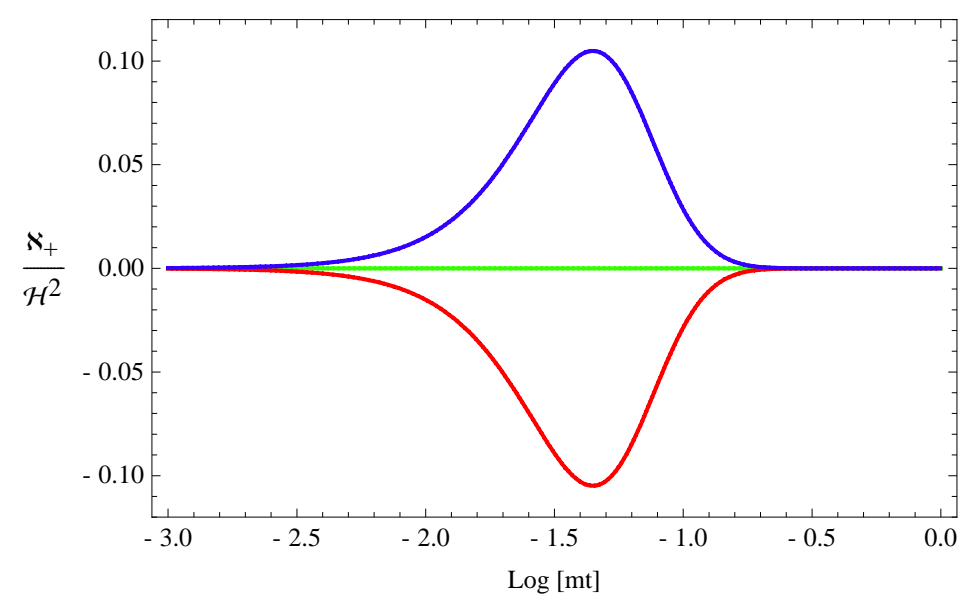

Figura C.1: Evolução da quantidade $\aleph_{+} / \mathcal{H}^{2}$ para os três modos ortogonais alinhados com os eixos $x, y$ e $z$ (representados por três cores diferentes).

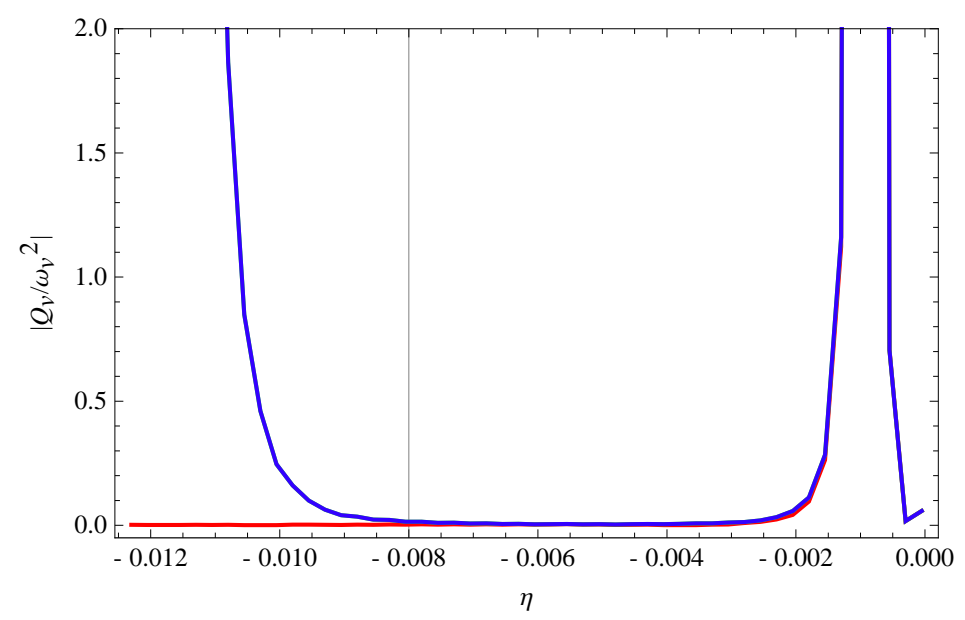

Figura C.2: Evolução da quantidade $\left|Q_{v}^{\mathrm{WKB}} / \omega_{v}^{2}\right|$ para três modos diferentes, cada qual alinhado com um dos três eixos ortogonais $x, y$ e $z$ e com o mesmo módulo $10 k_{\text {ref }}$ ao fim da inflação. 

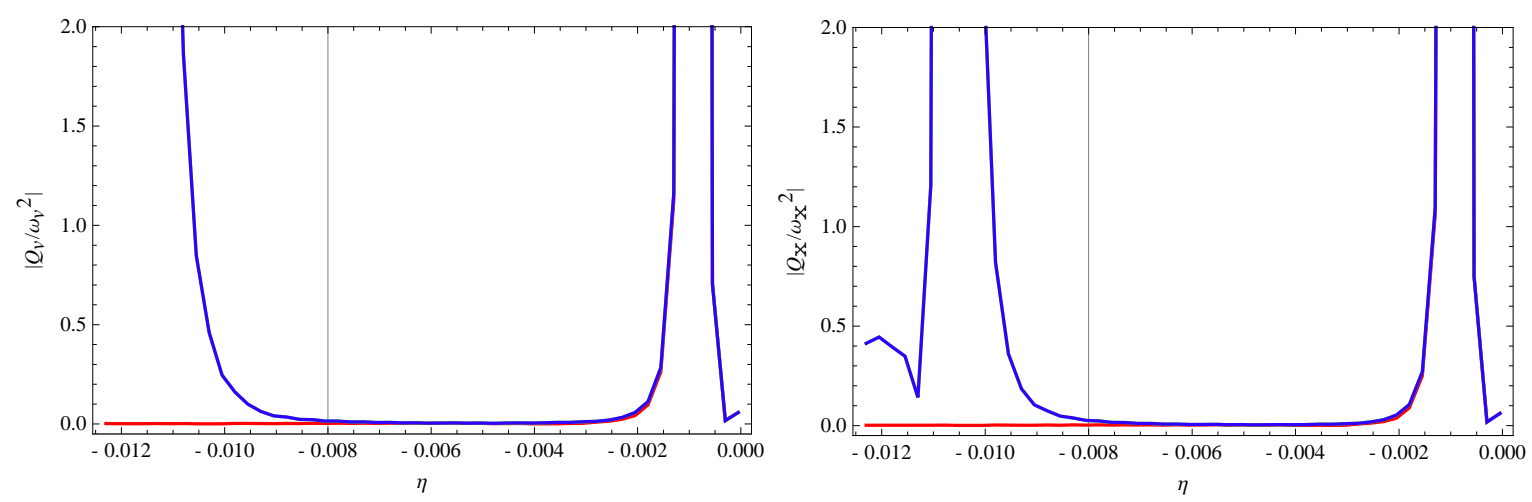

Figura C.3: Evolução das quantidades $\left|Q_{\lambda}^{\mathrm{WKB}} / \omega_{\lambda}^{2}\right|$ para $\lambda=+$ (esquerda) e $\lambda=\times$ (direita) para três modos ortogonais e com o mesmo módulo $10 k_{\text {ref }}$ ao fim da inflação.
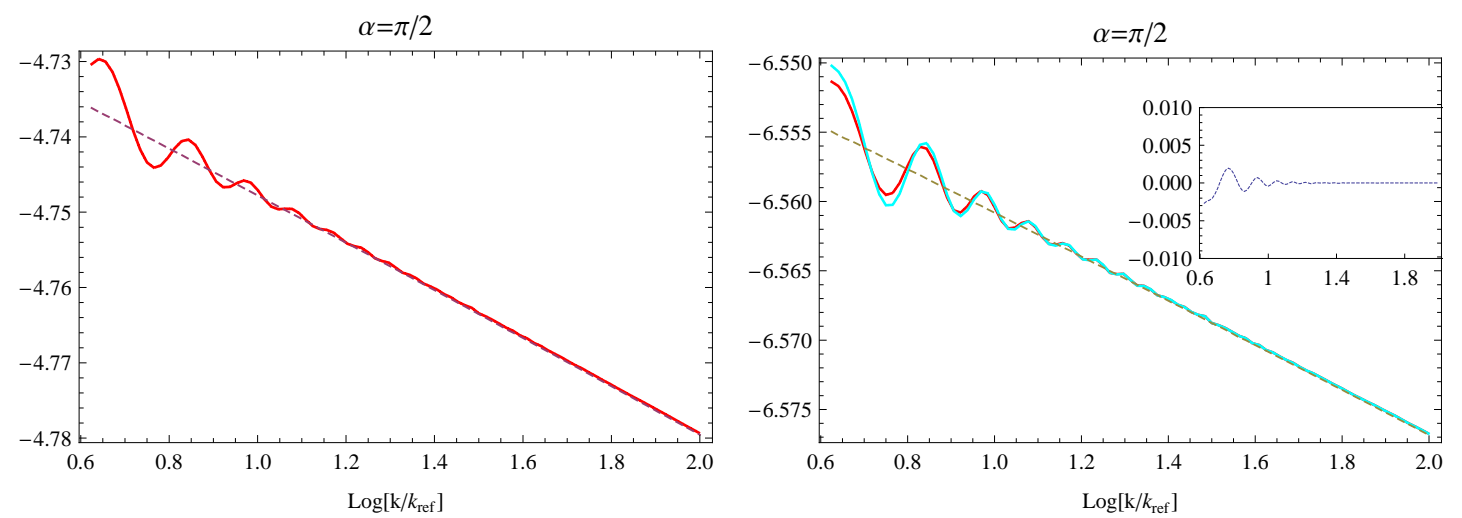

Figura C.4: Evolução das funções $f_{R}(k)$ (esquerda) e $f_{\lambda}(k)$ (direita) em termos de $\log \left[k / k_{\text {ref }}\right]$. O caso isotrópico (FRW) está representado pela linha pontilhada.
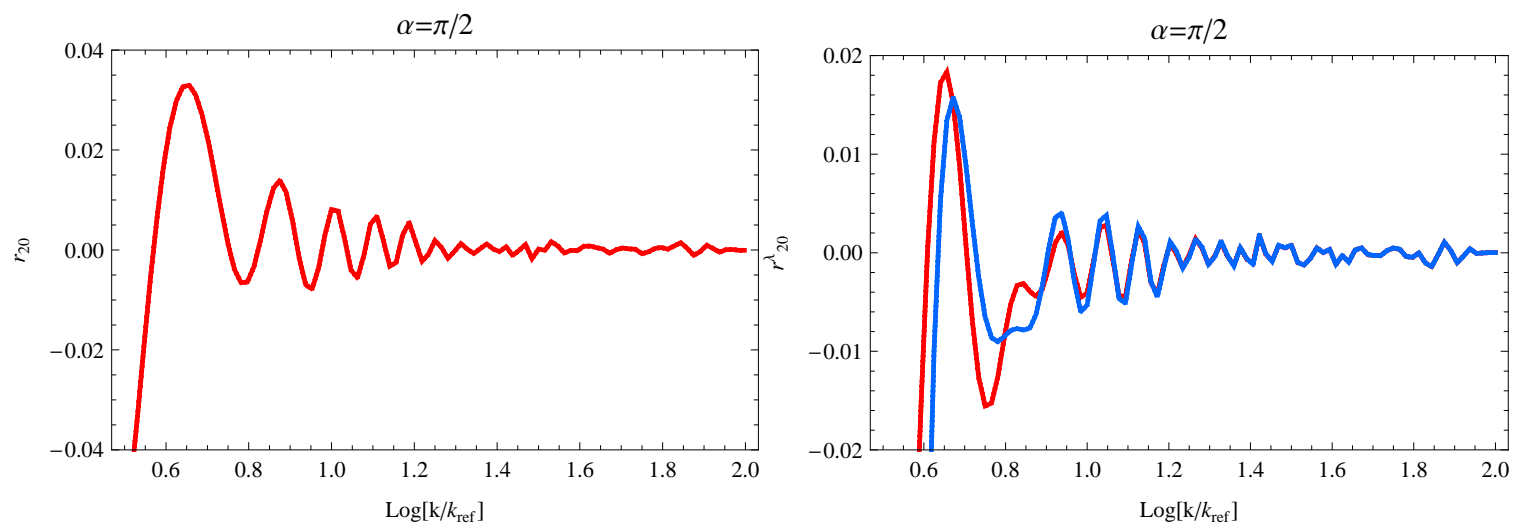

Figura C.5: Evolução dos coeficientes $r_{\ell m}(k)$ (esquerda) e $r_{\ell m}^{\lambda}(k)$ (direita) como função de $\log \left[k / k_{\text {ref }}\right]$ para os multipolos mais baixos $(\ell=2)$. O eixo $x$ foi arbitrariamente usado para a decomposição dos harmônicos esféricos. Como resultado das simetrias do espaço-tempo de fundo, apenas os coeficientes com $m=0$ são diferentes de zero. À direita apresentamos as polarizações + (curva vermelha) e $\times$ (curva azul). 

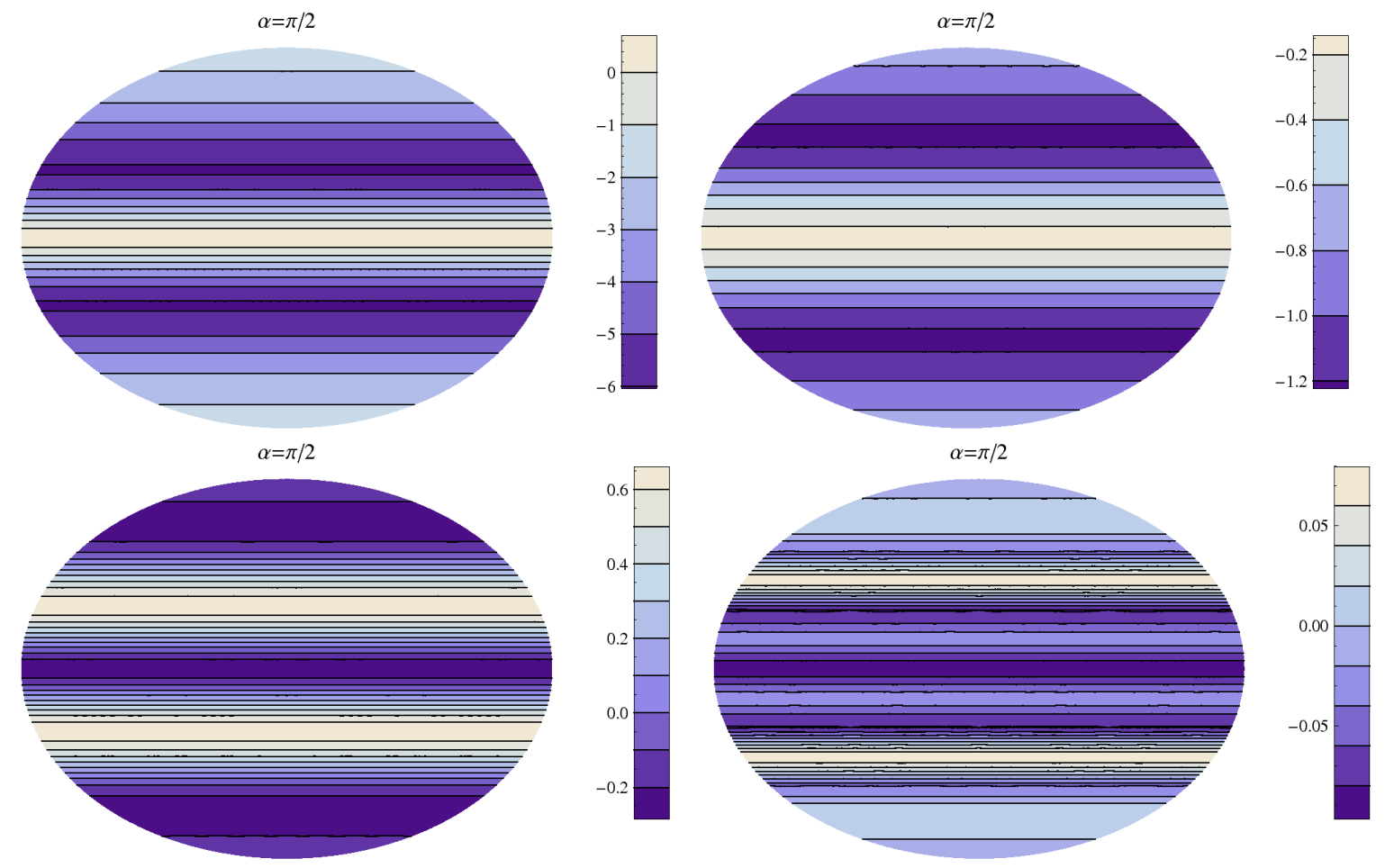

Figura C.6: Projeção Mollweide da razão entre $\mathcal{P}_{\mathcal{R}}(\mathbf{k})$ e seu valor isotrópico expressa em porcentagem para os valores $\log \left[k / k_{\text {ref }}\right]=1 / 2,3 / 4,1,3 / 2$ da esquerda para a direita e de cima para baixo. 